\title{
High throughput drug discovery in $S$. cerevisiae: The characterisation of FC-592 and FC-888
}

\author{
LIAM D. P. SAMPSON
}

2012

A thesis submitted to Victoria University of Wellington in part fulfilment of the requirements for the degree of Masters of Science in Biotechnology

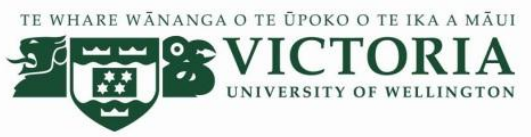

School of Biological Sciences

Victoria University of Wellington, New Zealand 
"The most beautiful thing we can experience is the mysterious. It is the source of all true art and all science. He to whom this emotion is a stranger, who can no longer pause to wonder and stand rapt in awe, is as good as dead: his eyes are closed." - Albert Einstein

"I haven't failed...I've found ten thousand ways that don't work." - Thomas Edison

"You find a gem in this \#*\&^@!" - David Maass 


\section{Abstract}

The discovery and characterisation of novel small molecule drug candidates is a medical priority. Recent advances in synthetic organic chemistry allow the de novo production of diversity oriented synthetic compound libraries and synthetic modification of natural products to provide candidate compounds for screening as potential therapeutics, bioactive agents or genetic probes. Small drugs function through interaction with complex genetic networks and pathways. However, it is difficult to characterise these interactions on a genome wide level to achieve understanding of drug mechanism. Here, discovery based approaches are utilised to achieve system wide parsing of biological mechanism, in an attempt to characterise the action of novel synthetic compounds and natural product derivatives.

Chemical genomic analysis allows for such understanding by examining growth profiles of a genomic deletion library of Saccharomyces cerevisiae mutants in the presence of sub-inhibitory concentrations of drug. The gene targets of small molecule compounds can be identified by noting deletion strains which display increased sensitivity, indicating chemical interaction with the associated gene network. In addition, the development and characterisation of resistant mutants can be used to identify putative drug targets. In this strategy, characterisation of the mechanism of resistance gives insight into drug mode-of-action. This study develops a high throughput yeast inhibition assay to identify bioactive compounds from a synthetic organic compound library, and attempts to characterise mechanism of action by establishing a profile of each compound's interaction with these gene networks; and mapping a resistance mutation to provide evidence of inhibitory mechanism. Two candidate compounds are identified, FC-592 and FC-888.

FC-592 displayed cytostatic inhibition. Further, yeast tag microarray homozygous profiling (HOP), chemical structure analysis, and cell-cycle analysis via flow cytometry for this compound provided evidence for a mechanism of poor specificity that targets glycoprotein biosynthesis and the secretory (Sec) pathway, as well as the cell-division cycle (CDC) pathway. Attempts to characterise a mutant resistant to this compound via synthetic genetic array mapping were unsuccessful when the resistance mutation proved to mediate a slow growth phenotype, abrogating the Synthetic Genetic Array Mapping approach utilised. Pending further analysis, it is suggested that this compound could have a role as a genetic probe in future exploration of the Sec and CDC pathways.

Chemical structure analysis and a non-specific HOP screen chemigenomic profile suggested that FC888 is an alkylating agent with a broad affinity for cellular nucleophiles. The compound demonstrates cytotoxic activity, and its efflux is not mediated by the pleiotropic drug resistance (PDR) network. It is suggested that the compound could find utility as a probe dissecting processes related to cellular defence against non-DNA specific alkylation. 


\section{Acknowledgements}

Well, to all of you who said I couldn't get it done... Actually, no-one said I couldn't get it done. I have been truly blessed to be surrounded by people who believed in me and gave me their unwavering support as I undertook the most significant academic task of my 25 years on this earth. There is no way that I would be here without you.

To Prof. Paul Atkinson of the Victoria University Chemical Genetics lab, thank you. You were an amazing supervisor who went above and beyond the call of duty when you took over my project half way through. For the jokes and the limericks and the conversations on liberal politics, thank you. May you always find the nine-letter word.

To Dr. David Bellows, who took a chance on a clueless kid from Rongotai, thank you. Your entertaining and informative lectures in undergrad are what brought me to Chem Gen in the first place. May your wine cellar always be full.

To Dr. Richard Furneaux, who made this project possible, thank you. To Rosemary Heathcott, Asoc. Prof. Paul Teesdale-Spittle, Dr. James Matthews, Dr. David Maass, Peter Bircham and Dr. Arun Kanakkanthara, thank you. This project would have been impossible without your help and advice experimentally. I will be forever grateful.

To Namal 'Usher' Coorey, who made the PDR strains I made ruthless use of, cheers bro. The little suckers probably hate you for taking away their drug resistance.

To the Chem Gen team, thank you. To Bede 'the fastest mullet in the west' Busby, Peter 'scrawniest guy in his family' Bircham, Daz 'Sasquatch' Jones, Katie 'I'm so disappointed in you' Zeier, Namal 'hungry hungry hippo' Coorey, cheers y'all. You and the Chem Gen family stopped me from going insane over the last two years, whilst frequently contributing to my delinquency. You will always have a friend.

To the people who have inspired me over the years to become the man I am, thank you. To Suze Randall and Dinesh Bindhi and Dave Reynolds and Phil Sparks, you told a wayward young kid he could do anything, and he believed you. To Brent Sarten, thanks skipper. You are a role model. To the Dead Ants, who yielded great memories on and off the rugby paddock, my battered body thanks you.

To my family, thank you is not enough. To Erin, thanks for the fun times and the alcohol poisoning. We should have a beer sometime. To KT, words can never express. To Mum, my greatest supporter, who always believed in me. And to Dad, who showed me what it means to be a man. None of this would have been possible without you.

And I don't just mean this thesis. 


\section{Contents}

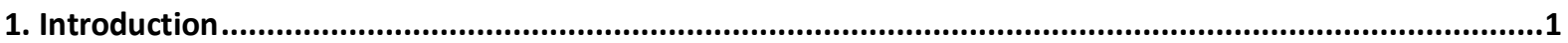

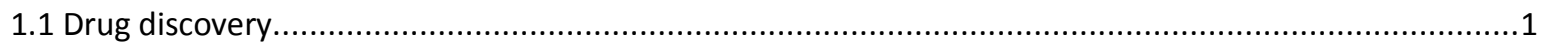

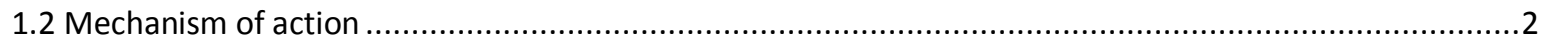

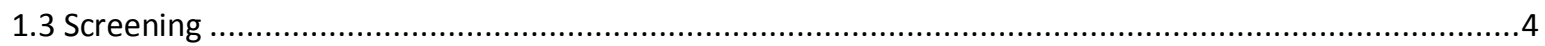

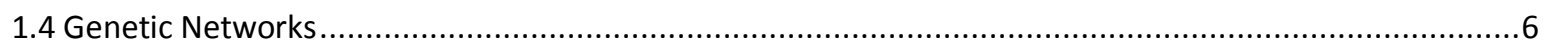

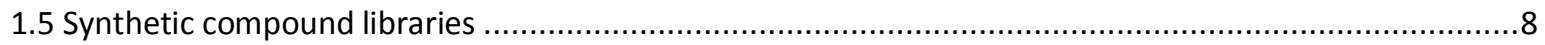

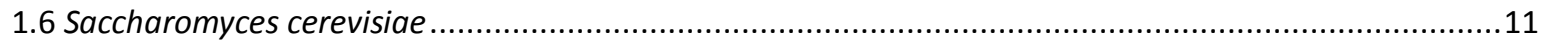

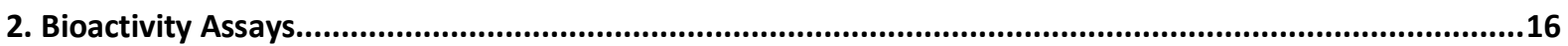

2.1. Introduction

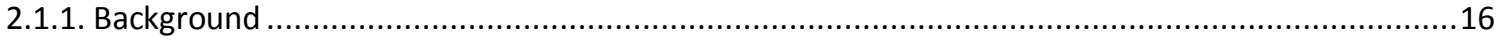

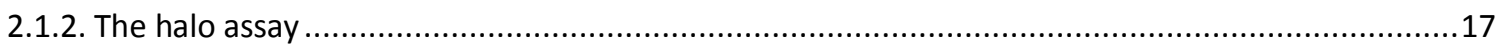

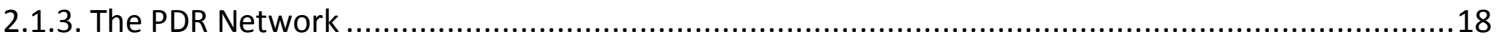

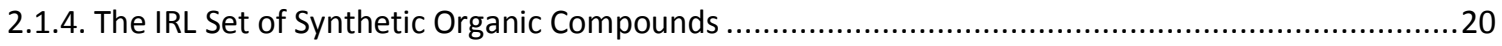

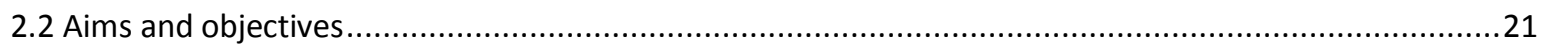

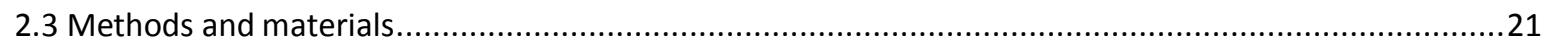

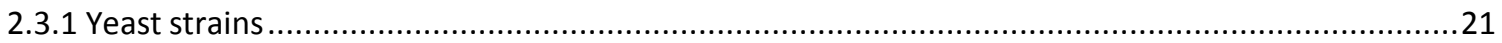

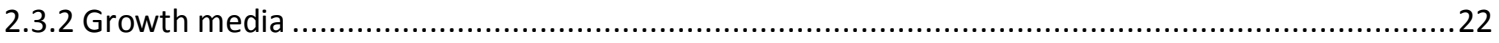

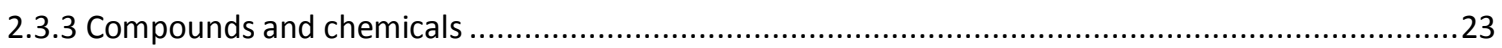

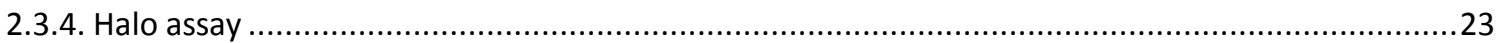

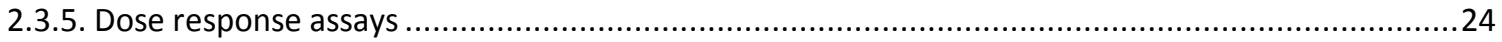

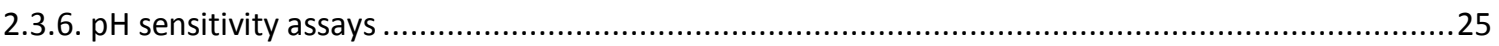

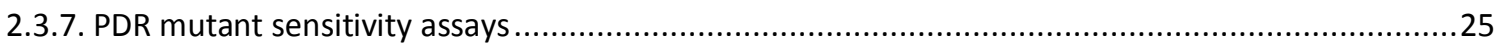

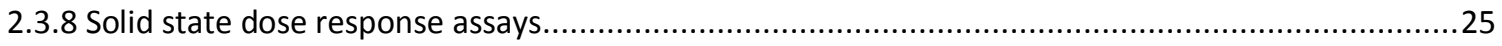

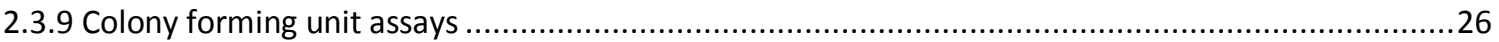

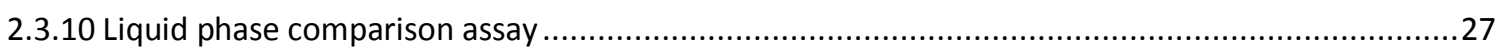

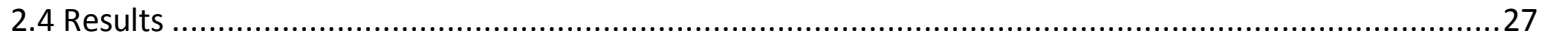

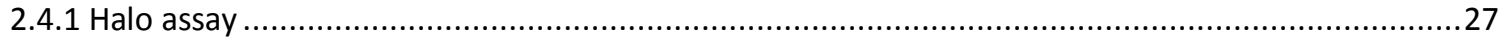




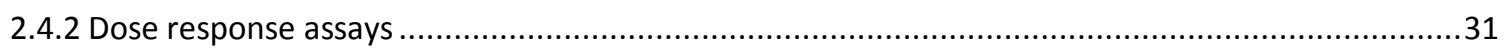

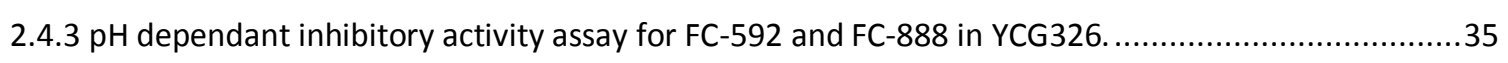

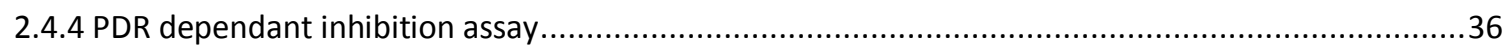

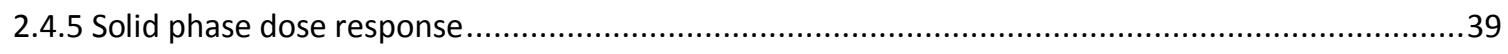

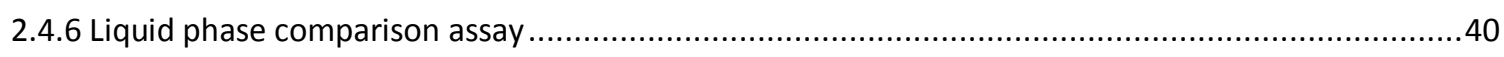

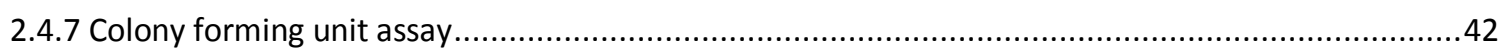

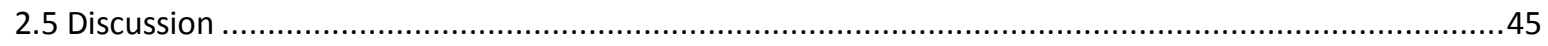

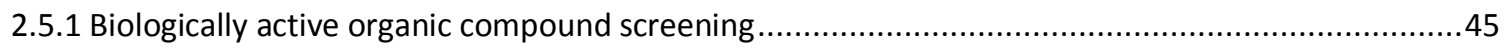

2.5.2 The halo assay as an inhibition screen in yeast ............................................................... 46

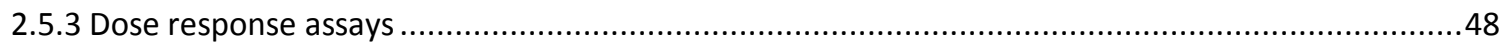

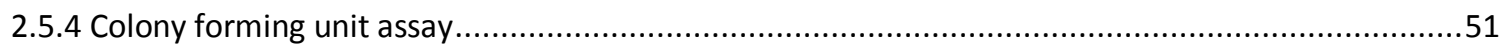

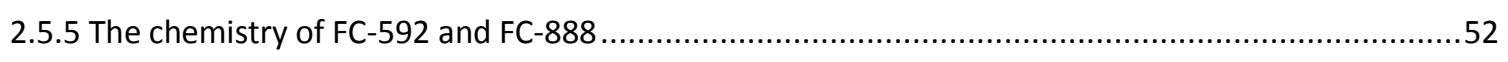

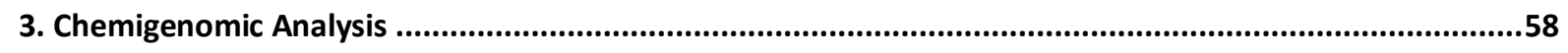

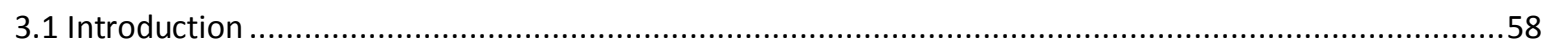

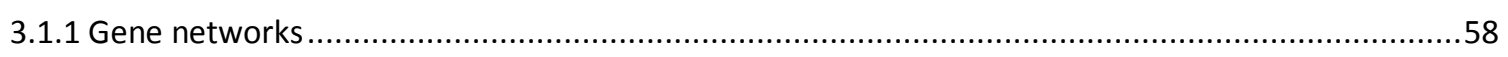

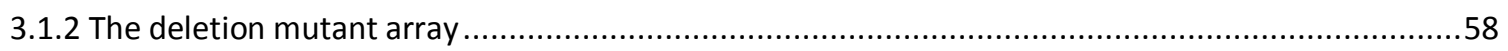

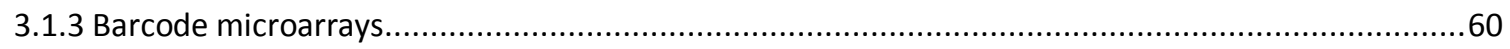

3.1.4 Glycosylation biosynthetic processes and the unfolded protein response................................61

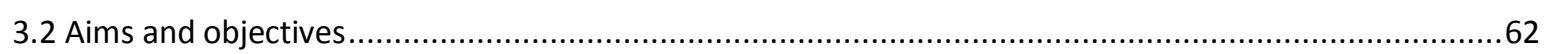

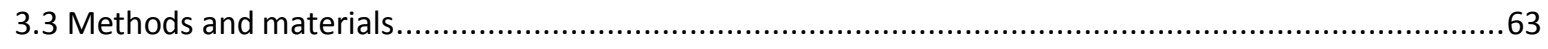

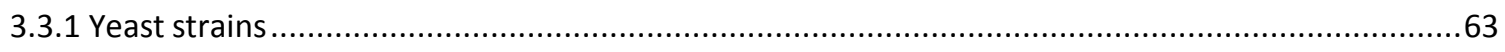

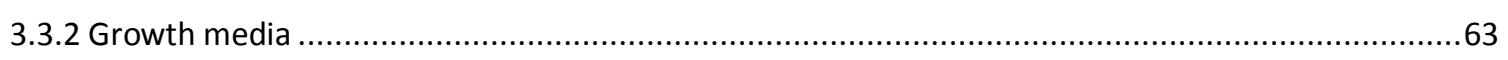

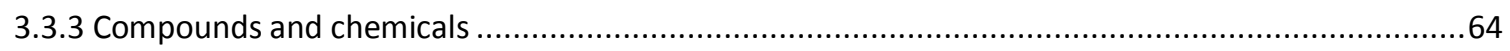

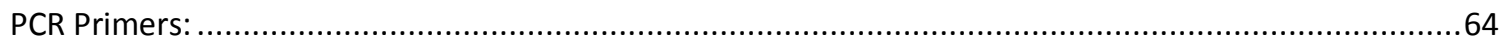

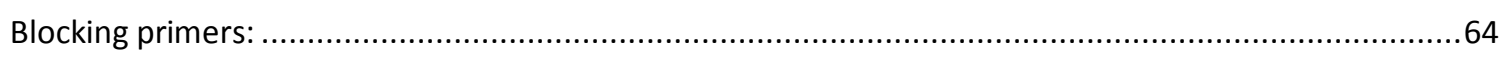

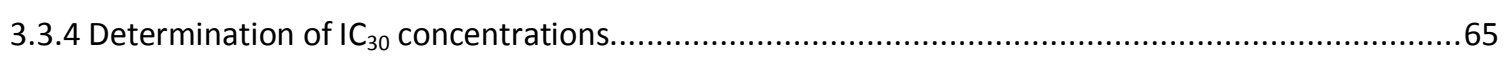

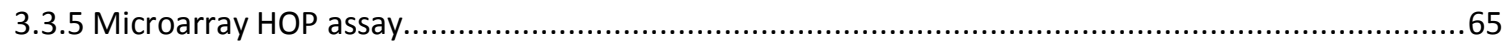


DNA purification

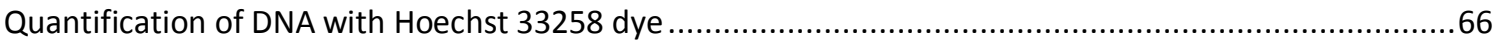

PCR of UP and DN tags with Cy3 and Cy5 dye labelled primers.................................................67

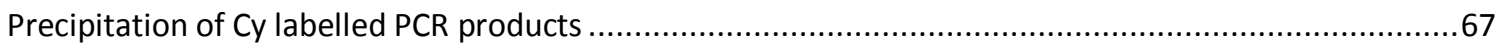

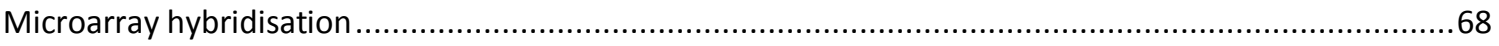

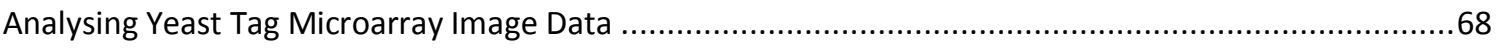

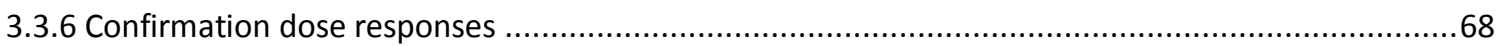

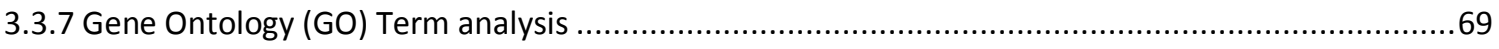

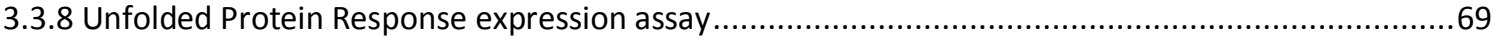

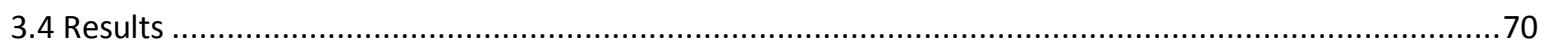

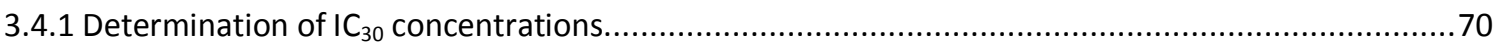

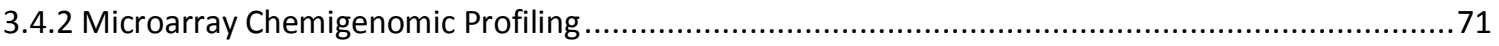

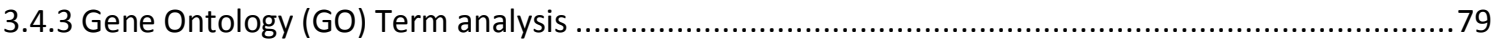

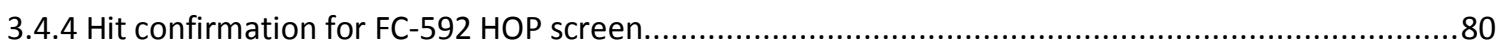

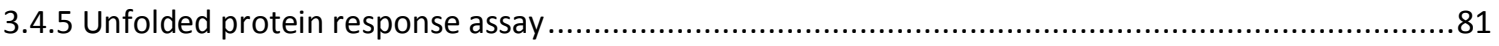

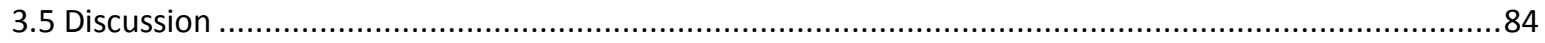

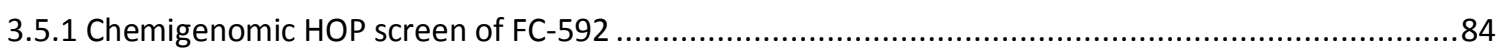

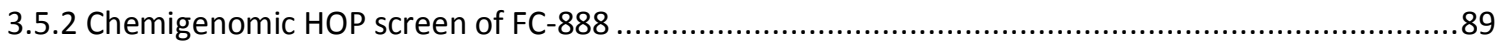

3.5.3 Assessment of yeast tag microarray HOP screen as an experimental method .............................91

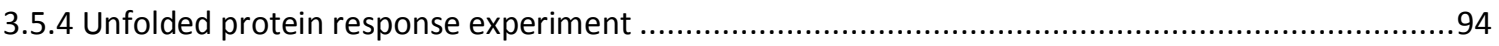

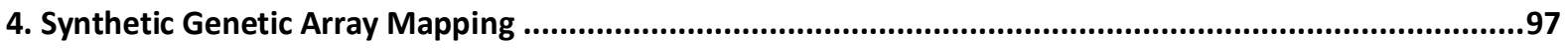

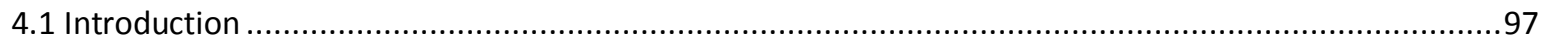

4.1.1 Resistance mutation as a target identification strategy ...................................................97

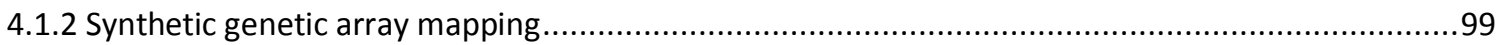

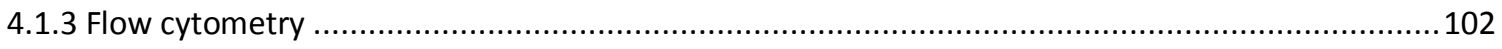

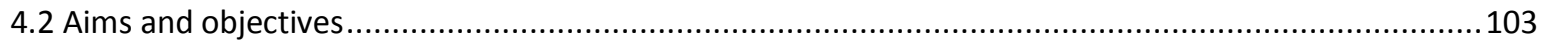

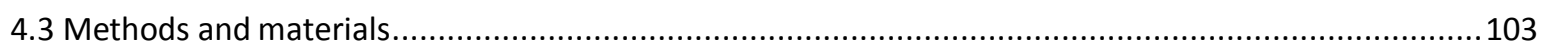

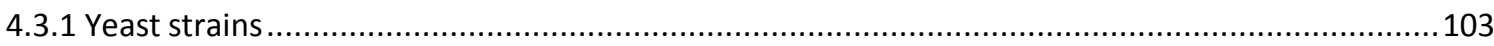




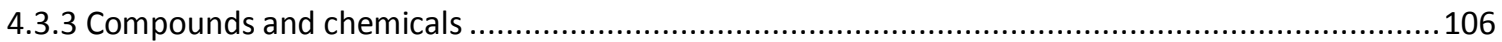

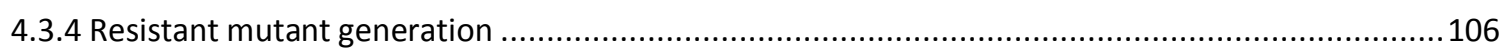

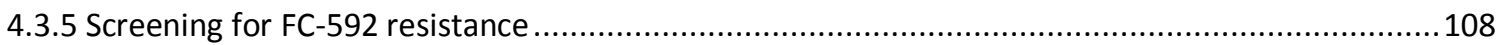

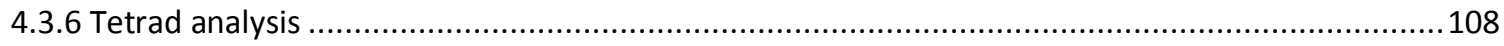

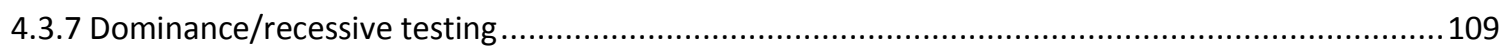

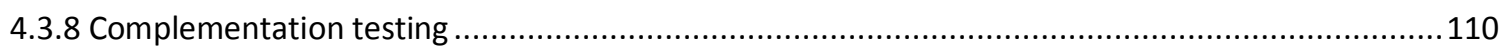

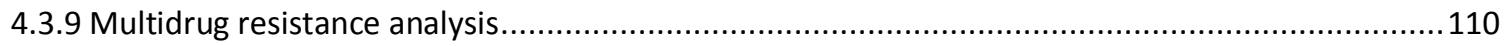

4.3.10 Growth rate comparison of resistant mutants........................................................ 111

4.3.11 Synthetic genetic array mapping - proof of concept.................................................... 111

4.3.12 Synthetic genetic array mapping - FC-592 resistant mutant .......................................... 112

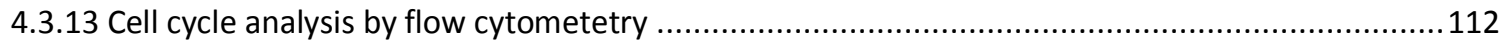

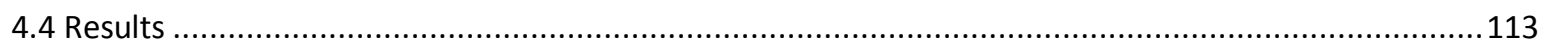

4.4.1 Resistant mutant generation .................................................................................... 113

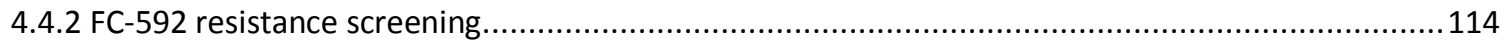

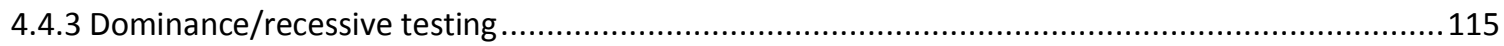

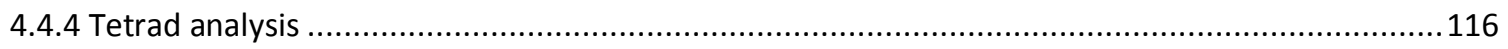

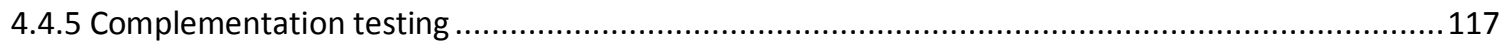

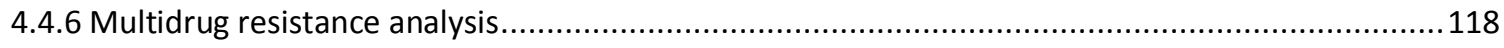

4.4.7 Growth rate of resistant mutants............................................................................ 121

4.4.7 Synthetic genetic array mapping (SGAM) - proof of concept.................................................. 122

4.4.8 YCG434 FC-592 resistant mutant - Synthetic genetic array mapping ...................................124

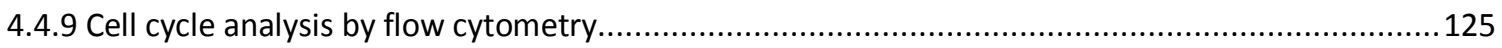

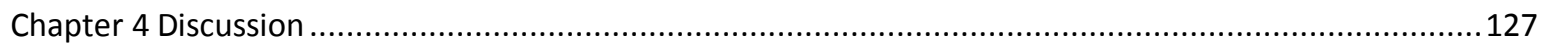

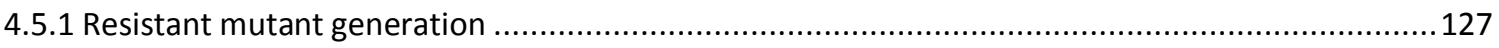

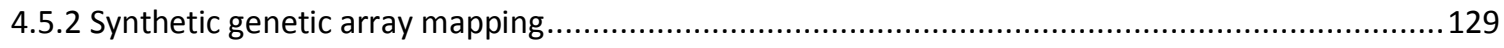

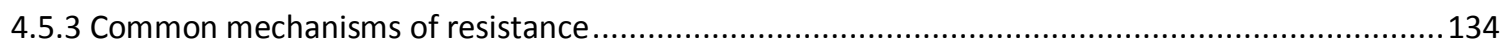

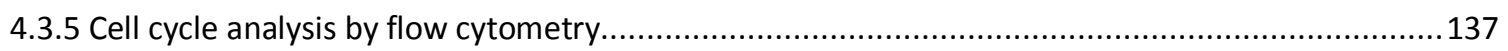


5.1.1 Mode of action for FC-592 and FC-888.

5.1.2 Assessment of FC-592 and FC-888 utility.

5.1.3 Assessment of experimental strategies and protocols.

5.1.1 Assessment of aims and objectives

6. Future Directions

6.1 Future directions in FC-592

6.1.1 Haploinsufficiency profiling assay in FC-592 .....

6.1.2 Genomic over-expression assay in FC-592

6.1.3 GFP-linked proteomics assay in FC-592

6.1.4 Secretory pathway screen in FC-592

6.1.5 Characterisation of FC-592 resistance mutations by sequencing.

6.1.6 Characterisation of FC-592 resistance mutations by plasmid rescue

6.1.7 Characterisation of FC-592 resistance mutations by liquid phase SGAM

6.1.8 Further investigation in cell-cycle analysis.......................................................... 153

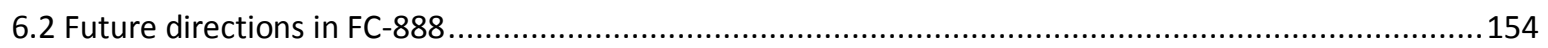

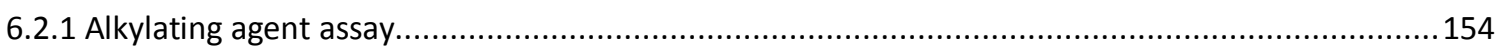

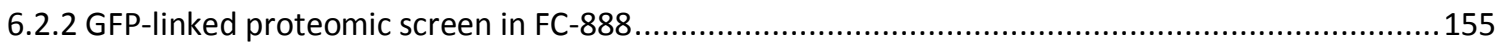

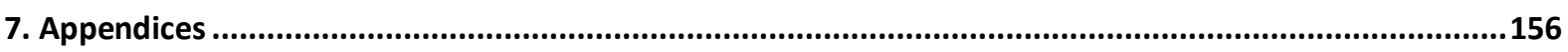

7.1.1 Appendix 1 - IRL compound library data .................................................................... 156

7.1.2 Appendix 1 - Plate layout data for dose response screen ............................................... 159

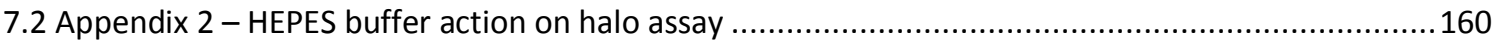

7.3 Appendix 3 - confirmation of PCR product for HOP screen ............................................... 161

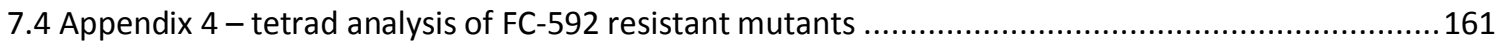

8. References 


\section{Tables and Figures}

Figure 1.1 An example of a genetic regulatory network $\quad 7$

Figure 1.2 The ADE2 assay identifies cells with successful plasmid expression 12

Figure 2.3 Distinctive 'halo' patterns 17

Figure 2.4 Regulatory transcription factors of the PDR network 18

Figure 2.5 Halo assay shows inhibitory activity in novel compounds 30

Table 2.1 Data on IRL compounds recording hits in halo assay 31

Figure 2.6 FC-592 32

Figure 2.7 FC-888 $\quad 32$

Figure 2.8 Dose responses for IRL set hits with YCG326 model 33

Table 2.2 17 MICs of the IRL compound library 34

Figure 2.9 pH Dependency in FC-592 35

Figure $2.10 \mathrm{pH}$ Dependency in FC-888 36

Figure 2.11 FC-592 dose response in PDR mutants 37

Figure 2.12 FC-888 dose response in PDR mutants $\quad 37$

Figure 2.13 Cycloheximide dose response in PDR mutants 38

Figure 2.14 Solid state dose responses reveal the sensitivity of YCG326 yeast cells to FC-592
and FC-888

Figure 2.15 Inhibitory compounds from the IRL compound library liquid inhibition assay 41

Figure 2.16 DMSO treated colony forming unit assay $\quad 42$

Figure 2.17 Cycloheximide treated control colony forming unit assay 42

Figure 2.18 Rapamycin treated control colony forming unit assay 43

Figure 2.19 FC-592 treated cells colony forming unit assay 43

Figure 2.20 FC-888 treated cells colony forming unit assay 43

Figure 2.21 Halo assay test plates $\quad 47$

Figure 2.22 Comparison of inhibition assay results 47

Figure 2.23 Lewis diagram anion formation $\quad 52$

Figure 2.24 Polygodial $\quad 54$

Figure 2.25 Nucleophilic substitution of bromine with a cellular nucleophile 55

Figure 2.26 Nucleophilic substitution of FC-888 is completed 55

Figure 3.1 Gene deletion strains are created 58

Figure 3.2 The DMA can be used to identify drug targets $\quad 60$

Figure 3.3 Parallel analysis of large pools of deletion mutants $\quad 60$

Figure 3.4 Narrow range dose response of FC-592 \& FC-888 70

Table 3.1 List of hypersensitive mutants derived from HOP screen of FC-592 treated cells $\quad 74$

Table 3.2 List of hypersensitive mutants derived from HOP screen of FC-888 treated cells 78

Figure 3.5 Genetic networks enriched for genes linked to FC-592 sensitivity 79

Figure 3.6 Confirmation screen for FC-592 HOP assay hits 81 
Table 3.3 Induction of UPR is not observed in FC-592 treated cells

Figure 3.7 YCG326 cells treated for $4 \mathrm{~h}$ with $8 \mathrm{mM}$ DTT

Figure 3.8 YCG326 cells treated for $4 \mathrm{~h}$ with $112 \mu \mathrm{M}$

Figure 3.9 Cluster analysis of FC-592 hits

Figure 3.10 Cluster analysis of FC-888 hits

Figure 4.1 Examples of SGA methodology 100

Figure 4.2 Synthetic genetic array mapping 100

Figure 4.3 Homologous recombination in SGAM 101

Figure 4.4 A linkage pattern is seen around the site of resistance in a SGAM experiment 101

Figure 4.5 Mutagenised cells treated with 3\% EMS for $1 \mathrm{~h} \quad 113$

Figure 4.6 Unmutagenised control population 113

Figure 4.7 Mutagenised cells screened on 90 $\mu \mathrm{M}$ FC-592 114

Figure 4.8 Mutagenised cells screened on 110 MM FC-592 114

Figure 4.9 Solid state dose response assay of resistant mutants 115

Figure 4.10 Spot assays on $150 \mu \mathrm{M}$ FC-592 116

Figure 4.11 Tetrad analysis of resistant mutants 116

Figure 4.12 Dose response assay showing complementation 117

Figure 4.13 Multidrug resistance analysis of the FC-592 resistant strains YCG433 and YCG434 121

Figure 4.14 Time series growth assay 122

Figure 4.15 A linkage disequilibrium pattern is observed on plate three of the SGAM 123

Figure 4.16 The genomic location of RPL28 124

Figure 4.17 Plate 7, YCG434 SGAM 124

Figure 4.18 Resistance phenotypes 125

Figure 4.19 Three replicates of FC-592 treated cells compared to untreated cells in a FACS

$\begin{array}{ll}\text { experiment } & 126\end{array}$

Figure 4.20 Growth phenotype colonies were few and far between $\quad 129$

Figure 4.21 Tetrads growing on SC media 133

Figure 4.22 Tetrad phenotyping on $150 \mu \mathrm{M}$ FC-592 133

Figure 4.23 Comparison of FC-592 treated cells 138

Figure 4.24 Mammalian component of the Cdk pathway regulating entry to mitosis 139

Table 7.1 Molecular data on compound library from IRL 156

Table 7.2 Plate layout for original dose response 159

Figure 7.1 Conditional test based on the halo assay 160

Figure 7.2 Confirmation of PCR product for HOP screen 161

Figure 7.3 Mat A selection of YCG434 meiotic progeny 161

Figure 7.4 Selection on $150 \mu$ M FC-592 media 162

Figure 7.5 Phenotyping of Tetrad \#9 162 


\section{List of Abbreviations}

\begin{tabular}{|c|c|}
\hline$A B C$ & ATP binding cassette \\
\hline ADME & Absorption, distribution, metabolism, excretion \\
\hline ATP & Adenosine triphosphate \\
\hline Bp & Base pair \\
\hline Can & Canavanine \\
\hline CDC & Cell-division cycle \\
\hline $\mathrm{CHX}$ & Cycloheximide \\
\hline $\mathrm{ddH}_{2} \mathrm{O}$ & Deionized distilled water \\
\hline DMA & Deletion mutant array \\
\hline DMSO & Dimethyl sulfoxide \\
\hline dNTP & Deoxyribonucleotide triphosphate \\
\hline DTT & Dithiothreitol \\
\hline EMS & Ethyl methyl sulfonate \\
\hline FACS & Fluorescence activated cell sorter \\
\hline G418 & Geneticin \\
\hline GFP & Green fluorescent protein \\
\hline HEPES & 4-(2-hydroxyethyl)-1-piperazineethanesulfonic acid \\
\hline HIP & Haploinsufficiency profiling \\
\hline HOP & Homozygous profiling \\
\hline HPLC & High-performance liquid chromatography \\
\hline IRL & Industrial Research Limited \\
\hline KanR & Kanamycin resistance cassette \\
\hline MDR & Multi-drug resistance \\
\hline MIPS & Munich Information Center for Protein Sequences \\
\hline MOBY-ORF & Molecular-barcoded yeast open reading frame library \\
\hline MOPS & 3-(N-morpholino)propanesulfonic acid \\
\hline Nat & Nourseothricin \\
\hline NatR & Nourseothricin resistance cassette \\
\hline OD & Optical density \\
\hline ORF & Open reading frame \\
\hline PCR & Polymerase chain reaction \\
\hline PDR & Pleiotropic drug resistance \\
\hline PEG & Polyethylene glycol \\
\hline QTL & Quantitative trait loci \\
\hline Rap & Rapamycin \\
\hline
\end{tabular}


SC

SD

SDS-PAGE

Sec

SGA

SGAM

$\mathrm{SL}$

SNP

SS

Thia

WT

YPD

$\triangle$ PDR
Synthetic complete

Synthetic drop-out

Sodium dodecyl sulfate polyacrylamide gel electrophoresis Secretory pathway

Synthetic genetic array

Synthetic genetic array mapping

Synthetic lethal

Single nucleotide polymorphism

Synthetic sick

Thialysine

Wild type

Yeast peptone dextrose

Attenuated pleiotropic drug resistance 


\section{Introduction}

\subsection{Drug discovery}

Drug discovery is the process by which compounds are discovered or designed, identified and characterised in order to perform as therapeutic agents or for some other biologically useful task. A multidiscipline science, drug discovery combines expertise in the diverse fields of medicine, molecular biology, pharmacology and chemistry. In the past many drugs have been discovered either by identifying the active ingredient from traditional remedies (Fabricant and Farnsworth, 2001) or by serendipitous discovery (Drews, 2000). However, more modern understanding in chemistry, genetics, physiology and biotechnology allow a much more purposeful approach, accelerating the drug discovery pipeline (Warrington, 2003).

This is necessary because there are many thousands of diseases that affect the human body, reducing life spans and causing suffering all over the world. Causes include viruses, pathogenic micro-organisms such as bacteria and fungi, cancers and the breakdown of normal biological function. The search is on going to identify novel molecular entities (NMEs) that could be of potential benefit in combating these illnesses and reducing patient mortality and morbidity.

In the modern world, this is most often achieved through high throughput screening techniques which allow a large array of potential drugs to be assayed in a time and cost effective manner. Advances in genetics, computer modelling and pharmacology have meant that target based assays that begin with picking out a medically relevant drug target (Gibbs, 2000), and rational drug design (Thiell et al. 2003) are possible. Improvements on legacy techniques are required because drug discovery is still a lengthy, "expensive, difficult, and inefficient process" with low rate of new therapeutic discovery (Anson et al. 2009). The funding limitations faced by research facilities mean that cost is a critical factor, with the average research and development cost associated with each NME approaching US\$1.8b over a time period of 13.5 years (Paul et al. 2010). 
Finding new drugs through traditional means is becoming increasingly difficult as obvious drug targets have been thoroughly exploited. This means there few if any single major gene targets left in the art and design of new drugs. In many cases, the drugs that we rely on today (so called trade drugs) are suboptimal in their function. Current classes of antibiotic drugs are being rendered useless by newly evolving resistant microbes (Arrias and Murray, 2009). The problem is made worse because no new antibiotic compound structures have discovered since the mid 1960's (Payne, 2008). Microtubule stabilising anti-cancer drugs such as Paclitaxel, the mainstay of many current anti-cancer regimes, cause significant side effects such as bleeding, and have poor specificity for target (cancerous) cells (Goodman and Walsh, 2001). Of course, for many diseases, such as HIV, there is no current effective drug cure. These data indicate the continuing need for new and improved NMEs to treat the pathology of the $21^{\text {st }}$ century.

Areas in which NMEs could improve on the current pharmacopoeia include the efficacy against many diseases; safety and side effects; and factors such as absorption, distribution, metabolism, and excretion or ADME (Balani et al. 2005). In order to achieve advances in these areas it is vital to determine the mechanism of action of a biologically active compound.

\subsection{Mechanism of action}

The mechanism of action (MOA) refers to the specific biochemical interaction through which a drug substance produces its pharmacological effect. This is very important for selecting compounds with good target selectivity, a critical component of most therapeutic strategies. Understanding at the biochemical level how a given NME is achieving its effect also allows for the potential to optimise delivery, adjust dosage, combine with other chemical agents or even make synthetic modifications to the molecule itself to improve therapeutic outcomes (Sliwkowski et al. 1999). From a pecuniary perspective, MOA understanding is vital to patent applications and the intellectual property protection that allows a NME to progress to market (United States Court of Appeals for the Federal Circuit, 2008-1248). 
A critical part of early phase drug development is the identification of off-target drug effects, known colloquially as side effects. NMEs could potentially affect multiple targets, or a single target could itself affect multiple pathways and processes. Statin drugs, used to lower cholesterol levels by competitive inhibition of the enzyme HMG-CoA reductase (Lewington et al. 2007) also cause a reduction in systemic levels of ubiquinone (Co-enzyme Q10) due to its shared biosynthetic pathway with cholesterol (Kishi et al. 1977). This is associated with muscular myopathy (Marcoff and Thompson, 2007). Early identification of such off target effects allows for potential amelioration: reworking of the compound in chemical synthesis or some other strategy, or else discarding the compound before resources are spent developing it further.

In the worst case a widely used drug could cause severe pathological effects across a population before such side effects were identified, such as in the case of the anti-nausea and chemotherapeutic drug thalidomide. Widely prescribed for pregnancy associated morning-sickness, thalidomide was withdrawn in 1961 after being found to cause severe birth defects (Silverman, 2002). Interest in thalidomide relates to its multiple targets, including inhibition of the production of interleukin-6 (IL-6), activation of apoptotic pathways through caspase 8-mediated cell death (Anderson, 2005) and inhibition of tumour necrosis factor-alpha (TNFa)(Burkholz, 1997) providing a range beneficial effects including suppression of multiple myeloma and reduction in leprosy associated necrosis as well as its analgesic and antiemetic properties.

However, thalidomide's glutarimide moiety intercalates in guanine-cytosine rich regions of DNA (Koch and Czejka, 1986; Huang and McBride, 1997). In utero, such effects inhibit expression of IGF-I and FGF-2, genes which stimulate angiogenesis of developing limbs, ears and eyes (Stephens et al. 2000). If such off target effects could have been identified at the laboratory stage, much damage in the original use of this drug would have been avoided.

It is also important to note that not all such off target effects are harmful. Drugs with promiscuous activities have proven their worth in combating multiple diseases. The $\gamma$-aminobutyric acid analogue 
Gabapentin, approved for treatment of seizures and postherpetic neuralgia in adults, has sideeffects which are useful in treating neurological conditions such as bipolar disorder, essential tremor, hot flashes, migraine prophylaxis, neuropathic pain syndromes, phantom limb syndrome, and restless leg syndrome (Vedula et al. 2009). In these cases a validated characterisation of an NME's genome wide mechanism of action could lead to further applications than a limited target based screen would be able to.

\subsection{Screening}

NME screening strategies are increasingly target based (Schreiber, 2000; Winzeler et al. 1999). Target-based drug discovery begins with the identification of the function of a potential therapeutic drug target and understanding its role in the disease process. In this manner, it can be thought of as a 'reverse' type screen, where the answer is known (the drug target) and the question must be found (the drug). However, the adoption of target based strategies has not resulted in increased drug discovery, and is in fact correlated with a decline in NMEs adopted for clinical trial (Sams-Dodd, 2005).

There are several issues associated with this approach. Target based discovery requires a validated drug target to pursue, and the process of target validation is complex and associated with a high degree of uncertainty. In addition, many pathologies are too complex to be targeted by a single gene or pathway based assay (Sams-Dodd, 2005, Sams-Dodd, 2006). Further, a drug discovery assay limited to screening for a specific target effect risks overlooking other potential functionalities of candidate compounds - a serious problem if such compounds are unique or rare.

An alternative approach to assaying NMEs with biological activity is a mechanism based, 'forward' screen. This strategy seeks to characterise the mechanism of action of a known bioactive compound and takes advantage of the ease of screening for broad based biological activity via inhibition assays. This allows a far greater reach in the mechanism space explored: it is possible to find drugs affecting a greater range of targets than those for which there are adequate models. 
A 'forward' screening approach takes advantage of in vivo assay design. By definition, the entire biological system must be present to determine the drug target. This prevents the use of biochemical based in vitro designs, but allows for characterisation against a more realistic model, an important consideration during drug development (Sams-Dodd, 2006).

Comparatively recent advances in molecular biology have allowed screening in this manner. The characterisation of the genome and gene manipulation techniques have given rise to a set of tools and a level of understanding which allow for sophisticated probes into a molecule's function within the cell. Conclusions can be drawn from an array of data according to our understanding of the rules of genetics and biological function. In this way, like piecing together a puzzle, powerful determinations can be made that would not be possible via simple biochemical routes. Assays incorporating genomics and the construction of gene deletion libraries are among those techniques that can be used to assess drug mechanism.

At this point it should be noted that simply finding new therapeutics is not the only possible goal of this type of research. Many small molecule inhibitors (SMIs) such as cycloheximide, which inhibits protein synthesis (Schneider-Poetch, 2010) can be used as functional probes to dissect gene mechanism, protein function, genetic pathways and other cellular processes. Research encompassing the 'forward' approach could also reveal compounds that act on certain pathways, allowing them to be used as probes in this manner.

Many such probes are useful for activating or inactivating certain protein functions, providing tools to differentiate gene function in healthy and diseased cells (Strausberg and Schreiber, 2003). The US National Cancer institute has taken this approach with their "Cancer Drug Discovery: Diversity Generation and Smart Assays" program (Strausberg and Schreiber, 2003), of which a critical component is the construction of a diverse array of SMI probes.

In designing small molecule bioactivity screens, it is crucial to maximise the utility of the generally small amounts of compound available. In a research setting, obtaining additional quantities of novel 
compounds is often difficult, so minimising usage in assay design is essential. Several strategies for reducing compound usage are discussed in further chapters.

\subsection{Genetic Networks}

The central dogma of molecular biology holds that a gene produces an RNA transcript which produces a protein which produces biological work. As Francis Crick (1970), the original framer of this concept himself states, the overall genetic control of a cell is far more complicated. Drugs typically work through interacting with genes and gene products to have an effect on biological processes. However, genes typically do not work in isolation, but work with each other in complex pathways and networks.

A gene network or genetic regulatory network (GRN) is a collection of genes, which may be located throughout the genome, which interact with each other through their RNA and protein products, along with other cellular components to control cellular processes by governing the rates at which genes in the network are transcribed into mRNA (Davidson and Levin, 2005). Protein products can include structural elements and enzymes, amongst others which do work within the cell, as well as transcription factors which increase or decrease the expression of other genes. In this way it can be seen that a gene network is a complex web of genes which can perform multifaceted tasks via its regulatory mechanisms. 


\section{HFE}

\section{A GENE REGULATORY NETWORK}

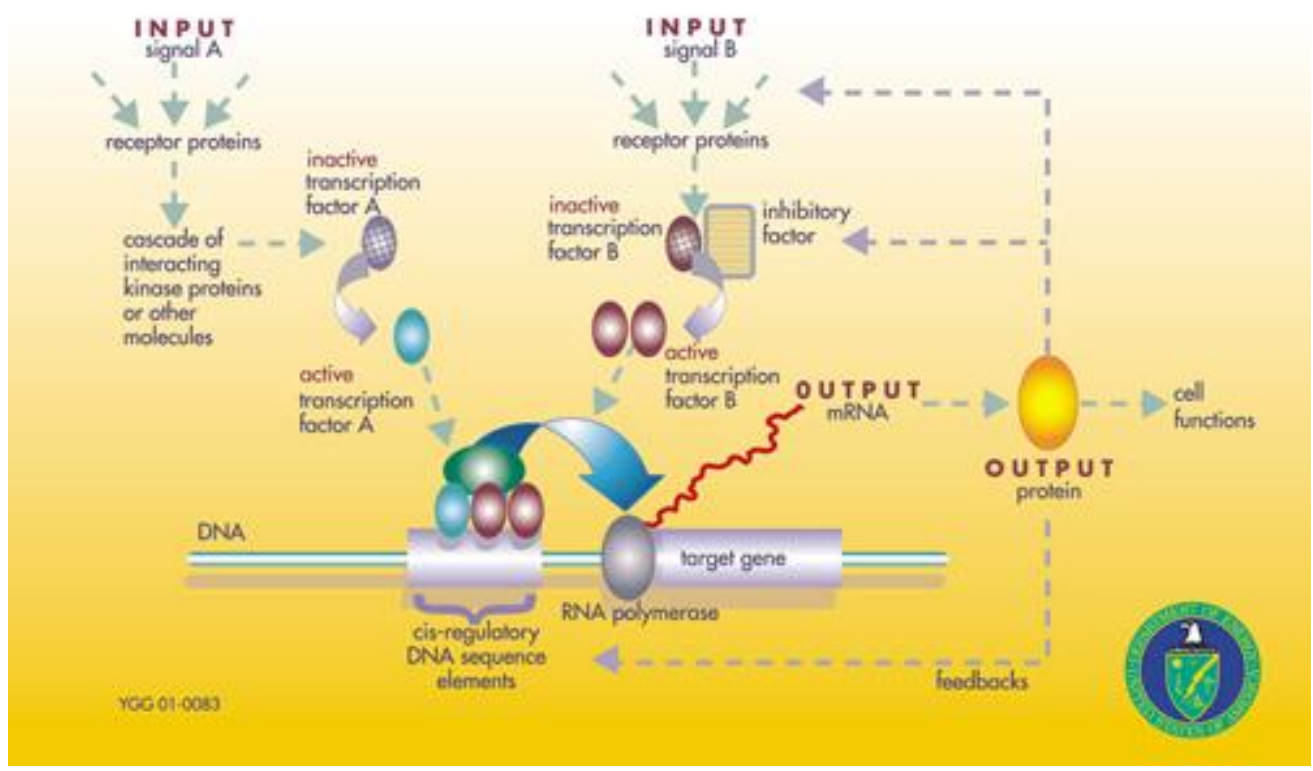

Figure 1.1 An example of a genetic regulatory network. Receptor proteins respond to stimulus in the form of input signals, for example a certain cellular toxin, and activate a promoter for another gene which produces a protein to respond to the stimulus. This protein might activate further genes and so on, until a complex response is produced to a specific set of conditions. Source: United States Department of Energy. Public Domain.

A further characteristic of genetic networks is their 'buffered' nature. A series of redundant genes and pathways means that many regulatory and developmental systems produce phenotypes that are robust to environmental and genetic variation (Levy and Siegel, 2008). Genes and gene network nodes which contribute to this robustness are known as phenotypic capacitors and help an organism respond to varied stresses by covering up altered gene expression caused by genetic mutation or environmental stress. From an evolutionary standpoint, this provides a mechanism for genetic polymorphisms to accumulate without significantly ablating cell function. The prime example of this phenomenon is Hsp90, a molecular chaperone that targets an important set of signal transduction proteins and has been extensively studied in Drosophila and Arabidopsis (Bergman and Siegel, 2003). These experiments reveal that Hsp90 suppresses phenotypic variation under normal conditions and releases this variation when functionally compromised by environmental stress, suggesting a 
constitutive role in adaptation. It has also been shown to exert pleiotropic effects on key developmental processes, meaning the function of $\mathrm{Hsp90}$ is linked to many measurable phenotypes.

From a drug discovery perspective, the interconnectedness of genes within these pathways and the robust nature of genetic networks make them useful for assessing interactions between drug targets and genes (Tong et al. 2001), as well as dissecting the mechanism of bioactive compounds in search of therapeutic applications (Carragher et al. 2012).

NMEs that inhibit, stimulate or otherwise interact with genes or pathways within these genetic networks can be designed or screened for. This allows for a changing paradigm of drug discovery. Instead of focusing on a single drug and its drug target, an integrated approach that encompasses entire gene networks can be used to develop a multi-drug strategy (Carragher et al. 2012). In this way, combinations of targeted agents could be effective in treating complex disease mechanisms such as tumours, where monotherapies are quickly subverted by clonal overgrowth of resistant cells.

\subsection{Synthetic compound libraries}

As mentioned previously, modern drug discovery often involves screening small molecules for their ability to bind to a protein target. An important parameter of drug discovery programmes then is the design and assembly of such libraries. Common sources for these small molecules include natural products harvested from biological sources and groups of synthetic compounds derived from diversity based synthesis. Each has its benefits and drawbacks as a source of such compounds.

Advanced methods for stereoselective and combinatorial organic synthesis have increased the efficiency with which small natural product-like molecules can be prepared (March and Smith, 2001; Brown and Poon, 2005). Using stereoselective techniques such as the Sharpless epoxidation (Takano et al. 1991), chemists are able to take simple precursors through to complex products without unwanted racemisation, allowing greater control of synthetic products. 
Combinatorial chemistry, wherein precursors are treated with a series of different reagents and conditions to produce libraries of varied compounds in a time and cost effective manner have become a primary source of NMEs for biological screening over the last 20 years (Koehn and Carter, 2005; Schreiber, 2000; Newman and Cragg, 2007). Using these techniques along with computer modelling, it is possible to rapidly and inexpensively build large libraries of compounds, whilst still maintaining control of the types of chemical structures produced (Gordeeva et al. 1992) in order to target a desired chemical structural space.

This is important for drug discovery because the random generation of large libraries, whilst impressive from an empirical point of view, has not resulted in increased drug discovery productivity (Newman and Cragg, 2007). Feher and Schmidt (2003) report that compared with natural products, synthetically derived compounds have a high attrition rate in the drug discovery process. They note that this is likely because reduced chirality (median chiral centres $=0$ ) and reduced structural rigidity (median rotatable bonds $=3$ ) are characteristic of combinatorial derived products in comparison to natural products (median chiral centers $=5$; median rotatable bonds $=1$ ) (Blunt et al. 2008).

This has seen the rise of diversity oriented combinatorial synthesis - compound collections that aim at coverage of a chemical space, instead of vast numbers of compounds (Ramasamay and Visuki, 2012; Burke and Schreiber, 2004). Such a paradigm allows structural diversity of NMEs, potentiating the screening of a range of biological activities within a library, instead of a series of closely related molecules which are likely to have similar activity profiles.

Candidate compounds for a synthetic library can be limited to 'drug like' compounds as a means to improve efficiency. This stems from the observation that many drugs share a series of properties, some of which are enumerated in Lipinski's rule of 5 (Lipinski et al. 2001) and the work of Arup Ghose et al. (1999) as including simple parameters such as molecular mass ( $<500 \mathrm{da}$.) and number of hydrogen bond donors $(<5)$ to more complex parameters such as molar refractivity $(40-130)$. Use of these criteria limits the number of compounds that must be assayed to manageable levels. 
The primary strength of synthetic libraries as a drug discovery tool is the ease with which they can be developed and screened. Diversity oriented synthesis (DOS) as exemplified in Stuart Schreiber's laboratory (Schreiber, 2000), can easily produce an array of greater than $92 \%$ purity in sufficient quantity for NMR spectroscopy identification in only 3-4 steps of synthesis. It is easier to develop a large range of natural product-like synthetic compounds than it is to squeeze them from limited quantities of, for example, obscure sea sponges. The down side of course is a lower 'hit ratio' compared to natural products (Gullo et al. 2003). Natural products have typically evolved alongside their targets, and are therefore highly likely to have bioactivity. By their existence these compounds must be the results of biological pathways: an organism wouldn't expend energy manufacturing metabolites if those metabolites didn't have a biological function. Marine natural products, because of the potential to be rapidly diluted in ocean waters, have typically evolved to be especially potent (De Vries and Beart, 1995).

However, the discovery and harvesting of such compounds is often fraught with difficulties and expense (Capson, 2009). Many promising natural product leads are taken from compounds associated with traditional or indigenous knowledge (Fabricant and Farnsworth, 2001). However, the pool of such knowledge is finite, and there are often thorny intellectual property issues involved with such discoveries (Capson, 2009; International Council for Science, 2002; Convention on Biological Diversity, 2010). In addition, most patent laws don't recognise 'natural discoveries' as patentable, and thus patent protection on natural products is only possible after some novel utility is shown (Barton and Emanuel, 2005; Rockman, 2004); the libraries themselves would be nonpatentable.

A further complication with the use of natural product libraries is the need to elucidate structures of each compound, impractical for a small laboratory, whereas the structure of synthetic compounds is easily derived from steps in their synthesis inherent in the DOS process. Construction of new natural product libraries is therefore difficult and their use usually limited to characterised, commercially 
available examples such as ENZO Life Science's Natural Product Library (Davenport et al. 2005). For this reason, most new libraries constructed for screening are synthetic in nature (Dolle and Nelson, 1999).

Producing viable quantities of a compound for testing and further development is also a critical consideration. In a research setting, it is often impractical or impossible to harvest quantities of a natural product, sufficient for research purposes, from its original source - often a rare organism itself. Therefore, in most cases a viable synthesis has to be developed if a lead compound is to move forward in development. In some cases, such as that of the microtubule stabilising anti-tumour agent peloruside A, such a synthesis can prove difficult (Evans et al. 2009). For synthetic compounds, the synthesis is known by definition.

\subsection{Saccharomyces cerevisiae}

A model organism is a species that is studied to understand biological phenomena, with the prospect that discoveries made in the model organism will provide insight into the analogous workings of other organisms, notably humans (Fields and Johnston, 2005). Appropriate model organisms allow investigation of the mechanisms of these phenomena with minimal difficulty and expense. Organisms which have a fully sequenced genome allow relative ease in identification of gene function and gene interactions and are part of the compendium of appropriate genetic models.

A particularly effective model organism for this purpose is the budding yeast Saccharomyces cerevisiae. There are several factors that contribute to S. cerevisiae's utility in this area (Forsburg, 2001). Firstly, there is the ease of genetic manipulation. Unlike 'higher' organisms, yeast strains such as S. cerevisiae and Schizosaccharomyces pombe can grow and divide both as haploid and diploid systems. As a result of this, recessive mutations can be easily recovered in haploid cells, but mutations in essential genes need not result in inviability in diploid cells.

Yeast is very amenable to many modern genetic manipulation techniques. They can be readily transformed via plasmid uptake and PCR based techniques (Forsburg, 2001). Due to homologous 
recombination during the sexual reproduction cycle, creating genomic-scale libraries of yeast strains, such as gene knockout and over-expression libraries, is possible (Winzeler, 1999; Rine, 1991; Tong et al, 2001). These allow systematic analyses - such as the development of comprehensive synthetic lethal redundant pathway networks - which are invaluable in discovery based strategies, wherein a specific target might not be known.

A variety of selective techniques are available when working with S. cerevisiae clones. For example, the carboxylase gene $A D E 2$, which catalyses a step in the 'de novo' adenine biosynthetic pathway, can be used to select for plasmid uptake: the build-up of the nucleotide precursor 5'-phosphoribosyl5-aminoimidazole and subsequent accumulation in the vacuole causes a red colour in the yeast colony, which can be alleviated by the addition of a functional copy of the gene on a plasmid (Jones and Fink, 1982; Heiter et al. 1985).

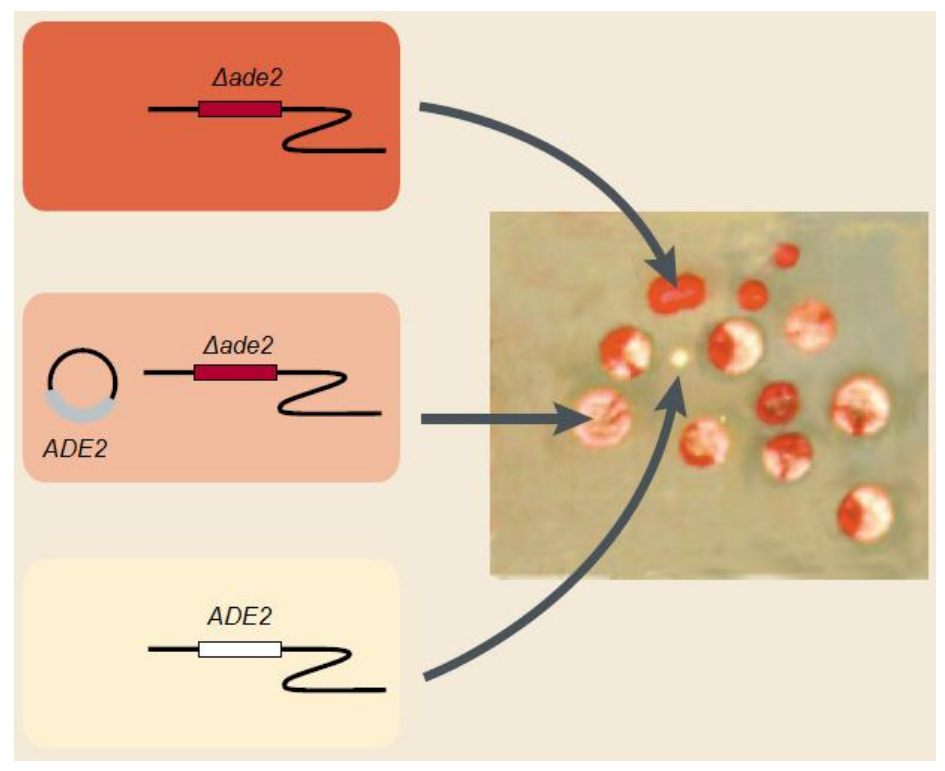

Figure 1.2 The ADE2 assay identifies cells with successful plasmid expression. $\triangle$ ade2 mutants display a red phenotype, which is rescued to a pink phenotype upon successful plasmid uptake and expression whilst WT cells remain white. The utility of this assay is that it allows recovery of all genotypes. This is useful when it is necessary to select for plasmid loss as well as plasmid uptake, for example when an auxotrophy selection must be reused at a later stage. Reprinted by permission from Macmillan Publishers Ltd: Nature Reviews Genetics, Forsburg, 2001.

Other important molecular techniques and concepts used with yeast include induced mutagenesis, temperature sensitive strains (Forsburg, 2001), genetic footprinting (Smith et al. 1995) and the generation of 'gene knockout' null mutants (Winzeler et al. 1999). 
Resistant mutant generation is a common strategy for identifying the target of compounds (Heidler and Radding, 1995). In this strategy, random mutagenesis is used to generate a mutant population which is selectively screened for resistance to a compound of interest. The mutation is then characterised to give insight into the mechanism of resistance, and therefore potentially the mechanism of action. A resistance mutant approach is discussed further in Chapter 4.

Temperature sensitive (TS) mutations are typically conditional loss of function alleles whose phenotypic effect is exacerbated when grown at a non-permissive temperature (Forsburg, 2001; Hartwell et al. 1970). This allows for the creation of mutations in essential genes that would otherwise be non-viable.

DNA footprinting is an older technique used to validate the functions of many hitherto putative genes at the dawn of the genomic age (Smith et al. 1995). Random insertional mutagenesis using transposition of a marked Ty1 transposable element is induced in a large population of cells, generating Ty1 insertional mutations at diverse sites. Such a population is then tested for phenotypic effects (such as red colouration of colonies - this technique was used to identify the function of the ADE2 gene mentioned above (Smith et al. 1995)). Relevant colonies are then selected, and the disrupted gene identified using Ty1 based PCR primers.

One of the most powerful strategies for determining genetic function both as regards to gene-gene and gene-drug interactions involves the use of 'knockout' mutants or single gene null mutants (Winzeler, 1999; Forsburg; 2001; Tong, 2001; Gaiever et al. 2003; Tong 2007; Nislow \& Gaiever, 2007; Ho et al. 2009; Roemer et al 2012). In this strategy a particular compound or gene mutation is assayed against a library of null mutants, attempting to identify interactions via an enhanced or mitigated phenotype. This approach is explored in Chapter 3.

The cell cycle in $S$. cerevisiae is very similar to the cell cycle in humans and is regulated by homologous proteins. The diploid sexual cycle means tests can be conducted for complementation, homologous recombination, or epistasis; impossible to do in haploid organisms because they require 
two copies of the gene. In addition, the $~ 6300$ genes that comprise $S$. cerevisiae's genome have been sequenced (Boone et al. 2007), even if the function of each is not fully understood. This understanding of the genome helps in investigating the potential of drug candidates because genetic interactions can be examined in a comprehensive, systematic way.

As a eukaryote, S. cerevisiae shares much of the complex internal cell structure of higher plants and animals, such as the Golgi apparatus, endoplasmic reticulum and mitochondria. There is considerable conservation of both cellular components as well as individual genes between yeast and human cells (Forsburg, 2001). The conservation of many mechanisms between S. cerevisiae and the cellular processes of humans mean that it is a good model for the exploration of genetic disease: Foury (1997) estimates that $30 \%$ of genes that are implicated in human disease have direct yeast orthologues. S. cerevisiae however does not have the high percentage of non-coding DNA ('junk' DNA) found in higher eukaryotes, which often make research difficult (Goffeau et al. 1996).

S. cerevisiae is a small single celled organism with a short generation time, allowing for the swift production and maintenance of multiple specimen lines at low cost. In addition, it is much easier to produce transgenic yeast cells through transformation than it is with higher order model organisms (Nickoloff and Hoekstra, 1998). This is in contrast with animal models such as Mus musculus which displays greater correspondence to human biology but is more difficult to manipulate, handle and maintain (Kyle and Hilton, 2005). S. cerevisiae therefore occupies an important niche in medical genetic research: enough homology to higher organisms for results to be meaningful, whilst being cost and effort effective enough to enable high throughput strategies.

This thesis utilises many of the tools herein described to characterise the target and mode-of-action of bioactive compounds derived from chemical synthesis and synthetic modification of natural products. Routine and leading-edge toxicity assays were used to screen the 254 compound library followed by a mutagenesis approach to create mutants resistant to FC-592, one of the most potent of these compounds. An advanced mapping strategy was used in an attempt to identify the 
resistance mediating genes. It is hoped that the activities of the studied compounds will produce lead compounds for further development as novel therapeutics or genetic probes. 


\section{Bioactivity Assays}

\subsection{Introduction}

\subsubsection{Background}

Interest in Saccharomyces cerevisiae remains high, both as a model organism to study basic eukaryotic cell biology and as a system for lead discovery of therapeutics, a process helped by the high conservation of cellular processes between yeast and mammalian cells (Hartwell, 2002). Use of S. cerevisiae in screens can potentiate the development of natural products (Barrett, 2002) and synthetic compounds (Parsons et al. 2006) to the clinic. This type of biological analysis is held to be vital in exploiting the potential of new lead compounds (Jefford \& Hartwell, 1998).

There are several approaches that can be taken when screening for bioactive compounds with $S$. cerevisiae based assays. The development of arrayed, genome-wide deletion libraries (Mager \& Winderickx, 2005) and the availability of arrayed chip based expression- (Zhou et al. 2005) and barcode- assays (Ho et al. 2009) have allowed genomic methods for dissecting bioactivity, with hierarchical clustering based on compound sensitivity profiles (Parsons et al. 2004). Haploinsufficiency profiling (Gaiever et al. 2003) can be used to identify target pathways. These are all automatable systems that are amenable to high throughput strategies. However, they are resource intensive; thus novel compound libraries should be pre-screened for bioactivity by simpler methodologies so resources are not wasted investigating inert compounds.

The simplest way to determine whether a candidate compound displays bioactivity is through an inhibition assay. This gives a first indication that the compound is in some way interacting with biological processes within the cell, preventing normal phenotypic expression. The chief advantages of this preliminary assay are that it is easy to perform in high throughput, and it is time and cost effective. An effective high throughput assay at this point allows us to narrow down the possible candidates to those with interesting biochemical effects. It is possible however, that more subtle variant phenotypes are missed through this type of assay. Because inviability or reduced growth is 
the phenotype being screened for in this case, less obvious effects such as altered expression of certain proteins might be missed. Screening is typically done at a high concentration for this reason: It stands to reason that any compound which affects a cell's normal phenotype will be deleterious to that cell's growth at a high enough concentration.

\subsubsection{The halo assay}

Ideally, the initial inhibition assay would be quantitative, easily automated, and compatible with high-throughput screening robotics. Typically a dose response against a range of concentrations in liquid media might be employed (Collinson \& Dawes, 1992; Broekaert et al. 1990). However, a particularly efficient assay for identifying inhibition without the need for complex dilution arrays is the halo assay (Gassner et al. 2007), a solid phase assay wherein yeast cells are seeded throughout a liquid agar nutrient medium. When the agar sets, compounds are robotically spotted atop the solid agar surface and allowed to diffuse. This creates a natural concentration gradient. After incubation, inhibitory compounds produce a distinctive 'halo' pattern in the yeast bloom, with more potent compounds producing larger halos.

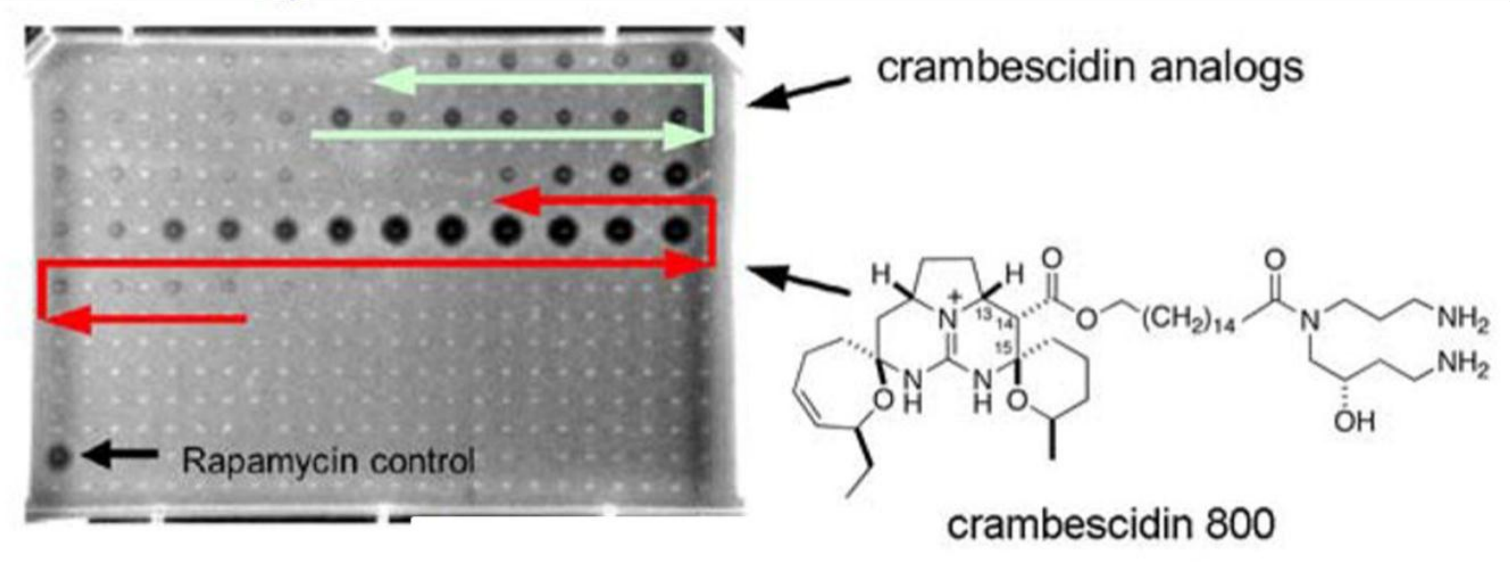

Figure 2.3 Distinctive 'halo' patterns show up around the locations of spotted inhibitory analogues of crambescidin. The pinned compounds show up as white spots on the surface of this plate. In this case fractions from HPLC-fractionation of the marine sponge Monanchora unguifera were spotted. Note the high potency of the crambescidin rich fractions in comparison to a rapamycin control spot. Reprinted with permission from American Chemical Society: Journal of Natural Products, Gassner et al. 2007. 


\subsubsection{The PDR Network}

S. cerevisiae is well known for its broad spectrum multidrug resistance (Balzi \& Goffeau, 1995). This has been linked to a series of genes within the pleiotropic drug resistance (PDR) network (Balzi \& Goffeau, 1991), and take the form of a complex genetic network of at least twenty genes underlying resistance to cytotoxic compounds and more generally implicated in membrane transport functions (Akache et al. 2004).

These genes can be grouped into three broad classes (Balzi \& Goffeau, 1995): ATP-binding cassette (ABC) membrane transport proteins such as SNQ2, PDR5, and YCFI; major facilitators superfamily membrane transport proteins such as ATRI and SGE1; and finally transcription factors including PDRI, PDR3, PDR7, PDR9, YAP1, and YAP 2, which regulate and control cellular responses to drug insult.

The PDR network causes obvious problems with drug discovery strategies utilising yeast: it can drastically reduce cytosolic concentrations of the candidate compounds making identification of potential bioactives more difficult. This is especially the case in inhibition type assays such as the halo assay where cellular concentration of the compound must remain high enough to prevent growth.

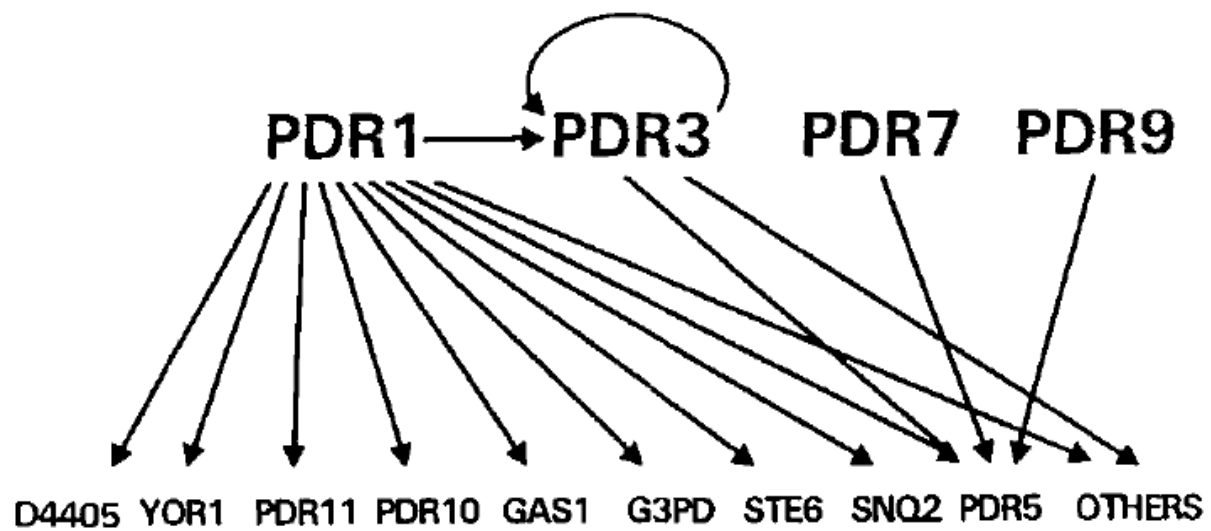

Figure 2.4 Regulatory transcription factors of the PDR network. Arrows represent regulatory interactions, wherein multiple S. cerevisiae regulators, including PDRI, PDR3, PDR7, and PDR9, control the expression of various membrane transport associated genes. Note the feedback mechanism associated with PDR3, whose promoter has been shown to have different binding sites activated by both PDR1p and PDR3p (Delahodde et al. 1995). Reprinted with permission from Springer Science: Journal of Bioenergetics and Biomembranes, Balzi \& Goffeau, 1995. 
The effect of the PDR network on an assay such as described could be quite deleterious. In a similar zone of inhibition assay (Meyers et al. 1992), PDR attenuated mutants displayed zones of inhibition that were over twice as big in inhibition tests for antimycin (15 ng applied compound), chloramphenicol (30 $\mu \mathrm{g}$ ), erythromycin (5 $\mu \mathrm{g}$ ) and lincomycin (60 ng). Other compounds (oligomycin (375 ng), ethidium bromide (150 ng)) displayed no increased potency in the PDR attenuated strains.

This strongly implies that some compounds are able to be bound by PDR associated membrane transporters and are thus not substrates for the PDR efflux pumps. It is clear however that an inhibition assay utilising a PDR competent strain risks significantly reduced sensitivity. This is a problem when available quantities of candidate compounds are limited, or concentration ranges are limited by solubility factors etc. There is little or no structural correlation between compounds which are substrates and those which aren't (Balzi \& Goffeau, 1995), meaning there is no way to predict what type of compounds might be missed as potential bioactives.

The sensitivity can be improved by the use of an attenuated PDR strain. In the above example (Meyers et al. 1992) the PDR5 membrane transporter gene is disrupted with the Tn5 transposon (Leppert et al. 1990), rendering a hypersensitive cell.

In the current study, attenuation is achieved through disruption of the PDR1 and PDR3 transcription factors. Since these transcription factors regulate multiple efflux pumps, affecting a broad array of genes (including the auto-regulating PDR3 feedback mechanism), the hypersensitivity effect should be correspondingly greater.

The yeast PDR network is analogous to the human multidrug resistance (MDR) system (Ueda et al. 1987). If the goal of the drug discovery program undertaken is human therapeutics, then an attenuated PDR based model is of less use. After all, a drug that only works in a non-drug resistant strain isn't going to be very good at treating diseases in multidrug resistant cells, and such compounds might have a high attrition rate during lead development. However, Miyamoto et al. (2002) report similar resistance to some drugs (such as reveromycin A) in MDR mammalian cells as 
PDR attenuated yeast cells. Whilst this study is not a comprehensive review of compounds, it suggests that PDR attenuated strains may make a better model for MDR mammalian cells than a wild type strain.

In addition, as mentioned in a previous chapter there are other goals to drug discovery projects such as the identification of genetic probes. Since the function of these tools is not predicated on their effect against wild type targets (further work can be done in PDR attenuated model strains), their utility is not compromised by being substrates for the PDR efflux pumps. It is therefore worthwhile trying to identify these compounds by using the PDR attenuated model.

The $\operatorname{pdr} 1 \Delta / \operatorname{pdr} 3 \Delta$ strain used in this project was constructed by PCR mediated disruption of the PDR1 and PDR3 loci from the y7092 background strain with the NatR resistance cassette and URA3 deletion cassette from the plasmid vector E. coli strains p4339 and pAG60 respectively, by Namal Coorey and Dr. David Bellows of the Chemical Genetics Laboratory, Victoria University of Wellington.

\subsubsection{The IRL Set of Synthetic Organic Compounds}

A screening library of novel organic compounds was obtained from Dr. Richard Furneaux of Industrial Research Limited, Gracefield, Lower Hutt. The library consists of 254 compounds consisting of (a) residual samples from a diverse array of research projects related to the development of novel pharmaceuticals and agrochemicals, including intermediates, by-products and final products, and (b) compounds derived by synthetic modification of New Zealand sourced diterpenoid natural products that had been donated to IRL by Professor PK Grant, University of Otago, on his retirement (Richard Furneaux, personal communication). 


\subsection{Aims and objectives}

The aim of this chapter is to develop a high throughput screening method for novel drug candidates, and characterise the inhibition profiles in PDR pump deficient strains, of at least one small molecule drug candidate. The key objectives are:

1. Develop and run a high throughput inhibition assay

2. Screen a library of compounds to identify bioactives

3. Characterise MIC and PDR profiles of these compounds

\subsection{Methods and materials}

\subsubsection{Yeast strains}

\begin{tabular}{|c|c|}
\hline Strain & Genotype \\
\hline Y7092 & 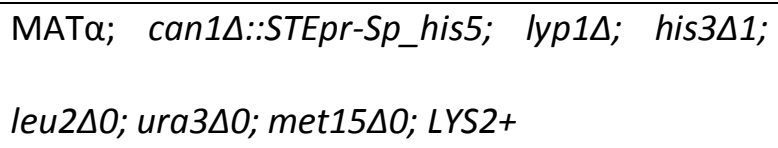 \\
\hline YCG326 & 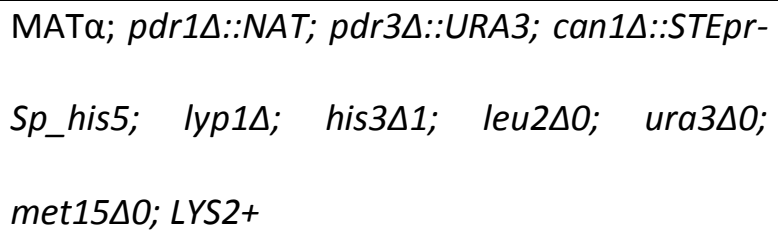 \\
\hline $\operatorname{pdr} 5 \Delta$ & 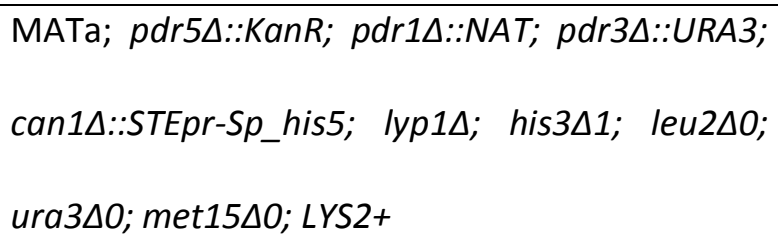 \\
\hline yor1 $\Delta$ & 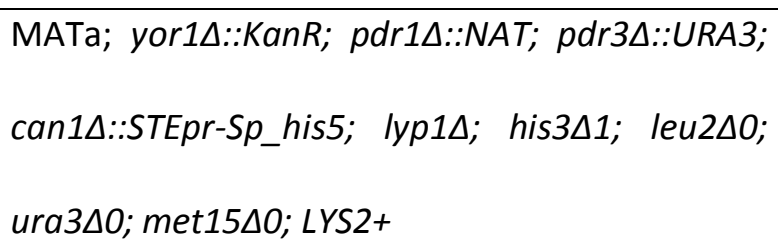 \\
\hline
\end{tabular}




\begin{tabular}{|c|c|}
\hline $\operatorname{snq} 2 \Delta$ & 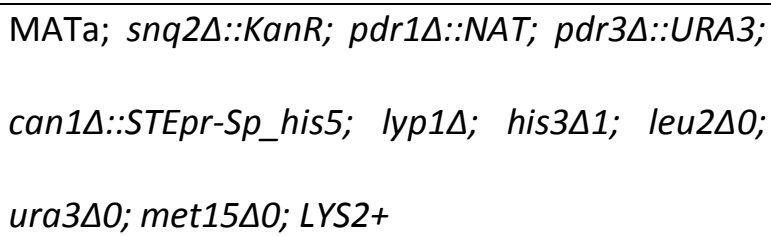 \\
\hline
\end{tabular}

\subsubsection{Growth media}

Yeast growth assays were performed in the following media formulations. All media was made up with distilled deionised $\mathrm{H}_{2} \mathrm{O}\left(\mathrm{ddH}_{2} \mathrm{O}\right)$ and autoclaved at $120^{\circ} \mathrm{C}$ for $20 \mathrm{~min}$. Sterile glucose was added to media post autoclave:

Yeast peptone dextrose (YPD): $1 \%(\mathrm{w} / \mathrm{v}$ ) yeast extract (Bacto), $2 \%(\mathrm{w} / \mathrm{v})$ peptone (Bacto), $2 \%(\mathrm{w} / \mathrm{v})$ D-glucose (SigmaAldrich).

Synthetic complete (SC): $0.17 \%$ bacto-yeast nitrogen base (Becton, Dickinson \& Company), $0.1 \%$ $(\mathrm{w} / \mathrm{v})$ monosodium glutamate $0.2 \%(\mathrm{w} / \mathrm{v})$ amino acid mix (stock (55.2 $\mathrm{g}$ total): $3 \mathrm{~g}$ adenine, $2 \mathrm{~g}$ arginine, $2 \mathrm{~g}$ uracil, $2 \mathrm{~g}$ inositol, $0.2 \mathrm{~g}$ para-aminobenzoic acid, $2 \mathrm{~g}$ alanine, $2 \mathrm{~g}$ asparagine, $2 \mathrm{~g}$ aspartic acid, $2 \mathrm{~g}$ cysteine, $2 \mathrm{~g}$ glutamic acid (MSG), $2 \mathrm{~g}$ glutamine, $2 \mathrm{~g}$ glycine, $2 \mathrm{~g}$ histidine, $2 \mathrm{~g}$ isoleucine, $10 \mathrm{~g}$ leucine, $2 \mathrm{~g}$ lysine, $2 \mathrm{~g}$ methionine, $2 \mathrm{~g}$ phenylalanine, $2 \mathrm{~g}$ proline, $2 \mathrm{~g}$ serine, $2 \mathrm{~g}$ threonine, $2 \mathrm{~g}$ tryptophan, $2 \mathrm{~g}$ tyrosine, $2 \mathrm{~g}$ valine), $2 \%$ glucose.

Synthetic deficient (SD) - Ura: As above except without uracil in the amino acid mix.

NAT: Where noted, $100 \mu \mathrm{g} / \mathrm{mL}$ (final concentration) nourseothricin (ClonNat, Werner Bioagents) was added to media after sterilization.

G418: Where noted, $200 \mu \mathrm{g} / \mathrm{mL}$ (final concentration) geneticin (G418, Invitrogen) was added to media after sterilization. 
Agar: Where noted, $2 \%(\mathrm{w} / \mathrm{v})$ select agar (Invitrogen) was added before sterilization (YPD) or autoclaved separately and added afterwards (all other media).

MOPS: Where noted, media is buffered to physiological pH with $25 \mathrm{mM}$ MOPS (Invitrogen) buffer (final concentration, $\sim \mathrm{pH} 7.0$ ).

\subsubsection{Compounds and chemicals}

As stated in the introduction section, the library of compounds used in this assay was provided by Dr. Richard Furneaux of Industrial Research Ltd, in $1.5 \mathrm{~mL}$ eppendorf tubes. These compounds were dissolved in pure dimethyl sulfoxide (DMSO, SigmaAldrich), to a concentration of 10 or $20 \mathrm{mg} \mathrm{mL}^{-1}$, depending on the mass of compound available (appendix 1). The molar concentrations ranged from $14.3 \mathrm{mM}-165 \mathrm{mM}$ with a median of $35.5 \mathrm{mM}$. Cycloheximide (SigmaAldrich) and Rapamycin (LC Laboratories) were also dissolved in DMSO at a range of concentrations for comparative analysis. All compounds were stored at $-20^{\circ} \mathrm{C}$.

\subsubsection{Halo assay}

A $20 \mathrm{~mL}$ overnight culture of YCG $326\left(\sim 16 \mathrm{~h}, 30^{\circ} \mathrm{C}\right.$ in shaking incubator) in SD-Ura broth media was initiated and cell titre determined via haemocytometer. The culture was then diluted to $1.0 \times 10^{8}$ cells $\mathrm{mL}^{-1}$ with fresh SC. SC agar media was produced as above and buffered to $\sim \mathrm{pH} 7.0$ with MOPS buffer with a final concentration of $25 \mathrm{mM}$. The low $\mathrm{pH}$ of SC media attenuates the bioactivity of many pharmacologically active compounds. Buffering of the media to near neutrality reverses this effect. MOPS buffer works best for solid phase assays, as the commonly used HEPES buffer produces gas bubbles in the plates after incubation. Media was cooled to $\sim 50^{\circ} \mathrm{C}$, ensuring it was not so hot as to kill cells $\left(<55^{\circ} \mathrm{C}\right)$, before cells are inoculated into media for a final concentration of $\sim 2 \times 10^{5}$ cells $\mathrm{mL}^{-1} .40 \mathrm{~mL}$ liquid media was poured into rectangular polystyrene omnitrays (Singer) and allowed to set. $50 \mu \mathrm{L}$ of each IRL compound was plated into 96 well $1 \mathrm{~mL}$ round bottom plates (SigmaAldrich). The first and last columns $(1 \& 12)$ and rows $(A \& H)$ were filled with DMSO to avoid evaporation effects in the experimental samples either in the plates themselves or after transfer to liquid phase 
assays. $2 \mu \mathrm{L}$ of each compound was pin transferred via pinning robot (CyBio, CyBi-Well Channel Pipettor fitted with P996 $2 \mu \mathrm{L}$ pins) in 96 well format onto an identifiable location on the plate and allowed to diffuse into agar for $5 \mathrm{~min}$. Plates were then incubated at $30^{\circ} \mathrm{C}$ for $24 \mathrm{~h}$. Photographs of plates (Canon Powershot S3-IS) were then taken using ZoomBrowser EX remote shooting software (Canon Inc, NY, USA) and contrast enhanced using ImageJ software (National Institutes of Health). Halos were measured in $\mathrm{mm}$ and subjectively graded as there was variation in quality of inhibition zones as well as size.

\subsubsection{Dose response assays}

Liquid media dose response assays were conducted to determine minimum inhibitory concentrations of each identified inhibitory compound. Serial dilutions of each compound were performed in DMSO at a half $\log ^{10}$ scale in 96-well reagent plates, along with DMSO (negative control) and cycloheximide (positive control). The plate format and molar concentrations used in each well are given in Appendix 1. A single YCG326 colony was inoculated into $3 \mathrm{~mL}$ of SC broth and incubated at $30^{\circ} \mathrm{C}$ on a $40 \mathrm{rpm}$ rotary drum overnight. Cell titre was determined via haemocytometer, diluted with fresh SC MOPS to a final concentration of $5 \times 10^{5}$ cells $\mathrm{mL}^{-1}$ and vortexed to create a homogeneous suspension. $100 \mu \mathrm{L}$ of media containing cells was dispensed (Eppendorf Xplorer Plus 8 Channel Electronic Pipette) into each well of a clear 96 well flat-bottom polystyrene cell culture plate (Interlab) and $1 \mu \mathrm{L}$ aliquots of each dilution were dispensed in triplicate using a multi-channel pipettor (Eppendorf Research Plus 8 Channel) into these wells, along with 1\% DMSO controls. Plates were vortexed at $1000 \mathrm{rpm}$ for $30 \mathrm{~s}$ to mix completely and incubated at $30^{\circ} \mathrm{C}$ for $\sim 18 \mathrm{~h}$. Following incubation, cultures were resuspended by vortexing at $1000 \mathrm{rpm}$ for $30 \mathrm{~s}$ and the absorbance measured at $590 \mathrm{~nm}$ in a spectrophotometer (EnVision 2102 Multilabel Plate Reader, Perkin Elmer, Waltham, Ma, USA). Residual growth (\%) was determined by comparing the turbidity in each condition against the mean absorbance for DMSO control (Abs590 (experimental)/ Abs590 DMSO (control)) $\times 100$ for each unique condition. 


\subsection{6. $\mathrm{pH}$ sensitivity assays}

Dose response assays were carried out to determine selected bioactive compounds' relative potency in differing $\mathrm{pH}$ conditions. A culture of YCG326 was grown overnight in $3 \mathrm{~mL} \mathrm{SC}$ broth $\left(30^{\circ} \mathrm{C}, 40 \mathrm{RPM}\right.$ on rotary drum). Cell titres were determined via haemocytometer, diluted to $5 \times 10^{5}$ cells $\mathrm{mL}^{-1}$ with fresh SC ( $\sim \mathrm{pH} 4.0)$ and SC MOPS (25 mM final concentration, $\sim \mathrm{pH} 7.0)$ in two different media solutions and vortexed to create a homogeneous suspension. $100 \mu \mathrm{L}$ aliquots of each media type were dispensed into cell culture plates as for dose response assays protocol. Dilutions (all final concentrations) of $1.13 \mathrm{mM}, 357 \mu \mathrm{M}, 113 \mu \mathrm{M}, 35.7 \mu \mathrm{M}, 11.3 \mu \mathrm{M}, 3.57 \mu \mathrm{M}$ and $1.13 \mu \mathrm{M}$ for FC-888, and $354.61 \mu \mathrm{M}, 112.13 \mu \mathrm{M}, 35.46 \mu \mathrm{M}, 11.21 \mu \mathrm{M}, 3.54 \mu \mathrm{M}, 1.12 \mu \mathrm{M}$ and $354.6 \mathrm{nM}$ for FC-592 were prepared. $1 \mu \mathrm{L}$ aliquots of each dilution were dispensed in triplicate into wells containing each media type, along with triplicate $1 \mu \mathrm{L}$ DMSO controls. Plates were incubated and measured as for the dose response assays protocol.

\subsubsection{PDR mutant sensitivity assays}

Dose response assays were carried out to determine the inhibition profiles of the identified inhibitory compounds FC-592 and FC-888 in PDR mutants. Yeast cultures of Y7092 (Wild Type), YCG326 ( $\Delta$ pdr1::NAT; $\Delta$ pdr3::URA3), yor1 $\Delta$, pdr5 $\Delta$ and snq2 $\Delta$ were grown overnight in $3 \mathrm{~mL}$ SC broth $\left(30^{\circ} \mathrm{C}, 40 \mathrm{RPM}\right.$ on rotary drum). Cell titres were determined via haemocytometer and diluted to $5 \times 10^{5}$ cells $\mathrm{mL}^{-1}$ with fresh SC. $100 \mu \mathrm{L}$ aliquots of media with cells were dispensed into cell culture plates as for dose response assays protocol. Dilutions of FC-888 and FC-592 were prepared as for $\mathrm{pH}$ sensitivity assay and dilutions of cycloheximide ( $\mathrm{CHX}$ ) prepared (final concentrations) at decrements of $10 \mu \mathrm{M}, 3.16 \mu \mathrm{M}, 1 \mu \mathrm{M}, 316 \mathrm{nM}, 100 \mathrm{nM}, 31.6 \mathrm{nM}$ and $10 \mathrm{nM} .1 \mu \mathrm{L}$ aliquots of each dilution were dispensed in triplicate into wells, along with triplicate $1 \mu \mathrm{L}$ DMSO controls. Plates were incubated and measured as for the dose response assays protocol.

\subsubsection{Solid state dose response assays}

A half-log dilution series was created in DMSO for FC-888 and FC-592 with final concentrations of $112.9 \mu \mathrm{M}, 35.7 \mu \mathrm{M}, 11.3 \mu \mathrm{M}, 3.57 \mu \mathrm{M}, 1.13 \mu \mathrm{M}, 357 \mathrm{nM}, 113 \mathrm{nM}$ \& $35.7 \mathrm{nM}$ along with $354.6 \mu \mathrm{M}$, 
$112.1 \mu \mathrm{M}, 35.5 \mu \mathrm{M}, 11.2 \mu \mathrm{M}, 3.55 \mu \mathrm{M}, 1.12 \mu \mathrm{M}, 355 \mathrm{nM} \& 112 \mathrm{nM}$ respectively. $3 \mu \mathrm{L}$ of each dilution, along with $1 \%$ DMSO and $100 \mathrm{nM}$ rapamycin controls, was aliquoted into $1.5 \mathrm{~mL}$ microcentrifuge tubes and $297 \mu \mathrm{L}$ molten SC Agar pipetted into the tubes, one at a time. The mixture was gently pipette mixed, avoiding bubble formation. The mixture was then transferred into a clear flatbottom 48-well plate (Interlab), again avoiding bubbles, and allowed to set. A culture of YCG326 was grown overnight in YPD, cell titre determined via haemocytometer and diluted to $5 \times 10^{5}$ with fresh YPD and cell suspension vortexed to ensure homogenization. $2 \times 2 \mu \mathrm{L}$ of this suspension was spotted into each well, allowed to diffuse for 5 min then incubated for $\sim 40 \mathrm{~h}$ at $30^{\circ} \mathrm{C}$. Photographs (Canon Powershot S3-IS) were then taken using ZoomBrowser EX remote shooting software (Canon Inc, NY, USA) and visually inspected to determine cell growth.

\subsubsection{Colony forming unit assays}

A YCG326 culture was grown overnight in $5 \mathrm{~mL} \mathrm{SC}\left(30^{\circ} \mathrm{C}, 40 \mathrm{RPM}\right.$ on rotating drum), cell titre determined via haemocytometer and diluted to $5 \times 10^{5}$ cells $\mathrm{mL}^{-1}$ with fresh SC. $1 \mathrm{~mL}$ aliquots of this cell suspension were dispensed into sterile $15 \mathrm{~mL}$ falcon tubes. $10 \mu \mathrm{L}$ dilutions (all final concentrations) of FC-592 (500 $\mu \mathrm{M})$, FC-888 $(1.12 \mathrm{mM})$, cycloheximide $(100 \mu \mathrm{M})$, and rapamycin (10 $\mu \mathrm{M})$ as well as a $1 \%$ DMSO control, were pipetted into individual tubes and vortexed to mix. The tubes were incubated at $30^{\circ} \mathrm{C}$ for $\sim 16 \mathrm{~h}$ on a rotating drum. The treated cells were transferred to sterile $1.5 \mathrm{~mL}$ microcentrifuge tubes (Eppendorf) and centrifuged at $13000 \mathrm{rpm}$ for $30 \mathrm{~s}$ (Eppindorf $5415 \mathrm{r}$ microcentrifuge). Supernatant was discarded and cells washed by vortexing in $1 \mathrm{~mL}$ sterile $\mathrm{ddH}_{2} \mathrm{O}$. This wash step was repeated twice, centrifuged a final time, $100 \mu \mathrm{L} d \mathrm{dH}_{2} \mathrm{O}$ added to the pellet and vortexed to mix. Cell titre of washed cells was determined via haemocytometer and diluted to $5 \times 10^{4}$ cells $\mathrm{mL}^{-1}$ with sterile $\mathrm{ddH} 2 \mathrm{O}$. $200 \mu \mathrm{L}$ pools of ddH2O were pipetted onto separate SC agar petri plates, and $20 \mu \mathrm{L}$ of each sample dispensed carefully into these pools, to give 1000 cells per plate. The pooled cells were evenly spread over the surface of the plates with the aid of a sterile glass rod and allowed to dry for $\sim 20 \mathrm{~min}$. The plates were then inverted and grown at $30^{\circ} \mathrm{C}$ for 
$\sim 48 \mathrm{~h}$, after which they were photographed (Canon Powershot S3-IS) using ZoomBrowser EX remote shooting software (Canon Inc, NY, USA) and visually inspected to determine cell growth.

\subsubsection{Liquid phase comparison assay}

In order to determine the efficacy of the halo assay method, a comparison assay was conducted in liquid media. A $20 \mathrm{~mL}$ overnight culture of YCG $326\left(\sim 16 \mathrm{~h}, 30^{\circ} \mathrm{C}\right.$ in shaking incubator) in SD-Ura broth media was initiated. Cell titre was determined via haemocytometer, and culture diluted to $5.0 \times 10^{5}$ cells $\mathrm{mL}^{-1}$ with fresh SC and buffered to $\sim \mathrm{pH} 6.5$ with MOPS buffer with a final concentration of 25 mM. $100 \mu \mathrm{L}$ aliquots of this cell suspension were dispensed in clear flat bottomed 96-well cell culture plates (Interlab). $2 \mu \mathrm{L}$ aliquots of each compound in the IRL compound library were pin transferred via pinning robot (CyBio, CyBi-Well Channel Pipettor fitted with P996 $2 \mu \mathrm{L}$ pins) into these wells, which were then incubated at $30^{\circ} \mathrm{C}$ for $\sim 18 \mathrm{~h}$. To determine cell density, absorbance was measured at $590 \mathrm{~nm}$ in a spectrophotometer (EnVision 2102 Multilabel Plate Reader, Perkin Elmer, Waltham, Ma, USA). Residual growth (\%) was determined by comparing the turbidity in each condition against the mean absorbance for DMSO control as described in the dose response assay protocol.

\subsection{Results}

\subsubsection{Halo assay}

Potential bioactives in the IRL synthetic organic compound set were screened by halo assay. 22 compounds were identified as inhibitory, showing bioactivity in PDR compromised yeast cells. 
Halo assay plates
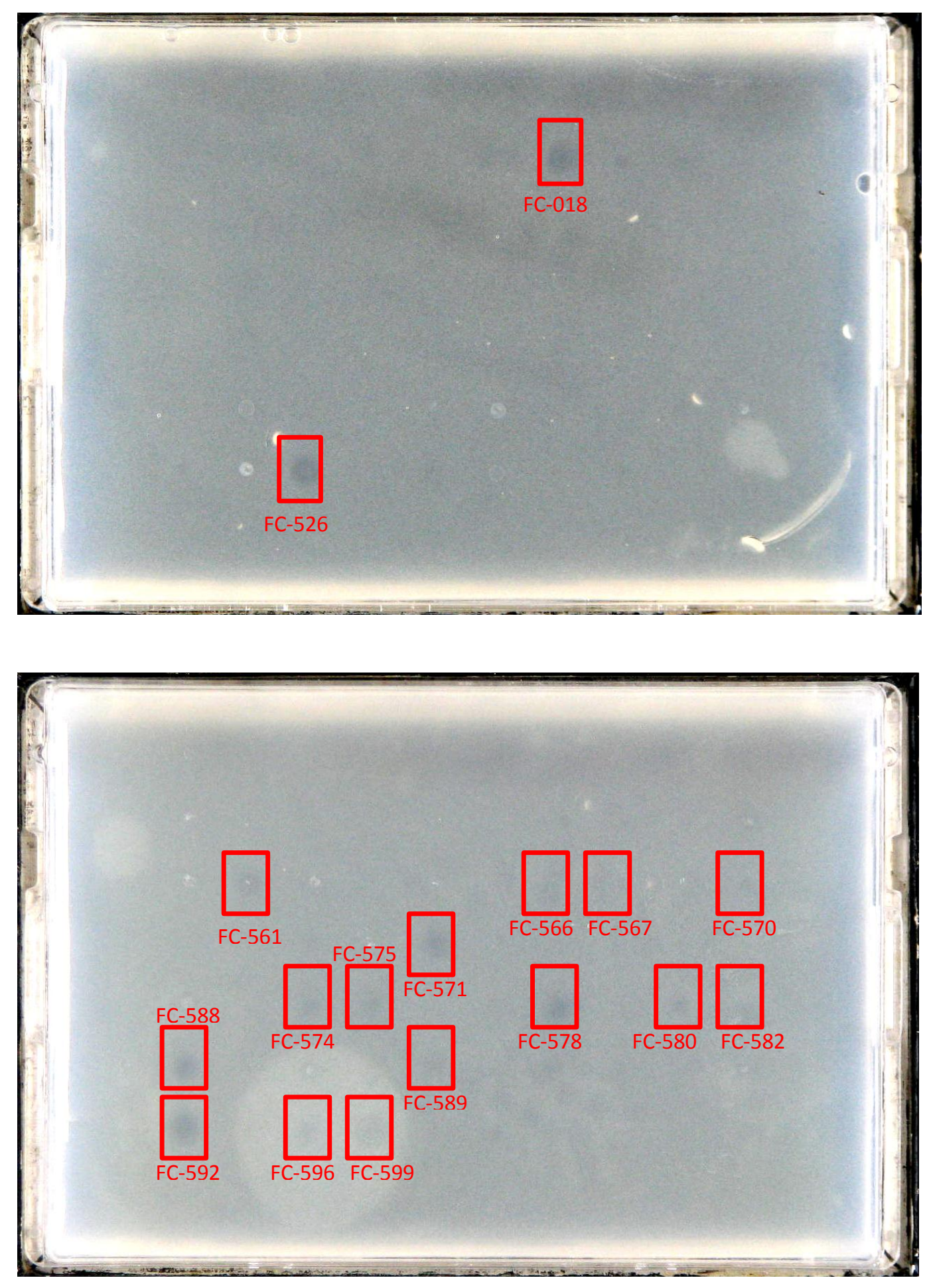

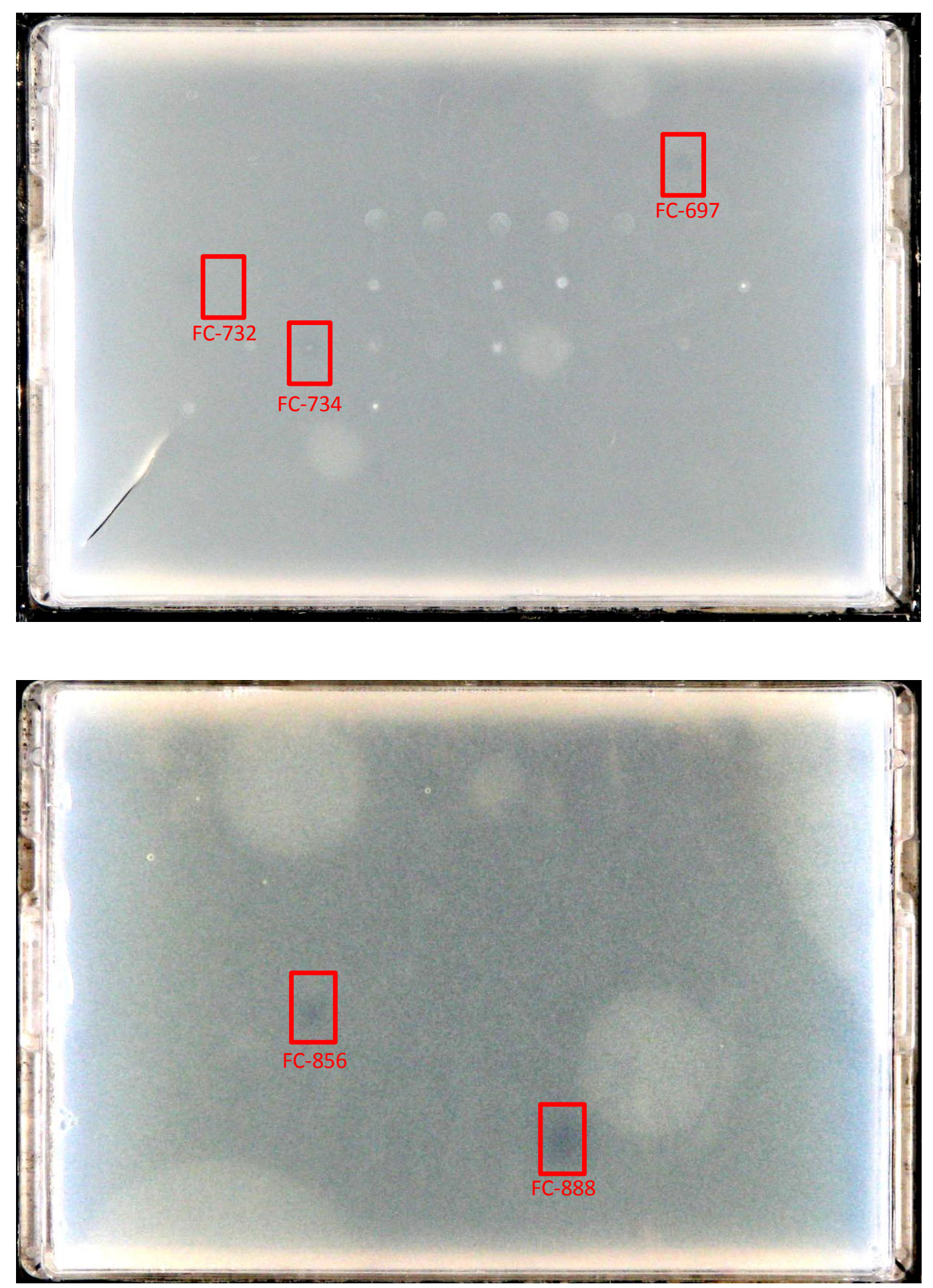


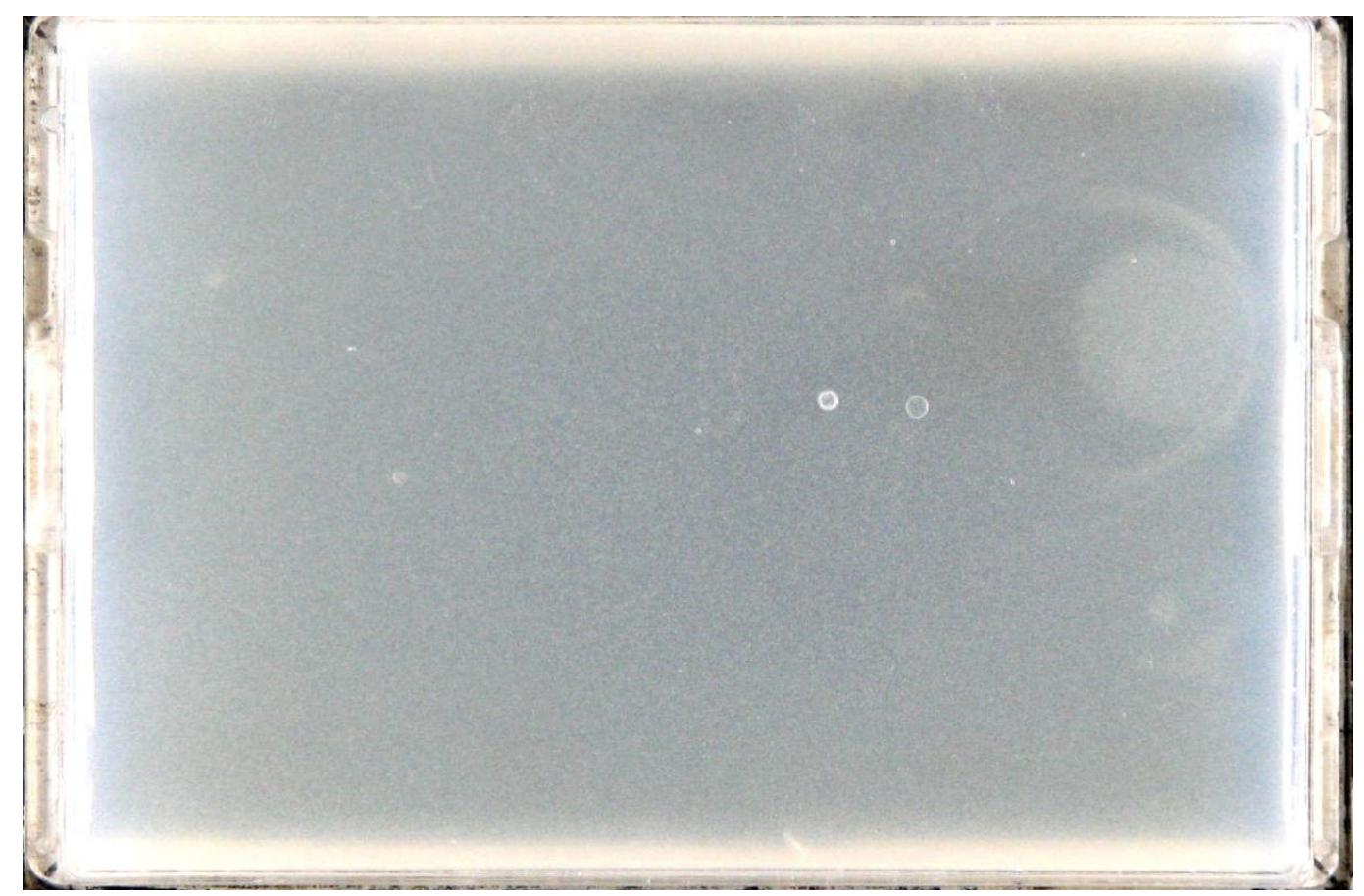

Figure 2.5 Halo assay shows inhibitory activity in novel compounds. $254 \mathrm{IRL}$ compounds (10 or $20 \mathrm{mg} \mathrm{mL}^{-1}$ ) were pinned onto 5 halo assay plates seeded with $2 \times 10^{5}$ cells $\mathrm{mL}^{-1}$ YCG326. 22 compounds produced visible halos indicating inhibitory bioactivity. Halos were ranked quantitatively $(\mathrm{mm})$ and qualitatively (1-5). Some halos had indistinct edges and were hard to see on photographs. These were ranked qualitatively only.

\section{Hallo assay hits}

\begin{tabular}{|l|c|c|c|c|c|c||}
\hline $\begin{array}{l}\text { IRL } \\
\text { Set } \\
\text { Hits }\end{array}$ & & & & & & \\
\hline & & & & & & \\
Catalogue \\
$\# \begin{array}{l}\text { Mass } \\
\text { (g) }\end{array}$ & $\begin{array}{c}\text { Molecular } \\
\text { mass }\end{array}$ & $\begin{array}{l}\text { Conc. } \\
\text { (mg/mL) }\end{array}$ & $\begin{array}{l}\text { Conc. } \\
\text { (mM) }\end{array}$ & $\begin{array}{l}\text { Halo } \\
\text { Assay } \\
\text { (mm) }\end{array}$ & $\begin{array}{l}\text { Score } \\
(1-5)\end{array}$ \\
\hline \hline FC-018 & 0.0124 & 262 & 10 & 38.1679 & 5.0 & 4 \\
\hline FC-526 & 0.0069 & $? ?$ & 10 & $? ?$ & 4.5 & 4 \\
\hline FC-561 & 0.0124 & 268 & 10 & 37.3134 & 4.0 & 2 \\
\hline FC-566 & 0.0138 & 268 & 10 & 37.3134 & - & 1 \\
\hline FC-567 & 0.0108 & $? ?$ & 10 & $? ?$ & - & 1 \\
\hline FC-570 & 0.0054 & 326 & 10 & 30.6748 & - & 1 \\
\hline FC-571 & 0.0114 & 382 & 10 & 26.178 & 4.5 & 3 \\
\hline FC-574 & 0.0171 & 308 & 20 & 64.9351 & - & 2 \\
\hline FC-575 & 0.0159 & 282 & 10 & 35.461 & - & 2 \\
\hline FC-578 & 0.0049 & $? ?$ & 10 & $? ?$ & 4.5 & 3 \\
\hline FC-580 & 0.0174 & 264 & 20 & 75.7576 & 3.0 & 2 \\
\hline FC-582 & 0.0062 & 330 & 10 & 30.303 & - & 1 \\
\hline FC-588 & 0.0081 & 248 & 10 & 40.3226 & 4.0 & 3 \\
\hline FC-589 & 0.0174 & 322 & 20 & 62.1118 & - & 1 \\
\hline
\end{tabular}




\begin{tabular}{|c|c|c|c|c|c|c|} 
FC-592 & 0.0079 & 282 & 10 & 35.461 & 5.0 & 4 \\
\hline FC-596 & 0.0135 & 308 & 10 & 32.4675 & - & 2 \\
\hline FC-599 & 0.0102 & 280 & 10 & 35.7143 & - & 2 \\
\hline FC-697 & 0.0134 & 363 & 10 & 27.5482 & 3.5 & 2 \\
\hline FC-732 & 0.0091 & 142 & 10 & 70.4225 & - & 1 \\
\hline FC-734 & 0.0028 & 415 & 10 & 24.0964 & - & 1 \\
\hline FC-856 & 0.0010 & 174.5 & 10 & 57.3066 & 4.0 & 3 \\
\hline FC-888 & 0.0223 & 177 & 20 & 112.994 & 5.5 & 5 \\
\hline
\end{tabular}

Table 2.1. Data on IRL compounds recording hits in halo assay. Halo size, which scales with compound potency (Gassner et al. 2007), shows a range among hit compounds of the IRL synthetic library set. Of particular note are FC-018, FC-526, FC592 and FC-888, which display large, defined halos. Many hits, such as FC-567, produced only minimal halos, with indistinct edges. For this reason they were assessed qualitatively.

Of 254 compounds in the IRL synthetic compound library, 22 displayed inhibitory activity in the halo assay. This translates to a hit rate of $8.66 \%$. Typical drug discovery programmes utilising synthetic combinatorial libraries have produced a hit rate of less than 1\% (Verheij \& Robeson, 2002), indicating the abnormally high bioactivity ratio of compounds within this library.

\subsubsection{Dose response assays}

Half log dose response assays were carried out using YCG326 in SC MOPS to determine potency of inhibitory compounds identified in the halo assay. Molecular data for three compounds, FC-526, FC567 and FC-578, was unavailable. For this reason, molar concentrations could not be calculated for these compounds. Since these would require time and effort to determine, these compounds were discarded at this point. A further two compounds, FC-018 and FC-734 did not show complete inhibition even at the highest concentrations. In the case of FC-018, this was despite a strong indication of inhibitory activity in the halo assay.

Minimum inhibitory concentrations ranged from $\sim 11.21 \mu \mathrm{M}$ for FC-592 to $\sim 650 \mu \mathrm{M}$ for FC-574. This compares to MICs of typical antifungals such as amphoteracin B (1-4 $\mu \mathrm{M})$ and fluconazole (64 $\mu M)($ Markovich et al. 2004), although these were not established in $\triangle P D R$ cell lines. The two most potent compounds, FC-592 and FC-888 were selected for further study. 


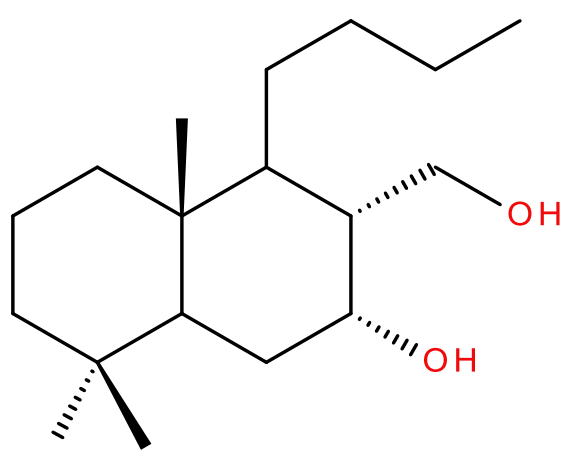

Figure $2.6 \quad$ FC-592: $\quad(2 R, 3 R, 4 a R)-4-$ butyl-3(hydroxymethyl)-4a,8,8-trimethyl-decalin-2-ol is a synthetically modified diterpine natural compound from New Zealand.

FC-888

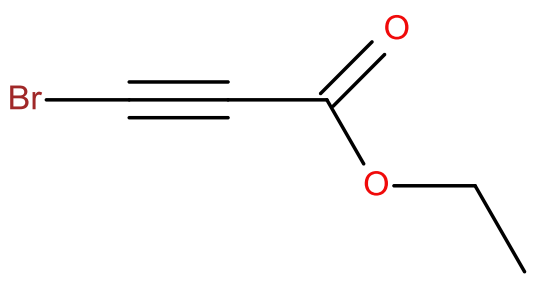

Figure 2.7 FC-888: ethyl 3-bromoprop-2-ynoate is a synthetic compound developed by IRL in Gracefield, New Zealand 


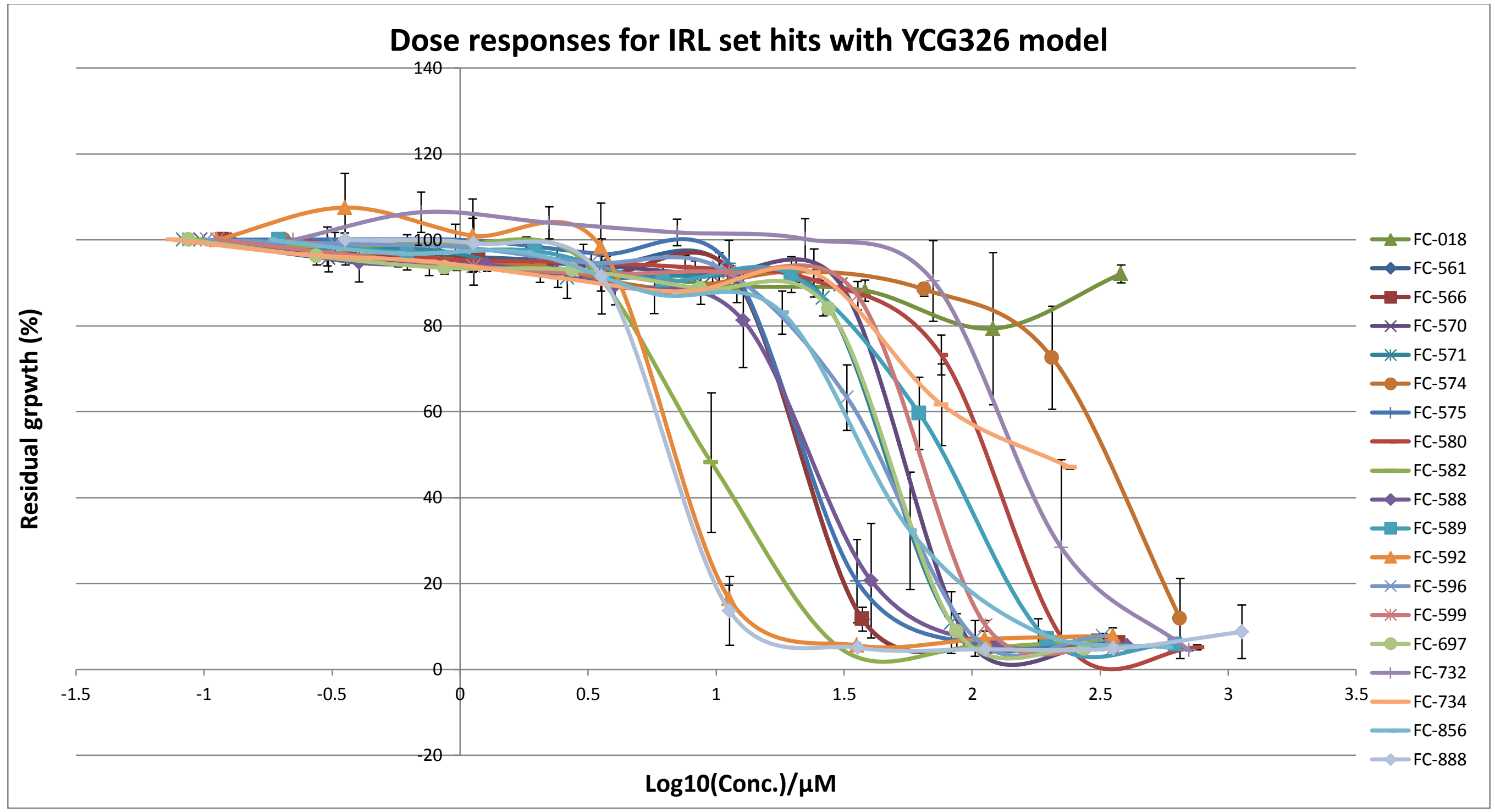

Figure 2.819 compounds from the IRL library were assayed via dose response for inhibitory activity in the YCG326 strain of yeast. Of these, 17 displayed complete inhibition within the concentration ranges tested. FC-018 did not display any inhibitory activity in the tested range (<381.68 $\mu \mathrm{M})$, despite showing as a strong hit in the halo assay. FC-592 and FC-888 were the most potent compounds tested, displaying MICs of $10 \mu \mathrm{M}$. 


\begin{tabular}{|c|c|}
\hline $\begin{array}{l}\text { IRL Set } \\
\text { Compound } \\
\text { Potency }\end{array}$ & \\
\hline Catalogue \# & $\begin{array}{l}\text { Minimum Inhibitory Concentration } \\
(\mu \mathrm{M})\end{array}$ \\
\hline \hline FC-518 & $>380$ \\
\hline FC-526 & $? ?$ \\
\hline FC-561 & 37.31 \\
\hline FC-566 & 37.31 \\
\hline FC-567 & $? ?$ \\
\hline FC-570 & 97.00 \\
\hline FC-571 & 82.78 \\
\hline FC-574 & 649.35 \\
\hline FC-575 & 35.46 \\
\hline FC-578 & $? ?$ \\
\hline FC-580 & 239.57 \\
\hline FC-582 & 30.30 \\
\hline FC-588 & 40.32 \\
\hline FC-589 & 196.41 \\
\hline FC-592 & 11.21 \\
\hline FC-596 & 324.68 \\
\hline FC-599 & 112.94 \\
\hline FC-697 & 87.12 \\
\hline FC-732 & 222.70 \\
\hline FC-734 & $>240$ \\
\hline FC-856 & 57.31 \\
\hline FC-888 & 11.29 \\
\hline 25150 & \\
\hline
\end{tabular}

Table 2.2 17 MICs of the IRL compound library. Compounds from the IRL library showed complete inhibition against YCG326 at the concentration ranges tested. 


\subsection{3 pH dependant inhibitory activity assay for FC-592 and FC-888 in YCG326.}

The effects of varying $\mathrm{pH}$ conditions on the biological activities of FC-592 and FC-888 were assayed via liquid dose response with a PDR attenuated yeast strain. Both compounds were tested against YCG 326 in SC media ( p pH 4.0) and SC MOPS media ( $\sim$ pH 7.0). No significant difference in inhibition profiles or MICs was observed between low pH and neutral pH conditions in either FC-592 or FC-888 indicating that the biological activities of neither compound are $\mathrm{pH}$ dependant.

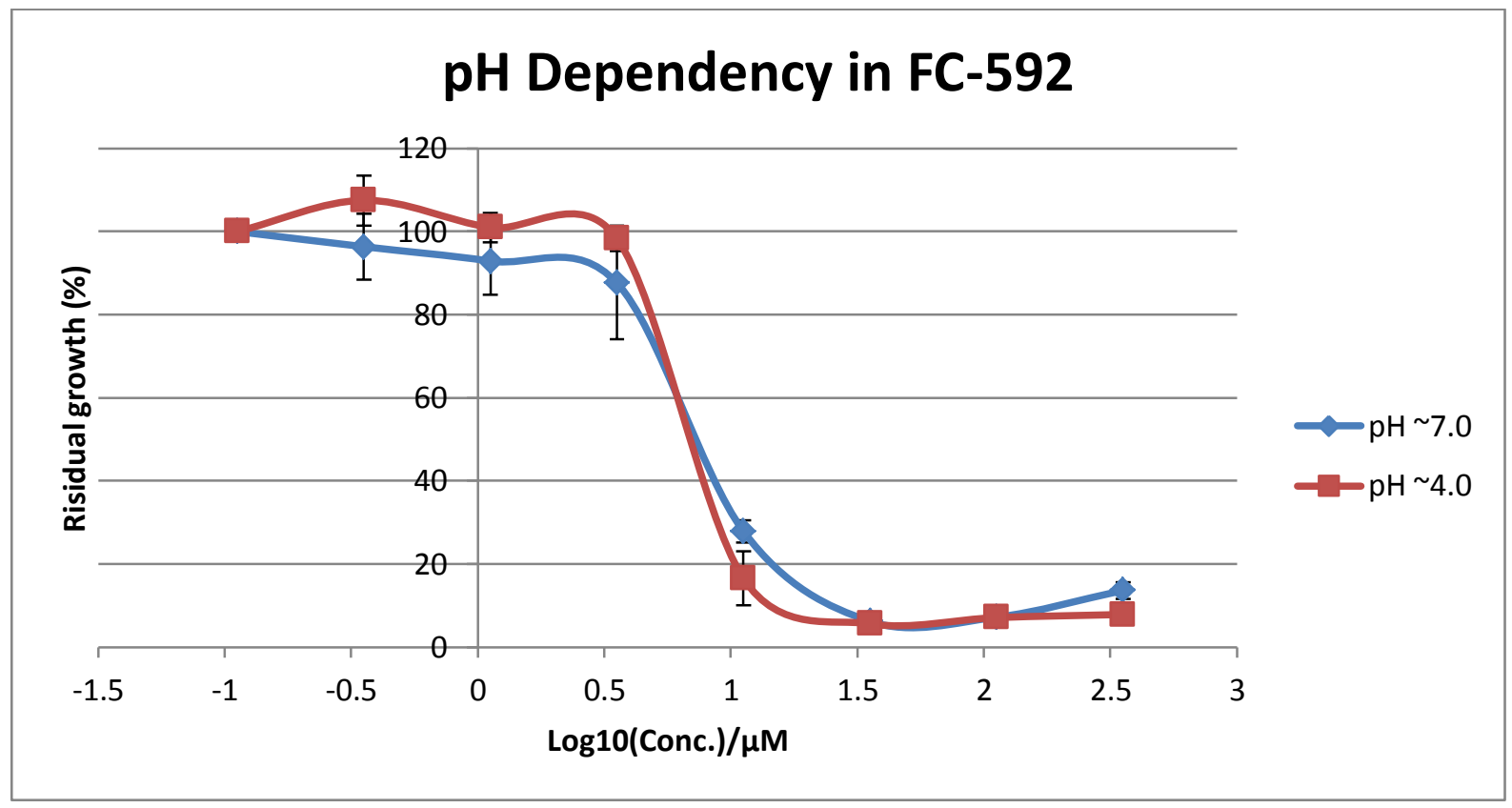

Figure 2.9 FC-592 does not show pH dependent biological activity in a YCG326 based dose response assay. Similar inhibition profiles are seen in SC ( ph 4.0) and SC MOPS ( pH 7.0) media, as well as displaying similar MICs of $\sim 10 \mu \mathrm{M}$. 


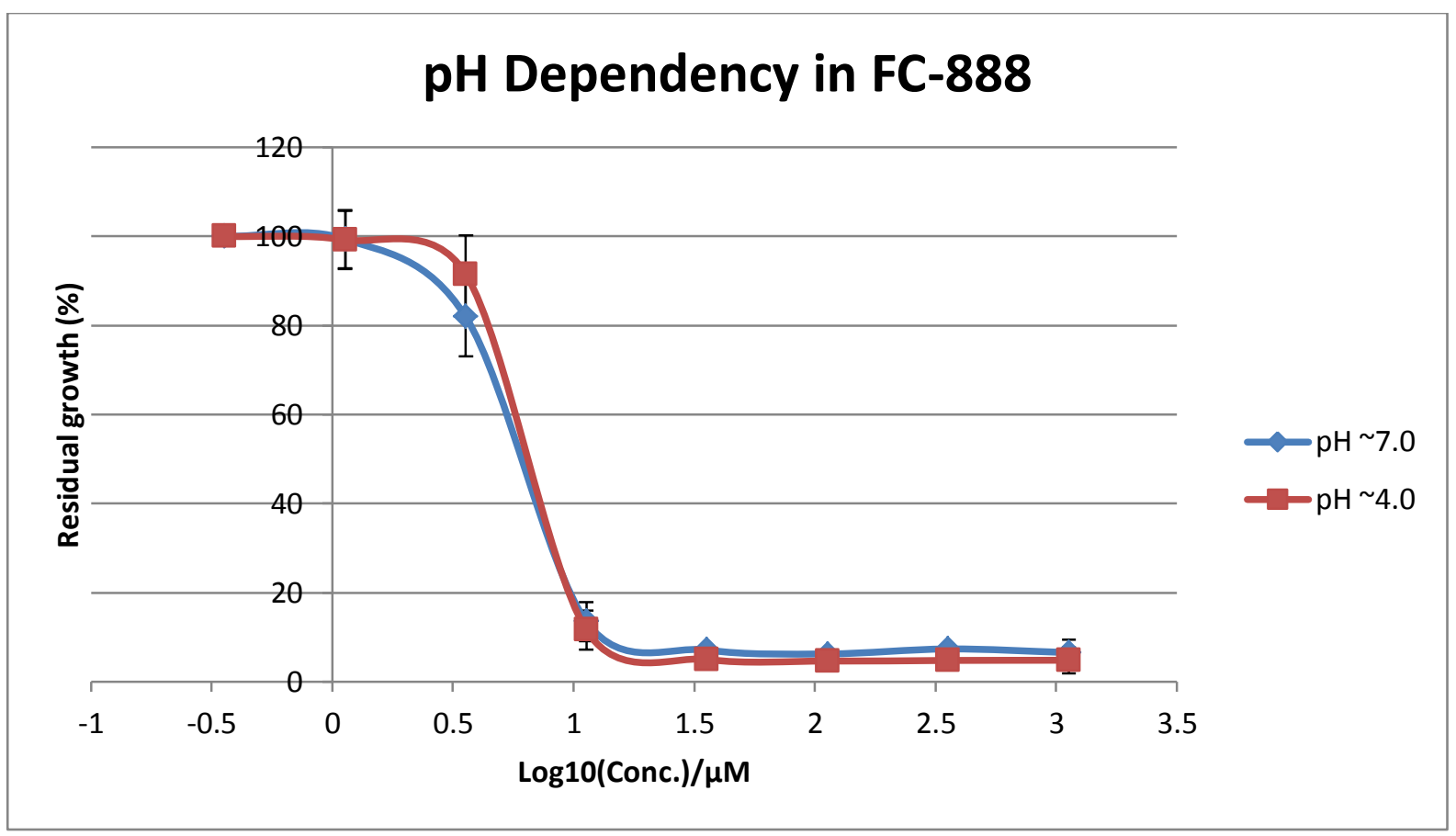

Figure 2.10 FC-888 showed no significant difference in inhibition profile in differing pH conditions in a YCG326 based liquid dose response assay. Similarly to FC-592, this suggests that FC-888 does not have pH dependant biological activity, displaying an MIC of $\sim 10 \mu \mathrm{M}$ in both SC ( pH4.0) and SC MOPS ( pH 7.0) media.

\subsubsection{PDR dependant inhibition assay}

Liquid dose response assays were performed using 5 yeast strains. Yor $1 \Delta, \operatorname{pdr} 5 \Delta$ and snq2 $\Delta$, obtained from the Boone Array, as well as YCG326 ( $\Delta \mathrm{pdr} 1 ; \Delta \mathrm{pdr} 3)$ and a wild type control, Y7092, were assayed against FC-592, FC-888 and cycloheximide (CHX, as a known substrate for the PDR pumps) as a control to determine the PDR network's effect on each drug. 


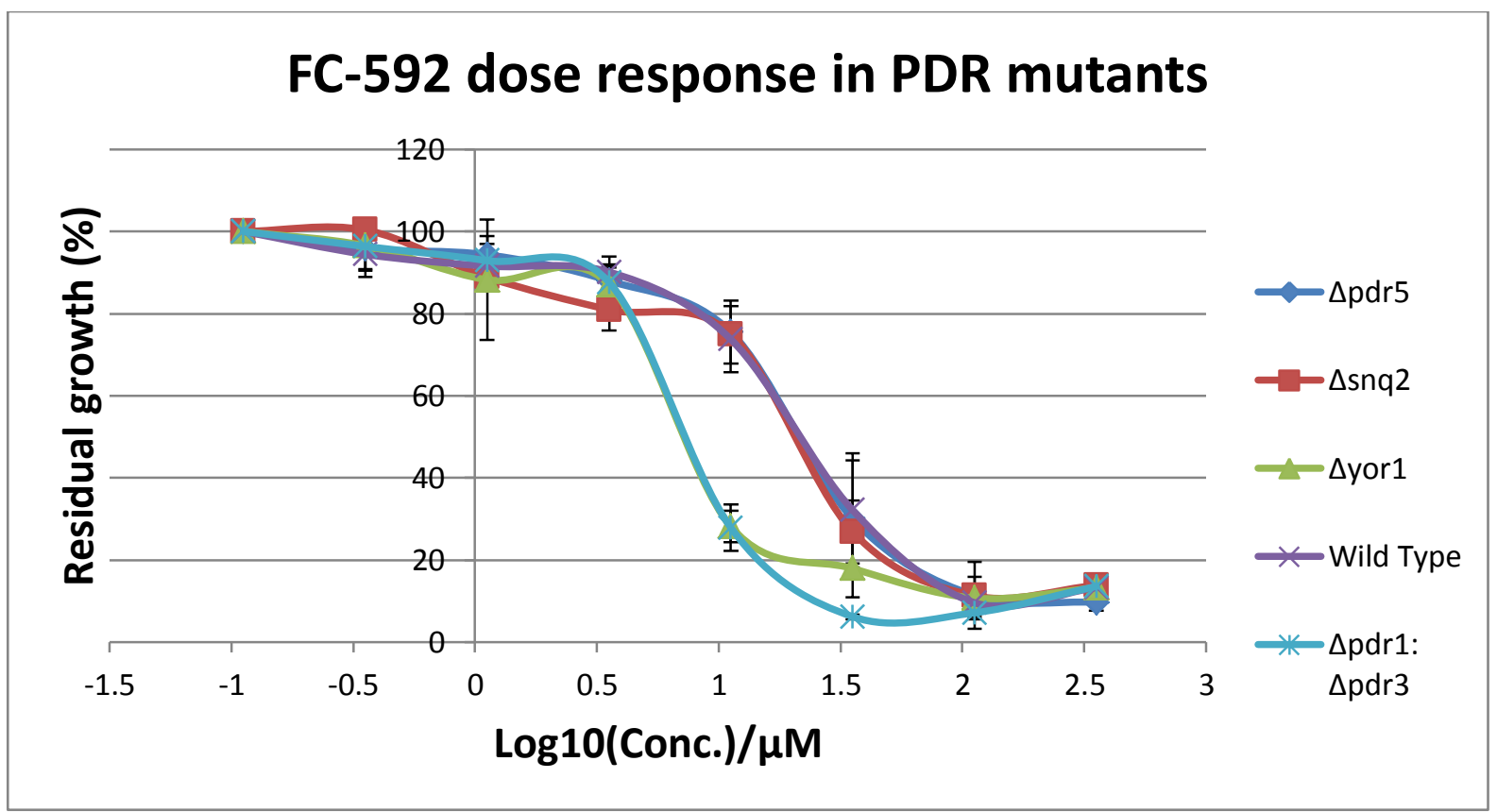

Figure 2.11 FC-592 shows differential sensitivity to PDR competent and PDR attenuated cells. FC-592 was 3-fold more potent in $\Delta$ yor1 cells and YCG326 cells, with MICs of $\sim 10 \mu \mathrm{M}$. It showed no significant difference in comparison to WT cells for $\Delta$ snq2 and $\Delta$ pdr5 cells (MIC $\sim 35 \mu \mathrm{M}$ ). This strongly suggests that FC-592 is a substrate for the membrane ATP-binding cassette (ABC) transporter YOR1p, which is regulated by the PDR1 and PDR3 transcription factors.

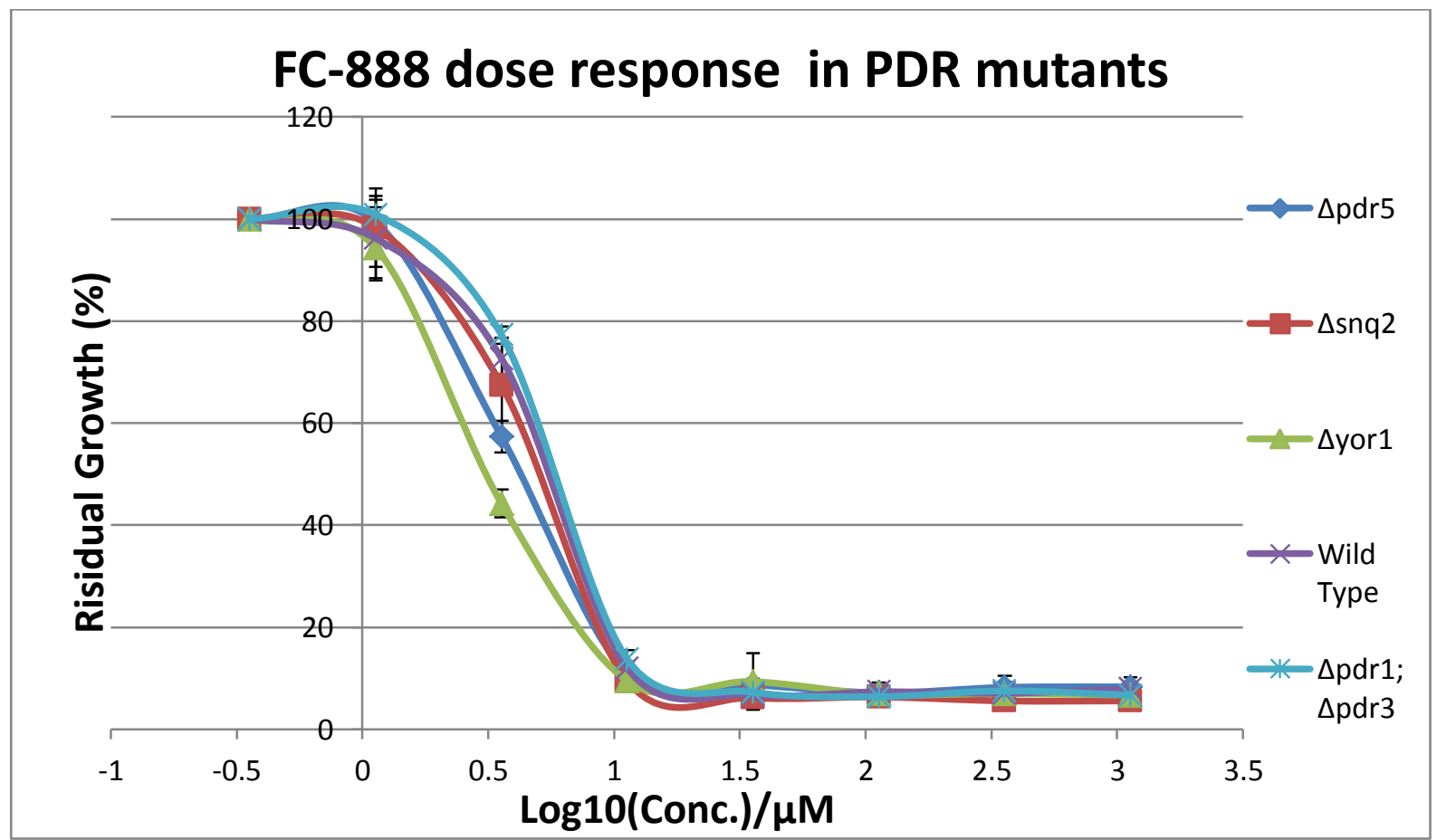

Figure 2.12 In contrast to FC-592, FC-888 did not show differential sensitivity in the PDR dose response assay. No significant difference was seen in sensitivity to FC-888 between PDR competent WT cells and PDR attenuated cells, including $\Delta \mathrm{pdr} 5, \Delta \mathrm{snq} 2, \Delta$ yor1 and YCG326. This suggests that FC-888 is not a substrate for the PDR mediated efflux pumps. 


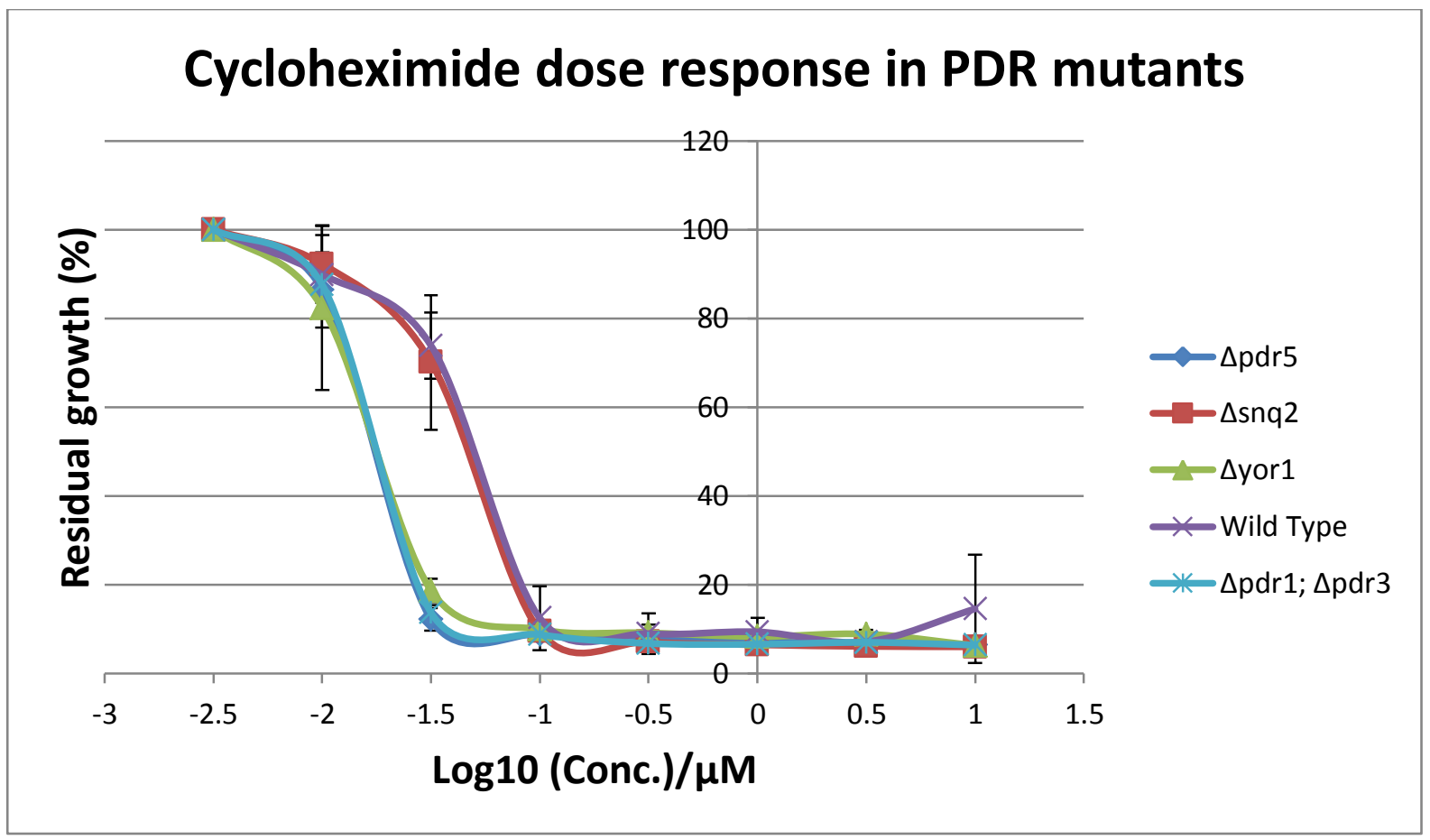

Figure 2.13 Cycloheximide (CHX), a known substrate for PDR mediated efflux pumps (Balzi et al. 1987), serves as a positive control for the PDR sensitivity assay. $\Delta \mathrm{pdr} 5$ and $\triangle$ yor1 strains, along with the PDR attenuated YCG326 strain, displayed increased sensitivity to CHX compared to a WT strain. The $\triangle$ snq2 strain did not show increased sensitivity over the wild type, suggesting the Snq2p membrane ATP-binding cassette (ABC) transporter is not involved in CHX efflux. These results, which follow expectations from the literature (Alamgir et al. 2010), show that this assay is effective in identifying PDR substrates.

Results from this assay indicated that FC-592 is a substrate for the PDR mediated efflux network. Specifically, evidence suggests that the Yor1p ATP-binding cassette (ABC) transporter, mediated by PDR1 and PDR3 transcriptions factors, is responsible for FC-592 efflux. This is suggested by three-fold increased sensitivity to FC-592 seen in yor1 $\Delta$ and YCG326 ( $\Delta$ pdr1; $\Delta p d r 3)$ strains compared to a wild type. Inability to efflux the compound means higher cytoplasmic concentrations of FC-592 are therefore likely to build up in these strains. This sensitivity was not seen in snq $2 \Delta$ and $p d r 5 \Delta$ strains, indicating FC-592 has no affinity for either of these efflux pumps.

No significant difference in sensitivity to FC-888 was seen in the PDR competent and PDR attenuated strains. This suggests that FC-888 is either not a substrate, or a very poor substrate for the PDR mediated efflux pumps. 


\subsubsection{Solid phase dose response}

In order to detect any differences in potency for FC-592 and FC-888 based on assay type, a solid phase dose response assay was conducted to compare with liquid phase dose response results. Halflog decrements of each compound were incorporated into SC Agar media in 48-well plate format along with $1 \%$ DMSO and $100 \mathrm{nM}$ rapamycin controls. Dilute cell suspensions of YCG326 were spotted onto each well to test sensitivity.

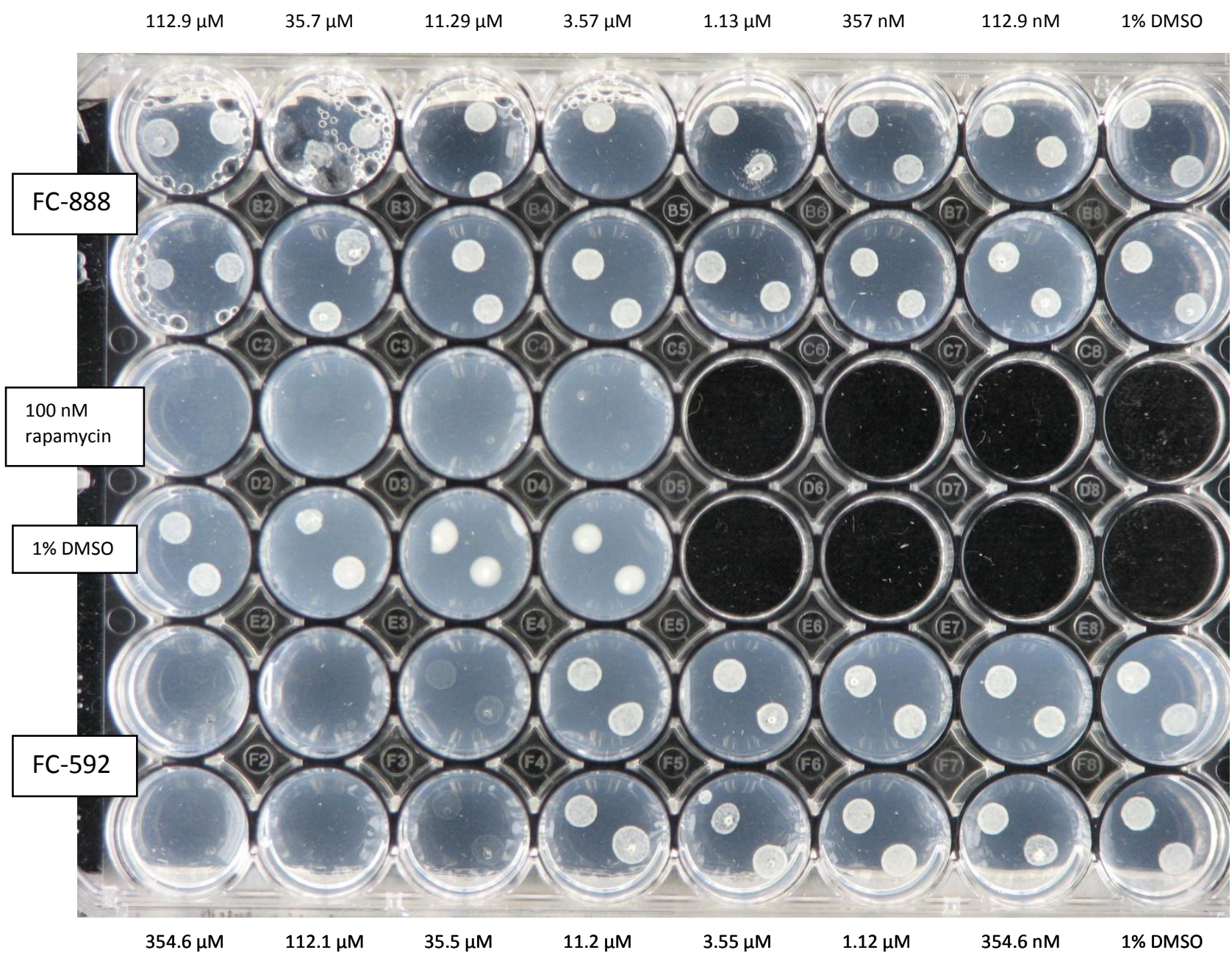

Figure 2.14 Solid state dose responses reveal the sensitivity of YCG326 yeast cells to FC-592 and FC-888 on agar media. Rows A and B consist of FC-888: these cells showed no inhibition at any concentration level. Rows E and F consist of FC592: cells were entirely inhibited at concentrations of $112.1 \mu \mathrm{M}$ and above, whilst growth is mostly inhibited at $35.5 \mu \mathrm{M}$. Row C consists of $100 \mathrm{nM}$ rapamycin as a positive control, and row D consists of $1 \%$ DMSO as a negative control. Complete inhibition was seen in positive controls, whilst full growth was seen in the negative controls, showing the assay worked as expected.

The solid phase dose response assay revealed the severely attenuated potency of FC-888 in solid phase. Whilst FC-888 inhibited growth at $~ 11 \mu \mathrm{M}$ in liquid phase assays, no inhibition was seen even at the highest concentration $(112 \mu \mathrm{M})$ in the solid phase assay. This raises interesting questions 
about cellular uptake of this compound, especially considering strong inhibition was observed for this compound in the solid phase halo assay.

FC-592 also showed some attenuated activity compared to the liquid phase assay, although not nearly to the same degree. The compound still inhibited growth at $35.5 \mu \mathrm{M}$, although growth still occurred at $11.2 \mu \mathrm{M}$. This indicates an MIC somewhere between 1-3 fold lower than the same compound in liquid media. For drug saving considerations, this means that further work with this compound will be more efficient in liquid than on agar based media.

\subsubsection{Liquid phase comparison assay}

To determine the efficacy of the halo assay, a comparison was made to a more traditional technique, using a liquid media inhibition assay. The IRL compound library was pinned into SC MOPS media containing YCG326 cells and grown for $\sim 18 \mathrm{~h}$. Optical absorbance was used to determine growth rate, with residual inhibition percentage $(100-(\mathrm{Abs}(\operatorname{Exp}) / \mathrm{Abs}(\operatorname{Cont}) \times 100)$ of at least $20 \%$ being considered inhibitory. Figure 2.15 displays the compounds identified as inhibitory in this assay. There were a far greater number of bioactive compounds identified here than there were from the halo assay. However FC-592 and FC-888 had already been selected based on previous results for further investigation, and so the newly identified bioactive compounds such as FC-017 and FC-517 were not explored further at this time. However, these remain a possibility for future investigation. 


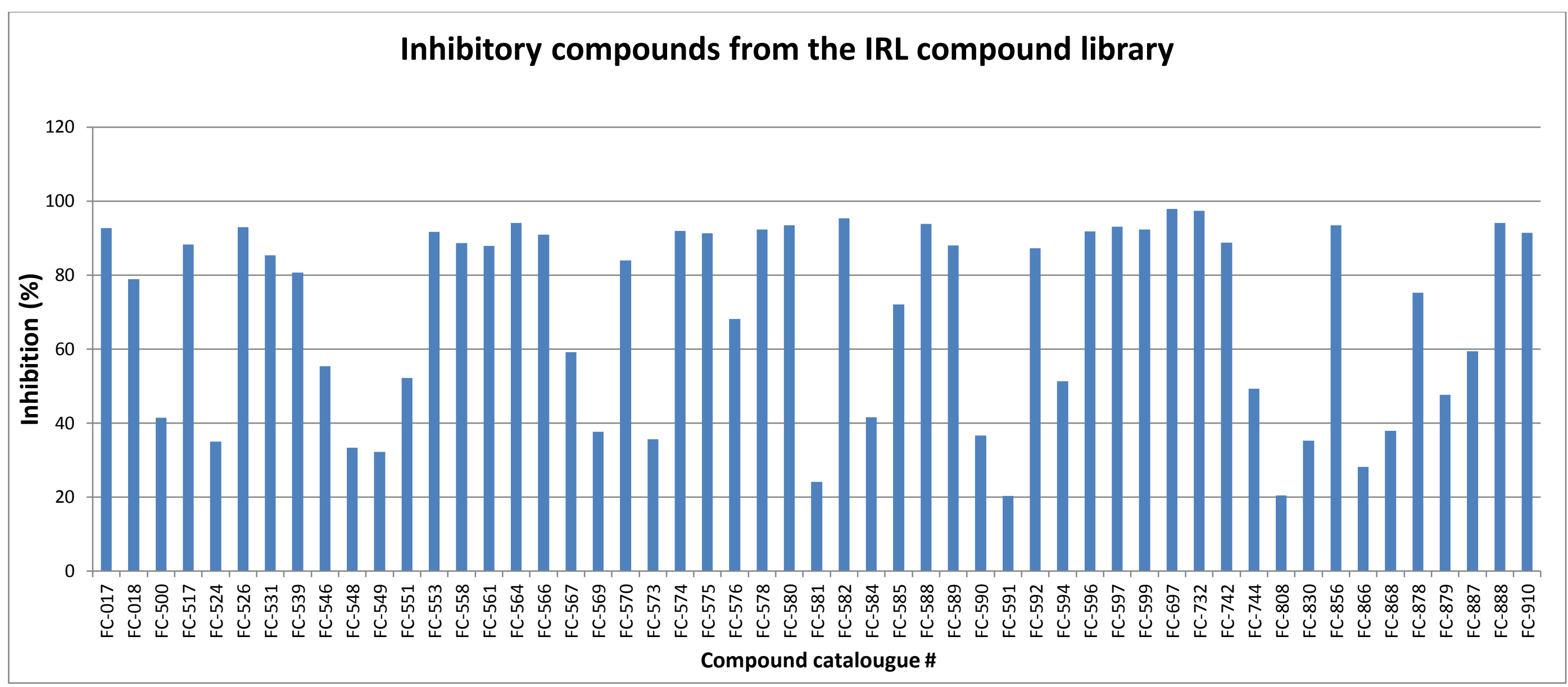

Figure 2.15. 53 compounds from the IRL library showed inhibitory activity of at least $20 \%$ in YCG326 in a liquid based inhibition assay. Compounds displaying inhibition >90\% are essentially completely inhibitory in this assay, with the remainder accounted for in background variation. This included all the compounds that were identified in the halo assay except for FC-571 and FC734. A significant number of compounds were identified as having complete or near complete inhibition despite not showing up at all on the halo assay. This intriguing result suggests a possible difference in drug uptake between liquid phase cultures and agar based media, which has clinical ADME implications. In addition these data demonstrate the increased sensitivity of a liquid based assay compared to a halo type solid phase assay. 


\subsubsection{Colony forming unit assay}

A colony forming unit assay was performed by treating YCG326 cells with high doses of FC-592 and FC-888 to determine if those compounds displayed cytotoxic or cytostatic activity. These were run alongside controls consisting of DMSO, cycloheximide treated cell (a known cytostatic compound) and rapamycin (a known cytotoxic compound) treated cells. Data from this assay would prove whether FC-592 and FC-888 killed cells outright (or caused irreversible growth arrest, which amounts to the same thing), or merely halted growth whilst in the presence of the compound.

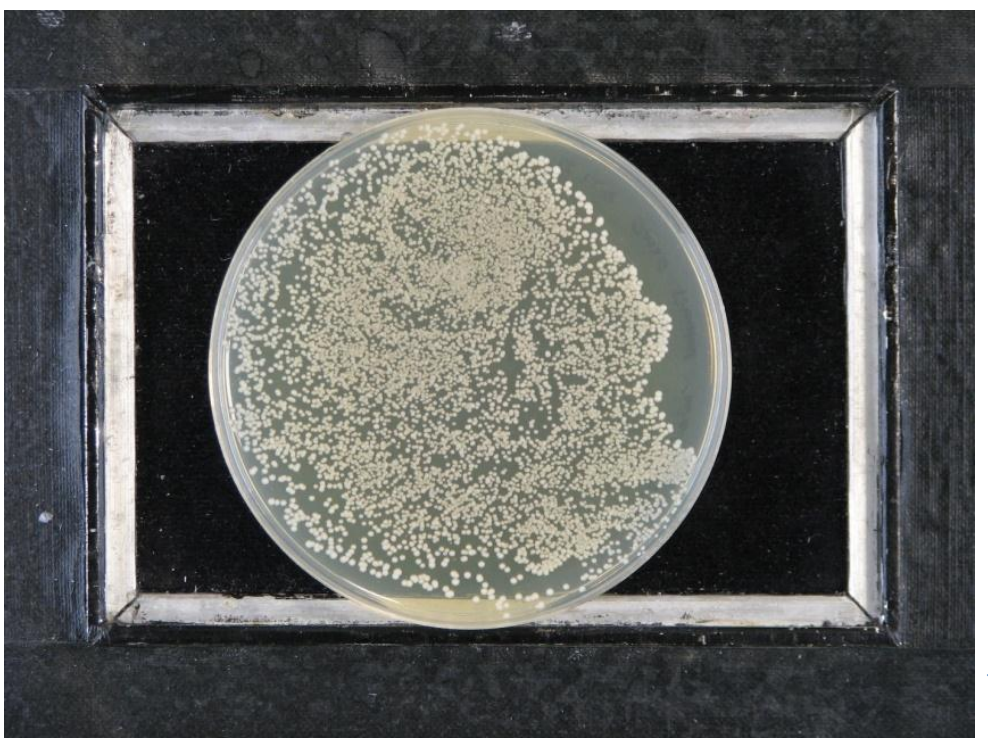

Figure 2.16 DMSO treated control shows good growth for colony forming unit assay. This result acts as a baseline for drug treatments.

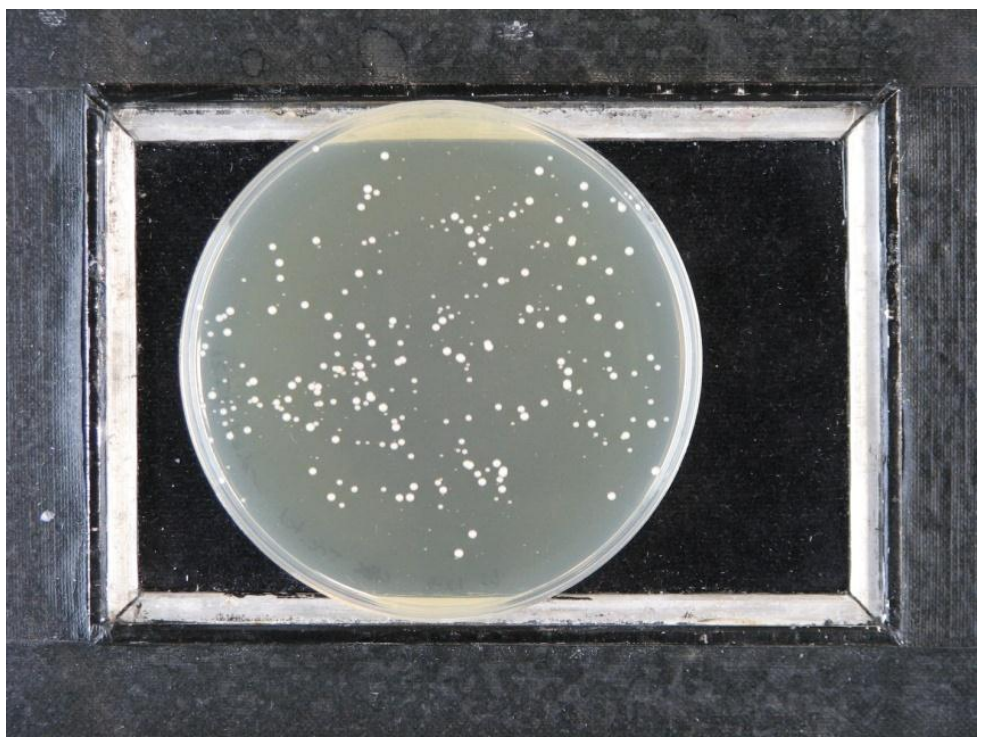

Figure 2.17 Cycloheximede treated control. Cells recovered from high dose $\mathrm{CHX}$ treatment, but showed a higher attrition rate than the DMSO treated control. Note the prevalence of petite colonies, a phenotype associated with the loss of mitochondria in the parent cells. 


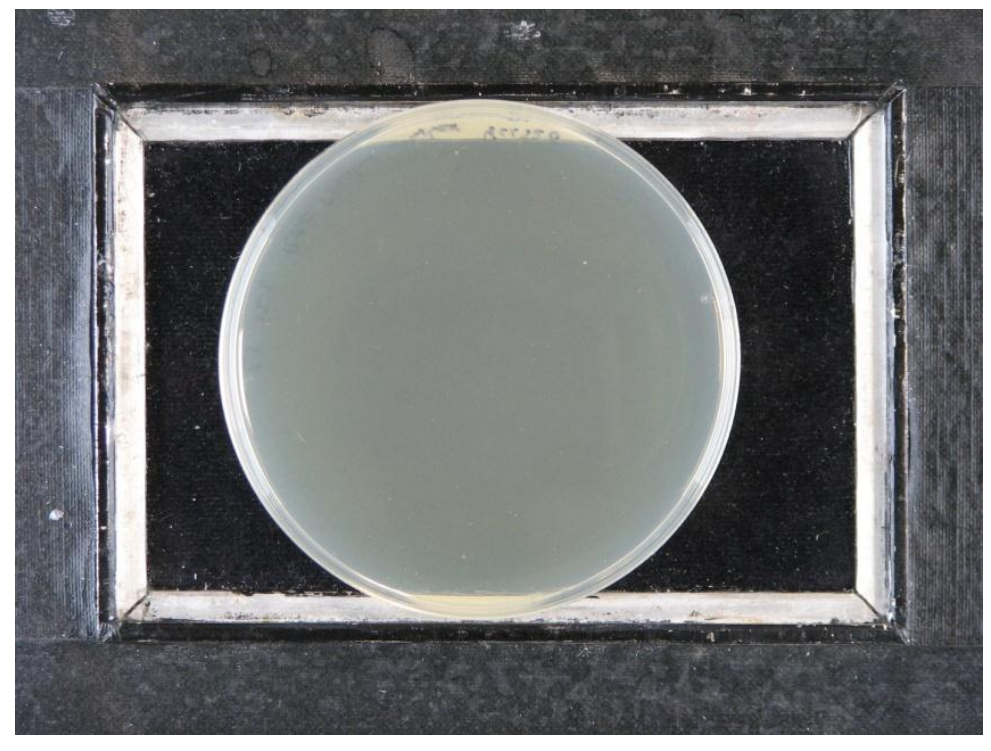

Figure 2.18 Rapamycin treated control. Cells treated with high dose RAP do not recover. Rapamycin irrevesably inhibits the TORC1 complex and serves as a model of a cytotoxic compound.

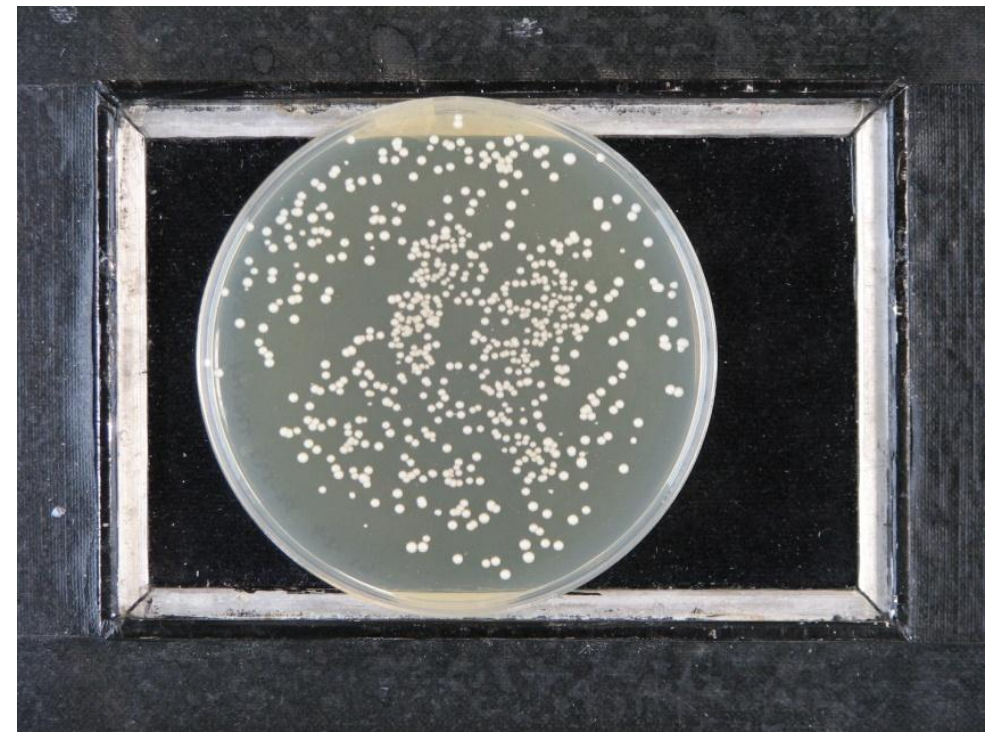

Figure 2.19 FC-592 treated cells recover after washing and growth on nutrient media. A lower proportion of cells survive compared to a DMSO treated control however, suggesting that cells are undergoing attrition, whilst not replenishing the population during FC-592 treatment. Few petite colonies are seen, in contrast with $\mathrm{CHX}$ treated cells. These data suggest that FC-592 has cytostatic activity.

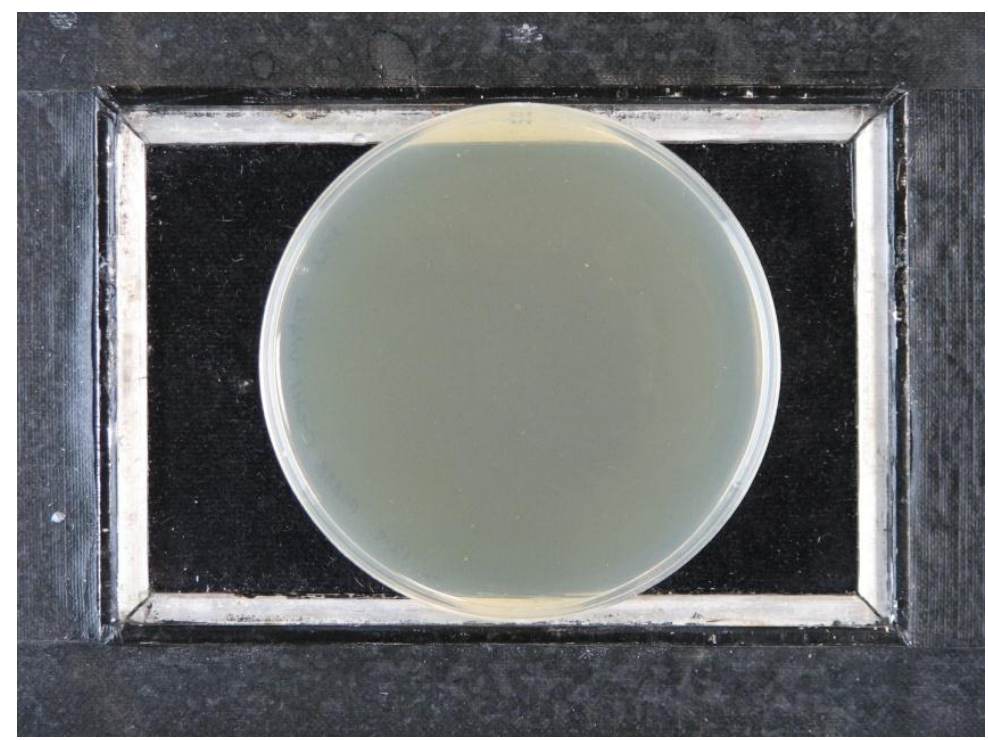

Figure $2.20 \mathrm{FC}-888$ treated cells do not recover after washing and growth on nutrient media. It is apparent that FC-888 displays cytotoxic activity, either irreversibly inhibiting or directly killing YCG326 cells. 
Results of the colony forming unit assay show that FC-592 displays cytostatic activity, whilst FC-888 is cytotoxic. This result has implications for both compounds. Chemotherapy of cancer, treatment of skin diseases and treatment of certain infections are conditions which are often treated with cytostatic drugs (Pervin et al. 2001). Cytostatic drugs can have a more specific mechanism that allows for increased target selectivity, for example of tumours (Chapes et al. 1988).

Cytotoxic agents such as FC-888 have several mechanisms, but tend to be broader in action (Valeriote \& Vanputten, 1975). Compounds may cause necrosis, in which cells lose membrane integrity and die rapidly as a result of cell lysis, or apoptotic pathways can be activated. Alternatively, the compound can cause irreversible inhibition of enzymatic activity, preventing cell proliferation and leading to eventual cell death (Poulin et al. 1992). 


\subsection{Discussion}

\subsubsection{Biologically active organic compound screening}

This project identified a range of biologically active small molecule organic compounds. The library of compounds obtained from Industrial Research Ltd. consisted of purely synthetic intermediates, by-products and enantiomers of organic synthesis work, and synthetic derivatives of natural products. This library was screened for inhibitory biological activity for mode of action studies in a yeast model system. 254 novel compounds were screened for inhibitory activity producing 22 'hits' for inhibitory activity on a 'halo' assay.

Several other compounds identified as inhibitory either possessed incomplete molecular mass data (meaning determining molar concentration was impossible), or proved too weak in a liquid phase dose response assay. These compounds were discarded from further study, although remain as a possible future project.

Traditionally, most drugs have either been natural products or synthetic derivatives of natural products. By 1990 , over $80 \%$ of drugs fell into one of these two categories (Li \& Vederas, 2009). Only in recent times have advances in chemistry lead to a focus on other sources such as synthetic diversity oriented libraries (Koehn \& Carter, 2005). In addition, this focus on synthetic sources has not lead to an increase in drug discovery (Gullo et al. 2006). This may be in part because of the complex structures of natural products with numerous oxygen-containing substituents and an abundance of centres of stereochemistry found in natural product derivatives (Butler, 2004).

This tendency is borne out by this project. Most of the hits found were synthetically modified diterpene natural products from New Zealand plant sources. These compounds include FC-561, FC566, FC-567, FC-570, FC-571, FC-574, FC-575, FC-578, FC-580, FC-582, FC-588, FC-589, FC-592, FC596 and FC-599. From a biology perspective, this makes sense as natural product compounds are presumed to have evolved for specific targets. 
Although it was decided not to expend resources characterising the mode of action of all the compounds identified as bioactive, information provided by Dr. Richard Furneaux suggested that compounds in the 500 series are likely to have similar structures, being derived from similar natural product compounds. This in turn means that compounds within this series are likely to have a similar mode-of-action. For this reason it was decided to only attempt to characterise one of the compounds in this series, FC-592. This compound was the most potent relative to the yeast strains tested of all the diterpene natural product derivatives, and was thus regarded as having the best chance for mode-of-action identification.

Of the purely synthetic compounds identified, the most potent was FC-888. This compound was also selected for further study due to its novelty and potency. Novel synthetics are not common amongst emerging anti-fungal agents (Di Santo, 2008), and it was felt that characterisation of the mode-ofaction of this compound would prove interesting.

\subsubsection{The halo assay as an inhibition screen in yeast}

The halo assay uses yeast cells seeded within nutrient agar to test for inhibitory activity in compounds applied to the surface of plates. Since small molecule compounds disperse evenly from their point of application, regions closer to this point will carry a higher dose of the compounds (Gassner et al. 2007). Yeast cells in these regions are inhibited in a dose dependant manner, meaning that comparatively more potent compounds will produce larger halos.

The halo assay in this project was effective in identifying inhibitory compounds, and did so in a way that is amenable to high throughput screening strategies. However, there were several issues with this method. Firstly, growing yeast within the agar produced gas bubbles which resulted in a series of split-like 'bubbles' within the medium. This problem was largely mitigated by switching from a HEPES based buffer to a MOPS based buffer, but remained an issue throughout the experiment. It is not known whether HEPES produces excess gas evolution, resulting in more 'bubbles', or produces a more brittle agar medium, causing more of the 'bubbles' to form given similar gas evolution. 

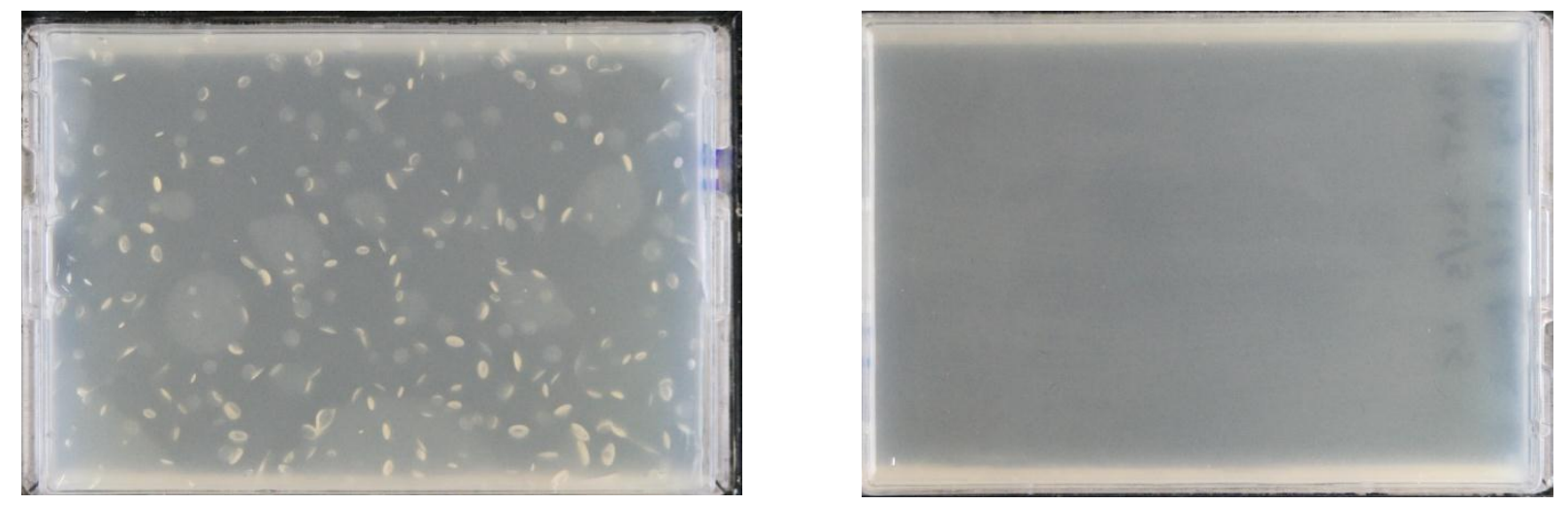

Figure 2.21 Halo assay test plates using $25 \mathrm{mM}$ HEPES (I) and $25 \mathrm{mM}$ MOPS ( $r$ ) to buffer media to physiological pH. A gas evolution in the HEPES plate caused a series of bubbles, which would have the potential to obscure any halos produced by the experiment.

Halo assay media takes a higher level of expertise to produce than an equivalent liquid based media.

This is because a live cell culture must be added at the correct concentration to molten agar media that is cool enough to not heat kill the cells. In addition, this cool media becomes more difficult to pour into plates. These factors should be kept in mind when instructing inexperienced researchers in this technique.

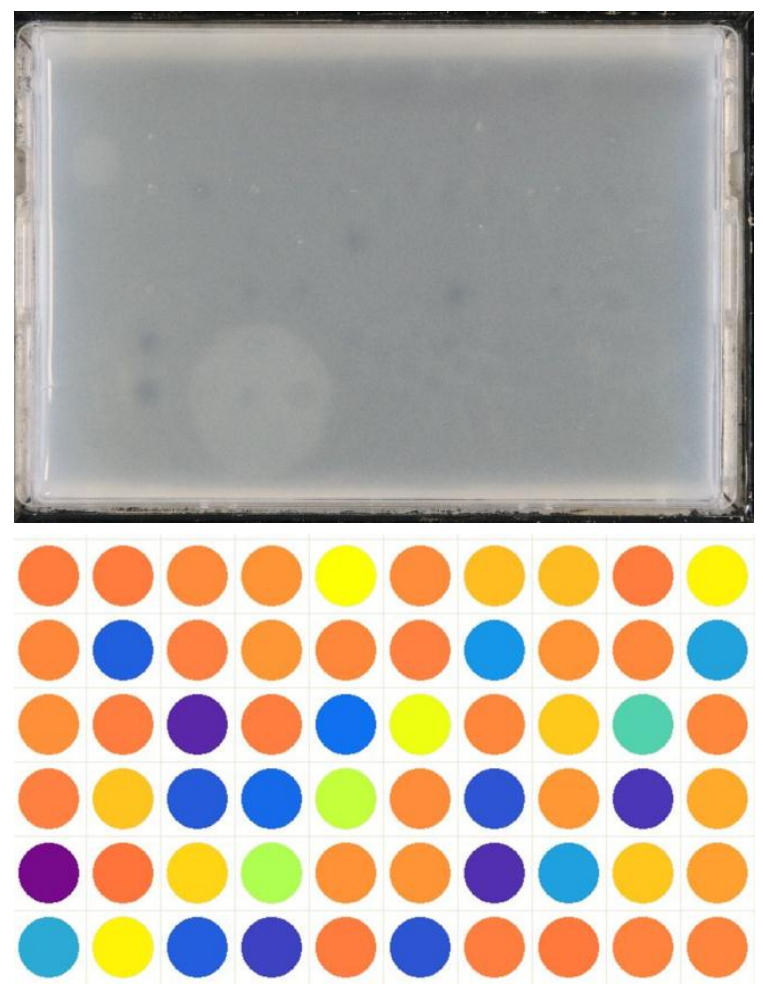

Whilst the halo assay performed well in identifying several inhibitory compounds, it proved to not be as sensitive as a liquid phase inhibition assay that was performed with the same compounds for comparison purposes. Where the halo assay

Figure 2.22 Comparison of inhibition assay results. Plate 2 from the halo assay (top), and a visual representation of output from the equivalent liquid phase inhibition assay plate (bottom). More blue circles represent a higher level of inhibition. Many more hits are seen in the liquid assay than in the solid phase halo assay.

identified 22 such compounds, the liquid phase comparison revealed 53 compounds which caused at least $20 \%$ inhibition in $\triangle P D R$ cells. In addition, quantitative aspects of inhibition (data about each compound's potency), had to be subjectively analysed in the halo assay (where the borders of the halos were often indistinct). Alternatively, a plate reading robot could be used to objectively measure inhibition levels in the liquid phase assay. 
This introduces less error into the measurement of results and would produce less variation between researchers or labs working in parallel of the same or similar compounds.

It should be noted that there were several compounds which were seen to potently inhibit yeast in the solid phase assay, but did not inhibit growth at all in liquid. The vice versa was also true. These results could be explained by differences in compound solubility or cellular uptake, and illustrate the differences in drug behaviour often seen between liquid phase and solid phase assays (Curran \& Bugeja, 2005).

\subsubsection{Dose response assays}

A series of dose response assays were carried out in differing conditions to determine the characteristics of the studied compounds with regards to general potency, $\mathrm{pH}$ conditions, the pleiotropic drug resistance (PDR) network, and solid phase vs. liquid phase assay type.

A liquid phase dose response assay showed that both FC-592 and FC-888 inhibited yeast growth in the low $\mu \mathrm{M}$ range. FC-592 inhibited all growth with a minimum inhibitory concentration of $11.21 \mu \mathrm{M}$ in the PDR attenuated ( $\triangle P D R$ ) strain YCG326, and at $~ 35 \mu \mathrm{M}$ in a wild type (WT) strain. FC-888 inhibited growth at $11.29 \mu \mathrm{M}$ in both $\triangle \mathrm{PDR}$ and WT strains.

The strong potency of both of these compounds has implications for further research. Although neither are being considered as lead compounds for medicinal research, they do display the potency required were such a direction to be undertaken. Because they are diluted in the bloodstream, and high molar concentrations of many compounds are associated with deleterious side effects (Bodor \& Buchwald, 2000), bioactivity must be achieved at a relatively low concentration. In addition, high drug potency is associated with lower risk of idiosyncratic drug reactions (Uetrecht, 2001; Uetrecht 2008).

Typical therapeutic drugs such as the commonly used antiepileptic phenytoin, which is given at a plasma concentration of $20-40 \mu \mathrm{M}$, operate in the low $\mu \mathrm{M}$ range (Thompson, 2000). If used in a 
hypothetical therapeutic setting, FC-592 and FC-888 would operate at a sub-inhibitory dose - i.e. likely in the high $\mathrm{nM}$ range. This means they are sufficiently potent to be considered for such an application if future research results justified it.

Strong potency is also a requirement for anti-microbials, another potential application for inhibitory compounds. As well as the need to completely inhibit microbial growth to avoid the rise of antibiotic resistance (Drusano, 2001), high molar concentrations of these agents can have pharmacokinetic issues such as renal complications (Pea et al. 2007). Therefore a low MIC is required in the consideration of such agents. The common antifungal ketoconazole has an MIC range of 1-2 $\mu \mathrm{M}$ in WT S. cerevisiae (Zhanel et al. 1998), on the same order of magnitude as FC-888. This makes antimicrobial applications a viable direction for research with this compound.

This project made use of a $\triangle P D R$ strain of yeast to increase sensitivity to the inhibitory compounds. This strategy proved effective in reducing the amount of compound required to conduct further assays. Conservation of compound became a key consideration in experimental design, as the available amount of each chemical was severely limited. $\triangle$ PDR cells were $\sim 3$ fold more sensitive to FC-592 relative to a WT equivalent allowing a greater amount of work to be done than would be possible with a WT strain.

A PDR dose response assay comparing the sensitivity of FC-592 in $\triangle$ PDR cells, WT cells, and a range of efflux pump single deletion mutants ( $\Delta \mathrm{yor} 1, \Delta \mathrm{snq} 2$ and $\Delta \mathrm{pdr} 5$ ), suggested that the compound was a substrate of the YOR1 plasma membrane ATP-binding cassette $(A B C)$ transporter. This efflux pump is known to mediate export of many compounds including oligomycin, amitrole and cycloheximide (Katzmann et al. 1995; Alamgir et al. 2010), many of which are organic anions (Cui et al. 1996). This suggests the possibility that FC-592 may be deprotonated; although this doesn't reveal much about the target or mode-of-action of the compound as these PDR efflux pumps are known to mediate the transport of a wide range of unrelated substrates (Balzi \& Goffeau, 1995). 
On the other hand, FC-888 proved not to be a substrate for the drug efflux pumps mediated by the PDR network, with no differences in sensitivity observed between PDR attenuated strains and PDR competent strains. The high potency of FC-888 even in WT cells however, meant that sufficient quantities of the compound were available to complete all necessary work.

The evidence that FC-592 is a substrate of the membrane ABC transporter Yor1p is an indication that it might reach the cytosol in an anionic or deprotonated state. This is because other compounds whose efflux has been shown to be mediated by this protein exist in anionic form (Cui et al. 1996). Supposing anionic dependant activity, one might therefore expect that at lower $\mathrm{pH}$ conditions, where protonation is driven, FC-592 would show ablated activity. However, this is not seen in a dose response assay conducted in varying $\mathrm{pH}$ conditions. Assays at $\sim \mathrm{pH} 4.0$ and $\sim \mathrm{pH} 7.0$ show identical inhibition profiles. Therefore, either the compound is not protonated even at low pH conditions, or its activity is not ablated by protonation. Because the mechanism by which PDR substrates are bound and effluxed from the cell is largely unknown (Balzi \& Goffeau, 1995), there exists the strong possibility that Yor1p does not require an anionic substrate, and that FC-592 does not exist in a deprotonated form. However, there is no evidence in the literature to support this determination about Yor1p.

Additionally, the $\mathrm{pH}$ dependant dose response assay showed that FC-888 was not affected by $\mathrm{pH}$ conditions either. This is expected for this compound however: a halogenated aliphatic ester, this compound has no hydrogens which are easily dissociated from it. Therefore it is unlikely to be affected by changing $\mathrm{pH}$ conditions.

A solid phase dose response was conducted with FC-592 and FC-888 for comparison to liquid phase results. FC-592 was determined to have a $\sim 3$ fold higher MIC in a solid phase agar based assay. As previously mentioned, these differences could be due to differences in solubility of the compound in different media types or mechanism of cellular uptake. A more remote possibility is differences in metabolism between the solid phase interface and liquid culture. Microorganisms are known to 
behave differently on a solid surface and in a liquid culture environment (ZoBell, 1943; Stotzky \& Rem, 1967). Therefore differing metabolic activity could affect the concentration at which FC-592 inhibits yeast growth.

FC-888 was completely inactive at the concentration ranges tested in solid phase dose response assays. This is despite the fact that the compound displayed a potent inhibitory effect on the halo assay. Because the compound inhibited yeast growth on the agar based halo assay, it is unlikely that this reduction in inhibitory activity is the result of differences in solubility or cellular uptake. Instead it is proposed that the compound was degraded by the $\sim 50^{\circ} \mathrm{C}$ temperature to which it was subjected during plate production. The compound was applied to liquid SC agar during the making of plates for this assay, and could have been affected by this temperature.

\subsubsection{Colony forming unit assay}

An assay was conducted to determine whether FC-592 and FC-888 permanently killed cells (cytotoxic activity), or temporarily inhibited their growth (cytostatic activity). FC-592 treated cells managed to recover and grow on nutrient media after washing, although there were less colony forming units than in a DMSO treated control. This is likely because the cell population undergoes natural attrition whilst under compound treatment. Even though the compound does not directly kill the cells, because it stops the population replenishing itself, there are a lower proportion of viable cells after treatment relative to the control.

Medicinally, cytostatic agents have proven very beneficial in fighting tumours with their ability to induce cell growth arrest (Chapes et al. 1988). Due largely to the multiple mutations they possess, cancer cells have very high death rates (New Zealand Institute of Chemistry, 2012). Thus, preventing reproduction leads to a reduction in tumour size. These drugs gain their anti-tumour specificity from the higher attrition rate found in these cell types 
FC-888 treated cells did not recover in this assay, indicating that they were either directly killed or irreversibly inhibited by the treatment, and thus this compound exhibited cytotoxic activity. Cytotoxic agents function in a myriad of ways, including alkylation of DNA (New Zealand Institute of Chemistry, 2012) and induction of apoptosis (Wang et al. 2000). Whilst many cytotoxic agents have utility as chemotherapeutics treating non-infectious diseases (Fred Hutchinson Cancer Research Center, 2012), a primary use for them is as anti-microbial agents (Talbot et al. 2006). This is additional evidence that anti-microbial activity could be a potential application for a compound like FC-888.

\subsubsection{The chemistry of FC-592 and FC-888}

It is possible to glean some insight into the characteristics of novel compounds based on their chemical structure and predictions about their chemistry. There are several determinations that can be made about FC-592 and FC-888 in this manner.

FC-592 ((2R,3R,4aR)-4-butyl-3-(hydroxymethyl)-4a,8,8-trimethyl-decalin-2-ol) is a non-polar saturated bicyclic alcohol. Therefore it is a fairly stable compound for which little obvious chemistry suggests itself. As previously mentioned, the compound's apparent affinity for the plasma membrane $A B C$ transporter Yor1p suggests that it might exist in an anionic state within the cytosol. A possible mechanism for such a reaction is:
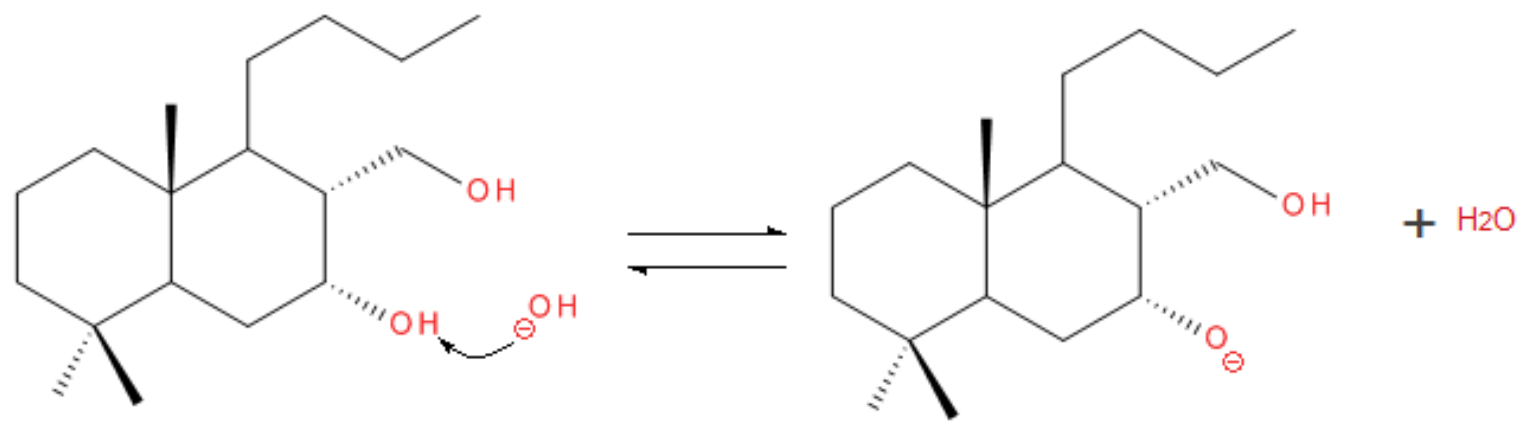

Figure 2.23 Lewis diagram anion formation. Although unlikely, it is possible that deprotonation could proceed on the basis of hydrogen bonding in the oxyanion product lowering the alcohol pKa. However, this is unlikely to have a realistic pKa value (Paul Teesdale-Spittle, personal communication), unlikely to occur below pH 12. 
The anion product seems very unlikely in a cytosolic context because the pKa for proton dissociation likely requires highly basic conditions. Since the compound has proven to be similarly active in $\sim \mathrm{pH}$ 4.0 and $\sim \mathrm{pH} 7.0$, this possibility can effectively be discounted. Therefore it is more likely that Yor1p in this case is mediating the efflux of a non-ionic molecule. Although Yor1p (Cui et al. 1996) has been associated with anion efflux, some substrates for the transporter, including cycloheximide (Dudley et al. 2005) and amitrole (Alamgir et al. 2010), are unlikely to be anionic in cytosolic conditions. Because of this the most likely explanation is that Yor1p more commonly mediates the efflux of nonanionic species than is suggested in the literature.

The compound is a highly lipophilic molecule with a polar diol region, that is likely to be a nonspecific binder to a range of hydrophobic pockets and environments, which might include the cell membrane or hydrophobic pockets within enzymes. Drugs with lipophilic properties are more easily incorporated into the phospholipid bilayer membrane (Schwendener \& Schott, 2010), meaning they can diffuse across membranes without the need for pharmacokinetic strategies such as liposome incorporation. In addition, hydrophobic drugs are likely to have a high lipophilic efficiency (LiPE) score, which is associated with 'druglikeness' (Leeson \& Springthorpe, 2007). LiPE is a statistical measure which combines metrics of the drug's potency and lipophilicity (Edwards \& Price, 2010). Quality drug candidates have a high LiPE, combining permeability through biological membranes and a reduced chance of idiosyncratic drug reactions and off-target toxicity (Uetrecht, 2001; Uetrecht, 2008; Hughes et al. 2008).

Some lipophilic antimicrobials such as the polyene macrolides are known to disrupt cell membranes (Bolard, 1986), leading to cell death by a number of mechanisms such as pore formation resulting in $\mathrm{K}^{+}$leakage and the peroxidation of membrane lipids. However it is clear that FC-592 does not result in cell death, as it is a cytostatic compound rather than a cytotoxic one.

In contrast, $n-3$ and n-6 polyunsaturated fatty acids have been shown (Diggle et al. 2000) to cause reversible antiproliferative and irreversible cytostatic effects, possibly due to lipophilic albumin- 
complexed fatty acid metabolites rapidly incorporated into normal cell membranes which are predicted to alter the functioning of proteins within cell membranes - causing cytostasis (Shires et al. 1989). Whilst there is no evidence that FC-592 has anything in common with these mechanisms, the several properties that they have in common (lipophilicity, cytostatic activity) makes it an intriguing direction for future research, which could include assays into cell membrane disrupting agents such as a $\beta$-galactosidase leakage assay (O'Niel et al. 2004).

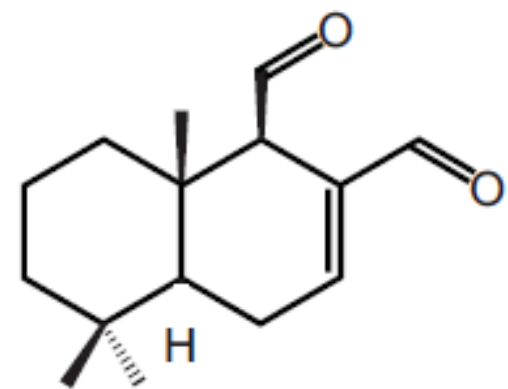

Polygodial

Figure 2.24 Polygodial, a drimane sequiterpene found in a range of peppery shrubs. Source: Escalera et al 2008.

FC-592 bears some structural similarity to drimane sesquiterpenes. These compounds are members of the deterrent natural product terpene family which are characterised by reactive unsaturated dialdehyde moieties (Gershenzon \& Dudareva, 2008), and are found in various plants, fungi, animals and marine invertebrates (Iken et al. 1998). FC-592 lacks the aldehyde groups which are implicated in the mode-of-action for these compounds through activation of TPRA1, an ion channel involved in inflammatory pain signalling initiated through influx of extracellular $\mathrm{Ca}^{2+}$ into the cells (Escalera et al. 2008), and is therefore unlikely to act in the same manner. However, drimane sesquiterpene compounds are inactive absent the dialdehyde moiety (Szallasi et al. 1998), including lacking inhibitory activity at relatively high cytosolic concentration ( $200 \mu \mathrm{M}$, Escalera et al. 2008). Therefore it is possible to conclude that the basic structure does not initiate a particular inhibitory cellular response. The activity of FC-592 is more likely to be mediated by its putative hydrophobic pocket binding properties.

FC-888 (ethyl 3-bromoprop-2-ynoate) is a halogenated aliphatic alkyne ester. The bromine atom attached to the triple bonded carbon is a strong leaving group, meaning that the compound is highly susceptible to nucleophilic attack: 
1.

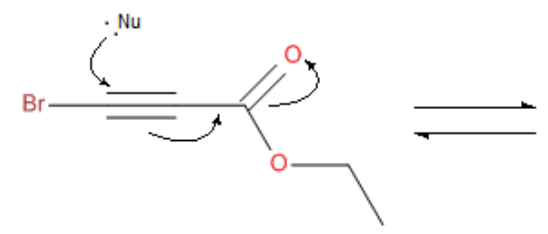

2.

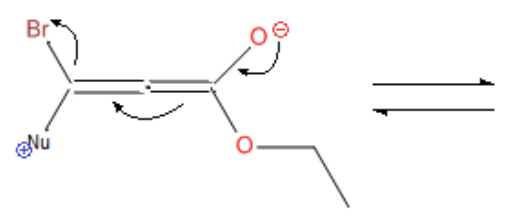

3.

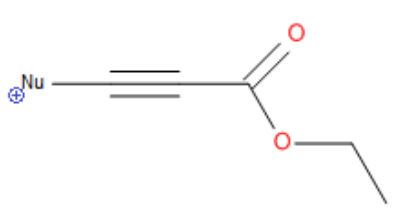

Figure 2.25 Nucleophilic substitution of bromine with a cellular nucleophile. The highly reactive triple bond and the strong $\mathrm{Br}$ leaving group make this an energetically favoured reaction. In this scenario, FC-888 would bind to multiple targets and affect many processes throughout the cell, including DNA transcription and replication. .

The substitution reaction proposed in figure 2.25 would see cellular nucleophiles such as DNA or certain amino acids bound to FC-888. The nucleophile attacks the electrophilic triple-bond (1), which is stabilized as an intermediate by HOMO-LUMO interaction with the carbonyl oxygen (2). Bromine leaves this unstable intermediate as a bromide ion, creating an overall stabilized organic cation (3).

This reaction could have a range of deleterious effects including altering the structure of proteins, distorting the shape of nucleic acid chains, and interfering with the replication of DNA. However, further reactions are likely that could have bearing on the mechanism of the compound in a cellular context. Further reaction of product (3), still containing a highly reactive triple bond, is expected to be thermodynamically favourable, and could see the reaction proceed along several pathways:

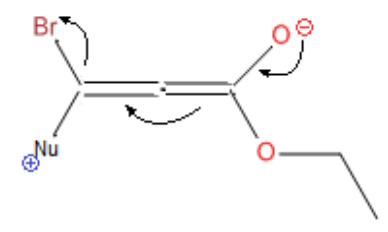

5.<smiles>I[I-]</smiles>

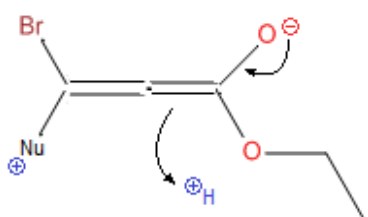

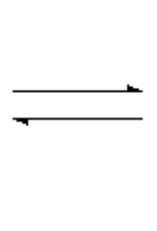

6.

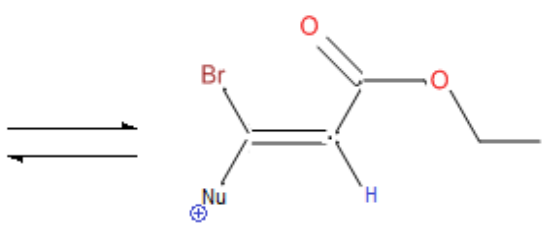

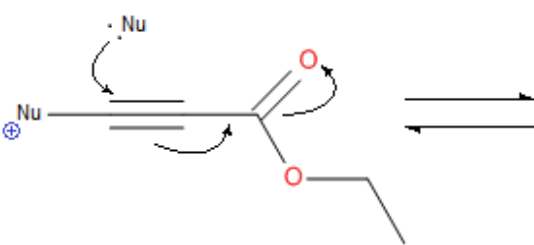
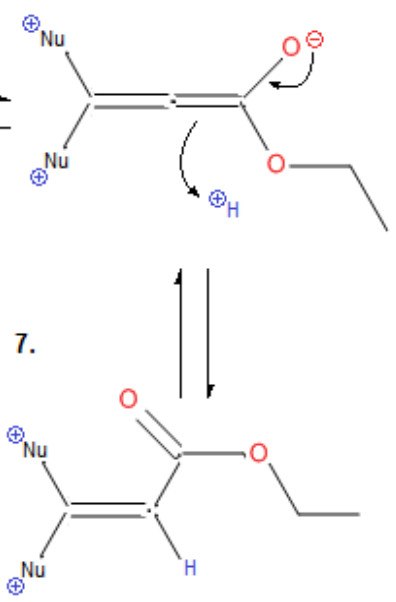

Figure $\mathbf{2 . 2 6}$ Nucleophilic substitution of FC-888 is completed. Unstable intermediates 4 \& $\mathbf{5}$ undergo protonation to achieve the proposed stable final products $(6 \& 7) .7$ is the energetically favoured product, whilst 6 is the sterically favoured product. Still prone to conjugate addition/elimination, 6 is an unlikely end product. 
In these reactions, more stable products are created than (3). The unstable intermediates (4) \& (5) are protonated resulting in an alkene ester. In (6), the bromine remains as a substituent. The likelihood of conjugate addition/elimination makes this an unstable intermediate as well, making (7) the energetically favoured product. The Nu groups are likely to be large macromolecules such as proteins or nucleic acids. The formation of (7) could possibly be sterically hindered, driving the equilibrium reaction back towards (6). However, alkylating agents such as cis-platin (Rosenber et al. 1965) have proven to have potent DNA cross-linking properties (Thompson, 2007), showing the suggested mechanism for the formation of (7) is possible and has an empirical basis.

This proposed mechanism suggests a great deal about the likely mode-of-action of the compound in inhibiting yeast growth. The primary mechanism of toxicity that suggests itself is DNA alkylation, which is compatible with the recorded activity of the related compound propyl hept-2-ynoate (PubChem, 2012). Soluble, electrophilic alkylating agents are highly toxic because of their ability to alkylate DNA, disrupting normal genetic function (Stefanidakis \& Gwyn, 1993). Alkylated DNA either does not coil/uncoil properly, or cannot be processed by information-decoding enzymes.

Bifunctional alkylation agents can form cross-links between the complementary strands (Tomasz et al. 1988), causing selective inhibition of DNA replication. For this reason, alkylating agents such as mitomycin C have been used to target rapidly dividing cells such as tumours (Tomasz, 1995), as they are more heavily affected than more slowly dividing, healthly, somatic cells. However, alkylating agents often have mutagenic effects (Schloacher et al. 2006), including carcinogenic mutations, resulting in higher incidence of cancer after exposure (Lawley, 1980). Assuming formation of product (7) above is favourable, FC-888 would be an example of a bifunctional alkylation agent, and could be explored for similar utility.

Alkylating agents can target a broad array of cellular processes, and so may lack specificity desired in a therapeutic. In S. cerevisiae low levels of the alkylating agent methyl methanesulfonate have been shown to cause significant gene transcript increases in no less than 325 genes (Jelinsky \& Samson, 
1999), with decreases in a further 75 . This illustrates the extensive range of effects that cellular alkylation can have, with affected genes ranging from DNA repair to heat shock to protein kinases. It should therefore be acknowledged that not all alkylating agents have the required specificity for pursuit as therapeutics. Most known therapeutic cross linking agents are large and complex enough to recognise 2-3 nucleotides as binding sites (Bose et al. 1992) or are active only in hypoxic conditions (Patterson et al. 2007). It is unlikely that FC-888 has sufficient structural complexity to mediate specific inhibition in this manner. However there are a few examples such as cis-platin that are structurally simple but useful therapeutically (Thompson, 2007). 


\section{Chemigenomic Analysis}

\subsection{Introduction}

\subsubsection{Gene networks}

Genes typically do not work in isolation, but work with each other in complex pathways and networks (Awad et al. 2012). Understanding how drugs operate as part of these networks, for example binding certain gene products to express a desired phenotype, is important to our ability to optimise and develop drugs into therapeutically useful treatments. Inherent in this understanding is dissecting complex sets of interactions between small molecule probes and a diverse set of genes and gene products, which in turn interact with other genes and gene products to create further downstream effects.

The use of S. cerevisiae as a model allows an ingenious method of characterising the mechanism of drug candidate interaction with genetic networks, known as chemical genomic analysis (Zhao et al. 2005; Singh-Babak et al. 2012).

\subsubsection{The deletion mutant array}

Per Boone et al. (2007), mutations in most eukaryotic genes have little distinguishable effect. Only $\sim 20 \%$ of genes appear to be essential for viability when individually deleted in haploid S. cerevisiae cells grown under normal conditions. This is evidence that the vast majority of genes do not work on their own to achieve their intended function, but as part of a robust and redundant genetic circuit

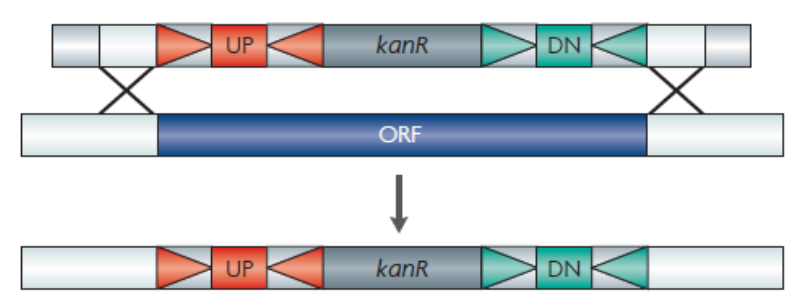

Figure 3.1 Gene deletion strains are created by PCR mediated mediated gene disruption strategy that exploits the high rate of homologous recombination in yeast. A kanR marker disrupts the gene of interest, and is flanked by unique $20 \mathrm{bp}$ oligomer barcode. Reprinted by permission from Macmillan Publishers Ltd: Nature Reviews Genetics, Boone et al. 2007. which resists a loss of function at any one node. Indeed, this result illustrates the tendency of organisms to build buffering

schemes that allow phenotypic stability despite genetic variation, environmental 
changes and random events such as mutations (Hartman et al. 2001).

These two phenomena, interconnected gene networks and robust genotypes that can resist loss of function mutations in most genes, allow for an elegant tool to parse genetic interactions. Given that null mutants are usually viable, researchers have built a library of such knockout mutants which encompasses the majority of the genome (Winzeler et al. 1999). Strains are constructed via PCR mediated disruption of individual ORFs with a kanamycin resistance marker flanked by a unique 20 bp oligomer identifying barcode region. These libraries, which exist in haploid as well as homozygous and heterozygous diploid forms (Giaver et al. 2003), have become valuable tools for research into the function of gene pathways and regulatory networks (Zhao et al. 2005), disease systems (Munkacsi et al. 2012) and drug mechanism (Parsons et al. 2006).

These feats are possible because whilst a knockout mutant for a non-essential gene may be viable, it is weakened in the specific pathway or network within which that gene is associated. A second deletion in the same pathway or a related process could cause an enhanced phenotype, which is readily detectable as a synthetic sick or synthetic lethal growth type (Tong et al. 2001). By mass mating a comprehensive gene deletion library such as the Boone Array (Tong \& Boone, 2007, known as the Deletion Mutant Array or DMA) in ordered arrays with a second null mutant, double mutants displaying an enhanced growth defect can be identified. In this way, interactions between genes can be identified and a picture of pathways and networks can be built. 

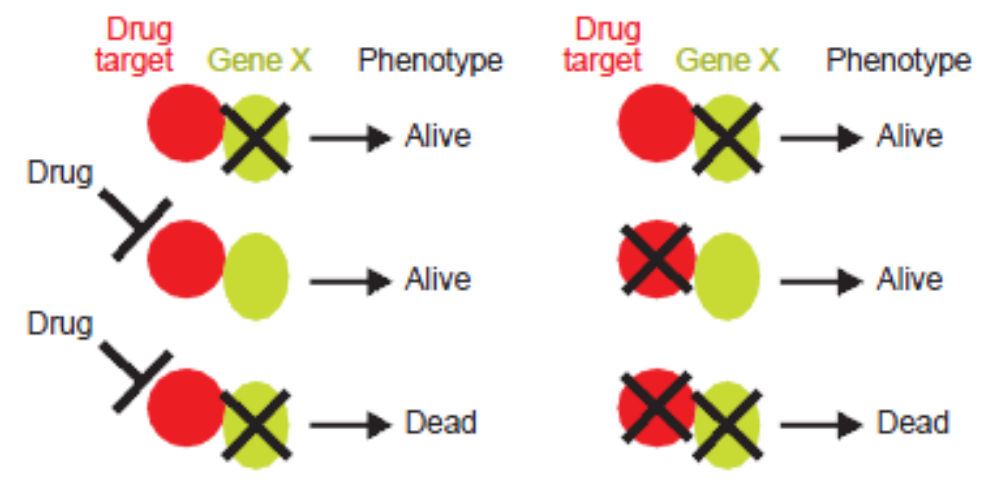

In an analogous manner, gene deletion libraries can be used (Nislow \& Giaever, 2007) to probe the mechanism of small molecule

Figure 3.2 The DMA can be used to identify drug targets. Whilst the cell is viable when either the drug target or related gene is disrupted, it is incapable of surviving the double insult. By noting the pattern of phenotype enhancement in a chemical genomic array experiment, target pathways of individual drugs can be identified. Reprinted by permission from Macmillan Publishers Ltd: Nature Biotechnology, Parsons et al. 2004.

drugs. Gene knockout strains

from an ordered library such

as the Boone Array are assayed for sensitivity to the compound of interest. Whilst each genetic network is typically able to compensate for a single gene deletion, if the network comes under insult from a chemical compound as well, it can often not recover. In this way, cells which are deficient in genes in a certain network are more vulnerable to chemical attack on that network. By analysing patterns of synthetic sick or synthetic lethal cell growth, the target pathways of the compound can be identified. Assays conducted using a heterozygous deletion library (where each strain has one functional copy of a gene) are known as Haploinsufficiency Profiling (HIP) assays, whilst homozygous based assays are known as Homozygous Profiling (HOP) assays.

\subsubsection{Barcode microarrays}

A key limitation of this approach is the quantity of compound used in such a chemigenomic

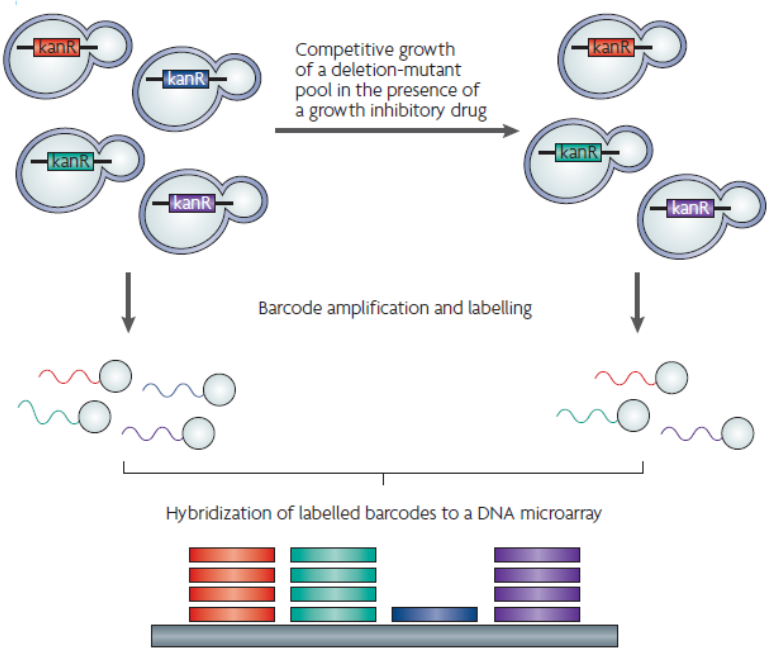
array experiment. Typically the DMA is pinned Figure 3.3 Parallel analysis of large pools of deletion mutants allow populations of pooled mutant cells to be screened for sensitivity in a drug efficient manner. Pooled onto agar plates containing a minimally cells are grown in the presence or absence of a growth inhibitory drug. Genomic DNA is extracted from the pool, inhibitory level of the drug. In the case of barcodes representing each individual strain are PCR amplified and stained with a fluorescent dye, and hybridized to a microarray chip. Drug-sensitive mutants are identified by a low signal for that barcode compared to an untreated control. Reprinted by permission from Macmillan Publishers Ltd: Nature Reviews Genetics, Boone et al. 2007. 
scarce novel compounds however, this can be prohibitive.

The development of yeast deletion libraries with identifying DNA barcodes allows a simple and elegant method conducting these experiments (Ho et al. 2009). Rather than conducting separate assays for each of $\sim 6000$ deletion mutants, it is possible to pool the mutants and treat them together with minimally inhibitory concentrations of the selected drugs. The inhibition profile of each mutant within the pool is determined by extracting the genomic DNA and hybridizing to a barcoded microarray chip. This is typically done alongside an untreated control, using competitive hybridization (Giaever et al. 2002). Genes of greater relative importance for a drug condition diminish faster relative to those with no import in the presence of that drug, and the hybridized tag signal for that strain is lower on the micro array chip. Therefore, those genes required for growth under a certain condition can be identified and ranked in order of their relative contribution to fitness in a single experiment.

\subsubsection{Glycosylation biosynthetic processes and the unfolded protein response}

Glycosylation is the reaction in which a glycosyl donor (carbohydrate), is enzymatically joined to a hydroxyl or other functional group of a glycosyl acceptor, typically proteins, lipids, or other organic molecules (Drickamer and Taylor, 2006). Glycosylation biosynthetic processes are important for cotranslational and post-translational modification of cellular products, serving a variety of structural and functional roles in membrane and secreted proteins.

During this project evidence suggested that one of the studied compounds, FC-592, may disrupt glycosylation biosynthetic processes and processes linked to the secretory pathway. A possible consequence of this disruption could be the mis-folding of proteins in the endoplacmic reticulum (ER), a process that could lead to activation of the unfolded protein response.

The unfolded protein response (UPR) is a cellular stress response that is conserved between many eukaryotic organisms, including yeast and mammals (Bernales et al. 2006). The UPR is activated in 
response to an accumulation of unfolded or misfolded proteins in the lumen of the ER, where a series of reactions restores normal cell function by halting protein translation and upregulating signalling pathways controlling chaperones involved in protein folding (Okamuraa et al. 2000) and the induction of ER-associated protein degradation, or ERAD (Meusser et al. 2005). The transcription factor Hac1p is activated by the threonine-kinase transmembrane mis-folded protein sensor Ire1p, which splices a translation inhibiting intron from HAC1 (Jonikas et al. 2009; Bernales et al. 2006). Hac1p transcriptionally upregulates a series of chaperones such as Kar2p, PDI1 and FKB2p (Casagrande et al. 2000), which restore proper ER function. A Hac1p-responsive promoter driving green fluorescent protein (GFP) expression can be used as a reporter to indicate activation of the UPR. Visualisation of Hac1p driven GFP expression is achieved via confocal microscopy (Peter Bircham, personal communication; Bircham et al. 2011).

Glycosylation stabilises folding of proteins during and soon after synthesis and its disruption often results in activation of the UPR. Thus activation of the UPR is a possible method to confirm disruption of glycosylation biosynthetic processes. A negative result does not rule out an effect on glycosylation however, as levels of protein misfolding may be too low to activate the UPR. Other glycosylation targets are also possible, as the process is also present in the cytoplasm and nucleus as the O-GIcNAc modification (Zachara et al. 2004), among others.

\subsection{Aims and objectives}

The aim of this chapter is to determine chemigenomic inhibition profiles for the selected novel compounds FC-592 and FC-888, and to characterise a target gene or pathway. The key objectives are:

1. Perform a chemigenomic analysis experiment using barcode microarray techniques with FC592 and FC-888 
2. Confirm sensitive mutant profiles through dose response

3. Use analytic techniques to establish a likely target for each compound

\subsection{Methods and materials}

\subsubsection{Yeast strains}

\begin{tabular}{|c|c|}
\hline Strain & Genotype \\
\hline Y7092 & 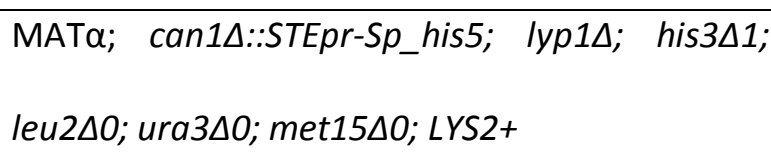 \\
\hline YCG326 & 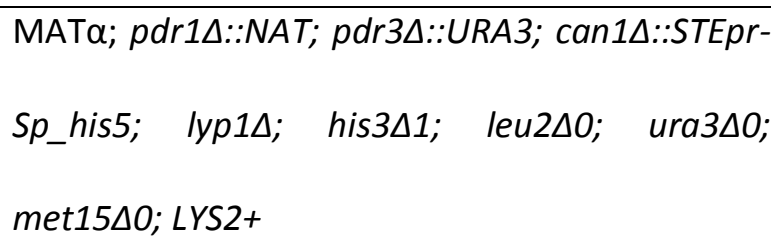 \\
\hline $\begin{array}{l}\text { Boone Array Pooled Homozygous array (HOP } \\
\text { pool; Winzeler et al. 1999) }\end{array}$ & 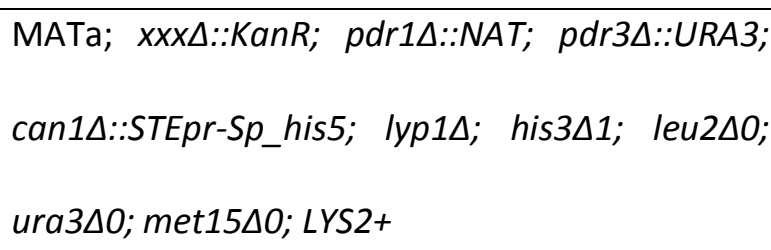 \\
\hline $\begin{array}{l}\text { Individual Boone array single deletion mutants } \\
\text { designated as } x x x \Delta\end{array}$ & 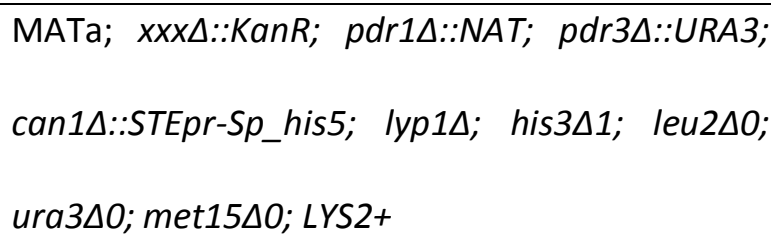 \\
\hline YCG266 - 4xUPRE-GFP & 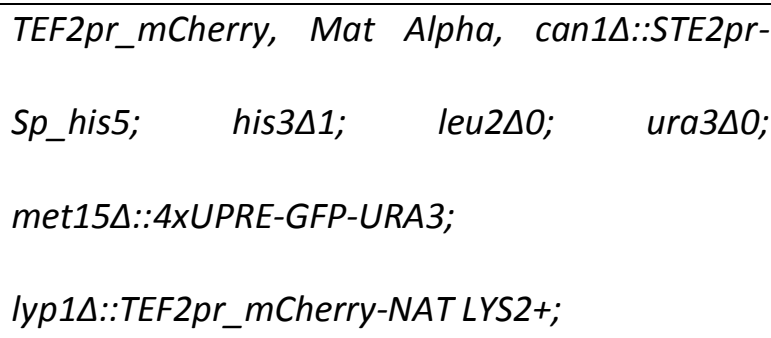 \\
\hline
\end{tabular}

\subsubsection{Growth media}

Yeast growth assays were performed in the following media formulations. All media was made up with distilled deionised $\mathrm{H}_{2} \mathrm{O}\left(\mathrm{ddH}_{2} \mathrm{O}\right)$ and autoclaved at $120^{\circ} \mathrm{C}$ for $20 \mathrm{~min}$. Sterile glucose was added to media post autoclave: 
Yeast peptone dextrose (YPD): As in Chapter 2.

Synthetic complete (SC): As in Chapter 2.

Synthetic deficient (SD) - Ura: As in Chapter 2.

NAT: As in Chapter 2.

G418: As in Chapter 2.

\subsubsection{Compounds and chemicals}

General chemicals and materials used include: $100 \%$ methanol, ethanol (absolute), ethanol (96\%), Dimethyl Sulfoxide (DMSO), MasterPure Yeast DNA purification kit (Epicenter Biotechnologies), and TE buffer (Tris $89 \mathrm{mM}$, boric acid $89 \mathrm{mM}$, EDTA $2 \mathrm{mM}, \mathrm{pH} 8.3$ ). PCR reagents used include: 10x Platinum Taq buffer (Invitrogen), $\mathrm{MgCl}(50 \mathrm{mM})$, dNTPs (5 mM each) and Platinum Taq (5 units/ $\mu \mathrm{L}$, Invitrogen). Primers used consisted of the following:

\section{PCR Primers:}

1. D1

2. U1

3. D2comp-Cy3 or -Cy5

4. U2comp-Cy3 or -Cy5

Blocking primers:

5. D2block

6. U2block
5'-CGGTGTCGGTCTCGTAG

5'-GATGTCCACGAGGTCTCT

5'-Cy [3/5]-CGAGCTCGAATTCATCGAT

5'-Cy [3/5]-GTCGACCTGCAGCGTACG

FC-592 and FC-888 were obtained from Dr. Richard Furneaux of Industrial Research Ltd. (Gracefield, New Zealand). These compounds were dissolved to working concentrations in DMSO. Dithiothreitol (DTT), a UPR inducer, was obtained from SigmaAldrich, and dissolved in $\mathrm{ddH}_{2} \mathrm{O}$. 


\subsubsection{Determination of $\mathrm{IC}_{30}$ concentrations}

Dose response assays were conducted for FC-592 and FC-888 to determine 30\% Inhibitory Concentration $\left(\mathrm{IC}_{30}\right)$ concentrations. This is the concentration at which $30 \%$ of growth is inhibited compared to an untreated control. A $500 \mu \mathrm{L}$ aliquot of the Homozygous Profiling (HOP) pool was seeded into $\sim 50 \mathrm{~mL} \mathrm{SC} \mathrm{G418} \mathrm{media} \mathrm{and} \mathrm{grown} \mathrm{at} 30^{\circ} \mathrm{C}$ overnight in a shaking incubator. Cell titre was determined via haemocytometer and diluted to $10^{5} \mathrm{cell} \mathrm{mL}^{-1}$ with fresh SC media. $10 \mathrm{~mL}$ aliquots of this cell suspension were dispensed into $50 \mathrm{~mL}$ Falcon tubes. Serial dilutions of each compound were performed in DMSO in 96-well reagent plates, along with DMSO (negative control) and cycloheximide (positive control). Dilutions (all final concentrations) of $35 \mu \mathrm{M}, 31 \mu \mathrm{M}, 27 \mu \mathrm{M}, 23 \mu \mathrm{M}$, $19 \mu \mathrm{M}, 15 \mu \mathrm{M}, 11 \mu \mathrm{M}$ and $7 \mu \mathrm{M}$ for $\mathrm{FC}-888$, and $61 \mu \mathrm{M}, 56 \mu \mathrm{M}, 49 \mu \mathrm{M}, 42 \mu \mathrm{M}, 35 \mu \mathrm{M}, 28 \mu \mathrm{M}, 14$ $\mu \mathrm{M}$ and $7 \mu \mathrm{M}$ for FC-592 were prepared, along with a DMSO control. $100 \mu \mathrm{L}$ aliquots of each of these dilutions (plus control) were dispensed into separate tubes containing the cell suspension, and grown for $\sim 15 \mathrm{~h}$ at $30^{\circ} \mathrm{C}$. Cell titre of each of these cultures was then determined via haemocytometer and cell counts recorded.

\subsubsection{Microarray HOP assay}

The microarray HOP assay was performed based on a protocol by Rosemary Heathcott of the Victoria University of Wellington Chemical Genetics Laboratory, adapted from Parsons et al. 2006. 10 $\mathrm{mL} \mathrm{SC} \mathrm{G418} \mathrm{was} \mathrm{inoculated} \mathrm{with} \mathrm{an} 0.5 \mathrm{~mL}$ aliquot of the HOP Yeast Knockout Pool. This was incubated overnight at $30^{\circ} \mathrm{C}$ on a rotating drum. Cell titre was determined via haemocytometer and diluted to $5 \times 10^{5}$ cells $\mathrm{mL}^{-1}$ with fresh SC G418. This concentration insures at least $1 \times 10^{3}$ examples of each strain from within the pool are present in the starting culture. $10 \mathrm{~mL}$ parallel cultures were treated with $\mathrm{IC}_{30}$ concentrations of FC-592 $(28 \mu \mathrm{M})$ and FC-888 $(11 \mu \mathrm{M})$, alongside $1 \%$ DMSO controls. Cultures were incubated at $30^{\circ} \mathrm{C}$ for $\sim 15 \mathrm{~h}$ (10 cell divisions) and cell titre determined via haemocytometer to ensure $\sim 30 \%$ growth inhibition. These cultures were then re-diluted to $5 \times 10^{5}$ cells $\mathrm{mL}^{-1}$ with fresh SC G418 and treated with $\mathrm{IC}_{30}$ concentrations of the target compounds and the DMSO control for $\sim 15 \mathrm{~h}$ (10 cell cycles). 


\section{DNA purification}

$1.5 \mathrm{~mL}$ of each culture was harvested and genomic DNA purified using the MasterPure Yeast DNA Purification Kit, according to the manufacturer's instructions. The genomic DNA was dissolved in 35 $\mu \mathrm{L}$ TE buffer. DNA was then incubated with $1 \mu \mathrm{L} 5 \mu \mathrm{g} / \mu \mathrm{L}$ RNase $\mathrm{A}$ at $37^{\circ} \mathrm{C}$ for 30 min to remove RNA contamination.

RNase was removed by extracting DNA with Phenol:Chloroform and the combined duplicates made up to $500 \mu \mathrm{L}$ with $\mathrm{ddH}_{2} \mathrm{O} .500 \mu \mathrm{L}$ of phenol/chloroform/isoamylalcohol (25:24:1) was added, the tube vortexed and spun for $10 \mathrm{~min}$ at $16000 \mathrm{~g}$. The aqueous layer (containing the DNA) was removed and 1 volume of chloroform added to it. This mixture was then further vortexed and spun at $16000 \mathrm{~g}$ for $10 \mathrm{~m}$.

The aqueous layer was removed and the DNA precipitated by adding 2.5 volumes $(1 \mathrm{~mL}) 96 \%$ ethanol and $1 / 10$ volume $(40 \mu \mathrm{L}) 3 \mathrm{M}$ sodium acetate $(\mathrm{pH} 5.3)$, and gently mixed by inversion.

This mixture was then cooled at $-20^{\circ} \mathrm{C}$ for $25 \mathrm{~min}$ and spun at $16000 \mathrm{~g}$ for $10 \mathrm{~min}$, before the ethanol layer was poured off and the DNA pellet washed with $0.5 \mathrm{~mL} 70 \%$ ethanol. This was then spun at $16000 \mathrm{~g}$ for $5 \mathrm{~min}$ and the ethanol poured off. The purified DNA pellet was redissolved in $35 \mu \mathrm{LTE}$ buffer.

\section{Quantification of DNA with Hoechst 33258 dye}

$200 \mu \mathrm{L}$ of $2 \mu \mathrm{g} / \mathrm{mL}$ H33258 was pipetted into wells on a 96-well microtitre plate. Calf thymus DNA (Invitrogen) standards were made up at $20 \mathrm{ng} \mathrm{mL}^{-1}, 50 \mathrm{ng} \mathrm{mL}^{-1}, 100 \mathrm{ng} \mathrm{mL}^{-1}, 200 \mathrm{ng} \mathrm{mL}^{-1}, 500 \mathrm{ng} \mathrm{mL}^{-1}$, $1 \mu \mathrm{g} \mathrm{mL} \mathrm{L}^{-1}$ and $2 \mu \mathrm{gL}^{-1}$ concentrations in microtitre wells, and $5 \mu \mathrm{L}$ of each DNA sample was added to separate wells. Fluorescence was measured at $460 \mathrm{~nm}$ on a SpectraMax Plus384 Absorbance Microplate Reader (Molecular Devices) and DNA volumes calculated. DNA aliquots of each sample

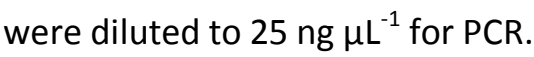




\section{PCR of UP and DN tags with Cy3 and Cy5 dye labelled primers}

Four PCR reactions were carried out to amplify the DNA products. Up (UP) and Down (DN) tags were amplified in separate reactions for both the control and experimental DNA samples. For each treatment (FC-592 \& FC-888) eight PCR reactions were carried out at a final volume of $60 \mu \mathrm{L}$ with final concentrations of $1.5 \mathrm{mM} \mathrm{MgCl} 2,0.2 \mathrm{mM}$ dNTPs, $1 \mu \mathrm{M}$ primers (D1(control), D1(treated),

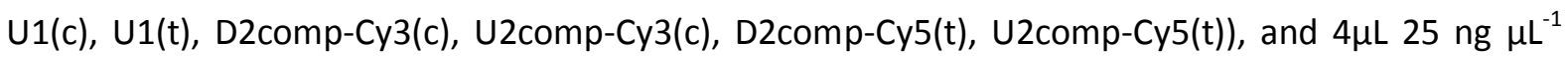
DNA. Eight corresponding negative controls with $\mathrm{ddH}_{2} \mathrm{O}$ instead of DNA were also run to ensure absence of confounding contamination. Following the PCR reactions $5 \mu \mathrm{L}$ of each PCR product was run on a high resolution 4\% MetaPhor agarose gel (Lonza) with $10 \mathrm{bp}$ marker to confirm presence of 56 bp PCR product UP and DN tags.

\section{Precipitation of Cy labelled PCR products}

PCR products were then treated with blocking primers. These complimentary oligonucleotide sequences bind with the priming regions of the PCR products preventing them from binding with each other and allowing them to hybridize to the microarray. $55 \mu \mathrm{L}$ aliquots of the four PCR products were combined with $20 \mu \mathrm{L}$ of blocking mix (12.5 $\mu \mathrm{L} 100 \mu \mathrm{M}$ U1 primer; $12.5 \mu \mathrm{L} 100 \mu \mathrm{M}$ D1 primer; $12.5 \mu \mathrm{L} 100 \mu \mathrm{M}$ U2 block; $12.5 \mu \mathrm{L} 100 \mu \mathrm{M}$ D2 block; $50 \mu \mathrm{L}$ dH20), and vortexed before $24 \mu \mathrm{L}$ of $3 \mathrm{M}$ sodium acetate $\mathrm{pH} 5.2,600 \mu \mathrm{L}$ of absolute ethanol, and $1 \mu \mathrm{L}$ of $5 \mathrm{ng} \mathrm{mL}^{-1}$ linear acrylamide (acts as carrier for precipitation), whereupon the mixture was again vortexed and cooled at $-20^{\circ} \mathrm{C}$ for $1.5 \mathrm{~h}$ to precipitate the Cy labelled PCR products. The mixture was then centrifuged at $4^{\circ} \mathrm{C}$ and $16000 \mathrm{~g}$ for 30 min, the supernatant discarded and the pellet washed with $1 \mathrm{~mL}$ of $70 \%$ ethanol, before again being centrifuged at $4^{\circ} \mathrm{C}$ and $16000 \mathrm{~g}$ for $5 \mathrm{~min}$. At this point the ethanol was removed and the products dried briefly in the absence of light. Finally, the Cy labelled PCR products were redissolved in $50 \mu \mathrm{L}$ $\mathrm{dH}_{2} \mathrm{O}$. 


\section{Microarray hybridisation}

$50 \mu \mathrm{L}$ aliquots of labelled PCR product and blocking mix were added to $50 \mu \mathrm{L} 2 \mathrm{X}$ Hybridisation Buffer (2 $\mathrm{M} \mathrm{NaCl}, 20 \mathrm{mM}$ Tris- $\mathrm{HCl} \mathrm{pH}$ 7.5, 1\% Triton X-100, $1 \mathrm{mM} \mathrm{DTT)}$ and gently pipette mixed. This hybridisation mix was denatutred at $95^{\circ} \mathrm{C}$ for 2 min and applied to the active side of the gasket slide (Agilent Technologies Custom Microarray), sitting in the hybridisation chamber base, according to the instructions given in the Agilent Microarray hybridisation chamber user guide. The microarray slide was placed active side down on the gasket slide, the chamber assembled and placed in the hybridisation oven pre-heated to $42^{\circ} \mathrm{C}$ and set to rotate speed 4 . The completed microarray slide was scanned on a GenePix 4000B microarray scanner at the Otago Genomics Facility, University of Otago, Dunedin, NZ.

\section{Analysing Yeast Tag Microarray Image Data}

Data was extracted and fitted using Genepix 6.0 software, exported in spreadsheet format and normalised using SNOMAD (Standardization and Normalization of Microarray Data, from http://pevsnerlab.kennedykrieger.org/snomaninput.html) software so that the mean ratio across the range of signal intensities is 1 ( 0 on a log scale). The data was then log transformed in base 2 to approximate a normal distribution and mean $\log ($ intensities) and $\log ($ ratios) were graphed to give an M-A plot. Low intensity spots in cy3 $(<500)$ were discarded to reduce data 'noise'. Z-scores were calculated by dividing $\mathrm{x}$-mean scores by standard deviation and ranked to reveal gene hits, which were recorded as being those data points 3 standard deviations or more above the mean.

\subsubsection{Confirmation dose responses}

Selected hits from the FC-592 microarray were assayed to confirm sensitivity. To conserve supplies FC-592, which were extremely limited, only hits related to the pathways being investigated were assayed in this fashion. Single colonies of sla $1 \Delta, \operatorname{mon} 2 \Delta, \operatorname{vps} 74 \Delta, \operatorname{spf} 1 \Delta, \operatorname{gup} 1 \Delta, \operatorname{vps} 35 \Delta, \operatorname{csg} 1 \Delta$, gda1 $\Delta$, ost6 $\Delta$, alg8 $\Delta$, hoc1 $\Delta$ and alg8 $\Delta$ along with a $Y 7092$ control were separately inoculated into 3

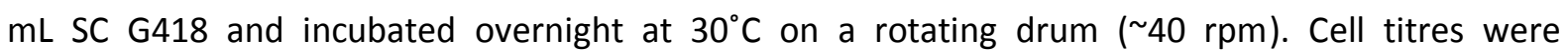


determined via haemocytometer and diluted to $5 \times 10^{5}$ cells $\mathrm{mL}^{-1}$ with fresh SC and vortexed to create a homogeneous suspension. $100 \mu \mathrm{L}$ of media containing cells was dispensed (Eppendorf Xplorer Plus 8 Channel Electronic Pipette) into each well of a clear 96 well flat-bottom polystyrene cell culture plate (Interlab). $1 \mu \mathrm{L}$ aliquots of $28 \mu \mathrm{M}$ (final concentration) FC-596, were dispensed into each well and pipette mixed. Plates were vortexed at $1000 \mathrm{rpm}$ for $30 \mathrm{~s}$ to mix completely and incubated at $30^{\circ} \mathrm{C}$ for $12 \mathrm{~h}$. Following incubation, cultures were resuspended by vortexing at 1000 rpm for $30 \mathrm{~s}$ and the absorbance measured at $590 \mathrm{~nm}$ in a spectrophotometer (EnVision 2102 Multilabel Plate Reader, Perkin Elmer, Waltham, Ma, USA). Residual growth (\%) was determined by comparing the turbidity in each condition against the mean absorbance for DMSO control (Abs590 (experimental)/ Abs590 DMSO (control)) $\times 100$ for each unique condition.

\subsubsection{Gene Ontology (GO) Term analysis}

The Biological Networks Gene Ontology tool (BiNGO) is an open-source Java tool (Maere et al. 2005) to determine which Gene Ontology (GO) terms are significantly overrepresented in a set of genes. This allows statistical analysis of enrichment in a data set for genes within a certain GO term. GO terms exist within three hierarchically structured vocabularies that describe genes and gene products in terms of their associated biological processes, molecular functions and cellular components. 'Hit' genes for FC-592 and FC-888 were assessed according to the publisher's instructions using BiNGO Java plug-in for Cytoscape (http://www.psb.ugent.be/cbd/papers/BiNGO/).

\subsubsection{Unfolded Protein Response expression assay}

A single colony of YCG266 was inoculated into $3 \mathrm{~mL} \mathrm{SC}$ and grown overnight at $30^{\circ} \mathrm{C}$ on a rotating drum. Cell titre was determined via haemocytometer, diluted to $5 \times 10^{6}$ cells $\mathrm{mL}^{-1}$ with fresh SC and vortexed to ensure homogenous suspension. $50 \mu \mathrm{L}$ aliquots of this cell suspension were dispensed into wells in a PerkinElmer 384-well confocal microscopy plate. $8 \mathrm{mM}, 4 \mathrm{mM}, 2 \mathrm{mM}, 1 \mathrm{mM}, 0.500$ $\mathrm{mM}, 0.250 \mathrm{mM}$, and $0.125 \mathrm{mM}$ dilutions (all final concentrations) of DTT along with an untreated control and $112 \mu \mathrm{M}, 56 \mu \mathrm{M}, 28 \mu \mathrm{M}, 14 \mu \mathrm{M}, 7 \mu \mathrm{M}, 3.5 \mu \mathrm{M}$ and $1.75 \mu \mathrm{M}$ dilutions (all final 
concentrations) of FC-592 along with $1 \%$ DMSO and $\mathrm{ddH}_{2} \mathrm{O}$ controls were aliquoted into separate wells. These treated cultures were incubated at $30^{\circ} \mathrm{C}$ for $4 \mathrm{~h}$, before being read with an Opera High Content Screening System confocal microscope (PerkinElmer). Excitation and emission wavelengths were $488 \mathrm{~nm} \& 520 \mathrm{~nm}$ (GFP), and $561 \mathrm{~nm} \& 600 \mathrm{~nm}$ (RFP) respectively. Data from this assay was analysed using Acapella software (PerkinElmer), with $\operatorname{Ln}(\mathrm{GFP} / \mathrm{mCherry)}$ being calculated to determine activation of the UPR.

\subsection{Results}

\subsubsection{Determination of IC 30 concentrations}

Dose response experiments were carried out for FC-592 and FC-888 over a narrow concentration range to determine $\mathrm{IC}_{30}$ concentrations. Cell titres were determined in triplicate via haemocytometer to ensure precision. The following concentrations were selected to proceed on to the microarray phase of the experiment:

\begin{tabular}{|l|r|r|r|}
\hline Compound & Conc. $(\mu \mathrm{M})$ & $\begin{array}{l}\text { Residual growth (\% of } \\
\text { DMSO treated control) }\end{array}$ & Standard Deviation \\
\hline FC-592 & 28 & 74.81 & 6.75 \\
\hline FC-888 & 11 & 83.00 & 4.26 \\
\hline
\end{tabular}

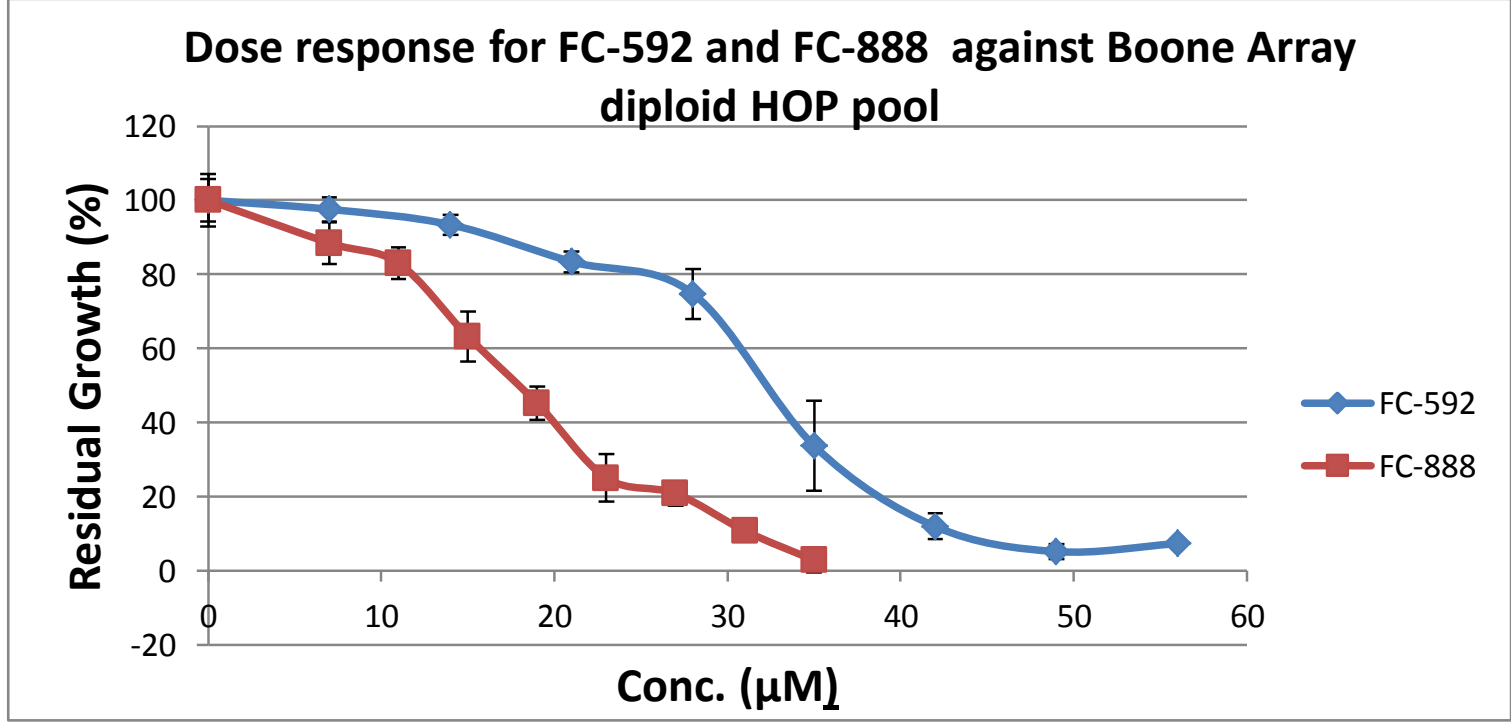

Figure 3.4 Dose responses were conducted over a single order of magnitude to precisely determine $\sim \mathrm{IC}_{30}$ concentrations. $28 \mu \mathrm{M}$ FC-592 was found to inhibit $25 \%$ growth and $11 \mu \mathrm{M}$ FC-888 was found to inhibit $17 \%$ growth. Note that these values do not correspond exactly with results obtained in earlier dose responses, as diploid cells are being used in this experiment. 


\subsubsection{Microarray Chemigenomic Profiling}

Chemigenomic profiles of FC-592 and FC-888 were generated using a yeast tag microarray based on the pooled homozygous diploid deletion mutant array (HOM DMA). These deletion mutant pools were competitively grown in the presence of $28 \mu \mathrm{M} F C-592$ and $11 \mu \mathrm{M} \mathrm{FC}-888$, respectively. Genomic DNA for the pooled strains was extracted and amplified via PCR using barcode tag specific primers to generate a pool of oligomers representing strains grown in competitive conditions within the pool. Successful PCR was confirmed via agarose gel electrophoresis, before hybridization to the microarray chip.

4713 DN tags and 3468 UP tags were successfully recorded for FC-592 after processing and normalisation of data. For FC-888, 3625 Dn tags and 3383 Up tags were successfully recorded. Genes represented by these tags were ranked according to their underrepresentation within the nonessential yeast genome set, with those recording Z-scores (standard deviations from the mean) recorded as 'hits'. Genes implicated in multidrug resistance (Hillenmeyer et al. 2008) were removed from this analysis as they are unlikely to contribute to a specific sensitivity mechanism with regards to an individual compound.

FC-592 Chemigenomic profile 'Hits'

\begin{tabular}{|c|c|c|c|c|c|}
\hline ORF & Gene & Tag type & Z-score & Component & Biological function \\
\hline YOR002W & ALG6 & Up & -6.07403 & 1 & $\begin{array}{l}\text { Alpha } 1,3 \text { glucosyltransferase, involved in transfer of } \\
\text { oligosaccharides from dolichyl pyrophosphate to asparagine } \\
\text { residues of proteins during N-linked protein glycosylation; } \\
\text { mutations in human ortholog are associated with disease }\end{array}$ \\
\hline YOR002W & ALG6 & Dn & -3.73824 & " & \\
\hline YOR067C & ALG8 & Up & -3.75501 & 1 & $\begin{array}{l}\text { Glucosyl transferase, involved in } \mathrm{N} \text {-linked glycosylation; adds } \\
\text { glucose to the dolichol-linked oligosaccharide precursor prior to } \\
\text { transfer to protein during lipid-linked oligosaccharide biosynthesis; } \\
\text { similar to Alg6p }\end{array}$ \\
\hline YOR067C & ALG8 & Dn & -3.12691 & $"$ & \\
\hline YGL148W & ARO2 & Dn & -4.20699 & 2 & $\begin{array}{l}\text { Bifunctional chorismate synthase and flavin reductase, catalyzes } \\
\text { the conversion of } 5 \text {-enolpyruvylshikimate } 3 \text {-phosphate (EPSP) to } \\
\text { form chorismate, which is a precursor to aromatic amino acids }\end{array}$ \\
\hline YPR060C & ARO7 & Dn & -4.85873 & 2 & $\begin{array}{l}\text { Chorismate mutase, catalyzes the conversion of chorismate to } \\
\text { prephenate to initiate the tyrosine/phenylalanine-specific branch } \\
\text { of aromatic amino acid biosynthesis }\end{array}$ \\
\hline
\end{tabular}




\begin{tabular}{|c|c|c|c|c|c|}
\hline YBR036C & CSG2 & $\mathrm{Dn}$ & -8.78656 & 1 & $\begin{array}{l}\text { Endoplasmic reticulum membrane protein, required for } \\
\text { mannosylation of inositolphosphorylceramide and for growth at } \\
\text { high calcium concentrations }\end{array}$ \\
\hline YMR202W & ERG2 & Dn & -3.94833 & 3 & $\begin{array}{l}\text { C-8 sterol isomerase, catalyzes the isomerization of the delta- } 8 \\
\text { double bond to the delta- } 7 \text { position at an intermediate step in } \\
\text { ergosterol biosynthesis }\end{array}$ \\
\hline YCR034W & FEN1 & $\mathrm{Dn}$ & -4.87421 & 3 & $\begin{array}{l}\text { Fatty acid elongase, involved in sphingolipid biosynthesis; acts on } \\
\text { fatty acids of up to } 24 \text { carbons in length; mutations have } \\
\text { regulatory effects on } 1,3 \text {-beta-glucan synthase, vacuolar ATPase, } \\
\text { and the secretory pathway }\end{array}$ \\
\hline YCR034W & FEN1 & Up & -4.16013 & $"$ & \\
\hline YOL051W & GAL11 & Up & -3.03883 & 4 & $\begin{array}{l}\text { Subunit of the RNA polymerase II mediator complex; associates } \\
\text { with core polymerase subunits to form the RNA polymerase II } \\
\text { holoenzyme; affects transcription by acting as target of activators } \\
\text { and repressors }\end{array}$ \\
\hline YEL042W & GDA1 & Up & -6.95308 & 1 & $\begin{array}{l}\text { Guanosine diphosphatase located in the Golgi, involved in the } \\
\text { transport of GDP-mannose into the Golgi lumen by converting GDP } \\
\text { to GMP after mannose is transferred its substrate }\end{array}$ \\
\hline YEL042W & GDA1 & Dn & -5.85025 & " & \\
\hline YGL084C & GUP1 & $\mathrm{Dn}$ & -6.43054 & 1 & $\begin{array}{l}\text { Plasma membrane protein involved in remodeling GPI anchors; } \\
\text { member of the MBOAT family of putative membrane-bound O- } \\
\text { acyltransferases; proposed to be involved in glycerol transport }\end{array}$ \\
\hline YJR075W & HOC1 & $\mathrm{Dn}$ & -4.59351 & 1 & $\begin{array}{l}\text { Alpha-1,6-mannosyltransferase involved in cell wall mannan } \\
\text { biosynthesis; subunit of a Golgi-localized complex that also } \\
\text { contains Anp1p, Mnn9p, Mnn11p, and Mnn10p; identified as a } \\
\text { suppressor of a cell lysis sensitive pkc1-371 allele }\end{array}$ \\
\hline YOL081W & IRA2 & Up & -3.01241 & 2 & $\begin{array}{l}\text { GTPase-activating protein that negatively regulates RAS by } \\
\text { converting it from the GTP- to the GDP-bound inactive form, } \\
\text { required for reducing CAMP levels under nutrient limiting } \\
\text { conditions, has similarity to Ira1p and human neurofibromin }\end{array}$ \\
\hline YPR067W & ISA2 & Dn & -3.56875 & 5 & $\begin{array}{l}\text { Protein required for maturation of mitochondrial and cytosolic } \\
\mathrm{Fe} / \mathrm{S} \text { proteins, localizes to the mitochondrial intermembrane space, } \\
\text { overexpression of ISA2 suppresses grx5 mutations }\end{array}$ \\
\hline YJL124C & LSM1 & Up & -3.8604 & 4 & $\begin{array}{l}\text { Lsm (Like } S m \text { ) protein; forms heteroheptameric complex (with } \\
\operatorname{Lsm} 2 p \text {, Lsm3p, Lsm4p, Lsm5p, Lsm6p, and } L s m 7 p \text { ) involved in } \\
\text { degradation of cytoplasmic mRNAs }\end{array}$ \\
\hline YNL147W & LSM7 & Up & -3.03715 & 4 & $\begin{array}{l}\text { Lsm (Like } S \mathrm{~m} \text { ) protein; part of heteroheptameric complexes } \\
\text { (Lsm2p-7p and either Lsm1p or } 8 \mathrm{p}) \text { : cytoplasmic Lsm1p complex } \\
\text { involved in mRNA decay; nuclear Lsm8p complex part of U6 snRNP } \\
\text { and possibly involved in processing tRNA, snoRNA, and rRNA }\end{array}$ \\
\hline YPL187W & $\mathrm{MF}(\alpha) 1$ & Dn & -4.50944 & 6 & $\begin{array}{l}\text { Mating pheromone alpha-factor, made by alpha cells; interacts } \\
\text { with mating type a cells to induce cell cycle arrest and other } \\
\text { responses leading to mating; also encoded by MF(ALPHA)2, } \\
\text { although MF(ALPHA)1 produces most alpha-factor }\end{array}$ \\
\hline YER028C & MIG3 & Dn & -4.00841 & 4 & $\begin{array}{l}\text { Probable transcriptional repressor involved in response to toxic } \\
\text { agents such as hydroxyurea that inhibit ribonucleotide reductase; } \\
\text { phosphorylation by Snf1p or the Mec1p pathway inactivates } \\
\text { Mig3p, allowing induction of damage response genes }\end{array}$ \\
\hline YNL297C & MON2 & Up & -5.3557 & 1 & $\begin{array}{l}\text { Peripheral membrane protein with a role in endocytosis and } \\
\text { vacuole integrity, interacts with Arl1p and localizes to the } \\
\text { endosome; member of the Sec7p family of proteins }\end{array}$ \\
\hline YNL297C & MON2 & Dn & -4.8626 & " & \\
\hline YGR165W & MRPS35 & Up & -3.05333 & 5 & Mitochondrial ribosomal protein of the small subunit \\
\hline YHL023C & NPR3 & Up & -3.15708 & 2 & $\begin{array}{l}\text { Subunit of SEA (Seh1-associated), Npr } 2 / 3 \text {, and Iml1p complexes; } \\
\text { Npr2/3 complex mediates downregulation of TORC1 activity upon } \\
\text { amino acid limitation; SEA complex is a coatomer-related complex } \\
\text { that associates dynamically with the vacuole; Iml1p complex } \\
\text { (Iml1p-Npr2p-Npr3p) is required for non-nitrogen-starvation } \\
\text { (NNS)-induced autophagy; required for Npr2p phosphorylation and } \\
\text { Iml1p-Npr2p interaction; null mutant shows delayed meiotic DNA } \\
\text { replication and double-strand break repair }\end{array}$ \\
\hline YLR338W & OPI9 & Dn & -3.18778 & 6 & $\begin{array}{l}\text { Dubious open reading frame unlikely to encode a protein, based } \\
\text { on available experimental and comparative sequence data; } \\
\text { partially overlaps the verified ORF VRP } 1 / \text { Y RR } 337 C\end{array}$ \\
\hline YML019W & OST6 & Up & -3.01031 & 1 & $\begin{array}{l}\text { Subunit of the oligosaccharyltransferase complex of the ER lumen, } \\
\text { which catalyzes asparagine-linked glycosylation of newly } \\
\text { synthesized proteins; similar to and partially functionally }\end{array}$ \\
\hline
\end{tabular}




\begin{tabular}{|c|c|c|c|c|c|}
\hline & & & & & redundant with Ost3p \\
\hline YCR077C & PAT1 & Dn & -3.32008 & 4 & $\begin{array}{l}\text { Topoisomerase II-associated deadenylation-dependent mRNA- } \\
\text { decapping factor; also required for faithful chromosome } \\
\text { transmission, maintenance of rDNA locus stability, and protection } \\
\text { of mRNA 3'-UTRs from trimming; functionally linked to Pab1p }\end{array}$ \\
\hline YBL051C & PIN4 & Dn & -3.98754 & 6 & $\begin{array}{l}\text { Protein involved in G2/M phase progression and response to DNA } \\
\text { damage, interacts with Rad53p; contains an RNA recognition motif, } \\
\text { a nuclear localization signal, and several SQ/TQ cluster domains; } \\
\text { hyperphosphorylated in response to DNA damage }\end{array}$ \\
\hline YBL051C & PIN4 & Up & -3.9741 & $"$ & \\
\hline YDR289C & RTT103 & $\mathrm{Dn}$ & -3.28213 & 4 & $\begin{array}{l}\text { Protein that interacts with exonuclease Rat1p and Raip and plays } \\
\text { a role in transcription termination by RNA polymerase II, has an } \\
\text { RPR domain (carboxy-terminal domain interacting domain); also } \\
\text { involved in regulation of Ty1 transposition }\end{array}$ \\
\hline YBL007C & SLA1 & Dn & -3.30793 & 1 & $\begin{array}{l}\text { Cytoskeletal protein binding protein required for assembly of the } \\
\text { cortical actin cytoskeleton; interacts with proteins regulating actin } \\
\text { dynamics and proteins required for endocytosis; found in the } \\
\text { nucleus and cell cortex; has } 3 \text { SH3 domains }\end{array}$ \\
\hline YOR290C & SNF2 & Up & -8.30774 & 4 & $\begin{array}{l}\text { Catalytic subunit of the SWI/SNF chromatin remodelling complex } \\
\text { involved in transcriptional regulation; contains DNA-stimulated } \\
\text { ATPase activity; functions interdependently in transcriptional } \\
\text { activation with Snf5p and Snf6p }\end{array}$ \\
\hline YOR290C & SNF2 & Dn & -5.43934 & $"$ & \\
\hline YBR289W & SNF5 & $\mathrm{Dn}$ & -3.17721 & 4 & $\begin{array}{l}\text { Subunit of the SWI/SNF chromatin remodelling complex involved } \\
\text { in transcriptional regulation; functions interdependently in } \\
\text { transcriptional activation with Snf2p and Snf6p }\end{array}$ \\
\hline YEL031W & SPF1 & Dn & -3.02362 & 1 & $\begin{array}{l}\text { P-type ATPase, ion transporter of the ER membrane involved in ER } \\
\text { function and Ca2+ homeostasis; required for regulating Hmg2p } \\
\text { degradation; confers sensitivity to a killer toxin (SMKT) produced } \\
\text { by Pichia farinosa KK1 }\end{array}$ \\
\hline YHR041C & SRB2 & $\mathrm{Dn}$ & -4.35678 & 4 & $\begin{array}{l}\text { Subunit of the RNA polymerase } \| \text { mediator complex; associates } \\
\text { with core polymerase subunits to form the RNA polymerase } \| \\
\text { holoenzyme; general transcription factor involved in telomere } \\
\text { maintenance }\end{array}$ \\
\hline YBL054W & TOD6 & Dn & -3.85573 & 4 & $\begin{array}{l}\text { PAC motif binding protein involved in rRNA and ribosome } \\
\text { biogenesis; subunit of the RPD3L histone deacetylase complex; } \\
\text { Myb-like HTH transcription factor, similar to Dot6p; } \\
\text { hypophosphorylated by rapamycin treatment in a Sch9p- } \\
\text { dependent manne }\end{array}$ \\
\hline YLR373C & VID22 & $\mathrm{Dn}$ & -3.69627 & 6 & $\begin{array}{l}\text { Glycosylated integral membrane protein localized to the plasma } \\
\text { membrane; plays a role in fructose- } 1,6 \text {-bisphosphatase (FBPase) } \\
\text { degradation; involved in FBPase transport from the cytosol to Vid } \\
\text { (vacuole import and degradation) vesicles }\end{array}$ \\
\hline YJL154C & VPS35 & Up & -3.31256 & 1 & $\begin{array}{l}\text { Endosomal subunit of membrane-associated retromer complex } \\
\text { required for retrograde transport; receptor that recognizes } \\
\text { retrieval signals on cargo proteins, forms subcomplex with Vps26p } \\
\text { and Vps29p that selects cargo proteins for retrieval }\end{array}$ \\
\hline YDR372C & VPS74 & Dn & -3.23362 & 1 & $\begin{array}{l}\text { Protein required for Golgi localization of glycosyltransferases; } \\
\text { binds the cytosolic domains of Golgi glycosyltransferases; binding } \\
\text { to Ptdlns4P required for Golgi targeting and function; tetramer } \\
\text { formation required for function }\end{array}$ \\
\hline YNL246W & VPS75 & Dn & -3.13105 & 6 & $\begin{array}{l}\text { NAP family histone chaperone; binds to histones and Rtt109p, } \\
\text { stimulating histone acetyltransferase activity; possesses } \\
\text { nucleosome assembly activity in vitro; proposed role in vacuolar } \\
\text { protein sorting and in double-strand break repair }\end{array}$ \\
\hline YER123W & YCK3 & Dn & -3.48264 & 6 & $\begin{array}{l}\text { Palmitoylated, vacuolar membrane-localized casein kinase } \\
\text { isoform; negatively regulates vacuole fusion during hypertonic } \\
\text { stress via phosphorylation of Vps41p; shares essential functions } \\
\text { with Hrr25p; regulates vesicle fusion in AP-3 pathway }\end{array}$ \\
\hline YDL118W & - & Up & -3.21129 & 6 & $\begin{array}{l}\text { Non-essential protein of unconfirmed function; mutants are } \\
\text { defective in telomere maintenance, and are synthetically sick or } \\
\text { lethal with alpha-synuclein }\end{array}$ \\
\hline YMR010W & - & Up & -3.82676 & 6 & $\begin{array}{l}\text { Putative protein of unknown function; green fluorescent protein } \\
\text { (GFP)-fusion protein localizes to the cytoplasm; YMRO10W is not } \\
\text { an essential gene; YMRO1OW mRNA is transcribed with ADI1 }\end{array}$ \\
\hline YNL170W & - & Dn & -4.52595 & 6 & $\begin{array}{l}\text { Dubious open reading frame unlikely to encode a functional } \\
\text { protein, based on available experimental and comparative }\end{array}$ \\
\hline
\end{tabular}


YOL087C

YHR155W

YSP1

Dn

Dn

$-4.44032$
6

5
Putative protein of unknown function; green fluorescent protein (GFP)-fusion protein localizes to the cytoplasm; deletion mutant is sensitive to various chemicals including phenanthroline, sanguinarine, and nordihydroguaiaretic acid

Mitochondrial protein with a potential role in promoting mitochondrial fragmentation during programmed cell death in response to high levels of alpha-factor mating pheromone or the drug amiodarone

Table 3.1 List of hypersensitive mutants derived from HOP screen of FC-592 treated cells. 48 tags were identified that exceeded the threshold of three standard deviations from the mean. These tags represent 41 unique genes with seven represented by both Up and Dn tags. Genes were grouped according to cellular component/process: Group 1: ER/Golgi; 2: Nutrient/amino acid biosynthesis; 3: Cell wall; 4: Transcription ; 5: Mitochondria; 6: Miscellaneous components. Genes highlighted in red are linked with glycoprotein biosynthesis and/or the secretory pathway.

FC-888 Chemigenetic profile 'Hits'

\begin{tabular}{|c|c|c|c|c|c|}
\hline ORF & Gene & $\begin{array}{l}\text { Tag } \\
\text { type }\end{array}$ & Z-score & Component & Biological function \\
\hline YNR074C & AlF1 & Dn & -3.19792 & 2 & $\begin{array}{l}\text { Mitochondrial cell death effector that translocates to the nucleus in response to } \\
\text { apoptotic stimuli, homolog of mammalian Apoptosis-Inducing Factor, putative } \\
\text { reductase }\end{array}$ \\
\hline YDR530C & APA2 & Up & -3.17042 & 7 & $\begin{array}{l}\text { Diadenosine } 5 \text { ',5"-P1,P4-tetraphosphate phosphorylase II (AP4A phosphorylase), } \\
\text { involved in catabolism of bis(5'-nucleosidyl) tetraphosphates; has similarity to } \\
\text { Apa1p }\end{array}$ \\
\hline YDR530C & APA2 & $\mathrm{Dn}$ & -3.1421 & " & " \\
\hline YJL095W & BCK1 & Dn & -3.31904 & 3 & $\begin{array}{l}\text { Mitogen-activated protein (MAP) kinase kinase kinase acting in the protein kinase } \\
\mathrm{C} \text { signaling pathway, which controls cell integrity; upon activation by Pkc1p } \\
\text { phosphorylates downstream kinases Mkk1p and Mkk2p }\end{array}$ \\
\hline YJL095W & BCK1 & Up & -3.15677 & " & " \\
\hline YBL085W & BOI1 & Up & -3.3999 & 5 & $\begin{array}{l}\text { Protein implicated in polar growth, functionally redundant with Boi2p; interacts } \\
\text { with bud-emergence protein Bem1p; contains an SH3 (src homology 3) domain and } \\
\text { a PH (pleckstrin homology) domain }\end{array}$ \\
\hline YNR051C & BRE5 & Dn & -3.85368 & 6 & $\begin{array}{l}\text { Ubiquitin protease cofactor, forms deubiquitination complex with Ubp } 3 p \text { that } \\
\text { coregulates anterograde and retrograde transport between the endoplasmic } \\
\text { reticulum and Golgi compartments; null is sensitive to brefeldin A }\end{array}$ \\
\hline YOR061W & CKA2 & Dn & -3.11888 & 3 & $\begin{array}{l}\text { Alpha' catalytic subunit of casein kinase } 2 \text { (CK2), a Ser/Thr protein kinase with roles } \\
\text { in cell growth and proliferation; CK2, comprised of CKA1, CKA2, CKB1 and CKB2, } \\
\text { has many substrates including transcription factors and all RNA polymerase }\end{array}$ \\
\hline YPL256C & CLN2 & Up & -3.51291 & 5 & $\begin{array}{l}\text { G1 cyclin involved in regulation of the cell cycle; activates Cdc28p kinase to } \\
\text { promote the G1 to S phase transition; late G1 specific expression depends on } \\
\text { transcription factor complexes, MBF (Swi6p-Mbp1p) and SBF (Swi6p-Swi4p) }\end{array}$ \\
\hline YIR023W & DAL81 & $\mathrm{Dn}$ & -3.40635 & 7 & $\begin{array}{l}\text { Positive regulator of genes in multiple nitrogen degradation pathways; contains } \\
\text { DNA binding domain but does not appear to bind the dodecanucleotide sequence } \\
\text { present in the promoter region of many genes involved in allantoin catabolism }\end{array}$ \\
\hline YIR023W & DAL81 & Up & -3.3413 & " & " \\
\hline YDR385W & EFT2 & Dn & -3.27818 & 8 & $\begin{array}{l}\text { Elongation factor } 2 \text { (EF-2), also encoded by EFT1; catalyzes ribosomal translocation } \\
\text { during protein synthesis; contains diphthamide, the unique posttranslationally } \\
\text { modified histidine residue specifically ADP-ribosylated by diphtheria toxin }\end{array}$ \\
\hline YKL160W & ELF1 & Dn & -3.75475 & 9 & $\begin{array}{l}\text { Transcription elongation factor that contains a conserved zinc finger domain; } \\
\text { implicated in the maintenance of proper chromatin structure in actively } \\
\text { transcribed regions; deletion inhibits Brome mosaic virus (BMV) gene expression }\end{array}$ \\
\hline YKL160W & ELF1 & Up & -3.70621 & $"$ & " \\
\hline YMR222C & $\mathrm{FSH} 2$ & Dn & -3.92239 & 10 & Putative serine hydrolase that localizes to the cytoplasm; sequence is similar to $\mathrm{S}$. \\
\hline
\end{tabular}




\begin{tabular}{|llll|}
\hline YMR222C & FSH2 & Up & -3.6408 \\
\hline YGL020C & GET1 & Dn & -3.0561
\end{tabular}

" "

\begin{tabular}{|c|c|c|c|c|c|}
\hline YGL020C & GET1 & Dn & -3.0561 & 11 & $\begin{array}{l}\text { membrane; required for the retrieval of HDEL proteins from the Golgi to the ER in } \\
\text { an ERD2 dependent fashion and for normal mitochondrial morphology and } \\
\text { inheritance }\end{array}$ \\
\hline YDR508C & GNP1 & Up & -6.3278 & 6 & $\begin{array}{l}\text { High-affinity glutamine permease, also transports Leu, Ser, Thr, Cys, Met and Asn; } \\
\text { expression is fully dependent on Grr1p and modulated by the Ssy1p-Ptr3p-Ssy5p } \\
\text { (SPS) sensor of extracellular amino acids }\end{array}$ \\
\hline YPL223C & GRE1 & Up & -4.02055 & 12 & $\begin{array}{l}\text { Hydrophilin of unknown function; stress induced (osmotic, ionic, oxidative, heat } \\
\text { shock and heavy metals); regulated by the HOG pathway }\end{array}$ \\
\hline YPL223C & GRE1 & Dn & -3.50265 & $"$ & " \\
\hline YOL089C & HAL9 & Up & -3.30429 & 9 & $\begin{array}{l}\text { Putative transcription factor containing a zinc finger; overexpression increases salt } \\
\text { tolerance through increased expression of the ENA1 ( } \mathrm{Na}+\mathrm{Li}+\text { extrusion pump) } \\
\text { gene while gene disruption decreases both salt tolerance and ENA1 expression }\end{array}$ \\
\hline YNL021W & HDA1 & Dn & -4.20945 & 9 & $\begin{array}{l}\text { Putative catalytic subunit of a class II histone deacetylase complex that also } \\
\text { contains } \mathrm{Hda} 2 \mathrm{p} \text { and } \mathrm{Hda} 3 \mathrm{p} ; \mathrm{Hda} 1 \mathrm{p} \text { interacts with the } \mathrm{Hda} 2 \mathrm{p}-\mathrm{Hda} 3 \mathrm{p} \text { subcomplex to } \\
\text { form an active tetramer; deletion increases histone } \mathrm{H} 2 \mathrm{~B}, \mathrm{H} 3 \text { and } \mathrm{H} 4 \text { acetylation }\end{array}$ \\
\hline YDR295C & HDA2 & Dn & -3.19602 & 9 & $\begin{array}{l}\text { Subunit of a possibly tetrameric trichostatin A-sensitive class II histone deacetylase } \\
\text { complex containing an Hda1p homodimer and an Hda2p-Hda3p heterodimer; } \\
\text { involved in telomere maintenance }\end{array}$ \\
\hline YOL013C & HRD1 & Up & -4.15564 & 11 & $\begin{array}{l}\text { Ubiquitin-protein ligase required for endoplasmic reticulum-associated } \\
\text { degradation (ERAD) of misfolded proteins; genetically linked to the unfolded } \\
\text { protein response (UPR); regulated through association with } \mathrm{Hrd} 3 \mathrm{p} \text {; contains an } \mathrm{H} 2 \\
\text { ring finger }\end{array}$ \\
\hline YPL244C & HUT1 & Up & -3.00916 & 11 & $\begin{array}{l}\text { Protein with a role in UDP-galactose transport to the Golgi lumen, has similarity to } \\
\text { human UDP-galactose transporter UGTrel1, exhibits a genetic interaction with S. } \\
\text { cerevisiae ERO1 }\end{array}$ \\
\hline YPR006C & $\mathrm{ICL} 2$ & Up & -5.66125 & 1 & $\begin{array}{l}\text { 2-methylisocitrate lyase of the mitochondrial matrix, functions in the methylcitrate } \\
\text { cycle to catalyze the conversion of } 2 \text {-methylisocitrate to succinate and pyruvate; } \\
\text { ICL2 transcription is repressed by glucose and induced by ethanol }\end{array}$ \\
\hline YPR006C & ICL2 & Dn & -4.38872 & $"$ & " \\
\hline YIR005W & IST3 & Up & -3.02225 & 12 & $\begin{array}{l}\text { Component of the U2 snRNP, required for the first catalytic step of splicing and for } \\
\text { spliceosomal assembly; interacts with Rds3p and is required for Mer1p-activated } \\
\text { splicing }\end{array}$ \\
\hline YKL110C & KTI12 & Up & -3.0078 & 5 & $\begin{array}{l}\text { Protein that plays a role, with Elongator complex, in modification of wobble } \\
\text { nucleosides in tRNA; involved in sensitivity to G1 arrest induced by zymocin; } \\
\text { interacts with chromatin throughout the genome; also interacts with Cdc19p }\end{array}$ \\
\hline YKL183W & LOT5 & Dn & -3.52908 & 12 & $\begin{array}{l}\text { Protein of unknown function; gene expression increases in cultures shifted to a } \\
\text { lower temperature }\end{array}$ \\
\hline YKL183W & LOT5 & Up & -3.44937 & $"$ & " \\
\hline YKL176C & LST4 & Dn & -4.02574 & 6 & $\begin{array}{l}\text { Protein possibly involved in a post-Golgi secretory pathway; required for the } \\
\text { transport of nitrogen-regulated amino acid permease Gap1p from the Golgi to the } \\
\text { cell surface }\end{array}$ \\
\hline YKL176C & LST4 & Up & -3.75203 & $"$ & " \\
\hline YMR036C & MIH1 & Up & -3.65194 & 5 & $\begin{array}{l}\text { Protein tyrosine phosphatase involved in cell cycle control; regulates the } \\
\text { phosphorylation state of Cdc } 28 \mathrm{p} \text {; homolog of S. pombe cdc } 25\end{array}$ \\
\hline YKR095W & MLP1 & Dn & -3.06369 & 4 & $\begin{array}{l}\text { Myosin-like protein associated with the nuclear envelope, connects the nuclear } \\
\text { pore complex with the nuclear interior; involved with Tel1p in telomere length } \\
\text { control; involved with Pml1p and Pml39p in nuclear retention of unspliced mRNAs }\end{array}$ \\
\hline YBL049W & MOH1 & Up & -3.38468 & 12 & $\begin{array}{l}\text { Protein of unknown function, has homology to kinase Snf7p; not required for } \\
\text { growth on nonfermentable carbon sources; essential for survival in stationary } \\
\text { phase }\end{array}$ \\
\hline YMR070W & MOT3 & Up & -4.81804 & 12 & $\begin{array}{l}\text { Nuclear transcription factor with two Cys2-His2 zinc fingers; involved in repression } \\
\text { of a subset of hypoxic genes by Rox1p, repression of several DAN/TIR genes during } \\
\text { aerobic growth, and repression of ergosterol biosynthetic genes }\end{array}$ \\
\hline YMR070W & MOT3 & Dn & -3.97435 & $"$ & " \\
\hline YML103C & NUP188 & Up & -3.94728 & 4 & $\begin{array}{l}\text { Subunit of the nuclear pore complex (NPC), involved in the structural organization } \\
\text { of the complex and of the nuclear envelope, also involved in nuclear envelope } \\
\text { permeability, interacts with Pom } 152 p \text { and Nic } 96 p\end{array}$ \\
\hline YMR153W & NUP53 & Up & -3.2464 & 4 & $\begin{array}{l}\text { Subunit of the nuclear pore complex (NPC), interacts with karyopherin Kap121p or } \\
\text { with Nup170p via overlapping regions of Nup53p, involved in activation of the } \\
\text { spindle checkpoint mediated by the Mad1p-Mad2p complex }\end{array}$ \\
\hline YGR178C & PBP1 & Dn & -3.96506 & 12 & Component of glucose deprivation induced stress granules, involved in P-body- \\
\hline
\end{tabular}




\begin{tabular}{|c|c|c|c|c|c|}
\hline & & & & & $\begin{array}{l}\text { dependent granule assembly; similar to human ataxin-2; interacts with Pab1p to } \\
\text { regulate mRNA polyadenylation; interacts with Mkt1p to regulate HO translation }\end{array}$ \\
\hline YGR178C & PBP1 & Up & -3.89031 & " & " \\
\hline YDR079W & PET100 & $\mathrm{Dn}$ & -3.41509 & 1 & $\begin{array}{l}\text { Chaperone that specifically facilitates the assembly of cytochrome c oxidase, } \\
\text { integral to the mitochondrial inner membrane; interacts with a subcomplex of } \\
\text { subunits VII, VIIa, and VIII (Cox7p, Cox9p, and Cox8p) but not with the holoenzyme }\end{array}$ \\
\hline YGL153W & PEX14 & $\mathrm{Dn}$ & -3.49637 & 6 & $\begin{array}{l}\text { Peroxisomal membrane peroxin that is a central component of the peroxisomal } \\
\text { protein import machinery; interacts with both PTS1 (Pex5p) and PTS2 (Pex7p), } \\
\text { peroxisomal matrix protein signal recognition factors and membrane receptor } \\
\text { Pex13p }\end{array}$ \\
\hline YGL153W & PEX14 & Up & -3.42615 & $"$ & " \\
\hline YGR077C & PEX8 & $\mathrm{Dn}$ & -3.75389 & 6 & $\begin{array}{l}\text { Intraperoxisomal organizer of the peroxisomal import machinery, tightly associated } \\
\text { with the lumenal face of the peroxisomal membrane, essential for peroxisome } \\
\text { biogenesis, binds PTS1-signal receptor Pex5p }\end{array}$ \\
\hline YIL107C & PFK26 & Up & -3.50845 & 3 & $\begin{array}{l}\text { 6-phosphofructo-2-kinase, inhibited by phosphoenolpyruvate and sn-glycerol 3- } \\
\text { phosphate; has negligible fructose-2,6-bisphosphatase activity; transcriptional } \\
\text { regulation involves protein kinase A }\end{array}$ \\
\hline YBR092C & PHO3 & Dn & -4.44967 & 12 & $\begin{array}{l}\text { Constitutively expressed acid phosphatase similar to Pho5p; brought to the cell } \\
\text { surface by transport vesicles; hydrolyzes thiamin phosphates in the periplasmic } \\
\text { space, increasing cellular thiamin uptake; expression is repressed by thiamin }\end{array}$ \\
\hline YBR092C & $\mathrm{PHO3}$ & Up & -3.25713 & $"$ & " \\
\hline YOR104W & PIN2 & Dn & -4.25829 & 12 & $\begin{array}{l}\text { Protein that induces appearance of }[\mathrm{PIN}+] \text { prion when overproduced; predicted to } \\
\text { be palmitoylated }\end{array}$ \\
\hline YBL051C & PIN4 & Up & -4.63939 & 5 & $\begin{array}{l}\text { Protein involved in } \mathrm{G} 2 / \mathrm{M} \text { phase progression and response to DNA damage, } \\
\text { interacts with Rad53p; contains an RNA recognition motif, a nuclear localization } \\
\text { signal, and several SQ/TQ cluster domains; hyperphosphorylated in response to } \\
\text { DNA damage }\end{array}$ \\
\hline YML017W & PSP2 & Up & -3.16927 & 12 & $\begin{array}{l}\text { Asn rich cytoplasmic protein that contains RGG motifs; high-copy suppressor of } \\
\text { group II intron-splicing defects of a mutation in MRS2 and of a conditional } \\
\text { mutation in POL1 (DNA polymerase alpha); possible role in mitochondrial mRNA } \\
\text { splicing }\end{array}$ \\
\hline YBL046W & PSY4 & Up & -3.79219 & 3 & $\begin{array}{l}\text { Regulatory subunit of protein phosphatase PP4; presence of Psy4p in the PP4 } \\
\text { complex (along with catalytic subunit Pph3p and Psy2p) is required for } \\
\text { dephosphorylation of the histone variant } \mathrm{H} 2 \mathrm{AX} \text {, but not for dephosphorylation of } \\
\text { Rad53p, during recovery from the DNA damage checkpoint; localization is cell-cycle } \\
\text { dependent and regulated by Cdc28p phosphorylation; required for cisplatin } \\
\text { resistance; homolog of mammalian R2 }\end{array}$ \\
\hline YJL204C & RCY1 & $\mathrm{Dn}$ & -4.00186 & 12 & $\begin{array}{l}\text { F-box protein involved in recycling plasma membrane proteins internalized by } \\
\text { endocytosis; localized to sites of polarized growth }\end{array}$ \\
\hline YJL204C & RCY1 & Up & -3.1302 & $"$ & " \\
\hline YBR030W & RKM3 & Up & -3.53898 & 8 & $\begin{array}{l}\text { Ribosomal lysine methyltransferase specific for monomethylation of Rpl42ap and } \\
\text { Rpl42bp (Iysine 40); nuclear SET domain containing protein }\end{array}$ \\
\hline YMR242C & RPL20A & Up & -3.28416 & 8 & $\begin{array}{l}\text { Protein component of the large }(60 S) \text { ribosomal subunit, nearly identical to } \\
\text { Rpl20Bp and has similarity to rat L18a ribosomal protein }\end{array}$ \\
\hline YGL147C & RPL9A & Up & -3.01469 & 8 & $\begin{array}{l}\text { Protein component of the large (60S) ribosomal subunit, nearly identical to Rpl9Bp } \\
\text { and has similarity to E. coli L6 and rat L9 ribosomal proteins }\end{array}$ \\
\hline YDL061C & RPS29B & Dn & -3.54772 & 8 & $\begin{array}{l}\text { Protein component of the small (40S) ribosomal subunit; nearly identical to } \\
\text { Rps29Ap and has similarity to rat S29 and E. coli S14 ribosomal proteins }\end{array}$ \\
\hline YFR040W & SAP155 & Dn & -3.72949 & 3 & $\begin{array}{l}\text { Protein that forms a complex with the Sit } 4 p \text { protein phosphatase and is required } \\
\text { for its function; member of a family of similar proteins including Sap4p, Sap185p, } \\
\text { and Sap190p }\end{array}$ \\
\hline YBL102W & SFT2 & Up & -3.82416 & 6 & $\begin{array}{l}\text { Non-essential tetra-spanning membrane protein found mostly in the late Golgi, can } \\
\text { suppress some sed5 alleles; may be part of the transport machinery, but precise } \\
\text { function is unknown; similar to mammalian syntaxin } 5\end{array}$ \\
\hline YBL102W & SFT2 & Dn & -3.33554 & $"$ & " \\
\hline YOR137C & SIA1 & Up & -3.12923 & 12 & $\begin{array}{l}\text { Protein of unassigned function involved in activation of the Pma1p plasma } \\
\text { membrane H+-ATPase by glucose }\end{array}$ \\
\hline YOR137C & SIA1 & Dn & -3.03827 & " & " \\
\hline YDL033C & SLM3 & Dn & -3.17629 & 1 & $\begin{array}{l}\text { tRNA-specific 2-thiouridylase, responsible for 2-thiolation of the wobble base of } \\
\text { mitochondrial tRNAs; human ortholog is implicated in myoclonus epilepsy } \\
\text { associated with ragged red fibers (MERRF) }\end{array}$ \\
\hline YHR030C & SLT2 & $\mathrm{Dn}$ & -3.64054 & 3 & $\begin{array}{l}\text { Serine/threonine MAP kinase; involved in regulating maintenance of cell wall } \\
\text { integrity, progression through the cell cycle, and nuclear mRNA retention in heat } \\
\text { shock; required for mitophagy and pexophagy; affects recruitment of mitochondria } \\
\text { to the phagophore assembly site (PAS); regulated by the PKC1-mediated signaling }\end{array}$ \\
\hline
\end{tabular}


pathway

\begin{tabular}{|c|c|c|c|c|c|}
\hline YBR172C & SMY2 & $\mathrm{Dn}$ & -3.55944 & 12 & $\begin{array}{l}\text { Protein of unknown function involved in COPII vesicle formation; interacts with the } \\
\text { Sec23p/Sec } 24 p \text { subcomplex; overexpression suppresses the temperature } \\
\text { sensitivity of a myo2 mutant; has similarity to S. pombe Mpd2 }\end{array}$ \\
\hline YBR172C & SMY2 & Up & -3.22684 & " & " \\
\hline YLR025W & SNF7 & Dn & -3.44354 & 6 & $\begin{array}{l}\text { One of four subunits of the endosomal sorting complex required for transport III } \\
\text { (ESCRT-III); involved in the sorting of transmembrane proteins into the } \\
\text { multivesicular body (MVB) pathway; recruited from the cytoplasm to endosomal } \\
\text { membranes }\end{array}$ \\
\hline YLR025W & SNF7 & Up & -3.12536 & " & " \\
\hline YHR163W & SOL3 & $\mathrm{Dn}$ & -4.0004 & 12 & $\begin{array}{l}\text { 6-phosphogluconolactonase, catalyzes the second step of the pentose phosphate } \\
\text { pathway; weak multicopy suppressor of los } 1-1 \text { mutation; homologous to Sol } 2 \mathrm{p} \text { and } \\
\text { Sol1p }\end{array}$ \\
\hline YBR169C & SSE2 & Dn & -4.28149 & 12 & $\begin{array}{l}\text { Member of the heat shock protein } 70 \text { (HSP70) family; may be involved in protein } \\
\text { folding; localized to the cytoplasm; highly homologous to the heat shock protein } \\
\text { Sse1p }\end{array}$ \\
\hline YDR395W & SXM1 & Dn & -4.20958 & 6 & $\begin{array}{l}\text { Nuclear transport factor (karyopherin) involved in protein transport between the } \\
\text { cytoplasm and nucleoplasm; similar to Nmd5p, Cse1p, Lph2p, and the human } \\
\text { cellular apoptosis susceptibility protein, CAS1 }\end{array}$ \\
\hline YDR395W & SXM1 & Up & -4.11698 & " & " \\
\hline YML072C & TCB3 & Up & -3.30324 & 1 & $\begin{array}{l}\text { Lipid-binding protein, localized to the bud via specific mRNA transport; non-tagged } \\
\text { protein detected in a phosphorylated state in mitochondria; GFP-fusion protein } \\
\text { localizes to the cell periphery; } C \text {-termini of } T c b 1 p, T c b 2 p \text { and Tcb3p interact }\end{array}$ \\
\hline YJL138C & TIF2 & Up & -3.35019 & 12 & $\begin{array}{l}\text { Translation initiation factor elF4A, identical to Tif1p; DEA(D/H)-box RNA helicase } \\
\text { that couples ATPase activity to RNA binding and unwinding; forms a dumbbell } \\
\text { structure of two compact domains connected by a linker; interacts with elF4G }\end{array}$ \\
\hline YPR074C & TKL1 & $\mathrm{Dn}$ & -3.36535 & 12 & $\begin{array}{l}\text { Transketolase, similar to TkI2p; catalyzes conversion of xylulose-5-phosphate and } \\
\text { ribose-5-phosphate to sedoheptulose-7-phosphate and glyceraldehyde-3- } \\
\text { phosphate in the pentose phosphate pathway; needed for synthesis of aromatic } \\
\text { amino acids }\end{array}$ \\
\hline YNL070W & TOM7 & Dn & -3.22655 & 1 & $\begin{array}{l}\text { Component of the TOM (translocase of outer membrane) complex responsible for } \\
\text { recognition and initial import steps for all mitochondrially directed proteins; } \\
\text { promotes assembly and stability of the TOM complex }\end{array}$ \\
\hline YML028W & TSA1 & Up & -3.40694 & 12 & $\begin{array}{l}\text { Thioredoxin peroxidase, acts as both a ribosome-associated and free cytoplasmic } \\
\text { antioxidant; self-associates to form a high-molecular weight chaperone complex } \\
\text { under oxidative stress; deletion results in mutator phenotype }\end{array}$ \\
\hline YER151C & UBP3 & Dn & -3.05987 & 6 & $\begin{array}{l}\text { Ubiquitin-specific protease that interacts with Bre5p to co-regulate anterograde } \\
\text { and retrograde transport between the ER and Golgi; inhibitor of gene silencing; } \\
\text { cleaves ubiquitin fusions but not polyubiquitin; also has mRNA binding activity }\end{array}$ \\
\hline YDR049W & VMS1 & Up & -3.01592 & 12 & $\begin{array}{l}\text { Component of a Cdc48p-complex involved in protein quality control; exhibits } \\
\text { cytosolic and ER-membrane localization, with Cdc48p, during normal growth, and } \\
\text { contributes to ER-associated degradation (ERAD) of specific substrates at a step } \\
\text { after their ubiquitination; forms a mitochondrially-associated complex with Cdc48p } \\
\text { and Npl4p under oxidative stress that is required for ubiquitin-mediated } \\
\text { mitochondria-associated protein degradation (MAD); conserved in C. elegans and } \\
\text { humans }\end{array}$ \\
\hline YDR049W & VMS1 & $\mathrm{Dn}$ & -3.00464 & $"$ & " \\
\hline YDR431W & - & $\mathrm{Dn}$ & -5.47989 & 12 & $\begin{array}{l}\text { Dubious ORF unlikely to encode a functional protein, based on available } \\
\text { experimental and comparative sequence data }\end{array}$ \\
\hline YDR521W & - & Dn & -3.85005 & 12 & $\begin{array}{l}\text { Dubious ORF that overlaps YDR520C; mutant increases expression of PIS1 and RPL3 } \\
\text { in glycerol }\end{array}$ \\
\hline YDR521W & - & Up & -3.22263 & " & " \\
\hline YEL007W & - & $\mathrm{Dn}$ & -3.43104 & 12 & $\begin{array}{l}\text { Putative protein with sequence similarity to S. pombe gti1+ (gluconate transport } \\
\text { inducer 1) }\end{array}$ \\
\hline YER187W & - & Up & -3.61922 & 12 & Putative protein of unknown function; induced in respiratory-deficient cells \\
\hline YER187W & - & Dn & -3.47411 & " & " \\
\hline YGL042C & - & Dn & -4.36732 & 12 & $\begin{array}{l}\text { Dubious open reading frame, not conserved in closely related Saccharomyces } \\
\text { species; deletion mutation blocks replication of Brome mosaic virus in S. cerevisiae, } \\
\text { but this is likely due to effects on the overlapping gene DST1 }\end{array}$ \\
\hline YGL072C & - & Dn & -3.72356 & 12 & $\begin{array}{l}\text { Dubious open reading frame unlikely to encode a protein; partially overlaps the } \\
\text { verified gene } \mathrm{HSF} 1 \text {; null mutant displays increased resistance to antifungal agents } \\
\text { gliotoxin, cycloheximide and } \mathrm{H} 2 \mathrm{O} 2\end{array}$ \\
\hline YIL100W & - & Dn & -3.34288 & 12 & $\begin{array}{l}\text { Dubious open reading frame unlikely to encode a protein, based on available } \\
\text { experimental and comparative sequence data; completely overlaps the dubious }\end{array}$ \\
\hline
\end{tabular}




\begin{tabular}{|c|c|c|c|c|c|}
\hline & & & & & ORF YIL100C-A \\
\hline YLL044W & - & Up & -3.43439 & 12 & $\begin{array}{l}\text { Dubious open reading frame unlikely to encode a protein, based on available } \\
\text { experimental and comparative sequence data; transcription of both YLLO44W and } \\
\text { the overlapping gene RPL8B is reduced in the gcr1 null mutant }\end{array}$ \\
\hline YLR241W & - & Dn & -3.03164 & 12 & Putative protein of unknown function, may be involved in detoxification \\
\hline YDR057W & YOS9 & Up & -3.03355 & 11 & $\begin{array}{l}\text { ER quality-control lectin; integral subunit of the HRD ligase; binds to glycans with } \\
\text { terminal alpha- } 1,6 \text { linked mannose on misfolded N-glycosylated proteins and } \\
\text { participates in targeting proteins to ERAD; member of the OS-9 protein family }\end{array}$ \\
\hline
\end{tabular}

Table 3.2 List of hypersensitive mutants derived from HOP screen of FC-888 treated cells. 94 tags were identified that exceeded the threshold of three standard deviations from the mean. These tags represent 74 unique genes with 20 represented by both Up and Dn tags. Genes were grouped according to cellular component/process: Group 1: Mitochondria; 2: Apoptosis; 3: Phosphorylation; 4: Nucleus; 5: Cell cycle; 6: Transport; 7: Catabolism; 8: Ribosome; 9: Transcription; 10: Protein Synthesis; 11: ER/Golgi; 12: Miscellaneous or putative.

After processing and removal of genes implicated in multidrug resistance, 41 genes were identified as hypersensitive in the FC-592 treated HOP screen. Of these 12 genes $(29.3 \%)$ were identified as involved in protein production through the ER/Golgi/Glycosylation. These data could indicate a link between FC-592's mode of action and protein synthesis disruption. 10 genes (24.4\%) were identified as being involved in transcription although this is a very large class of genes, and not necessarily representative of enrichment.

The HOP screen of FC-888 treated cells revealed 74 hypersensitive deletion mutants. The largest class represented was transport associated proteins ( 9 genes, $12.2 \%$ ). Again, this is a very large class of genes, and does not necessarily represent significant enrichment. Genes representing hits in this screen were distributed over a large range of cellular components and processes, including many for which there was but a single representative. 29 genes from this set were designated as being of putative or unknown function, or having a function unrelated to any others within the set. These genes are regarded as unlikely as having a functional role in FC-888 sensitivity or representing functional enrichment.

For both screens, statistical analysis was used to determine the relevance of Gene Ontology enrichment within these data sets. 


\subsubsection{Gene Ontology (GO) Term analysis}

The Biological Networks Gene Ontology tool (BiNGO; http://www.psb.ugent.be/cbd/papers/BiNGO/) was used to determine which Gene Ontology (GO) terms are significantly overrepresented in the set of genes represented by the hits in the FC-592 and FC-888 HOP screens. For this analysis, genes within the HOP screen sets were compared with genes from the yeast genome as a whole. Because essential genes and genes implicated in multi-drug resistance by Hillenmeyer et al. (2008) were removed from the HOP screens, these genes were also disregarded from the BiNGO analysis.

No significant enrichment was found at the $1 \%$ confidence level for genes in the FC- 888 HOP screen. This means that the hits from this screen were distributed among the components and processes of the genome in a largely random fashion. This means there is no evidence that FC-888 affects any single pathway or function within the cell in a specific way that would make mutants deficient in that

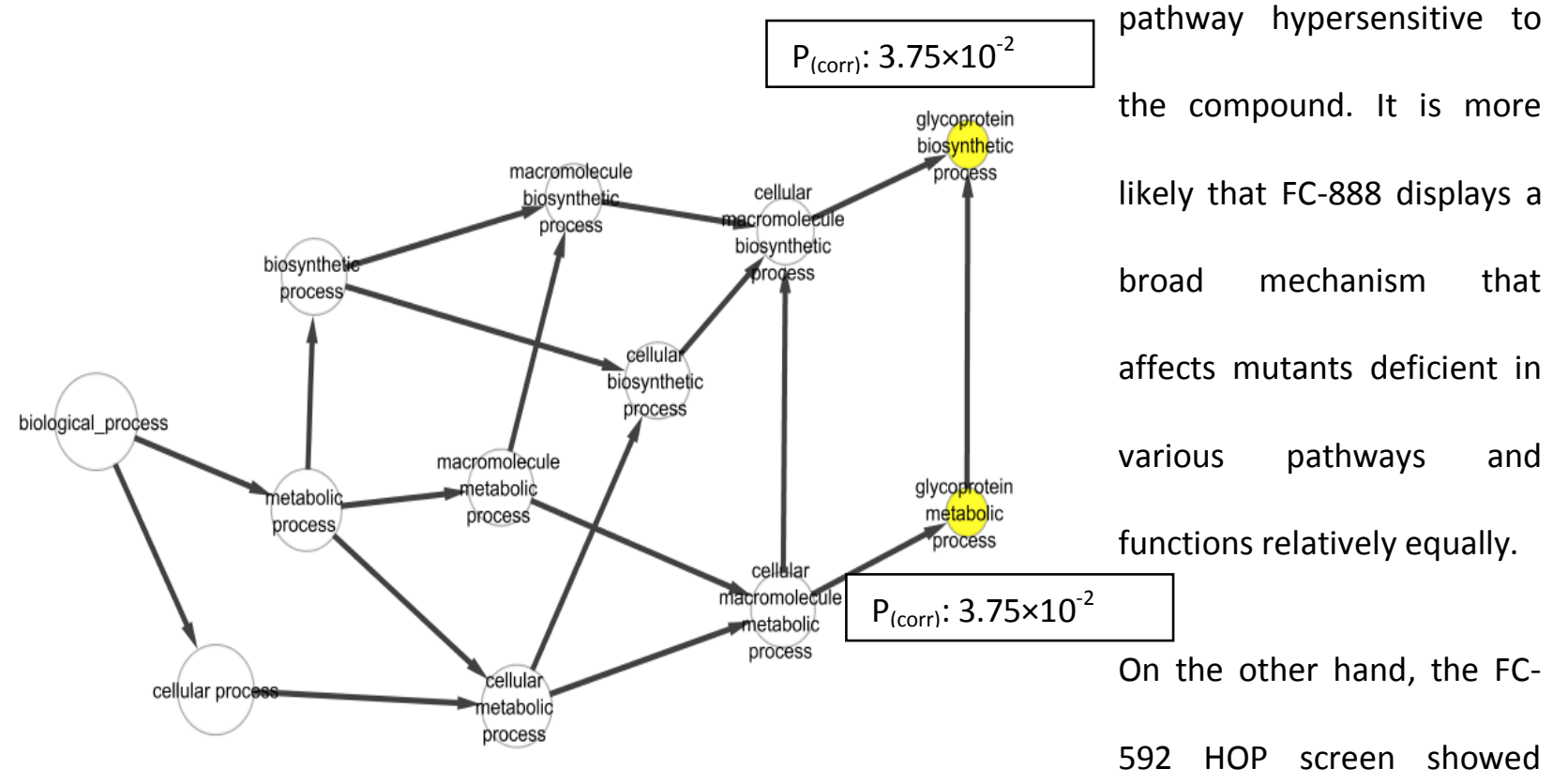

Figure 3.4 Genetic networks enriched for genes linked to FC-592 sensitivity. Glycoprotein Biosynthetic processes and metabolism are linked to further networks, including the secretory pathway.

significant enrichment in glycoprotein biosynthetic processes and glycoprotein metabolic processes at the $1 \%$ confidence level. These processes are based on the genes ALG6, ALG8, GDA1, HOC1 and OST6 which appear in the HOP screen set. ALG6, 
ALG8 and GDA1 occur within the set as both Up and Dn tags, providing extra evidence for their relevance.

\subsubsection{Hit confirmation for FC-592 HOP screen}

23 strains recording hits in the FC-592 HOP assay were screened to confirm sensitivity to the compound in comparison to a wild type train (Y7092). This limited selection included all hits recorded that were grouped into ER/Golgi/glycoylation genes related to protein synthesis and transport; as well as transcription related genes; the G2/M phase progression protein PIN4; the fatty acid elongase FEN1; and the NAP family histone chaperone gene VPS75. These selections were made because of the limited quantity of compound available at this stage in the experimental process and their relevance to predicting a specific mechanism. In short, these genes were the only ones related enough to provide evidence for FC-592 action on a specific process. PIN4 and FEN1 were included because both Up and Dn tags showed up as hits in the HOP screen, increasing their relevance. For a similar reason, confirmation screens of FC-888 hits were deemed unnecessary at this point: no collection of genes from within that set offer evidence for a specific mechanism. 


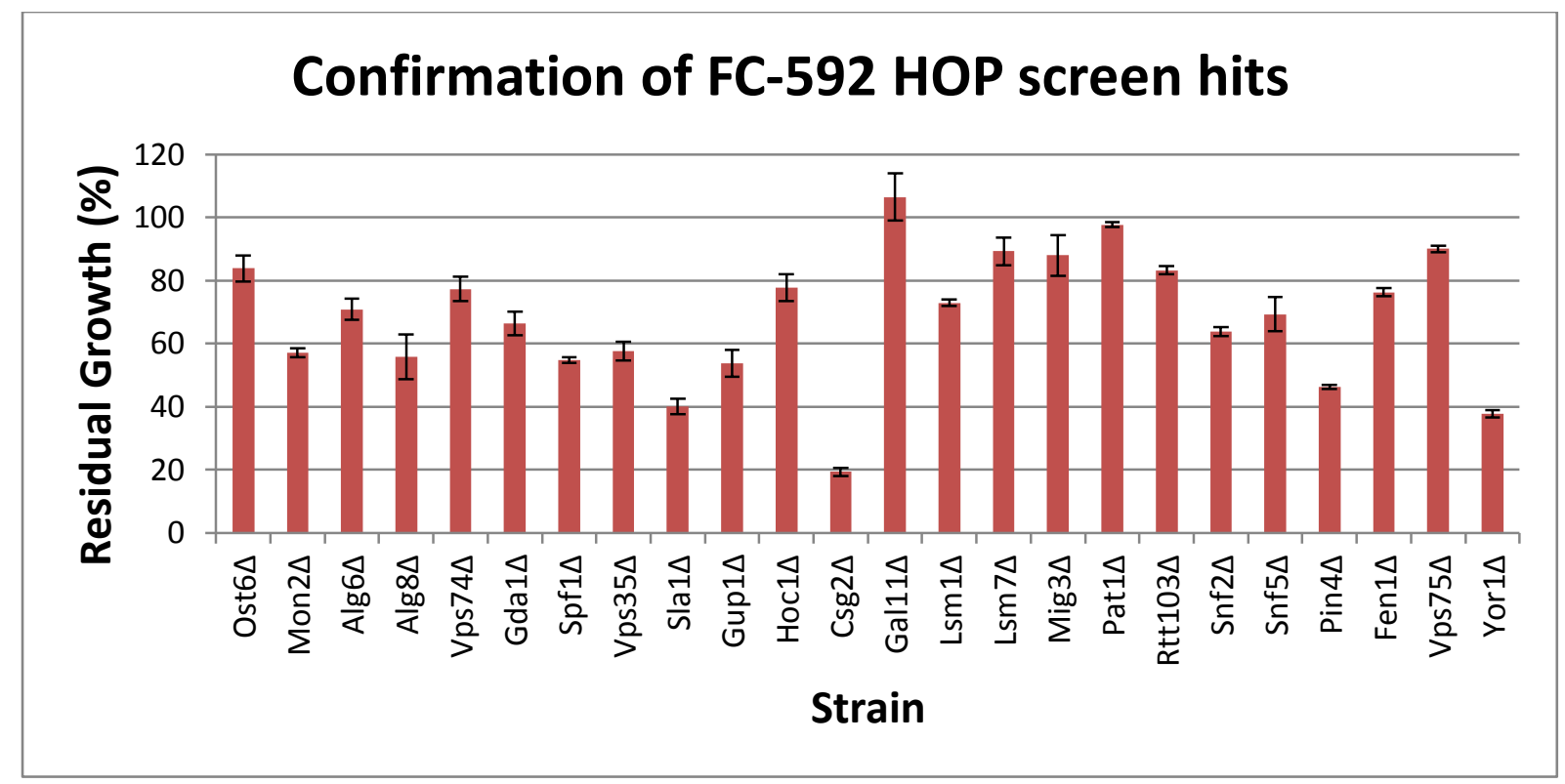

Figure 3.6 Confirmation screen for FC-592 HOP assay hits. All of the tested strains confirmed some level of additional sensitivity to FC-592 relative to a WT control strain ( $<90 \%$ residual growth), apart from the RNA polymerase II mediator complex subunit protein GAL11 and the mRNA-decapping factor PAT1.

\subsubsection{Unfolded protein response assay}

In order to investigate whether the unfolded protein response (UPR) was activated by FC-592 treatment, YCG266-4×UPRE-GFP yeast cells were treated with the compound and examined for UPR expression via confocal microscopy. In this reporter strain, induction of the UPR causes increased GFP expression, which is measured as a stronger signal at $520 \mathrm{~nm}$. FC-592 treated cells were compared to cells treated with the known UPR inducer Dithiothreitol (DTT).

\begin{tabular}{|rr|rr|}
$\begin{array}{l}\text { DTT } \\
(\mathrm{mM})\end{array}$ & \multicolumn{1}{l}{$\begin{array}{l}\text { Whole Cell GFP } \\
\text { intensity }\end{array}$} & \multicolumn{1}{c|}{$\begin{array}{l}\text { FC-592 } \\
(\boldsymbol{\mu M})\end{array}$} & $\begin{array}{l}\text { Whole Cell GFP } \\
\text { intensity }\end{array}$ \\
\hline $\mathbf{0}$ & 353.428 & 0 & 340.081 \\
\hline $\mathbf{0 . 1 2 5}$ & 462.809 & 1.75 & 337.232 \\
\hline $\mathbf{0 . 2 5}$ & 683.612 & 3.5 & 353.869 \\
\hline $\mathbf{0 . 5}$ & 1013.08 & 7 & 334.125 \\
\hline $\mathbf{1}$ & 1112.16 & 14 & 349.495 \\
\hline $\mathbf{2}$ & 1396.02 & 28 & 385.384 \\
\hline $\mathbf{4}$ & 1442.35 & 56 & 365.244 \\
\hline $\mathbf{8}$ & 1524.05 & 112 & 388.802 \\
\hline
\end{tabular}

Table 3.3 Induction of UPR is not observed in FC-592 treated cells. Whole cell GFP intensity, measured at $520 \mathrm{~nm}$, increases with concentration in DTT treated cells, indicating a dose dependant response. GFP intensity remains relatively constant in FC-592 treated cells, indicating an extremely weak or non-existent response. 
DTT treated YCG326 cells

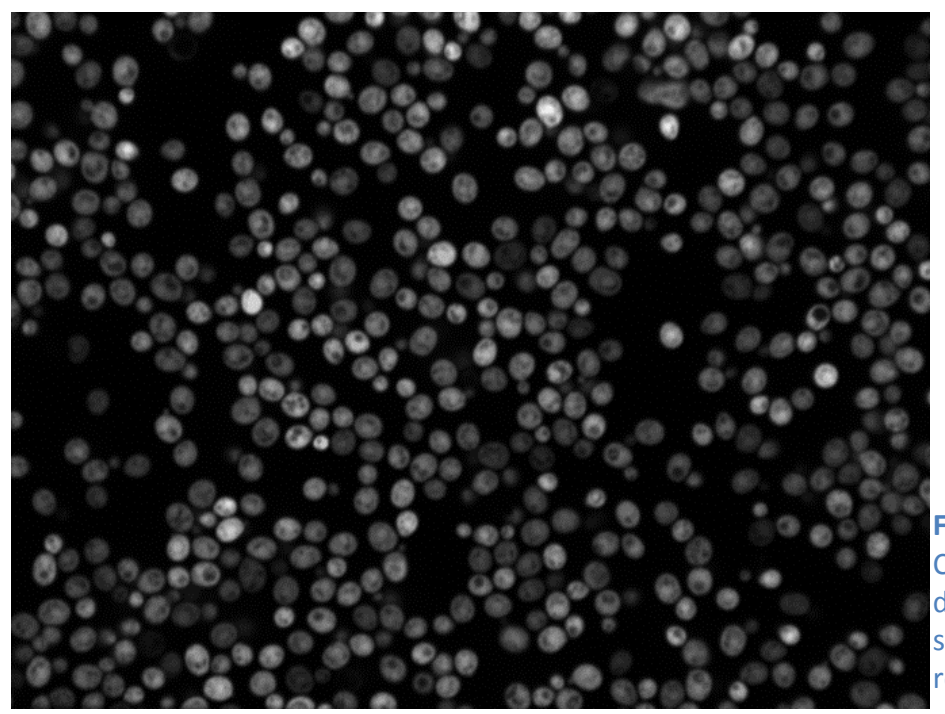

Figure 3.7 YCG326 cells treated for $4 \mathrm{~h}$ with $8 \mathrm{mM}$ DTT. Cells were examined using confocal microscopy to determine induction of the UPR. The increased GFP signal intensity measured at $520 \mathrm{~nm}$ indicates a strong response to treatment with this compound.

FC-592 Treated YCG326 cells

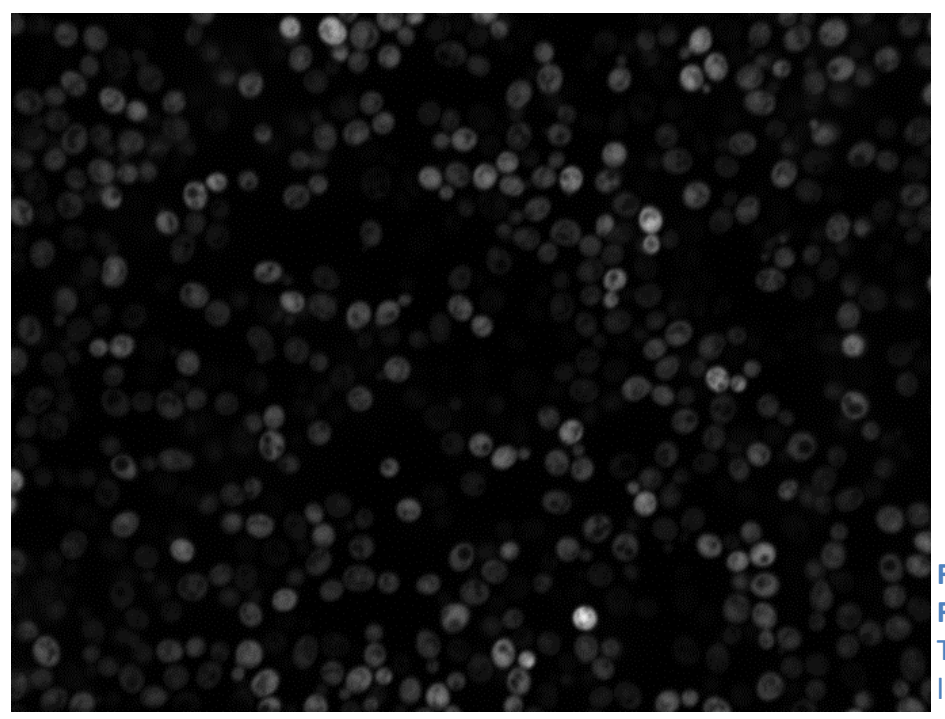

Figure 3.8 YCG326 cells treated for $4 \mathrm{~h}$ with $112 \mu \mathrm{M}$ FC-592. No evidence is seen for induction of the UPR. The GFP signal intensity measured at $520 \mathrm{~nm}$ is much lower than that seen for DTT treated cells.

No evidence was seen for induction of the UPR by treatment with FC-592 in this assay. This means that the glycosylation biosynthetic processes suggested by the HOP assay (and which may have caused misfolded proteins upon disruption) cannot be confirmed as a target for FC-592. However, this result does not disprove any effect on this process, at least as a partial target of the compound. Absence of UPR does not mean that glycosylation processing genes, as distinct from glycosylation synthetic genes or genes affected by glycosylation post-ER are not involved. Glycoprotein synthesis is 
a common process (Murray et al. 2006) throughout the cell, and disruption may have more subtle effects, not triggering the UPR. 


\subsection{Discussion}

\subsubsection{Chemigenomic HOP screen of FC-592}

A chemigenomic screen using yeast tag microarray technology was conducted using cells treated with FC-592 and FC-888 in an attempt to determine a likely target process or pathway for each of these inhibitory compounds. The homozygous profiling (HOP) screen takes advantage of modern advances in yeast genomics to identify single gene deletion strains which are hypersensitive to a certain compound (Nislow \& Giaever, 2007). This is possible through the use of libraries of viable single gene deletion mutants encompassing some $\sim 80 \%$ of the genome, in this case consisting of homozygous diploid cells. The deletion strains, whilst viable, are generally sensitive to attack in the pathways and processes for which they possess a null mutation. Identifying sensitive strains therefore gives insight into the likely mechanism of the screened compounds.

The two screens were treated with a sub-inhibitory dose of the compounds and compared via tag microarray to a DMSO treated control. Tests conducted to identify an appropriate dose for these experiments revealed that the diploid cells used in these experiments were slightly less sensitive to FC-592 and FC-888 than haploid cells used in earlier inhibition screens. This is likely because diploid cells are larger than their haploid counterparts (Herskowitz, 1988) and thus have a lower surface area to volume ratio. For any compound dispersing across the membrane as a means of entry to the cell, this means that cytosolic concentration is likely to be marginally lower in larger cell types such as diploid cells. Additionally, diploid cells possess two copies of every gene and with a higher gene copy number (Gruber et al. 2012) may be slightly more tolerant where gene dosage plays a factor.

Deletion mutants (and the genes they represent) were counted as 'hits' if their z-score (standard deviations below the mean) was at least -3.0 in the treated cell populations, compared to the untreated controls. The three standard deviation cut-off for significance was an arbitrary threshold that represents just a $0.13 \%$ chance of a gene appearing as a hit by chance alone, assuming underlying normality. The HOP screens identified 41 genes as 'hits' in the FC-592 treated cell 
population, and 74 genes as 'hits' in the FC-888 treated cells. Of these, 7 genes in FC-592 and 20 genes in FC-888 were identified by both Up and Dn tags, making them especially relevant as hypersensitive mutants.

Whilst many genes were identified as mediating sensitivity to FC-592 and FC-888, the evidence is less clear cut supporting a particular target pathway or process. Gene enrichment was examined using the Biological Network Gene Ontology (BiNGO) tool (Maere, 2005). No significant enrichment was found for FC-888, whilst glycosylation biosynthetic and metabolic processes were identified as enriched in the FC-592 sensitive strains. Statistical evaluation therefore gave only limited insight into possible mode-of-action for these compounds. Chemigenomic analysis studies typically show enrichment in several components or processes, pointing to a larger pathway (Blackman et al. 2012).

Examining the implications of glycosylation biosynthetic and metabolic processes as a sensitizer for FC-592, several determinations can be made. Biological glycosylation reactions involve the attachment of glycan carbohydrates to proteins and lipids to serve a variety of functions (Varki, 2009). This process is important for correct folding of certain proteins, and in the secretory pathway where polysaccharides linked at the amide nitrogen of asparagine in the protein confer stability on some secreted glycoproteins. Glycosylation also helps mediate cell-cell adhesion through sugarbinding lectin proteins. These mediate specific cell-cell interactions through recognition of certain carbohydrate moieties. In addition, experiments have shown that glycosylation is not a strict requirement for proper folding, but incorrectly glycosylated or non-glycosylated proteins are recognised as such by cellular mechanisms and quickly degraded (Parodi, 2000).

FC-592 is implicated in affecting glycosylation biosynthetic and metabolic processes because of the genes ALG6, ALG8, OST6, GDA1 and HOC1, which are associated with the gene ontology (GO) functional terms $\mathrm{N}$-directed glycosylation/deglycosylation; peptidoglycan anabolism; and modification with sugar residues (Mewes et al. 2002). In addition VPS74, VPS35, MON2 and GUP1 were associated with functional terms linked with vacuolar/lysosomal transport and carbohydrate 
transport. These all have links to the ER and secretory pathway, suggesting some activity in this functional area. The gene hits recorded do not represent a comprehensive interaction with genes of the secretory pathway. The lack of secretory pathway genes such as CPR8 (Dolinski et al. 1997); KEX2 (Fuller et al. 1989); SPC2 (Fang et al. 1997) and LST4 (Roburg et al. 1997), amongst the sensitive mutant set means that the case for a direct mode-of-action on the sectretory pathway is not definitive. However it is a good indication of probable activity in this area.

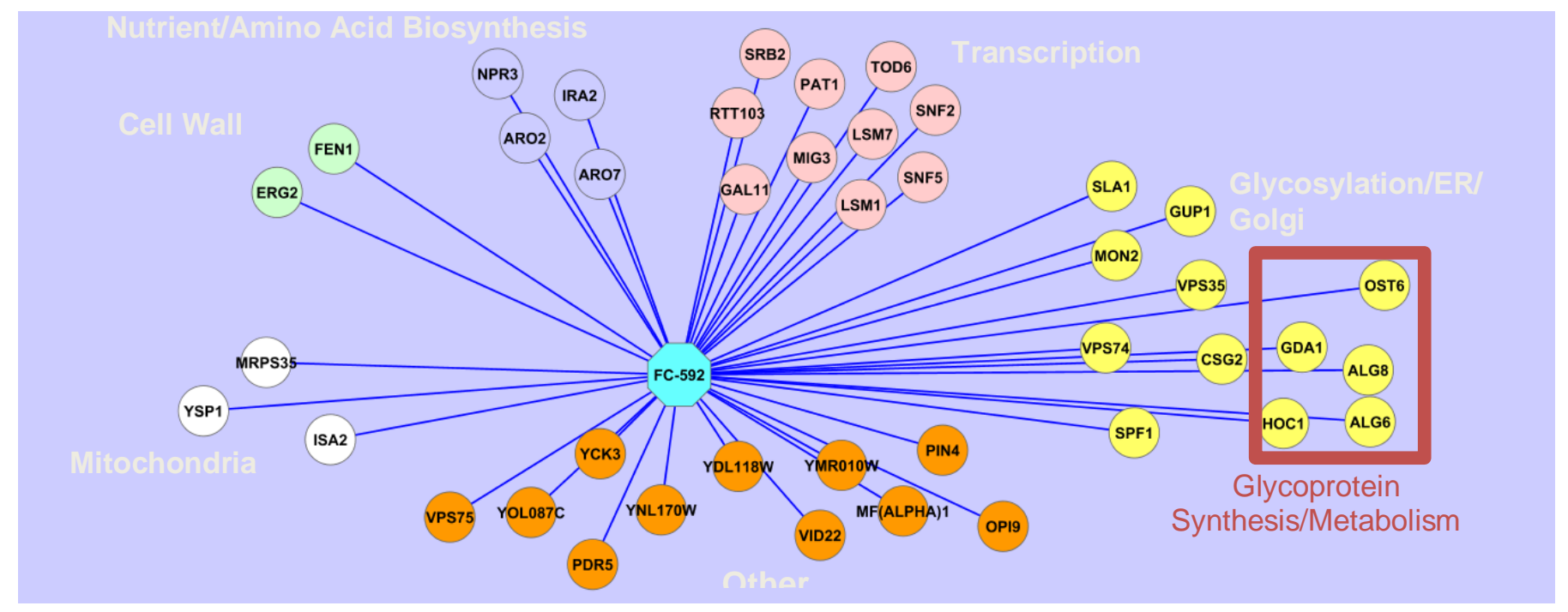

Figure 3.9 Cluster analysis of FC-592 hits. Genes involved with glycosylation and located to the Endoplasmic Reticulum and Golgi make up the largest group. These genes are linked to the secretory pathway. Transcription related genes also feature strongly, although this is a large group and no statistical enrichment was seen in this group. A number of miscellaneous genes unrelated to others in this group were labelled as other; this group includes one of the most intriguing hits on this list: PIN4.

Whilst statistical analysis of hits did not give conclusive evidence of a mode-of-action for FC-592, some insight can still be gained from examining the individual genes implicated in FC-592 resistance. Many Secretory pathway linked genes were not observed in the screen. However, the secretory pathway in yeast has at least 173 associated genes (Hartman et al. 2001). This means that there are probably redundant networks as distinct from redundant pathways within networks. Thus lack of a Synthetic Lethal (SL) mutant-drug pair in the HOP screen might only mean another network has taken over the function. This possibility allows assessment of a hit but not the discounting of a process by lack of a hit. 
Of the 41 sensitive strains identified in the HOP assay, seven were represented by both Up and Dn tags. These were ALG6, ALG8, FEN1, GDA1, MON2, PIN4 and SNF2. ALG6, ALG8, GDA1 and MON2 are associated with the above mentioned glycosylation and ER linked transport. The fact that these genes presented with both tag types reinforces their relevance. There are numerous reasons why a tag might be filtered out from the yeast tag microarray dataset, such as poor signal quality. However, the presence of both tags inspires confidence that these results were not due to random variance or some microarray based statistical artefact: the mutants represented by these genes were certainly sensitive in the experimental sample. Each of these hits was confirmed as sensitive on a follow up dose response assay.

ALG6 and ALG8 are glucosyltransferases, directly involved in glycosylation (Reiss et al. 1996; Runge \& Robbins, 1986). They appear to be involved in mediating resistance to a number of compounds, including hygromycin B and neothyonidiocide (Dudley et al. 2005; Yibmantasiri et al. 2012). Therefore whilst these genes likely have relevance to the general mechanism of FC-592 activity, they are unlikely to point to a direct target. The guanosine diphosphatase GDA1 is located in the Golgi and mediates transport of GDP-mannose into the Golgi lumen (Beninsone et al. 1994). It too is implicated in mediation of resistance to a number of compounds. These tend to be protein synthesis inhibitors such as cycloheximide, tunicamycin and maramomycin however (Alamgir et al. 2010; Kapitzky et al. 2010). A common mechanism of action, the presence of this gene in the FC-592 dataset is potentially an excellent indicator of the compound's mode-of-action. It is however by no means definitive: there are many pathways linked with protein synthesis that could have downstream effects on a gda $1 \Delta$ mutant.

MON2, a membrane protein encoding gene associated with endocytosis and vacuole integrity, is involved with the Sec7p family of proteins (Jochum et al. 2002). This is closely linked with the secretory pathway, but again is involved with mediating resistance to a range of drugs such as the TORC1 pathway inhibitor rapamycin (Xie et al. 2005) and the ergosterol biosynthesis inhibitor 
fenpropimorph (Kapitzky et al. 2010). The varied nature of the compounds to which MON2 mediates resistance suggests a general fitness response rather than any specific pathway which might illuminate a target for FC-592. However it is illustrative that the compound is associated so closely with the sectretory pathway: a mechanism linked with other FC-592 sensitive mutants. In this way a group of genes provides evidence in a certain direction, where any single gene on its own could be ignored as frivolous.

In a similar manner, the fatty acid elongase FEN1 is involved in sphingolipid biosynthesis and has regulatory effects on 1,3-beta-glucan synthase, vacuolar ATPase, and the secretory pathway (David et al. 1998). Like MON2, it seems to implicated in general toxicity resistance, mediating both resistance (rapamycin, bleomycin, amioderone, etc.) and sensitivity (fluconazole, hydroxyurea, etc.) (Alamgir et al. 2010; Kapitzky et al. 2010; Xie et al. 2005; Yadav et al. 2007). Some compounds, such as cycloheximide, register sensitivity or resistance mediated by FEN1 depending on the screen (Alamgir et al. 2010; Kapitzky et al. 2010)! It is therefore clear that FEN1 cannot prove a target pathway for FC-592, but the proximity to the secretory pathway is again intriguing.

The SWI/SNF chromatin remodelling complex associated gene SNF2 (Peterson et al. 1995) is not associated with the mechanisms mentioned above. It mediates resistance to a very large array of anti-proliferatives (Alamgir et al. 2010; Lawrence et al. 2004). In addition, snf2 $\Delta$ mutants are defective in sporulation, growth on nonfermentable carbon sources, mating-type switching and display increased sensitivity to mutagenising agents (Dror \& Winston, 2004; Stern et al. 1984; Carlson et al. 1981; Backer \& Foury, 1985). This data suggests that SNF2 mediates general cell fitness rather than resistance to attack on a certain pathway and is unlikely to offer insight into targets for FC-592.

Of greater interest is the $G_{2} / M$ phase progression protein-encoding gene PIN4 (Pike et al. 2004). The second checkpoint in the cell cycle, this checkpoint triggers the start of the mitotic phase. If all the conditions required to ensure the cell is ready for mitosis are met, the cell initiates the signalling 
cascade that signals the beginning of mitosis (Cooper, 2000). This is of interest because some of the most important anti-cancer drugs such as paclitaxel cause cell cycle arrest at the $G_{2} / M$ checkpoint (Bharadwaj \& Yu, 2004). In addition, further testing revealed that FC-592 causes a block at the $G_{2} / M$ checkpoint, and thus this gene has probable relevance to the mode-of-action. This is discussed in further detail in chapter 4 .

Other significant hits from the set linked to glycoprotein synthesis or the secretory pathway include the ER membrane mannosylation mediating protein Csg2p (Tanida et al. 1996); the chorismate mutase ARO7 (Ball et al. 1986); and the chorismate synthase ARO2 (Jones et al. 1991). Taken together with the previously described glycoprotein synthesis/secretory pathway linked genes GDA1, ALG6, MON2, FEN1 and HOC1 these represent 8 of the top 12 hits from the HOP screen, in terms of z-score.

This is of course a very wide ranging pathway encompassing a lot of genes within the genome. It is also true that FC-592 is likely to be a non-specific binder of hydrophobic pockets (see Chapter 2), a target that appears all over the cell. However it is still a good indication that these processes are affected by the compound. It is probable that FC592 has weak activity against a lot of process, but perhaps a bit more strongly against glycoprotein biosynthesis and the secretory pathway.

\subsubsection{Chemigenomic HOP screen of FC-888}

74 genes were identified as mediating sensitivity to FC-888 in the HOP screen for this compound. Unlike FC-592, the hits in this dataset represented no statistical enrichment for any GO term component, function or process. Therefore, it is difficult to build a case for the activity of FC-888 against any single target process or pathway from this evidence. Considering the analysis of FC-888's chemistry in chapter 2 as likely being a non-specific alkylating agent, this result is unsurprising: A range of targets all across the cell were affected and null mutants representing genes linked to many of those targets mediated sensitivity to the compound. 


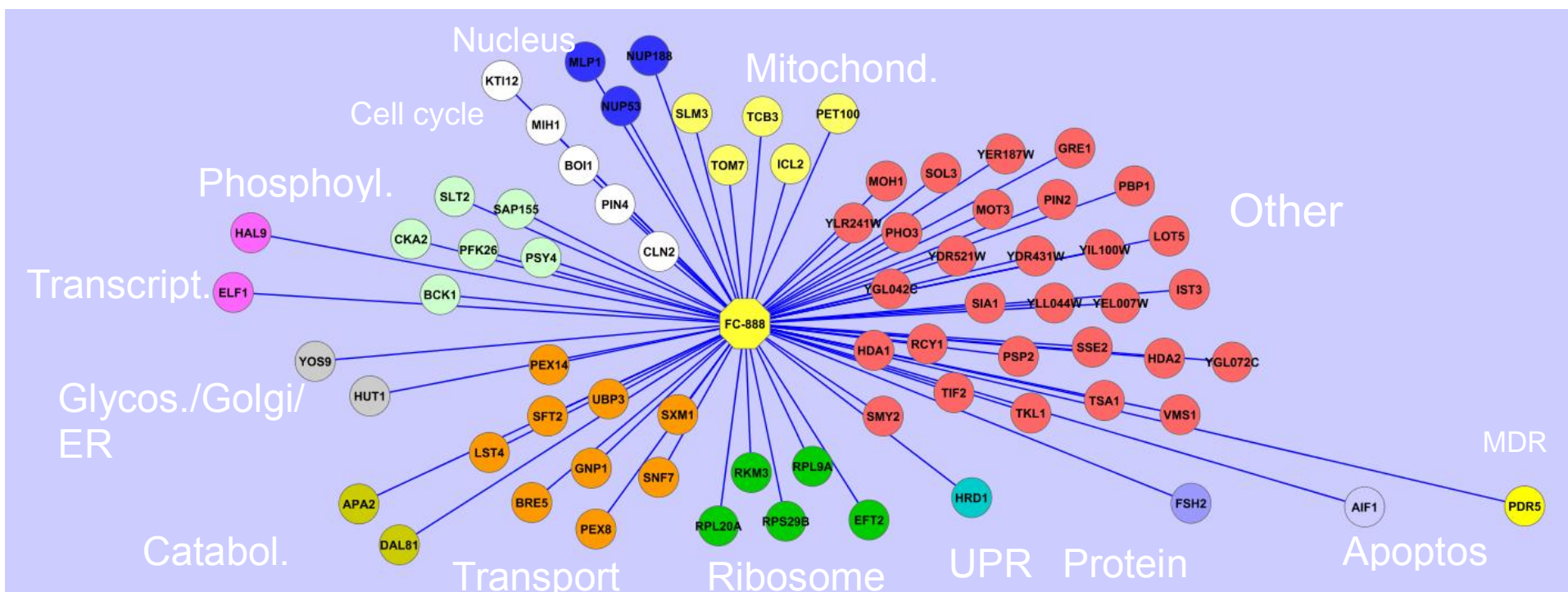

Figure 3.10 Cluster analysis of FC-888 hits. A very wide range of genes are represented by hits in the FC- 888 HOP screen. No single category is overrepresented to such an extent as to imply a potential target process or pathway for the compound. When considering the likely function of FC888 as a non-specific alkylating agent, this makes sense.

20 genes from the HOP screen were represented by both Up and Dn tags. For reasons discussed above, these are likely to be particularly relevant hits; there is less chance of them appearing in the dataset as a result of some statistical artefact or experimental variation. Genes represented by these hits include APA2, BCK1, DAL81, ELF1, FSH2, GRE1, ICL2, LOT5, LST4, MOT3, PBP1, PEX14, PHO3, RCY1, SFT2, SIA1, SMY2, SNF7, SXM1 and VMS1. These range in function from the methylisocitrate lyase protein of the mitochondrial matrix ICL2, which functions in glycolysis (Luttik et al. 2000), to the nuclear transcription factor MOT3, to the ER-associted degradation protein VMS1, which forms complexes with Cdc48p and functions at both the ER and mitochondria (Tran et al. 2011). There is no pattern to these genes which points to or suggests a cellular target process, component or function.

Conspicuous by their absence are any genes associated with DNA repair, such as RAD1, RAD10, MGT1, SRS2 and DIN7 (Xaio et al. 1991; Prakash et al. 1993). With the strong likelihood that FC-888 is an alkylating agent, it would be expected to find some of these genes amongst the HOP screen dataset. Apart from pointing to the fact that FC-888 likely targets a host of cellular nucleophiles independent of DNA, this screen doesn't reveal a great deal about the compound or its targets. 
There is a good chance that much of the data from this experiment is clouded by background 'noise' and experimental variation. A single run of the experiment may not be reliable enough to make solid conclusions.

\subsubsection{Assessment of yeast tag microarray HOP screen as an experimental method}

Yeast tag microarrays are a proven technology with an established track record in identifying the targets of small molecule bioactives (Singh-Babak et al. 2012; Blackman et al. 2012; Skrtic et al. 2011; Minear et al. 2011; Nislow \& Giaever, 2007). The cellular targets of FC-592 and FC-888 were not definitively identified by this technique in the current project however. In assessing the performance of this assay in this case there are several factors to consider.

The purpose of the assay is to identify hypersensitive deletion mutants which cluster around the target site. There are several issues that have been identified in relation to the microarray method and the assay type in particular in general that may have contributed towards the ambiguous results seen in this experiment. Firstly, the likelihood is that neither FC-592 nor FC-888 is large or structurally complex enough to provide a specific binding site in a cellular context.

This means that their mode-of-action is likely to be similarly non-specific, attacking targets and interfering with cellular processes in a very wide ranging manner. This non-specific mechanism therefore targets general cell fitness more than any individual process, function, or component, meaning hits within the dataset are not statistically enriched for any particular GO term. Many of the hits seen in the HOP screens conducted, especially for FC-888, mediate resistance to a wide range of small molecule inhibitors with diverse mechanisms. This is consistent with the theory that these genes mediate general fitness: the weakened cells are susceptible to attack against a broad range of targets.

If this is the case, then the yeast tag microarray HOP assay is doing exactly what it was designed to do, even if the answers are discouraging. However, it is difficult to prove a negative (that FC-592 and 
FC-888 do not target a specific process) in this way, especially considering the imprecise nature of the technique. Microarray data sets are commonly very large, and analytical precision is influenced by a number of variables (Groen, 2001), of both an experimental and analytical nature.

Arrays may contain errors because of cross contamination in the clones used for the spotting procedure (Knight, 2001). This is obviously a problem because the algorithm for reading the chip is based on the assumption that each oligonucleotide-spot is correctly labelled and hybridises in exactly the same ways as others of its kind. Standard deviation in z-scores amongst identical oligonucleotide-spots was found to be 0.19 . This result is not an unmanageable variance, however it still equates to a $7.53 \%$ average difference in percentile ranking between two spots which are in theory identical.

High sensitivity is an issue that is endemic to microarray experiments (Van Berkum \& Holstege, 2001). Tiny variations as a result experimental error are magnified because of the extremely sensitive nature of the signal produced in microarray experiments and of the instruments used in measuring them. This issue is magnified at very low signal levels where the low signal to noise ratios can make it difficult to assess whether or not a certain result is valid (Newton et al. 2001).

Microarrays are vulnerable to statistical variation caused by maximum and minimum signal strength (Smith et al. 2010). This is a result of hybridisation saturation, whereby oligonucleotide-spots become maximally saturated; and the threshold applied to low signal intensities, whereby oligonucleotide-spots displaying very low signal intensities are discarded. These artefacts are an unavoidable fact of life for most microarray methodologies, but may risk discarding good data in an effort to reduce noise.

This is not to say that microarrays are a flawed technology. As previously mentioned they have a long track record of success. Several methods exist for analysing data in an effort to resolve several of the above issues (Little \& Jones, 2011), which include various normalisation algorithms, increasing 
replicates, new methods for correcting basal hybridisation levels (Lee et al. 2000), and removal of dubious and poor quality signals from the data set (Colantuoni et al. 2002). The fact remains however that microarray experiments are inherently 'noisy', with a great deal of unexplained variation.

In a yeast tag microarray context, this means that it is impossible to 'guarantee' the appearance of a particular hit in a given dataset, even when that hit theoretically should appear. For this reason, it has proven impossible to detect linkage disequilibrium patterns in microarray datasets (Quek, 2011), even when those patterns of growth have been confirmed in a macro-scale, agar array based assay.

This is of course the reason that these datasets are analysed for statistical enrichment of certain GO terms, the theory being that any single gene hit is unimportant for identifying a target process, function or component: the totality of hits will point in the correct direction. With non-specific mechanisms however, such as have been supposed for FC-592 and FC-888, this statistical method breaks down. There is not enough enrichment in any one GO term to indicate a mechanism. Therefore other assays may have been more appropriate for the identification of a mode-of-action for these compounds. These could have included computational based methods that predict a target type based on structure and chemistry (McInnes \& Sykes, 1997). Basically this is a more detailed approach to the chemistry analysis conducted in predicting the activities of FC-592 and FC-888 in Chapter 2. In addition, suspected pathways could be assayed using biochemical methods such as the secretory pathway Gaussia luciferase assay (Badr et al. 2007).

A further issue with the yeast tag microarray has been identified with the oligonucleotide tags themselves (Smith et al. 2009). These researchers found that $\sim 20 \%$ of the barcodes and common priming sequences varied from expectation and thus were unable to achieve hybridisation in the required manner. This obviously has serious implications for the accuracy and relevance of the microarray. Although Smith et al. found that all 20 bp weren't necessary to achieve hybridisation, and thus it is not as simple as saying $\sim 20 \%$ of oligonucleotide-spots do not work, this result calls into 
question the efficacy of the yeast barcode microarray when using uncorrected data (Smith et al. produced corrected sequences and data based on their research; however this was not available to use in the current microarray experiment).

An alternative method that has been suggested takes advantage of next-generation sequencing technology to identify barcodes, known as Barcode analysis by Sequencing or Bar-Seq (Smith et al. 2009). This method was found to be superior to microarray based methods in relation to sensitivity, dynamic range and detection limits. In addition, bioinformatic resources can be used to apply these techniques to any genome which has been sequenced, obviating the need to design and produce microarray chips, an expensive process.

These advances were associated with reducing noise in relation to a previously identified drug target (Smith et al. 2009; Hoon et al. 2008). Therefore, such an approach may be useful in increasing resolution of the dataset and improving the chances of identifying relevant pathways and processes in experiments involving broad acting bioactive compounds.

\subsubsection{Unfolded protein response experiment}

Due to the range of hits indicating processes linked with glycoprotein biosynthesis and the sectretory pathway in the FC-592 HOP screen, an assay was conducted in an attempt to confirm activity of this compound on these processes. The thinking behind this is that disruption of certain enzymatic processes involved in these pathways, as indicated by certain hits from the assay, could lead to an accumulation of mis-folded proteins in the ER, triggering a series of cellular responses known as the Unfolded Protein Response (UPR). The UPR is mediated by the transcription factor Hac1p, whose translocation to the nucleus activates a promoter driving green fluorescent protein (GFP) expression. GFP expression in the reporter strain therefore acts as a proxy indicator of the UPR (Jonikas et al. 2009; Bernales et al. 2006; Okamuraa et al. 2000). 
In this experiment however, FC-592 did not produce a strong response, with GFP expression only increasing slightly above basal levels. The primary causes of UPR induction in a cellular context are perturbation in calcium homeostasis or redox status, elevated secretory protein synthesis, expression of misfolded proteins through mutation, sugar/glucose deprivation, or altered glycosylation (Kaufman, 2002).

If FC-592 is considered as a non-specific hydrophobic pocket binder, it could disrupt enzymatic activity in glycosylation proteins such as Alg6p and Alg8p by congregating in the active binding sites of such enzymes, thus preventing their native activity. This could cause altered glycosylation sufficient to trigger the UFP as described above. However, experimental results have shown that this is not the case.

It is certainly possible that inhibition of certain enzymes in this fashion, even a broad range of enzymes, would not result in an accumulation of mis-folded proteins into the ER lumen. Whilst disruption of $N$-linked glycosylation has been shown to initiate the UPR (Li et al. 2011), this repression was at a high level. A lower level of glycosylation disruption from a compound with a more general mechanism may not produce enough mis-folded protein to accumulate to the ER, whilst still causing sufficient disruption (perhaps at a downstream target or targets) to cause arrest of cellular growth.

The secretory pathway is an important cellular process. At each step along the process there are critical factors that determine how and if the protein will be processed, including regulation of transport, selection of particular proteins, and correct protein modification (Lodish \& Berk, 2003). Whilst strong inhibition at any single step may trigger the UPR, it is possible that minor insult against a range of processes along the secretory pathway could be sufficient for inhibition of cellular growth whilst remaining below the threshold of mis-folded proteins that would initiate the response. 
This is of course highly speculative. The likelihood is that FC-592 acts in a relatively non-specific fashion where there is only minimal enrichment of action amongst any specific pathway. With its large range of proteins and enzymatic processes, glycoprotein biosynthesis and the secretory pathway may be slightly more vulnerable to the compound than other processes. Lacking direct specificity to pathway single target however, it is unclear how FC-592 could be used as a therapeutic or genetic probe, although there is possible utility as a more general secretory pathway stressor. 


\section{Synthetic Genetic Array Mapping}

\subsection{Introduction}

\subsubsection{Resistance mutation as a target identification strategy}

The identification of the biological target of novel inhibitory compounds is a significant challenge (Ho et al. 2011). In recent times, identification of new drugs has become increasingly target based (Terstappen et al. 2007). However, these drug targets are often incompatible with conventional biochemical schemes of ligand identification (Chanda \& Caldwell, 2003), and this strategy has failed to accelerate drug discovery (Sams-Dodd, 2005). The new paradigm incorporates functional genomics approaches in a holistic strategy; often incorporating in vivo models (Terstappen et al. 2007; Lindsay, 2003) as well as genomic tools such as expression microarrays (Schena et al. 1998).

One in vivo strategy that has stood the test of time is resistance analysis (Justice et al. 1998; Fried \& Warner 1981; Fried \& Warner 1982; Liu et al. 1994). This involves the generation and characterisation of mutants resistant to the compound being studied, and has been used to identify the targets of many small molecule inhibitors over the years. These include nystatin (Ahmed and Woods, 1967) and reveromycin (Miyamoto et al. 2002) in S. cerevisiae, as well as neumocandin and echinocandin anti-biotics in C. albicans (Douglas et al. 1997).

The tractability and robustness of the genome in yeasts such as S. cerevisiae and C. albicans makes them uniquely suited for this type of experiment (Forsburg, 2001). The utility of this technique is served by the ease of manipulation of yeast strains, generating mutant populations easily and cost effectively in both a random (e.g. EMS) and site directed (e.g. PCR mediated gene disruption) manner. The nature of yeast cells mean that large numbers can be assayed for resistance on selective media in a way that is not possible with other model organisms. In addition, the haploid/diploid lifecycle of the likes of $S$. cerevisiae means that mutations can be recovered easily and complementation tests can be readily performed. 
Whilst resistant mutant generation is relatively easy, characterisation of the mutations responsible and linking those mutations to a precise mechanism of action remains a challenge. In the future, 'next generation DNA sequencing' (Shendure and Handlee, 2008), combined with bioinformatic analysis offers perhaps the most promising route for the rapid and efficient characterisation of SNP mutations. However, for some researchers high sequencing costs and low throughputs have limited the depth of analysis of genomic elements (Fullwood et al. 2009), especially relative to requirements for a high throughput strategy often required in drug discovery.

Several alternative approaches can be taken with S. cerevisiae that take advantage of the superlative range of genomics based tools available to the yeast researcher. Cloning by complementation, introducing a wild type copy of the gene to complement a drug hypersensitive phenotype is a commonly employed strategy (Douglas et al. 1994). In this strategy, a vector such as a plasmid library containing DNA fragments encompassing the entire genome rescues the wild type phenotype of transformed mutants, with each of the resulting transformants screened for drug sensitivity. This technique, although widely used (Douglas et al. 1994; Parsons et al. 2006; Justice et al. 1998; Heitman et al. 1991), requires large amounts of effort to assay a vast number of transformants.

In more modern times a complementation strategy has been used that takes advantage of microarray barcode technology to identify recessive resistant mutations (Ho et al. 2009). Molecular barcoded yeast open reading frames (MoBY-ORFs), each barcode unique to a genomic location, are constructed as a plasmid library. This allows population analysis of transformants based on barcode expression in a manner analogous to chemigenomic microarray profiling described in the previous chapter. Cells containing a recessive resistance mutation are transformed and grown in the presence of the compound of interest. The rescued phenotype (those cells containing the complementary barcode tagged wild type ORFs) does not grow in the presence of the drug, whilst all others do. This absence is determined on a barcode microarray, revealing the site of resistance. This advance is particularly important when limited quantities of the compound of interest are available. 
Similar approaches utilising more classical techniques have been utilised in the past. Fried and Warner (1981) identified RPL3, a protein component of the large (60S) ribosomal subunit, as the molecular basis of resistance for a range of eukaryotic protein synthesis inhibitors such as trichodermin and verrucarin $\mathrm{A}$ as well as to the unrelated drug anisomycin. This was achieved through transforming trichodermin sensitive HF-T1 mutants with a cloned recombinant plasmid library carrying wild type genomic DNA fragments. These transformed mutants were grown on trichodermin resistance selective media and the surviving cells (containing the relevant trichodrmin resistance allele) sequenced using a plasmid based primer. In this way, sequencing efficiency is achieved because the entire genome does not have to be sequenced: only the relevant allele contained within the plasmid.

These techniques allow an assessment of the mechanism of resistance in vivo, as opposed to traditional biochemical methods which directly focus on target affinity (Lindsay, 2003). This is important because resistance to a compound can be caused in a variety of ways, not limited to the direct binding of the target. For example, a loss-of-function mutation in an enzyme required for activation of some part of a compound's toxicity could lead to a resistant phenotype, and reveals much about the mechanism of the compound in question (Heitman et al. 1991). This whole cell approach is a key advantage of resistance based target analysis.

\subsubsection{Synthetic genetic array mapping}

As mentioned previously, researchers have constructed a library of deletion mutants in S. cerevisiae that consists of all non essential genes disrupted by a kanamycin resistant marker (Winzeler et al. 1999), known as the deletion mutant array (DMA). This genomic reagent's utility has been discussed in regards to chemigenomic analysis, examining strains within the DMA that are hypersensitive to a compound of interest and the implications of such hypersensitivity for indentifying that compound's mechanism of action. 
The DMA possesses another utility as well. By mass mating the DMA against a query strain

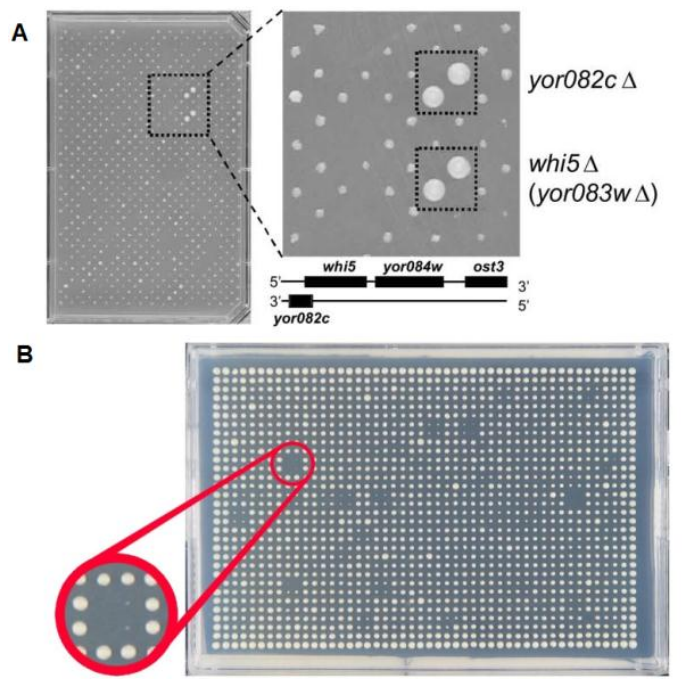

Figure 4.1 Examples of SGA methodology. (A) Isolation of whi5 in a systematic genetic screen for inhibitors of SCB dependent transcription. A cln3 strain containing an SCBHIS3 reporter (BY2054) was mated to 4812 haploid strains. This identifies deletion strains that rescue a phenotype. Source: Costanzo et al. 2004. (B) Enhanced synthetic lethal (SL) phenotype shows genetic interaction in an SGA Source: Victoria University of Wellington Chemical Genetics Laboratory. containing a single gene knockout, it is possible to readily create libraries of double mutants that contain two gene knockouts with selectable markers (Tong et al. 2001). When such double mutations appear in differing pathways, they are generally buffered against deleterious effect by redundant genes and pathways within their networks. However, when they are closely related, for example acting within parallel pathways, the cell is unable to overcome this dual insult to an essential process and an enhanced phenotype is seen. This typically takes the form of a synthetic sick

(SS) or synthetic lethal (SL) phenotype that displays reduced or null vegetative growth. Such phenotypic enhancements indicate genetic interactions between gene pairs, and in this way it is possible to build a picture of large scale genetic networks of overlapping pairs of SL interactions. This

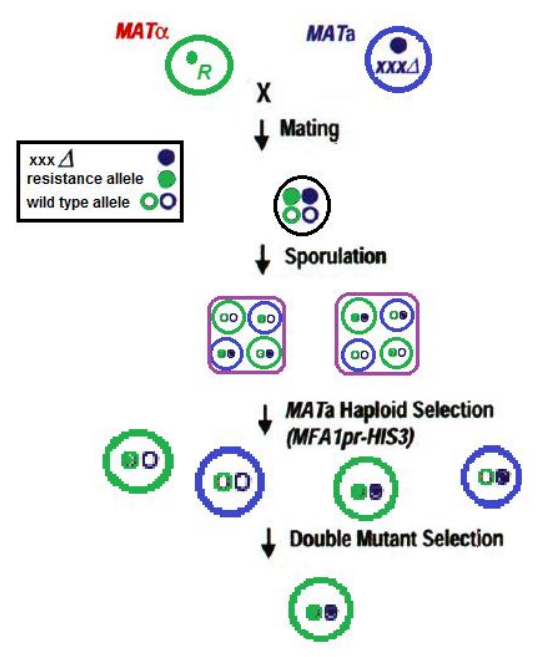

Figure 4.2 Synthetic genetic array mapping. Only strains carrying both the resistance allele and a KanR cassette from the DMA are able to grow under double selection. Adapted with permission from AAAS: Science, Tong et al. 2001. methodology is known as the Synthetic Genetic Array, or SGA.

SGA methodology has been used frequently and successfully for a range genetic research: from leveraging synthetic lethality as a means for selective killing of tumour cells (McClellan et al. 2012), to exploring the heredity of gene networks (Yang \& Sankoff 2011), as well as matching gene function to gene and vice versa (Short et al. 2012; Ball et al. 2009). The genome-wide selection markers present within the DMA allow an elegant alternative use, identifying the 
genomic location of a selectable trait, known as Synthetic Genetic Array Mapping or SGAM (Jorgensen et al. 2002).

When the selectable trait is a resistance mutation, SGA methodology can be used to trace the source of resistance. The resistant strain is mass mated against the DMA and grown on selective media such that only progeny strains containing both the resistant allele and the kanamycin (G418) resistance cassette

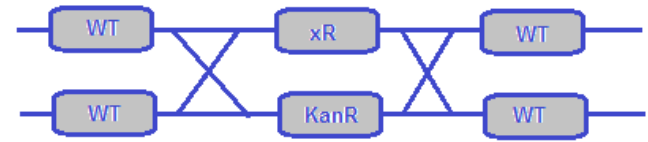
will grow in the presence of both drugs. In an SGAM strategy, this only occurs when the drug resistance gene and the $\mathrm{G} 418$ resistance gene are not the same or

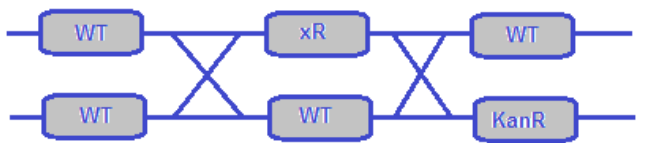

Figure 4.3 Homologous recombination in SGAM. Where the KanR cassette and the unknown resistance mutation $(\mathrm{xR})$ are in separate regions of the genome, cross over located in closely linked loci. When the G418 resistance events allow double mutants which survive under double selection (bottom). When the resistance loci are closely linked or identical, these cross over events are rare or nonallele and the drug resistance alleles are on the same or a nearby locus then strains will not grow in presence existant (if allelic) and the double mutants do not occur, causing cells to die from sensitivity to one or other of the drugs.

of both drugs, since haploid alleles cannot occupy the same locus. Owing to linkage disequilibrium,

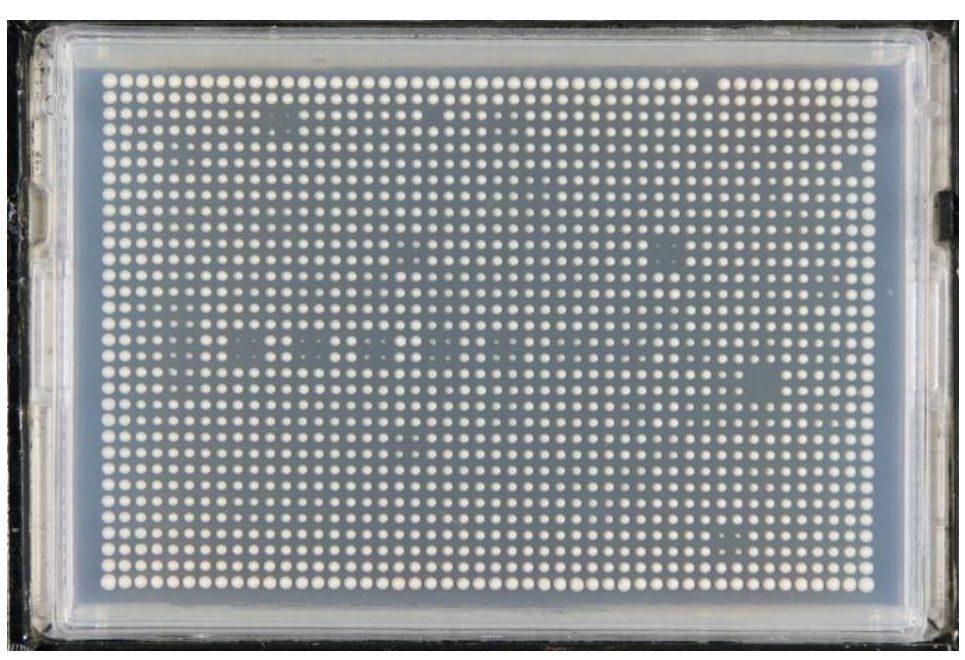

Figure 4.4 A linkage pattern is seen around the site of resistance in a SGAM experiment. Growth defect is maximal around the center of this region, where the fewest cross over events occur during meiosis relative to the KanR cassette. Source: Ploi Yibmantasiri. strains with the G418 resistance allele immediately neighbouring the drug resistance allele will also be unable to grow under the double selective conditions, leaving a distinctive linkage pattern of nongrowth within the final array. The centre of this pattern serves to identify the region of the genome in

which the resistance mutation is located (Yibmantasiri et al. 2012).

Because the DMA is arranged in a specific genomic order, the linkage pattern is correlated to identifiable genes. These may not directly correspond to the resistance mutation, which may be 
located on an essential gene which does not occur in the DMA. However, the linkage pattern nonetheless forms from neighbouring genes because of reduced homologous recombination relative to the KanR cassette. Therefore genes represented by strains within the linkage pattern implicate neighbouring gene deletions bearing the replacement G418-reistance cassette as probable sites of the resistance mutation.

It should be noted that a key weakness of the SGAM technique is the quantity of drug required to treat the $\sim 14$ plates typically present in an SGA assay, a relatively large total volume of growth medium. This is limiting and may prove prohibitive for novel compounds which are frequently in short supply. It has been suggested that conducting such experiments in barcode-tagged microarray format, with a pooled DMA mass mated against a resistant strain, may be effective in reducing the amount of drug required. However the resolution achieved in microarrays has proven insufficient to identify linkage within a pooled array in this manner (Quek, 2011). Simply put, there is too much noise implicated in microarray experiments to make them viable in a SGAM strategy.

\subsubsection{Flow cytometry}

Flow cytometry is a laser based, biophysical technique used to count and sort cells based upon a measurable biomarker. This high-throughput technique is achieved by suspending cells in a stream of fluid and passing them by an electronic detection device. In this way, multiparametric analysis is possible on a scale that allows statistical interpretation of biological phenomena (Loken, 1990). One common utility of flow cytometry is for the analysis of cell proliferation in the study of the cell cycle (Gray et al. 1986).

In this application, cells are fixed at a certain point during or after treatment with the studied condition. Cells are stained with a DNA binding dye such as SYTOX Green (Haase \& Reed, 2002), which enables DNA content to be quantified via fluorescence activated cell sorter (FACS), determining cell cycle progression of each cell. Effects of the studied condition (which might be a 
particular mutation or a small molecule drug) on the cell cycle can be determined by examining the proportion of cells in $G_{1}, G_{2}$ etc. (Sohn et al. 2000).

\subsection{Aims and objectives}

The aim of this chapter is to provide evidence for the identification of the mechanism of action of FC-592 by performing a Synthetic Genetic Array Mapping experiment. The key objectives are:

1. Generate several S. cerevisiae mutants resistant to FC-592.

2. Perform a proof of concept SGAM experiment using the cycloheximide resistant strain YCG191.

3. Perform complementation analysis of resistant mutants to establish nature of mutations.

4. Run SGAM procedure using at least one resistant mutant

5. Identify site of resistance by determining linkage pattern in SGAM results

\subsection{Methods and materials}

\subsubsection{Yeast strains}

\begin{tabular}{|c|c|}
\hline Strain & Genotype \\
\hline BY4742 & 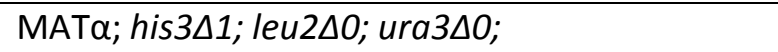 \\
\hline BY4741 & 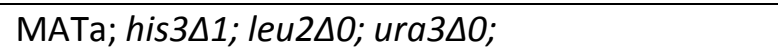 \\
\hline Y7092 & 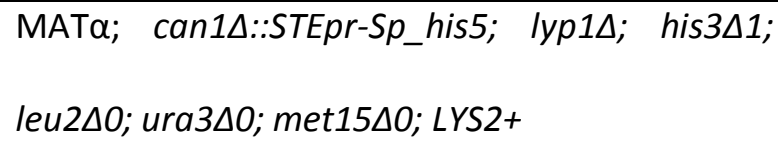 \\
\hline YCG326 & 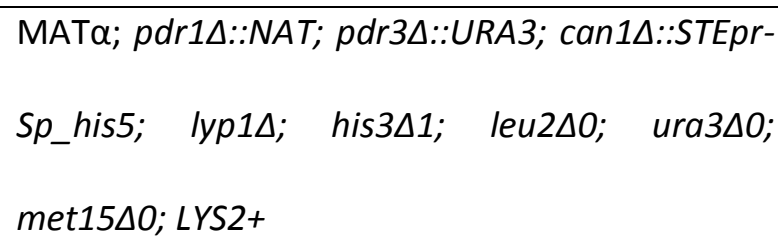 \\
\hline
\end{tabular}




\begin{tabular}{|c|c|}
\hline $\operatorname{Pdr} \Delta ; \mathrm{His} 3 \Delta$ & 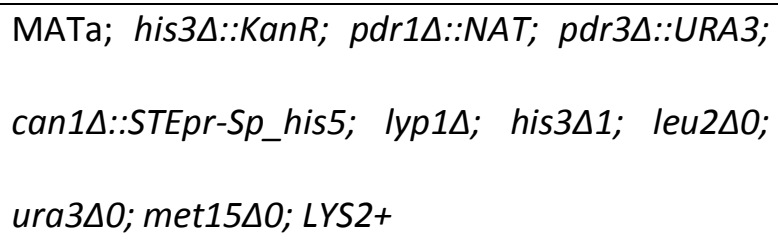 \\
\hline YCG191 & 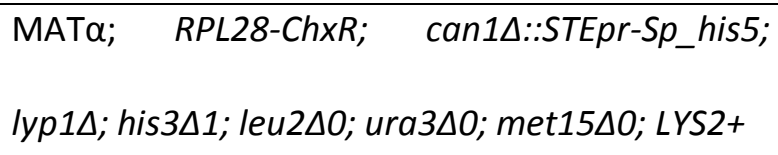 \\
\hline YCG432 & 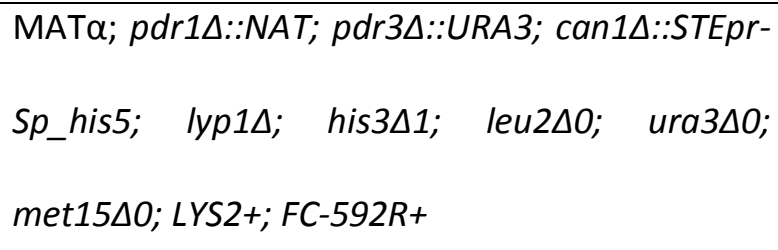 \\
\hline YCG433 & 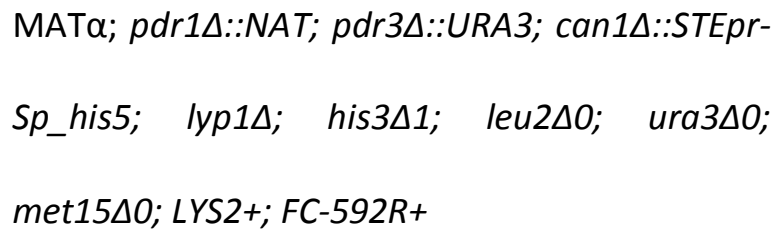 \\
\hline YCG434 & 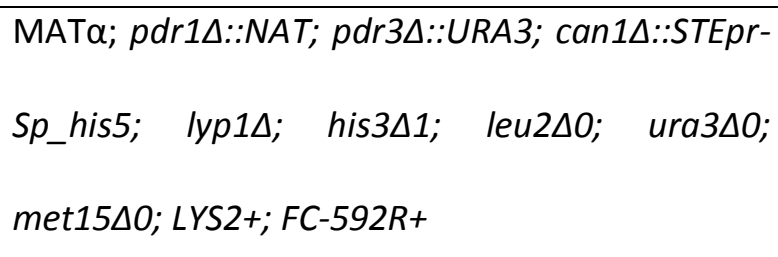 \\
\hline YCG435 & 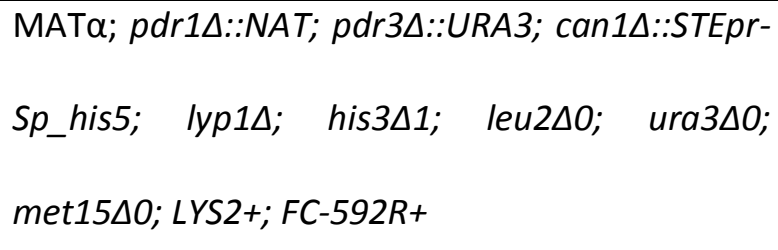 \\
\hline YCG436 & 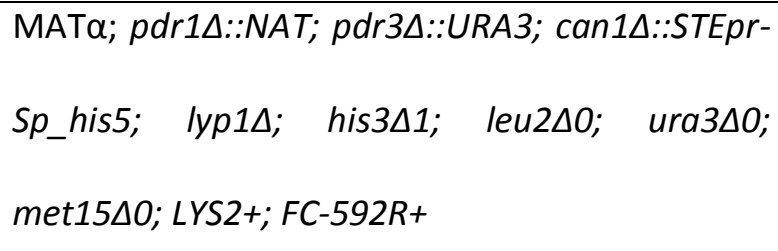 \\
\hline $\begin{array}{l}\text { Boone Deletion Mutant Array (DMA; Winzeler et } \\
\text { al. 1999) }\end{array}$ & 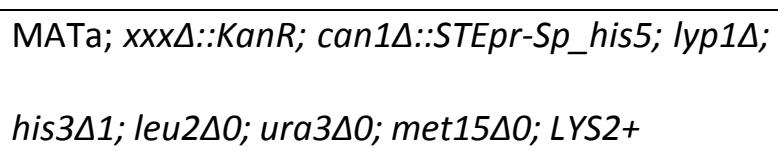 \\
\hline
\end{tabular}




\subsubsection{Growth media}

Yeast growth assays were performed in the following media formulations. All media was made up with distilled deionised $\mathrm{H}_{2} \mathrm{O}\left(\mathrm{ddH}_{2} \mathrm{O}\right)$ and autoclaved at $120^{\circ} \mathrm{C}$ for $20 \mathrm{~min}$. Sterile glucose was added to media post autoclave:

Yeast peptone dextrose (YPD): As in Chapter 2.

Synthetic complete (SC): As in Chapter 2.

Synthetic deficient (SD) - Ura: As in Chapter 2.

Synthetic deficient (SD) - His: As SC except without histidine in the amino acid mix

Synthetic deficient (SD) - Arg: As SC except without arginine in the amino acid mix

Synthetic deficient (SD) - Lys: As SC except without lysine in the amino acid mix

Synthetic deficient (SD) - Leu: As SC except without leucine in the amino acid mix

GNA: 3\% (w/v) nutrient broth (Acumedia), 1\% yeast extract (Bacto), 2\% select agar (Invitrogen), 5\% glucose, $0.012 \%$ adenine hemisulfate.

Sporulation (SPO): $1 \%$ potassium acetate, $0.005 \%$ zinc acetate, $0.1 \%$ yeast extract (Bacto), $0.01 \%$ sporulation supplement (2 $g$ histidine, $2 \mathrm{~g}$ uracil, $10 \mathrm{~g}$ leucine; $2 \mathrm{~g}$ lysine; all SigmaAldrich), 0.05\% Dglucose.

Agar: As in Chapter 2.

NAT: As in Chapter 2.

G418: As in Chapter 2. 
Can: Where noted, $50 \mathrm{mg} / \mathrm{mL}$ (final concentration) canavanine (SigmaAldrich) was added to media after sterilization.

Thia: Where noted, $50 \mathrm{mg} / \mathrm{mL}$ (final concentration) thialysine (SigmaAldrich) was added to media after sterilization.

\subsubsection{Compounds and chemicals}

Ethyl methanesulfonate (EMS, SigmaAldrich), sodium thiosulfate, sodium phosphate $(19.5 \% 200 \mathrm{mM}$ $\mathrm{NaH}_{2} \mathrm{PO}_{4}, 30.5 \% 200 \mathrm{mM} \mathrm{NaHPO}_{4}, 50 \% \mathrm{ddH}_{2} \mathrm{O}$ ) and sodium hydroxide were used in a mutagenesis experiment to introduce genetic variation in yeast populations.

A Geneaid High-Speed Plasmid MiniPrep kit was used to purify plasmids for transformation. Lithium acetate (SigmaAldrich), polyethylene glycol (SigmaAldrich), salmon sperm single stranded DNA (SigmaAldrich), Tris-EDTA (Fischer Scientific), sodium hydroxide and absolute ethanol were used in plasmid transformations. Zymolase (Invitrogen) and sorbitol (SigmaAldrich) were used during tetrad analysis. Pepsin (SigmaAldrich), RNase A (Qiagen), absolute ethanol, Tris-HCl (SigmaAldrich) and SYTOX Green (Invitrogen) were used during cell fixing and staining for the cell cycle flow cytometry experiment.

Cycloheximide (SigmaAldrich), rapamycin (LC Laboratories), hygromycin B (SigmaAldrich), fluconazole (SigmaAldrich) and nystatin (SigmaAldrich) were dissolved in DMSO at a range of concentrations for comparative analysis. Benomyl (SigmaAldrich) was dissolved in $\mathrm{ddH} 2 \mathrm{O}$ for the same purpose. All compounds were stored at $-20^{\circ} \mathrm{C}$.

\subsubsection{Resistant mutant generation}

Ethyl methanesulfonate (EMS) mutagenesis was performed on a parent population of YCG326 pdrA cells. Safety note: EMS is a powerful mutagen. Perform all EMS work in a fume hood. Discard any EMS containing liquids into a beaker containing $50 \%$ sodium thiosulfate solution. Wash all EMS containing material with $10 \%$ sodium thiosulfate solution. All glassware coming into contact with 
EMS must be immersed in a large beaker of $1 \mathrm{M} \mathrm{NaOH}$ before washing and reuse. Ensure EMS never touches the outside of tubes, gloves etc.

A single colony of YCG326 was grown overnight in $3 \mathrm{~mL} \mathrm{SC}$ at $30^{\circ} \mathrm{C}$ on a rotating drum. Cell titre was determined via haemocytometer and diluted to $2 \times 10^{8}$ cells $\mathrm{mL}^{-1}$ with fresh SC. $1 \mathrm{~mL}$ samples were transferred to sterile $1.5 \mathrm{~mL}$ microcentrifuge tubes, centrifuged for $30 \mathrm{~s}$ at $13000 \mathrm{rpm}$, supernatant discarded and cells resuspended in $1 \mathrm{~mL} d \mathrm{ddH}_{2} \mathrm{O}$. This wash step was repeated, with supernatant being discarded leaving a cell pellet which was resuspended in $1 \mathrm{~mL}$ of $100 \mathrm{mM}$ sodium phosphate. $30 \mu \mathrm{L}$ EMS was added to one tube for a final concentration of 3\% (with the other tube serving as the unmutagenised control). Tubes were vortexed vigorously to mix and incubated at $30^{\circ} \mathrm{C}$ for $1 \mathrm{~h}$ on a rotating drum. The tubes were then centrifuged at $13000 \mathrm{rpm}$ for $30 \mathrm{~s}$, supernatant discarded (into breaker containing $50 \%$ sodium thiosulfate), and cells resuspended in $20 \mu \mathrm{L} 5 \%$ sodium thiosulfate. These suspensions were transferred into fresh sterile $15 \mathrm{~mL}$ microcentrifuge tubes and centrifuged at $13000 \mathrm{rpm}$ for $30 \mathrm{~s}$, supernatant discarded and resuspended in $5 \%$ sodium thiosulfate. This wash step was repeated once more, before supernatant was discarded and cells resuspended in $1 \mathrm{~mL}$ $\mathrm{ddH}_{2} \mathrm{O}$. These suspensions were transferred into $15 \mathrm{~mL}$ falcon tubes, centrifuged for $2 \mathrm{~min}$ at 4000 rpm, supernatant discarded and cells resuspended in $3 \mathrm{~mL}$ YPD.

After mutagenesis, a viability assay was conducted to ensure adequate mutation level. Cell titre of mutagenised and unmutagenised populations were determined via haemocytometer and diluted to $5 \times 10^{5}$ cells $\mathrm{mL}^{-1}$ in $\mathrm{ddH}_{2} \mathrm{O} .20 \mu \mathrm{L}$ aliquots of these cell suspensions were pipetted into $200 \mu \mathrm{L}$ pools of ddH2O which had been dispensed onto the surface of SC Agar plates to aid spread. These pools were spread over the surface with the aid of a sterile glass rod, and grown for $\sim 48 \mathrm{~h}$ at $30^{\circ} \mathrm{C}$. Plates were visually examined to ensure desired attrition rate had been achieved (10-30\%).

Simultaneously, the cultures were grown at $30^{\circ} \mathrm{C}$ for $\sim 4 \mathrm{~h}$ on a rotating drum to allow mutant proteins to replace wild type proteins in the population. 


\subsubsection{Screening for $\mathrm{FC}$-592 resistance}

The mutant populations generated in the EMS mutagenesis experiment were screened for resistance to FC-592. $50 \mu \mathrm{L}$ samples of mutagenised and unmutagenised cells were harvested after outgrowth and transferred into $1.5 \mathrm{~mL}$ microcentrifuge tubes. Cells were washed with $1 \mathrm{~mL} d \mathrm{dH}_{2} \mathrm{O}$, centrifuged at $13000 \mathrm{rpm}$ for $30 \mathrm{~s}$ and supernatant discarded, and resuspended in $1 \mathrm{~mL} \mathrm{dd} \mathrm{H}_{2} \mathrm{O}$. Cell titre of these suspensions was determined via haemocytometer and diluted to $1 \times 10^{6}$ cells mL ${ }^{-1}$ with $\mathrm{ddH}_{2} \mathrm{O} .200 \mu \mathrm{L}$ aliquots (for a total of $\sim 200,000$ cells) of mutagenised and unmutagenised cells were spread on five (each) SC Agar plates containing FC-592 (50 $\mu \mathrm{M}, 70 \mu \mathrm{M}, 90 \mu \mathrm{M}$ and $110 \mu \mathrm{M})$. These plates were grown at $30^{\circ} \mathrm{C}$ for $\sim 48 \mathrm{~h}$. Plates were visually inspected for growing colonies, with resistant mutants selected from those plates containing an FC-592 that completely inhibited growth on the unmutagenised plates.

Resistant characteristics of these mutants (designated YCG432, YCG433, YCG434, YCG435 and YCG436) were determined via solid state dose response. A half-log dilution series was created in DMSO for FC-592 with final concentrations of $354.6 \mu \mathrm{M}, 112.1 \mu \mathrm{M}, 35.5 \mu \mathrm{M}, 11.2 \mu \mathrm{M}, 3.55 \mu \mathrm{M}, 1.12$ $\mu \mathrm{M} \& 355 \mathrm{nM}$. A solid state dose response plate was created in a clear flat-bottom 48-well plate (Interlab), according to the protocol set out in Chapter 2. Single colony cultures of each resistant mutant, as well as a YCG326 control, were grown overnight in YPD, cell titre determined via haemocytometer and diluted to $5 \times 10^{5}$ with fresh YPD and cell suspension vortexed to ensure homogenization. $2 \times 2 \mu \mathrm{L}$ of each suspension was spotted into separate wells (such that each strain would be challenged against each concentration), allowed to diffuse for 5 min then incubated for $\sim 40 \mathrm{~h}$ at $30^{\circ} \mathrm{C}$. Photographs (Canon Powershot S3-IS) were then taken using ZoomBrowser EX remote shooting software (Canon Inc, NY, USA) and visually inspected to determine cell growth.

\subsubsection{Tetrad analysis}

Resistant mutants were characterised by tetrad analysis. Single colonies of YCG432, YCG433, and YCG434 were transformed with cenLeu plasmid extracted from the pRS315 E. coli strain (Geneaid 
High-Speed Plasmid MiniKit) according to the protocol outlined in Geitz and Schiestl (2008). Transformants were confirmed by growth on SD-Leu Agar, and single colonies mated to $\operatorname{Pdr} \Delta: \mathrm{His} 3 \Delta$ according to the protocol outlined in Burke et al. (2005). Mated cells were replica plated on to SDLeu G418 Agar diploid selection media and grown at $30^{\circ} \mathrm{C}$ for $\sim 24 \mathrm{~h}$. Diploid selection plates were then replica plated onto GNA pre-sporulation media and grown at $30^{\circ} \mathrm{C}$ for $\sim 12 \mathrm{~h}$. $\sim 500 \mathrm{mg}$ of diploid cells from GNA plates were transferred via sterile rod into $3 \mathrm{~mL}$ SPO media, briefly vortexed, and incubated for $\sim 5$ days at $24^{\circ} \mathrm{C}$ on a rotating drum. Cells were visually inspected via microscopy to ensure adequate spore formation.

$150 \mu \mathrm{L}$ aliquots of each sporultated strain were dispensed into sterile $1.5 \mathrm{~mL}$ microcentrifuge tubes, centrifuged at $13000 \mathrm{rpm}$ for $30 \mathrm{~s}$ and supernatant discarded. $50 \mu \mathrm{L} 0.25 \mathrm{mg} \mathrm{ml}^{-1}$ zymolase in $1 \mathrm{M}$ sorbitol was added to each tube and incubated at $30^{\circ} \mathrm{C}$ for $30 \mathrm{~min}$ before being quenched with 150 $\mu \mathrm{L} \mathrm{ddH}_{2} \mathrm{O}$ and cooled on ice. Tetrads were then spread on thin YPD Agar plates and dissected according to the protocol outlined in Burke et al. (2005) using a dissection microscope (Singer Instruments MSM System).

Dissected tetrads were allowed to grow at $30^{\circ} \mathrm{C}$ for $\sim 48 \mathrm{~h}$ before being replica plated onto the following media types: SD-Leu Agar, SC G418 Agar. Cells from selected tetrads were manually transferred onto SC FC-592 (150 $\mu \mathrm{M})$ Agar media by picking colonies with a sterile rod, transferring into $1.5 \mathrm{~mL}$ microcentrifuge tube containing $1 \mathrm{~mL}$ sterile $\mathrm{dd}_{\mathrm{H}} \mathrm{O}$, vortexing to mix, and dispensing 1 $\mu \mathrm{L}$ aliquots. This was done because SC FC-592 (150 $\mu \mathrm{M})$ Agar media was made up in smaller $8 \mathrm{~mL}$ Petri plates, in an effort to minimise the amount of compound used.

\subsubsection{Dominance/recessive testing}

Separately, diploid cells resulting from mating during tetrad analysis were assayed for resistance to FC-592 to establish whether each mutant displayed dominant or recessive resistance. A single colony of YCG432×Pdr $\Delta ; H$ His3 $\Delta, Y C G 433 \times P d r \Delta ; H i s 3 \Delta$ and $Y C G 434 \times P d r \Delta ; H i s 3 \Delta$ diploids were transferred via sterile rod into $1.5 \mathrm{~mL}$ microcentrifuge tubes containing $1 \mathrm{~mL} \mathrm{ddH_{2 }} 0$ and vortexed. A $10 x$ serial 
dilution series was constructed from these cells to produce a range of cell suspensions in descending cellular concentrations. $1 \mu \mathrm{L}$ aliquots of these cell suspensions were dispensed onto SC FC-592 (150 $\mu \mathrm{M}$ ) Agar, along with YCG 326 \& YCG434 controls, and grown for $\sim 48 \mathrm{~h}$ at $30^{\circ} \mathrm{C}$. Photographs (Canon Powershot S3-IS) were then taken using ZoomBrowser EX remote shooting software (Canon Inc, NY, USA) and visually inspected to determine cell growth.

\subsubsection{Complementation testing}

A complementation test was conducted for YCG433 and YCG434 to determine whether the two recessive mutations were in the same functional unit or gene. Two recessive mutant strains crossed together complement each other if the resulting diploid has a wild type phenotype. A Mat A KanR+ cenLeu- YCG434 mutant progeny was selected from tetrad analysis and crossed according to the protocol in Burke et al. (2005) with a YCG433 cenLeu+ transformant. Diploid selection was carried out on SD-Leu G418 Agar media and a single colony transferred via sterile rod into $1.5 \mathrm{~mL}$ microcentrifuge tubes containing $1 \mathrm{~mL} \mathrm{ddH_{2 }} \mathrm{O}$ and vortexed. $1 \mu \mathrm{L}$ aliquots of this suspension, along with YCG326 and YCG434 controls, were dispensed onto a SC FC-592 Agar dose response (150 $\mu \mathrm{M}$, $135 \mu \mathrm{M}, 120 \mu \mathrm{M}, 105 \mu \mathrm{M}, 90 \mu \mathrm{M}, 75 \mu \mathrm{M}$ and 1\% DMSO) and grown for $\sim 48 \mathrm{~h}$ at $30^{\circ} \mathrm{C}$. Photographs (Canon Powershot S3-IS) were then taken using ZoomBrowser EX remote shooting software (Canon Inc, NY, USA) and visually inspected to determine cell growth.

\subsubsection{Multidrug resistance analysis}

In order to establish the specificity of resistance mutations in YCG433 and YCG434 to FC-592, dose responses were carried out in liquid media against a range of other known inhibitory compounds. Dilutions (all final concentrations) of rapamycin (1000 nM, $316 \mathrm{nM}, 100 \mathrm{nM}, 31.6 \mathrm{nM}, 10 \mathrm{nM}, 3.16$ $\mathrm{nM}, 1 \mathrm{nM})$; cycloheximide (1000 nM, $316 \mathrm{nM}, 100 \mathrm{nM}, 31.6 \mathrm{nM}, 10 \mathrm{nM}, 3.16 \mathrm{nM}, 1 \mathrm{nM})$; hygromycin B (317.1 $\mu \mathrm{M}, 117.4 \mu \mathrm{M}, 31.7 \mu \mathrm{M}, 11.7 \mu \mathrm{M}, 3.17 \mu \mathrm{M}, 1.17 \mu \mathrm{M}, 317.1 \mathrm{nM})$; benomyl (100 $\mu \mathrm{M}, 31.6$ $\mu \mathrm{M}, 10 \mu \mathrm{M}, 3.16 \mu \mathrm{M}, 1.0 \mu \mathrm{M}, 316 \mathrm{nM}, 100 \mathrm{nM})$; fluconazole $(32.6 \mu \mathrm{M}, 10.32 \mu \mathrm{M}, 3.26 \mu \mathrm{M}, 1.03 \mu \mathrm{M}$, $326 \mathrm{nM}, 103 \mathrm{nM}, 32.6 \mathrm{nM})$; nystatin $(10.75 \mu \mathrm{M}, 3.4 \mu \mathrm{M}, 1.075 \mu \mathrm{M}, 340 \mathrm{nM}, 107.5 \mathrm{nM}, 34 \mathrm{nM}, 10.75$ 
$n M)$ and FC-592 (150 $\mu \mathrm{M}, 47.43 \mu \mathrm{M}, 15 \mu \mathrm{M}, 4.74 \mu \mathrm{M}, 1.5 \mu \mathrm{M}, 474.3 \mathrm{nM}, 150 \mathrm{nM}, 47.4 \mathrm{nM})$ were prepared in a 96-well V-bottomed plate (Interlab).

Single colonies of YCG326, YCG433 and YCG434 were inoculated into $3 \mathrm{~mL}$ of SC broth and incubated at $30^{\circ} \mathrm{C}$ on a $40 \mathrm{rpm}$ rotary drum overnight. Cell titre was determined via haemocytometer, diluted with fresh SC to a final concentration of $5 \times 10^{5}$ cells $\mathrm{mL}^{-1} .100 \mu \mathrm{L}$ aliquots of these suspensions were dispensed (Eppendorf Xplorer Plus 8 Channel Electronic Pipette) into each well of a clear 96 well flatbottom polystyrene cell culture plate (Interlab) and $1 \mu \mathrm{L}$ aliquots of each dilution of test compound were dispensed in triplicate using a multi-channel pipettor (Eppendorf Research Plus 8 Channel) into these wells, along with $1 \%$ DMSO controls. Plates were grown and assayed for growth as per the dose response protocol (Chapter 2).

\subsubsection{Growth rate comparison of resistant mutants}

In order to ensure that YCG433 and YCG434 displayed an appropriate growth rate relative to wild type cells, a growth rate comparison assay was performed. Single colonies of YCG326, YCG433 and YCG434 were inoculated into $3 \mathrm{~mL}$ of SC broth and incubated at $30^{\circ} \mathrm{C}$ on a $40 \mathrm{rpm}$ rotary drum overnight. Cell titre was determined via haemocytometer, diluted with fresh SC to a final concentration of $5 \times 10^{5}$ cells $\mathrm{mL}^{-1} .100 \mu \mathrm{L}$ aliquots of these suspensions was dispensed (Eppendorf Xplorer Plus 8 Channel Electronic Pipette) into each well of a clear 96 well flat-bottom polystyrene cell culture plate. Cells were incubated at $30^{\circ} \mathrm{C}$ and assayed for growth as per the dose response protocol (Chapter 2) at $1 \mathrm{~h}$ timepoints from $12-24 \mathrm{~h}$.

\subsubsection{Synthetic genetic array mapping - proof of concept}

A pilot synthetic genetic array mapping (SGAM) experiment was carried out to confirm the utility of the technique. In order to minimise the amount of compound used up in this experiment, the DMA was condensed down to seven plates from 14, utilising duplicate rather than quadruplicate repeats of each strain. A synthetic array was constructed using the cycloheximide resistant strain YCG191 (which contains a mutation on the ribosomal protein encoding gene RPL28) and the Boone DMA 
according to the protocol set out in Baryshnikova et al. (2010), using a Singer Instruments RoToR HDA pinning robot. The final selection of haploid double mutants was pinned onto SD - His - Arg -Ura -Lys Can Thia G418 (10 $\mu \mathrm{M}$ cycloheximide) Agar media, and allowed to grow at $30^{\circ} \mathrm{C}$ for $\sim 18 \mathrm{~h}$. Photographs (Canon Powershot S3-IS) were recorded using ZoomBrowser EX remote shooting software (Canon Inc, NY, USA) and visually inspected to determine cell growth.

\subsubsection{Synthetic genetic array mapping - FC-592 resistant mutant}

The SGAM technique was utilised in an attempt to determine the site of the resistant allele in YCG434. A synthetic array was constructed using YCG434 and the Boone DMA as for the SGAM pilot experiment set out above. The final selection of haploid double mutants was pinned onto SD-His -

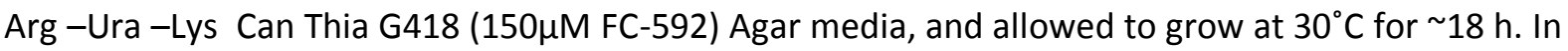
an attempt to ameliorate patchy growth observed in earlier selection stages, the final selection step onto FC-592 media was pinned twice, in order to seed more yeast cells onto the plate. Plate images (Canon Powershot S3-IS) were recorded using ZoomBrowser EX remote shooting software (Canon Inc, NY, USA) and visually inspected to determine cell growth.

\subsubsection{Cell cycle analysis by flow cytometetry}

Cell cycle effects of FC-592 were analysed by SYTOX visualisation of chromosome content by flow cytometry. A mid log-phase cell population was prepared by inoculating a single colony of YCG326 into $\sim 50 \mathrm{~mL} \mathrm{SC}$ in a conical flask and grown for $12 \mathrm{~h}$ at $30^{\circ} \mathrm{C}$ in a shaking incubator. Cell titre was determined via haemocytometer and diluted to $2 \times 10^{7}$ cell $\mathrm{mL}^{-1}$ with fresh SC. Two sets of triplicate 5 $\mathrm{mL}$ cultures were transferred to $15 \mathrm{~mL}$ Falcon tubes and treated with $100 \mu \mathrm{M} \mathrm{FC}-592$ or $1 \% \mathrm{DMSO}$. Cultures were grown at $30^{\circ} \mathrm{C}$ on a rotating drum for $6 \mathrm{~h}$.

After treatment, cultures were centrifuged at $4000 \mathrm{rpm}$ for $1 \mathrm{~min}$ and supernatant discarded. Cells were washed with $5 \mathrm{~mL} \mathrm{ddH} \mathrm{H}_{2} \mathrm{O}$, centrifuged at $4000 \mathrm{rpm}$ for $1 \mathrm{~min}$ and supernatant discarded. This wash step was repeated once more. Supernatant was discarded a final time and cells resuspended in $70 \%$ ethanol (from absolute) to fix. These cells were then incubated overnight at $4^{\circ} \mathrm{C}$. 
Tubes were centrifuged at $4000 \mathrm{rpm}$ for $1 \mathrm{~min}$ and EtOH supernatant discarded. Cells were transferred to sterile $1.5 \mathrm{~mL}$ microcentrifuge tubes and resuspended in $1 \times R$ Nase $A$ solution and incubated at $37^{\circ} \mathrm{C}$ for $1 \mathrm{~h}$. Cells were then washed with $\mathrm{ddH} 2 \mathrm{O}$, centrifuged, supernatant discarded and treated with $1000 \mu \mathrm{L}$ freshly prepared Pepsin solution $\left(0.005 \mathrm{~g} \mathrm{~mL}^{-1}\right.$ Pepsin, $\left.50 \mathrm{mM} \mathrm{HCl}\right)$ for 5 min at room temperature. Cells were centrifuged, supernatant discarded and the pellet resuspended in $500 \mu \mathrm{L} 50 \mathrm{mM}$ Tris- $\mathrm{HCl}$ (pH 7.5). $100 \mu \mathrm{L}$ aliquots of these cell suspensions were stained with 500 $\mu \mathrm{L}$ SYTOX Green (1 $\mu \mathrm{M}$ SYTOX Green stain in Tris- $\mathrm{HCl}(\mathrm{pH}$ 7.5)), along with an unstained control. Stained cells were assayed using a BD FACSCanto II florescence activated cell sorter controlled with BD FACS Diva software (both BD Bioscoences, San Jose, CA). Results were analysed using FlowJo TreeStar data analysis software (http://www.flowjo.com/).

\subsection{Results}

\subsubsection{Resistant mutant generation}

A mutant population was generated using EMS mutagenesis. In order to determine adequate mutagenesis, a viability assay was conducted. Mutagenised cells were compared to an unmutagenised control on SC Agar plates. The mutant population generated showed a sufficient attrition rate to demonstrate a good level of mutagenesis had been achieved, and was suitable for carrying forward to the screening stage.

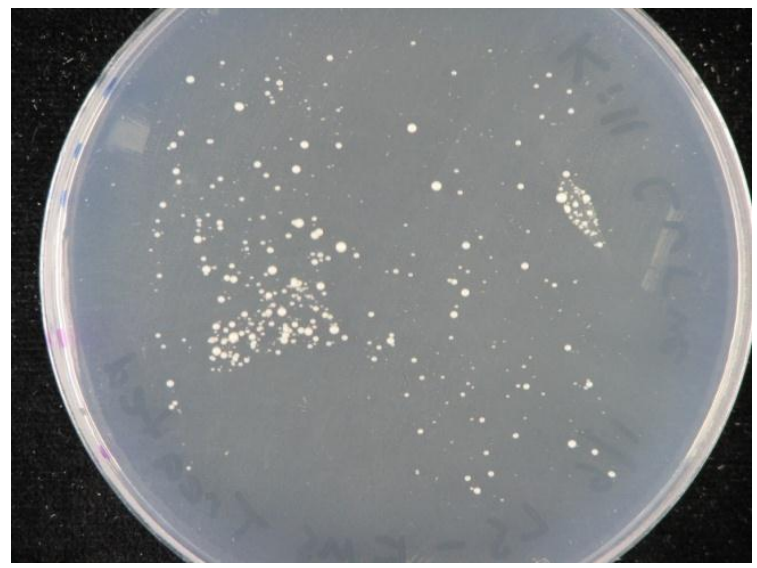

Figure 4.5 Mutagenised cells treated with 3\% EMS for 1 h. Note the number of petite cells in this population. This phenotype is likely caused by a deleterious mutation causing cells to grow slower or smaller, or the loss of mitochodria. There are a sufficient number of healthy colonies at this level of mutagenesis to proceed with screening.

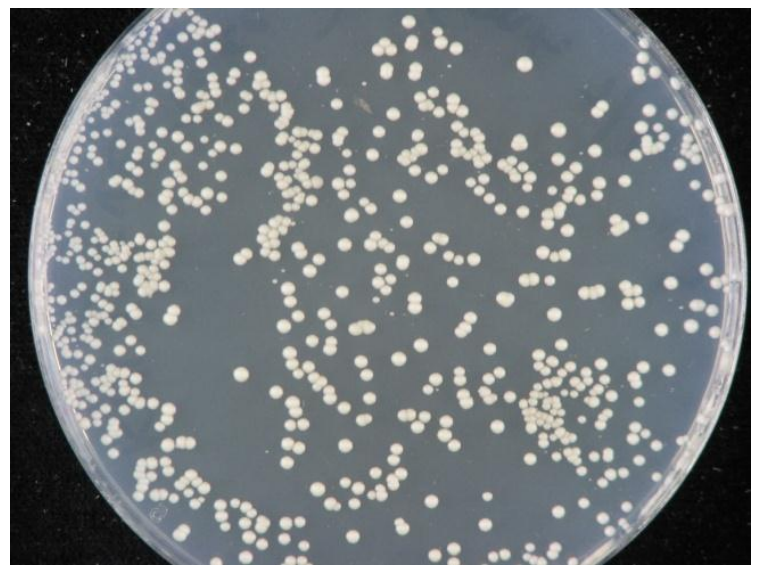

Figure 4.6 Unmutagenised control population. Large healthy colonies show no sign a petite phenotype. There is about a $30 \%$ attrition rate in the mutagenised population compared to this control sample. 


\subsubsection{FC-592 resistance screening}

The resistant mutant population was washed, grown for $4 \mathrm{~h}$ to express mutant proteins, and screened for resistance to FC-592 on SC FC-592 Agar at a range of concentrations. After growth, 5 mutants were identified growing on FC-592 concentrations which proved inhibitory to unmutagenised control cells. These resistant colonies were harvested and designated as YCG432, YCG433, YCG434, YCG435 and YCG436.
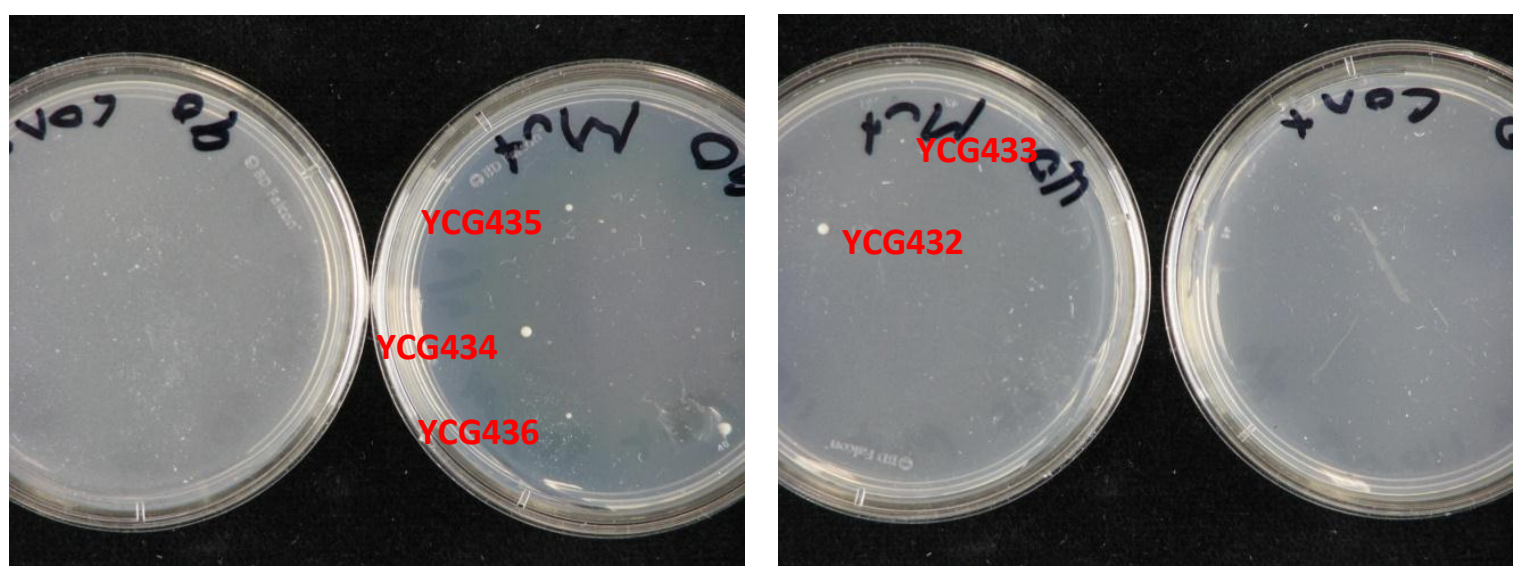

Figure 4.7 Mutagenised cells screened on $90 \mu \mathrm{M}$ FC-592 (r). Three viable colonies were obtained. YCG436 shows a petite phenotype, whilst YCG434 and YCG435 colonies are larger. No cells grew from the unmutagenised population (I) screened at the same concentration.
Figure 4.8 Mutagenised cells screened on $110 \mu \mathrm{M}$ FC592 (r). Two viable colonies were obtained, one considerably larger than the other. This could be because of a deleterious mutation on the part of YCG433, or a mutation conferring only partial resistance, whose phenotype is significantly ablated by FC- 592 .

These five mutants were obtained on FC-592 concentrations of $90 \mu \mathrm{M}$ and $110 \mu \mathrm{M}$. Larger numbers of viable cells were observed on concentrations lower than this. However, at these lower concentrations several viable cells were also observed on unmutagenised control plates. This calls into question the resistance of cells on these plates - because quantities of unmutagenised cells were able to survive, they clearly do not require a strong level of increased resistance to thrive.

In order to characterise the resistant characteristics of each mutant, a solid state dose response was carried out. Cells were spotted into wells of a 48-well plate containing different concentrations of FC-592. 


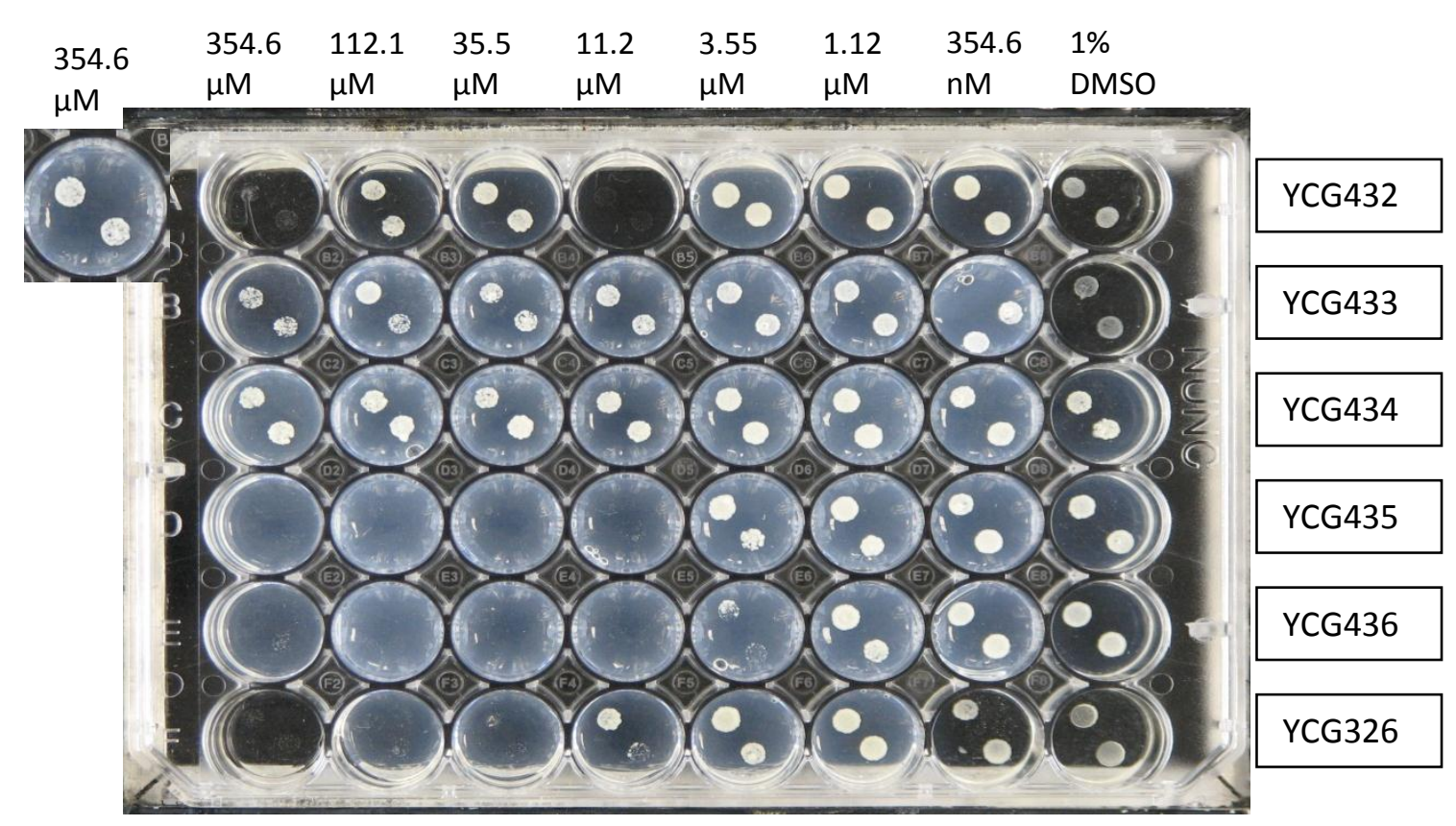

Figure 4.9 Solid state dose response assay of resistant mutants. YCG432, YCG433 and YCG434 proved highly resistant against the compound, whilst YCG435 and YCG436 actually showed decreased resistance. Note that two wells in the top row (YCG432) were desiccated during the incubation process due to uneven quantities of media, and showed no growth. For this reason, the $354.6 \mu \mathrm{M}$ well for YCG432 was repeated (inset).

YCG433 and YCG434 demonstrated strong growth on $354.6 \mu \mathrm{M}$, the highest concentration of FC-592 available. YCG432 demonstrated growth at $112.1 \mu \mathrm{M}$. The well containing $354.6 \mu \mathrm{M}$ FC-592 became desiccated during the first run of this experiment and was repeated. YCG432 grew at this concentration in the repeat experiment. The highest concentration that the background strain YCG326 was able to grow on was $11.2 \mu \mathrm{M}$. Despite growing previously at $90 \mu \mathrm{M}$ FC-592 concentrations in a resistance screen, YCG435 and YCG436 were sensitive to the compound relative to YCG326, being unable to grow at the $11.2 \mu \mathrm{M}$ concentration. YCG436 growth was severely attenuated at the $3.55 \mu \mathrm{M}$ level as well. For this reason, these mutants were discarded from further study.

\subsubsection{Dominance/recessive testing}

Dominant/recessive characteristics of the three remaining mutants (YCG432, YCG433, YCG434) were tested by crossing with a Pdr $\Delta$; His3 $\Delta$ strain and growing resultant diploid strains on $150 \mu \mathrm{M}$ FC-592. If mutations were dominant, the resultant heterozygous diploids would be resistant and grow on the 
test plate. If they were recessive the mutation would be masked by the wild type copy of the gene and the cells would fail to grow.

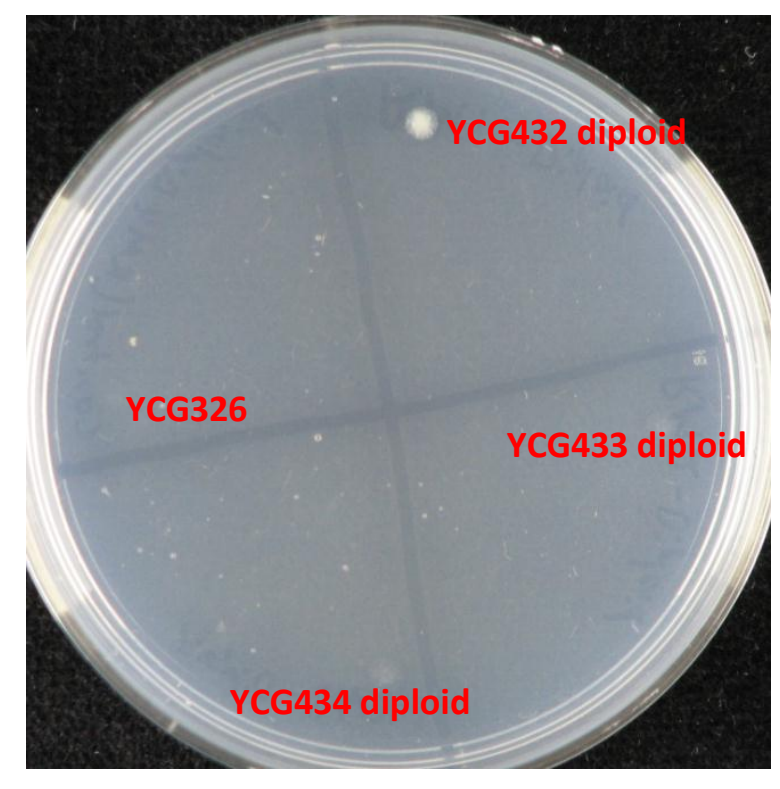

Figure 4.10 Spot assays on $150 \mu \mathrm{M}$ FC-592 of resistant mutants crossed with WT stains to create heterozygous diploids. YCG432 grows strongly, showing a dominant mutation, whereas YCG433 and YCG434 failed to grow, showing a recessive or haplo-insufficiency effect. No growth is seen from an YCG326 control. Note cells did not grow in a visible fashion for any mutant except at the highest density of cells in this spot dilution assay.

Results from this assay indicated that the FC-592 resistance mutation for YCG432 was dominant, whilst those for YCG433 and YCG434 were co-dominant or recessive. As there are several mutant identification strategies that rely on a distinguishable heterozygotic phenotype (such as plasmid library phenotype rescue; Avrahami-Moyal et al. 2012), YCG433 and YCG434 were selected for further study.

\subsubsection{Tetrad analysis}

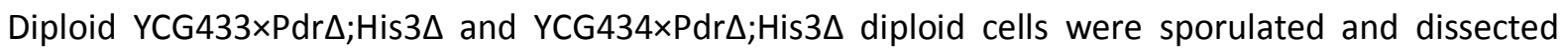
under microscopy to determine whether FC-592 mutations within those strains were caused by a variation at single locus. A single locus genotype would give 2:2 segregation of progeny possessing

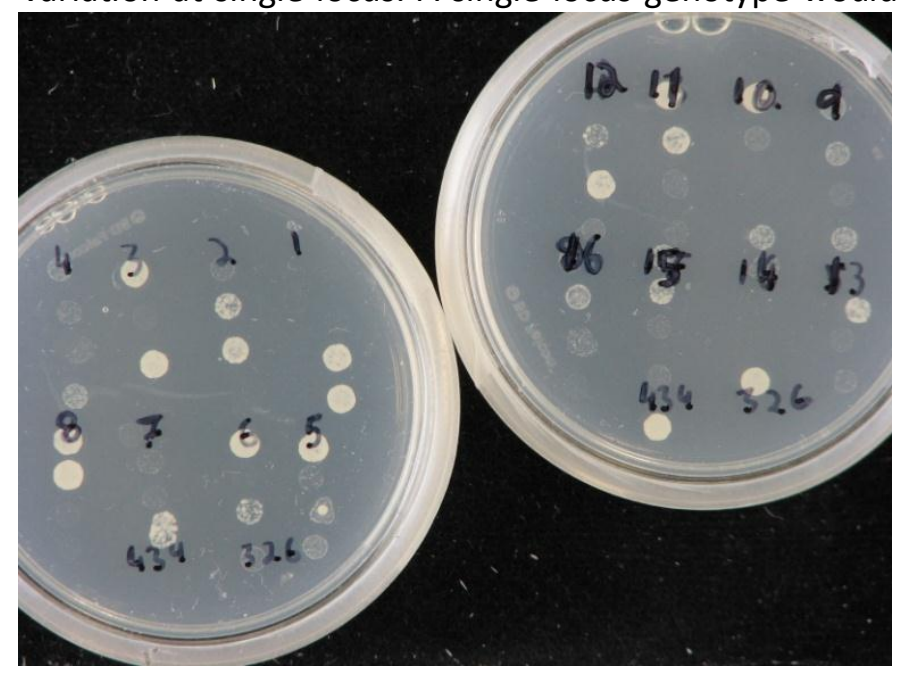

FC-592 resistance. 16 tetrads were dissected and progeny cells transferred to SC media containing $150 \mu \mathrm{M}$ FC-592.

Figure 4.11 Tetrad analysis of resistant mutants. Tetrads

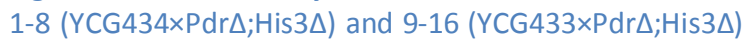
display 2:2 segregation of alleles mediating FC-592 resistance. Of 16 tetrad sets, only \#7 and \#14 do not show a 2:2 pattern of growth on FC-592 media. This could be due to an error in cell transfer to the assay plate. YCG434 haploid and YCG326 cells serve as positive and negative controls respectively. 
Results of tetrad analysis suggest that resistant mutations in YCG433 and YCG434 are due to single locus effects. This is because resistance alleles segregated $2: 2$ in $87.5 \%$ of tetrads tested for each strain, accepting experimental error for the outliers. This result is important for proceeding to synthetic genetic array mapping, as a double locus mutation results in a low proportion of viable progeny. This would result in patchy colonies due to selecting for essentially three mutations: G418 resistance and the two alleles that mediate resistance to the compound of interest.

\subsubsection{Complementation testing}

A complementation test between YCG434 and YCG433 was conducted to determine whether the resistance allele was located in the same functional unit or gene. A Mat A KanR+ cenLeu- YCG433 mutant progeny was crossed with a YCG434 cenLeu+ transformant and tested on a solid state dose response to establish the resultant diploid's resistant properties. If the mutant alleles were located on the same gene, the diploids would possess no wild type copy and the cells would display a resistant phenotype. If the alleles were on differing genes, the wild type copy of each would mask the resistant allele and the cells would display a sensitive phenotype.

$150 \mu \mathrm{M} \quad 135 \mu \mathrm{M} \quad 120 \mu \mathrm{M} \quad 105 \mu \mathrm{M} \quad 90 \mu \mathrm{M} \quad 75 \mu \mathrm{M} \quad$ 1\% DMSO

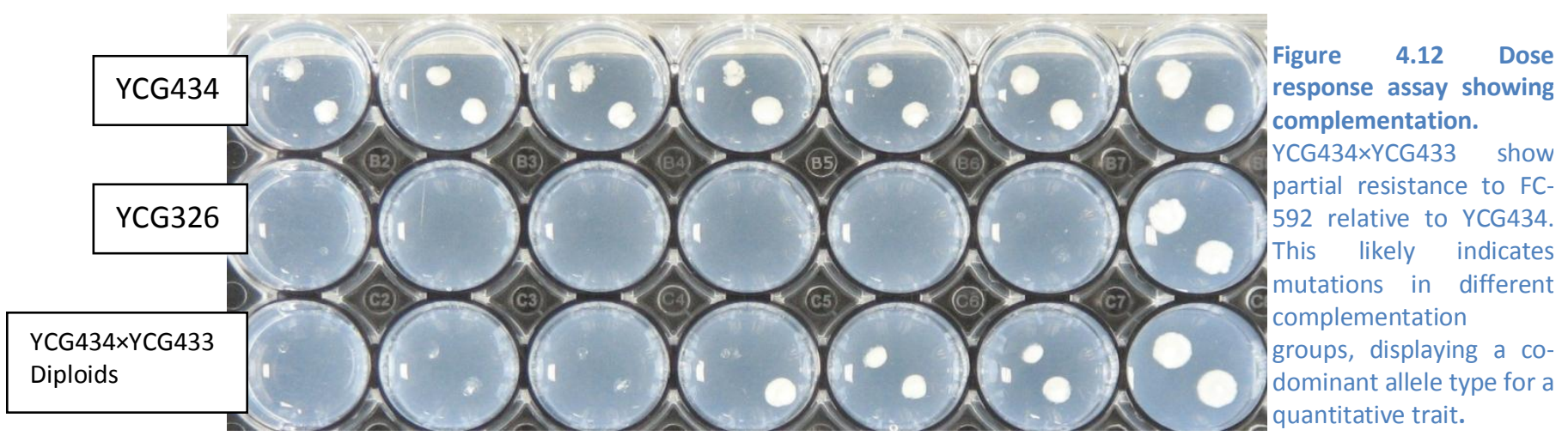

The results of this assay revealed a partially resistant phenotype for the YCG433×YCG434 diploids.

These data are indicative of a quantitative trait with multiple genes contributing to a phenotype. It is possible but unlikely that both resistant mutants represent differing mutant alleles on the same gene (reduction in function mutations), in which case the two differentially defective genes in the diploid may restore some functionality and thus sensitivity. 
More likely is two loss-of-function mutations on different genes which both contribute to FC-592 sensitivity. In this case, haplo-insufficiency means the single functional copy of each gene does not produce enough of a gene product to bring about the wild-type level of sensitivity. In this way, the genes in question would be quantitative trait loci (QTLs), contributing to a quantitative characteristic - namely FC-592 resistance.

\subsubsection{Multidrug resistance analysis}

In order to determine whether the resistance mutations in YCG43433 and YCG434 conferred general resistance or specific resistance to FC-592 compared to the background strain YCG326, dose response experiments were conducted utilising cycloheximide, rapamycin, hygromycin B, fluconazole and nystatin, as well as FC-592. If resistance to these known inhibitory compounds were observed in the resistant mutants, it would suggest a general mechanism of resistance not giving insight into the mode of action for FC-592.

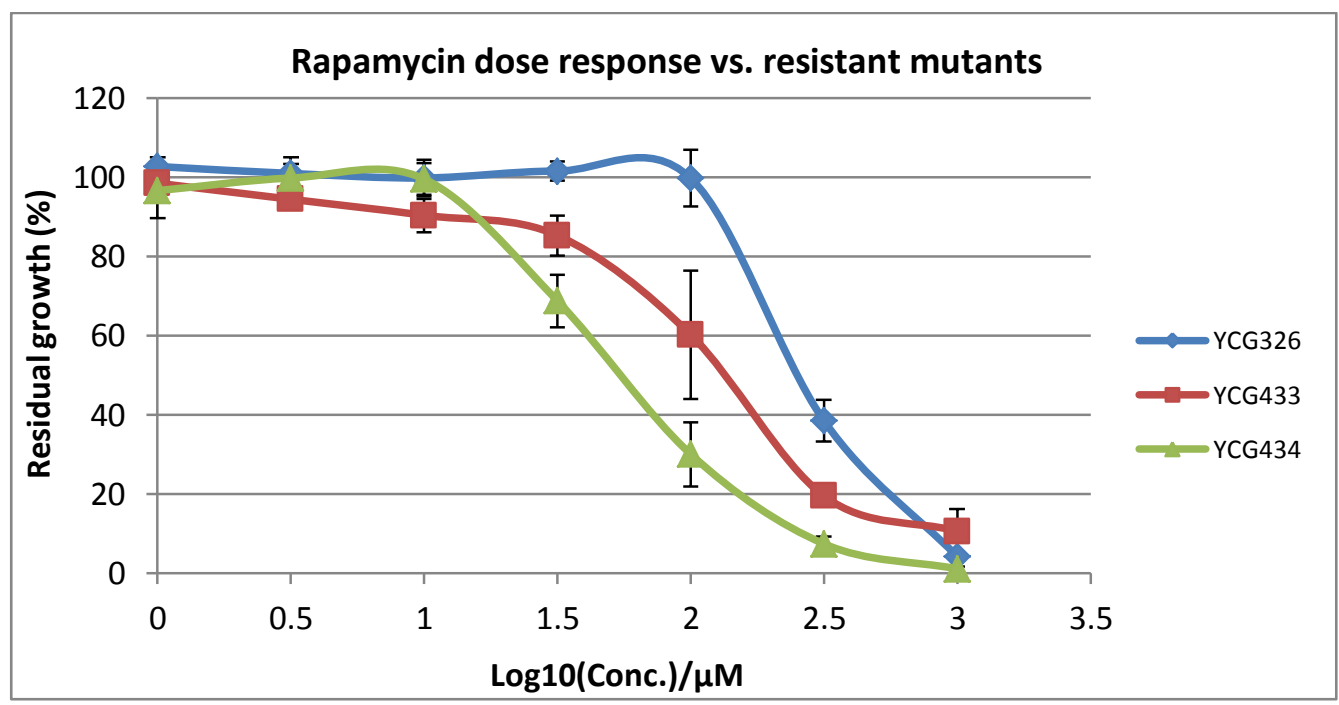



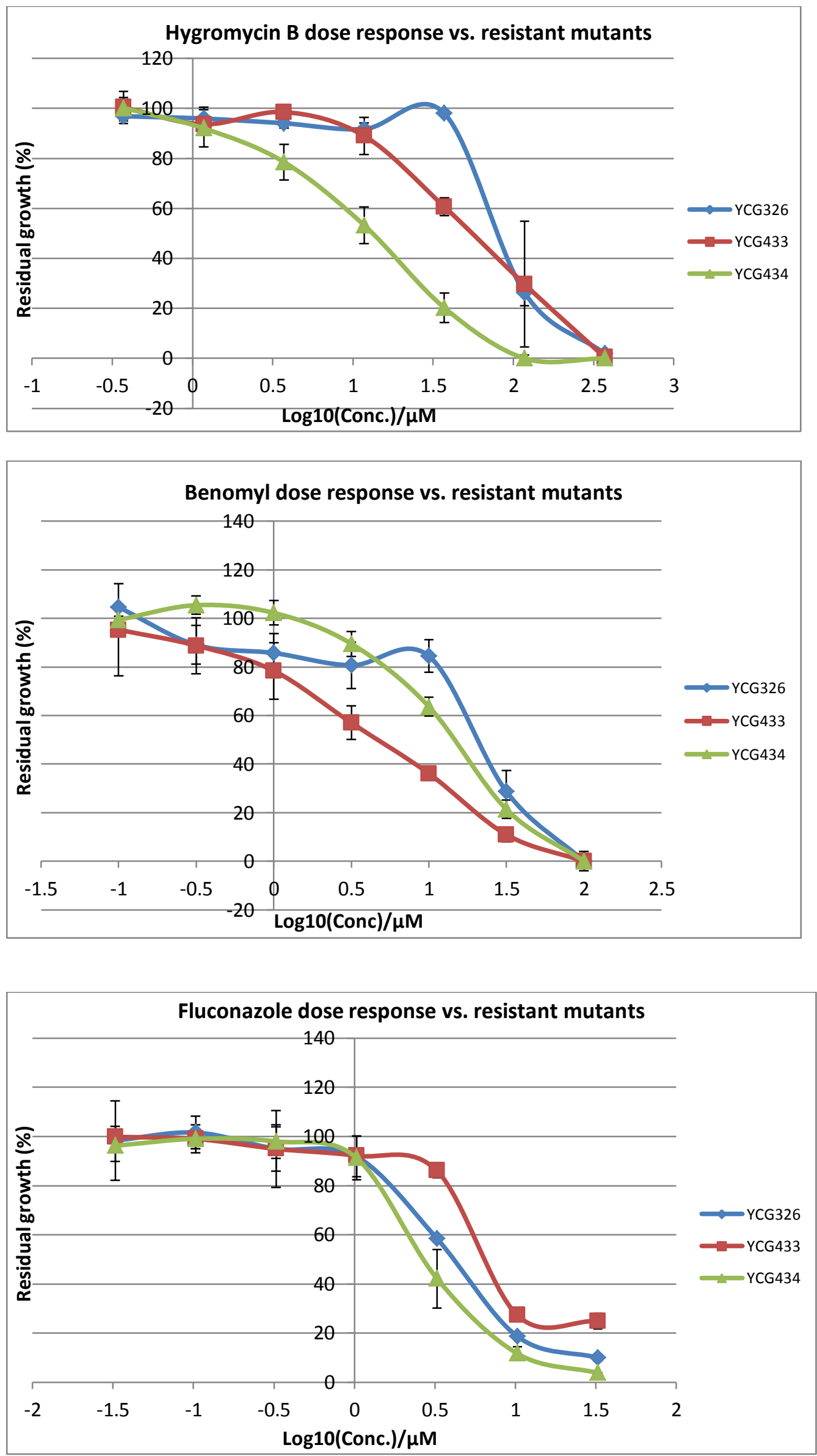

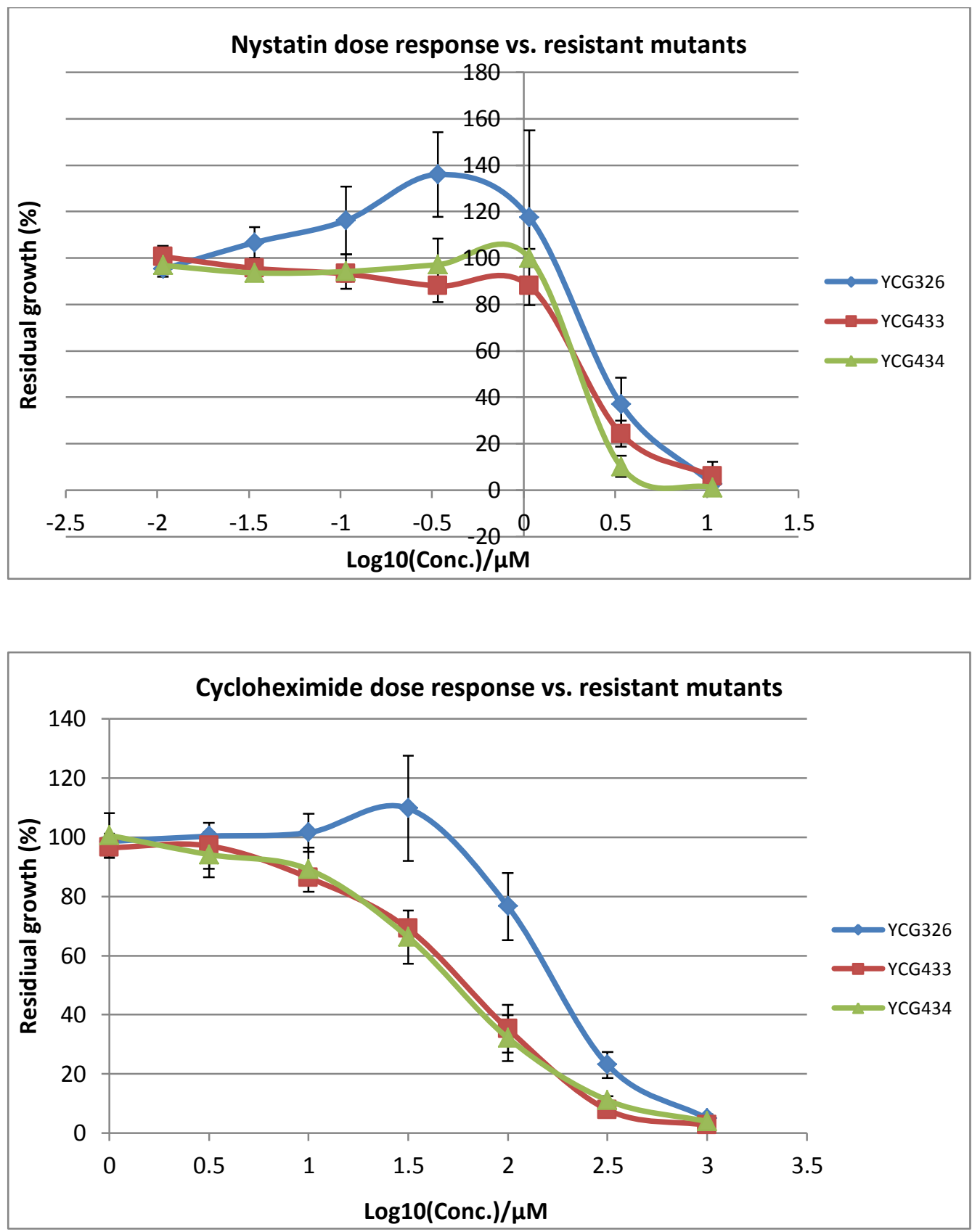


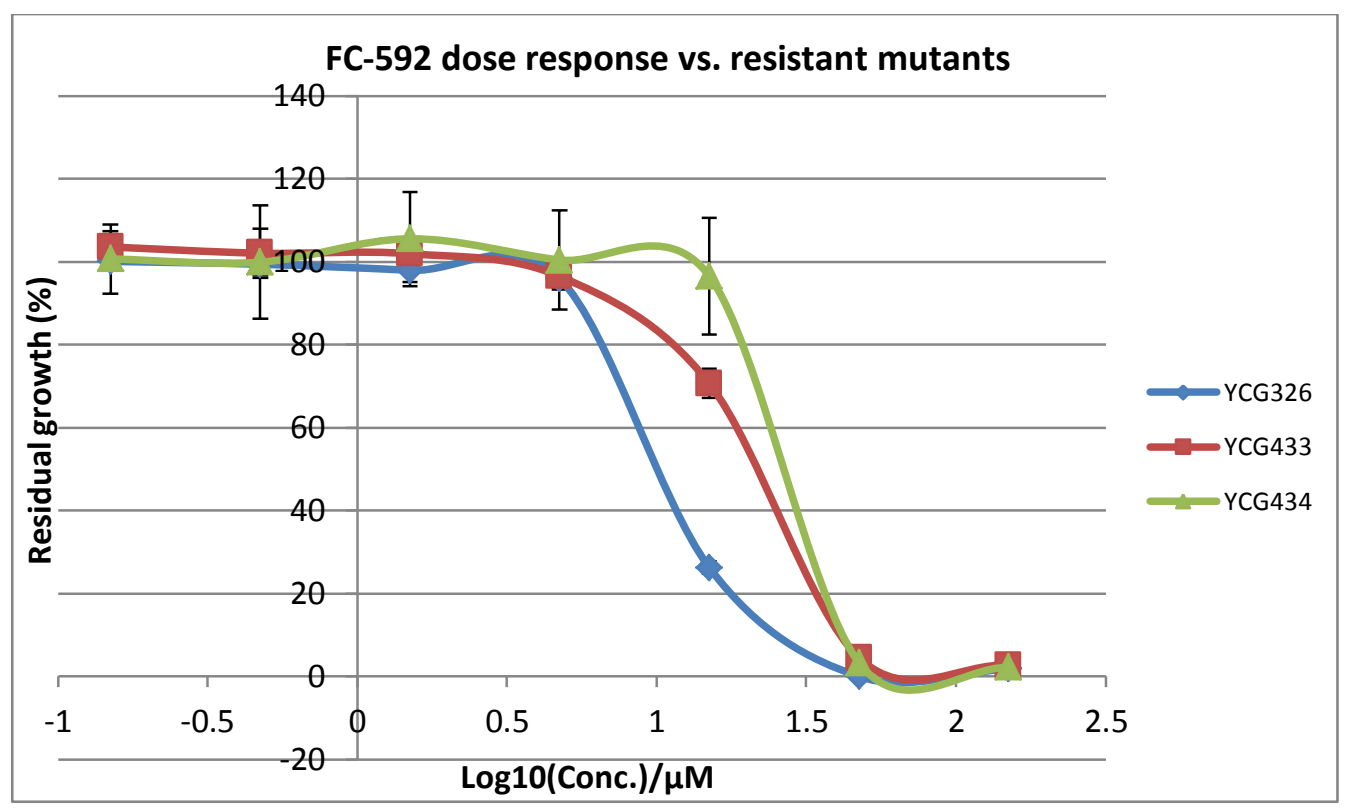

Figure 4.13 Multidrug resistance analysis of the FC-592 resistant strains YCG433 and YCG434. The resistance strains show specific resistance to FC-592, being able to grow in a higher concentration than a control strain, YCG326. However, these strains show comparative sensitivity, relative to YCG326, to rapamycin, cycloheximide, hygromycin B, benomyl and nystatin. YCG433 shows some limited resistance to fluconazole relative to YCG326, whilst YCG434 is comparatively sensitive to this compound as well.

The FC-592 resistant mutants were shown to be specifically sensitive to that compound in a multidrug resistance assay. Apart from fluconazole, neither strain exhibited increased viability at higher doses of the tested compounds compared to the background strain YCG326. In addition, YCG434 showed no increased resistance to fluconazole, and YCG433 showed only marginally increased resistance. These data provide strong evidence that resistant mutations observed in YCG433 and YCG434 do not cause general resistance to a wide range of inhibitory compounds, although the sample size in this case is limited.

\subsubsection{Growth rate of resistant mutants}

Growth rate of resistant mutants was determined to ensure they were close enough to wild type growth rate to proceed on with. This assay revealed that YCG434 grew at very close to the same rate as the background strain control. However, YCG433 displayed a significant growth defect in comparison to that strain. It is important for the SGAM experiment that the query strain grows at a WT rate, as otherwise the resistant mutants will be outcompeted during the initial selection phases of the experiment, resulting in an underrepresentation of resistant alleles in the final selection 
sample, and thus an underestimation of resistance. For this reason, YCG434 alone was selected to carry on to the SGAM phase of the experiment.

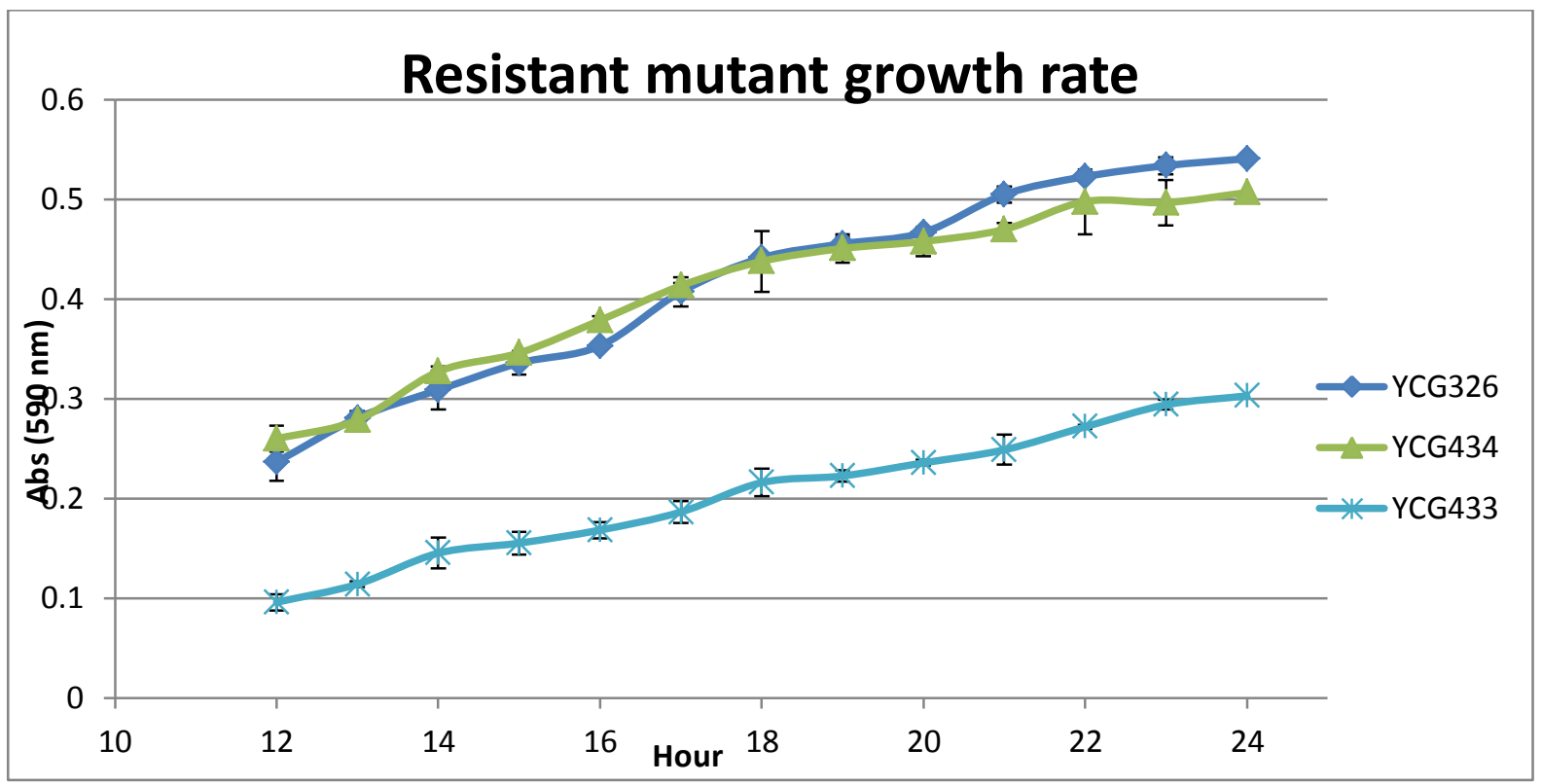

Figure 4.14 Time series growth assay. The growth rate of resistant mutants YCG433 and YCG434 was assayed in non-drug conditions against a background strain control, which grows at a WT rate. Grown from a similar starting titre, YCG433 displayed a severely attenuated growth rate, making it inappropriate for use in future experiments. YCG434 displayed little or no growth defect compared to the WT and was selected for use in SGAM experiment. YCG434 and YCG326 were only just reaching saturation after $24 \mathrm{~h}$, longer time than expected. This may have been due to a low starting titre.

\subsubsection{Synthetic genetic array mapping (SGAM) - proof of concept}

In order to prove the utility of the SGAM technique, a pilot experiment was conducted using the cycloheximide resistant strain YCG191 and a cycloheximide/G418 double mutant selection. Only strains which possess copies of the mutant RPL28 gene and a KanR resistance cassette from the Boone DMA would be viable under this selection. 


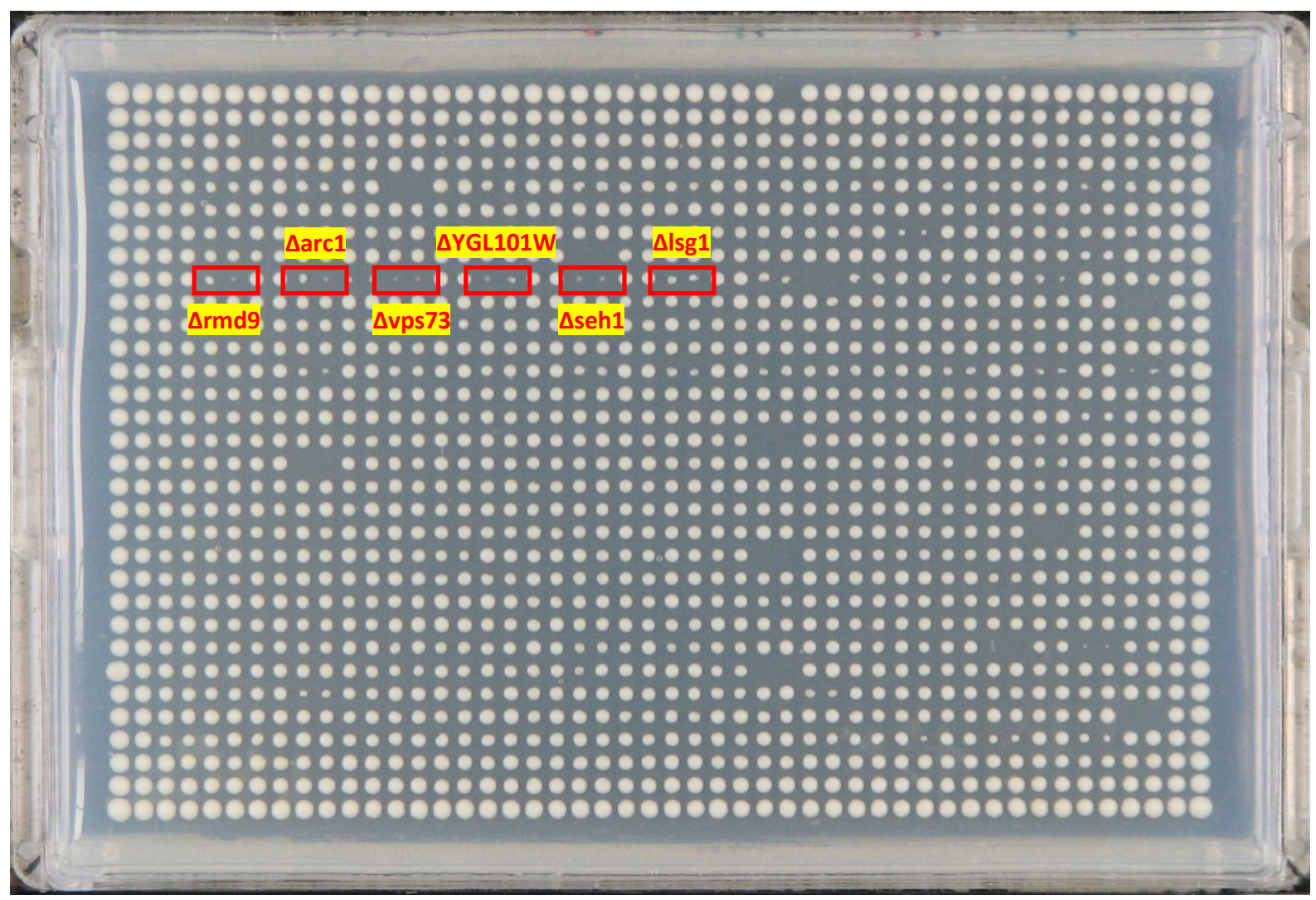

Figure 4.15 A linkage disequilibrium pattern is observed on plate three of the SGAM. These 'holes' in growth on the plate represent deletion strains from the Boone DMA, which when crossed with the cycloheximide (CHX) resistant mutant YCG191, are inviable under the double mutant selection of $200 \mu \mathrm{g} \mathrm{mL}^{-1} \mathrm{G} 418$ and $10 \mu \mathrm{M} \mathrm{CHX}$. Homologous recombination means that DMA strains carrying a KanR cassette on genes close to the site of $\mathrm{CHX}$ resistance (the ribosome protein encoding gene RPL28) cannot carry both resistance mutations, and are therefore inviable under double selection conditions. Note that RPL28 is an essential gene and does not appear as a deletion mutant in the DMA. Therefore the holes observed in the above SGAM plate represent genes that surround the RPL28 locus on chromosome VII within the yeast genome. These genes all occur within 7000 bp of RPL28.

The results from the pilot SGAM experiment confirmed this as a viable technique. The DMA strains $\Delta \mathrm{rmd9}, \Delta \operatorname{arc1}, \Delta \mathrm{vps73}, \Delta \mathrm{YGL101W}, \Delta$ seh1 and $\Delta$ lsg1 were identified in a linkage disequilibrium pattern illustrating the genomic location of the cycloheximide resistance mutation RPL28-ChxR. RPL28 is surrounded by these genes on chromosome VII in the S. cerevisiae genome. It is especially noteworthy that this technique is able to identify a mutation on an essential gene, which are usually invisible to assays incorporating the haploid DMA. 


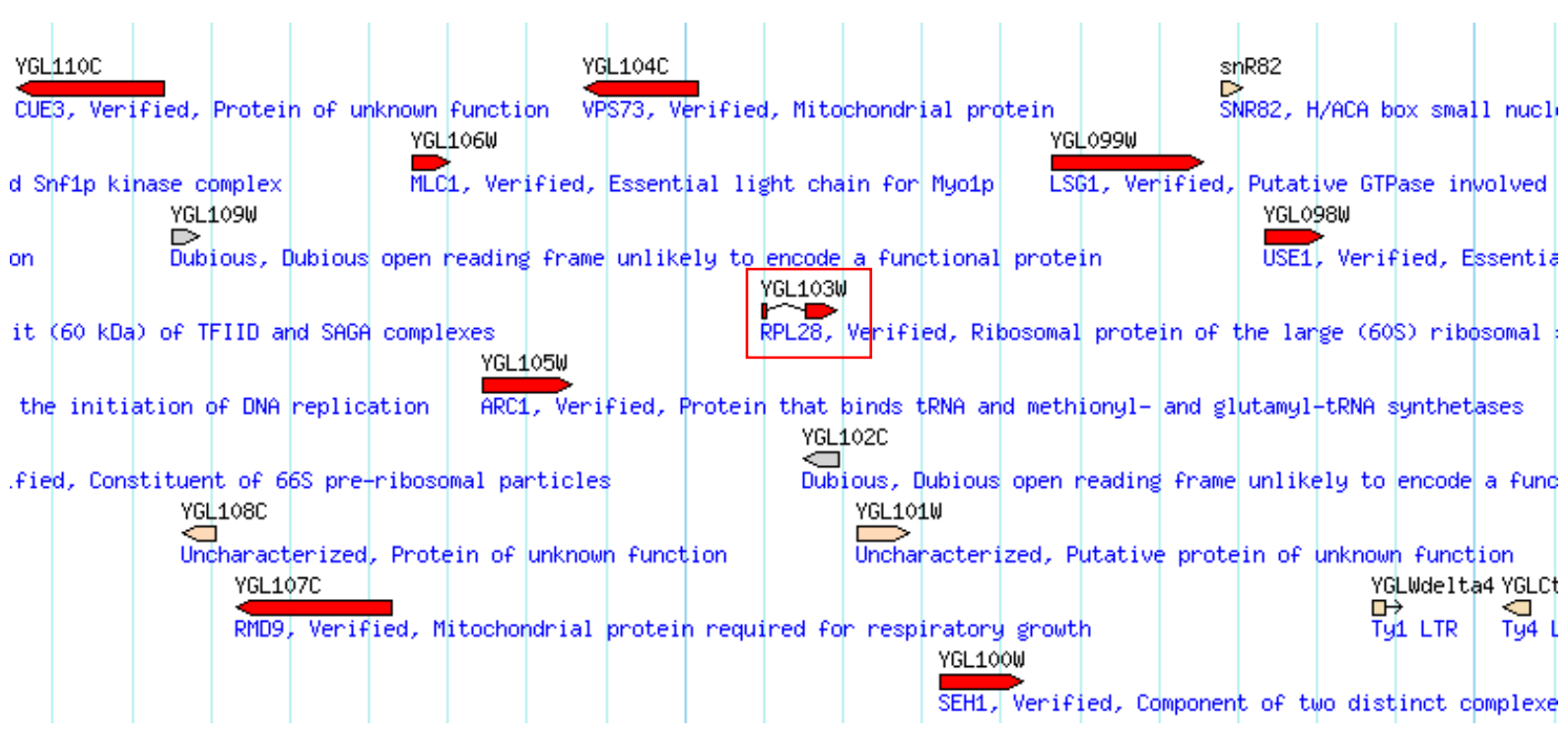

Figure 4.16 The genomic location of RPL28. RMD9, ARC1, VPS9, YGL101W, SEH1 and LSG1 are the nearest non-essential neighbours on chromosome VII. The improbability of recombination events during meiosis amongst neighbours this close means that progeny are unable to carry copies of both KanR and RPL28-ChxR mutations. Source: yeastgenome.org.

\subsubsection{YCG434 FC-592 resistant mutant - Synthetic genetic array mapping}

YCG434 was selected as a query strain for the SGAM experiment. A synthetic genetic array was created using this stain and the Boone DMA. During construction of double mutants for the array, patchy growth was noticed on several steps. For this reason, a high copy strategy was used whereby each plate was pinned twice to maximise the amount of cells that were transferred to each new selection. However, this proved insufficient to create sufficient growth in the final selection step.

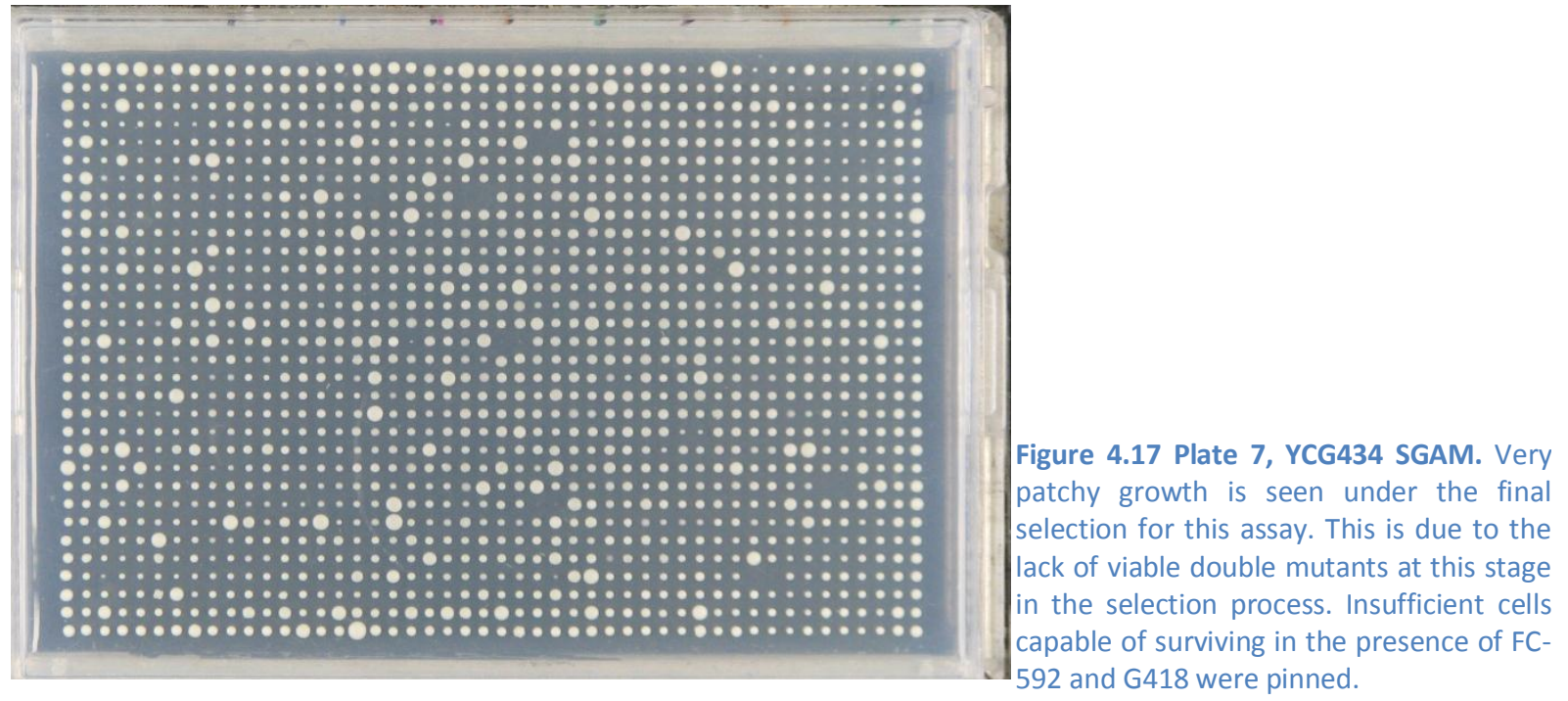


Figure 4.18 Resistance phenotypes. This image shows a resistant (growth) phenotype (red), and a 00 sensitive (non-growth phenotype (blue). Substantial amounts of cells are seen even in the non-growth phenotype because a large amount were pinned from the previous selection in an attempt to overcome - 1 patchy growth problems, an effort that proved in vain. A true growth 'hole', established at an earlier selection, is shown in yellow. This hole represents the array position of PDR3, which is unable to survive under selective conditions due to the URA3 auxotrophic marker.

Growth was too patchy after the final selection of this experiment to make any conclusions about the genomic location of the FC-592 resistance mediating locus in YCG434. Colonies displaying a growth phenotype provided inconsistent coverage of the array and made identification of any linkage pattern impossible.

\subsubsection{Cell cycle analysis by flow cytometry}

The effects on the cell cycle of FC-592 were analysed using flow cytometry. Treated cells were fixed and stained with SYTOX Green before analysis with a fluorescence activated cell sorter. This assay determines the proportion of the cell population at each phase of the cell cycle by measuring SYTOX stained DNA content of each cell.

Control

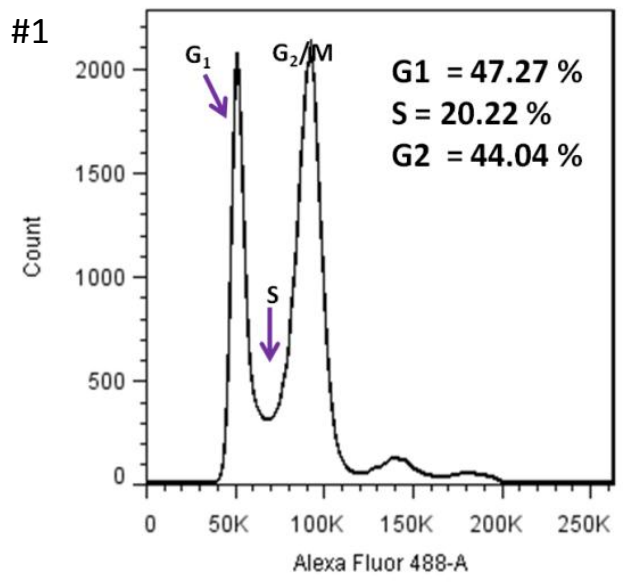

Control

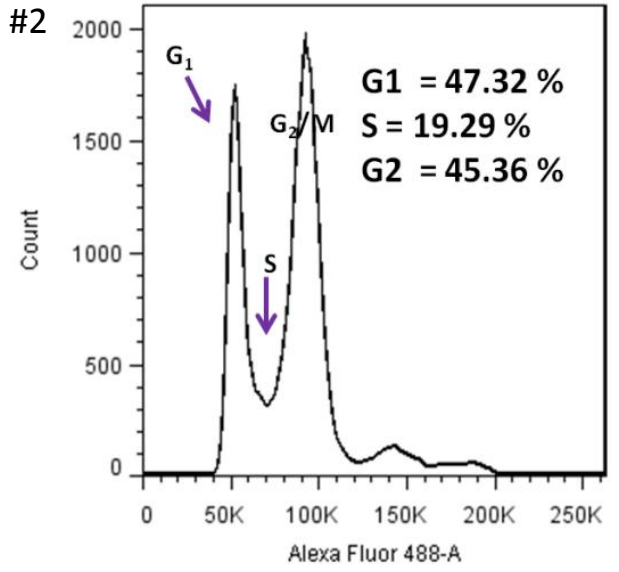

Treated

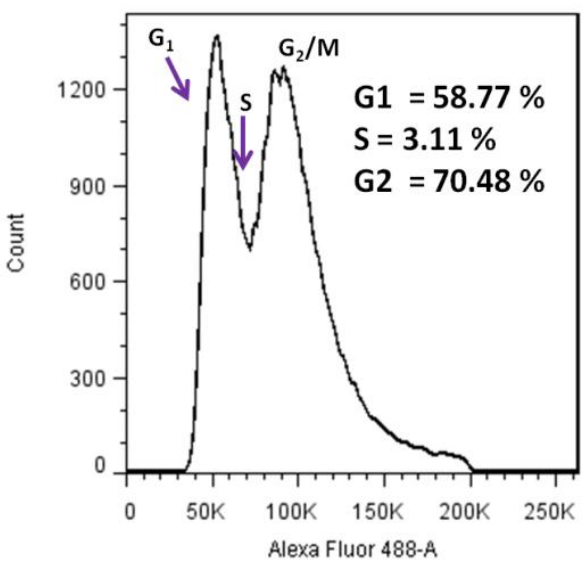

Treated

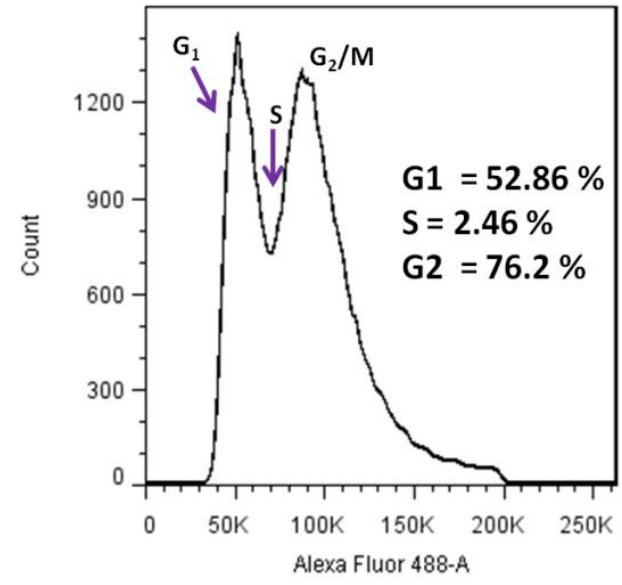




\section{Control}

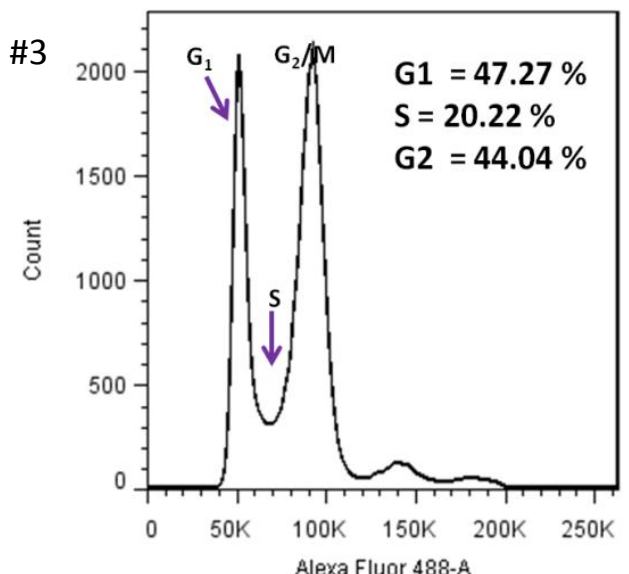

Treated

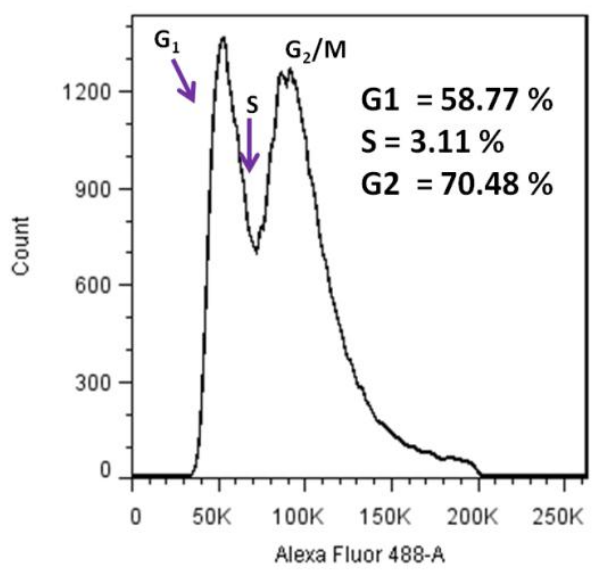

Figure 4.19 Three replicates of FC-592 treated cells compared to untreated cells in a flow cytometry cell cycle experiment. Replicates were run on separate days using different cultures of YCG326, explaining the differences seen between each replicate. Within each replicate however, the proportion of cells in $\mathrm{G}_{1}$ phase fell whilst the proportion of cells in $G_{2} / M$ phase increased substantially. In replicate $\# 1$, $S$ phase cells increased slightly as a proportion, whilst in the other two replicates the proportion of $\mathrm{S}$ phase cell declined sharply. This difference is likely caused by differences in the starting cultures. These data indicate a $G_{2} / M$ phase block caused by FC-592.

Results from this experiment indicated a likely $\mathrm{G}_{2} / \mathrm{M}$ phase block upon treatment with $\mathrm{FC}-592$. Proportion of cells in this phase increased an average $22.67 \%$ (STDEV=3.28\%). This suggests that the mode of action of FC-592 prevents the cell proceeding through cytokenesis, at some step in late interphase or early mitosis. There is also a notable decline in S phase cells in two of three replicates. Whilst possibly an artefact or experimental error, this decline could indicate an effect of FC-592 on the $G_{1}$ checkpoint control mechanism. 


\section{Chapter 4 Discussion}

\subsubsection{Resistant mutant generation}

Mechanisms of resistance frequently give insight into mode-of-action studies for novel compounds (Kahan et al. 1974; Davidse, 1986). This strategy was used in an attempt to identify a gene or genes mediating sensitivity to FC-592 in a manner which would provide insight into its mode-of-action and/or target. Resistant mutants were successfully generated for this compound. This involved recruiting a mutant population from EMS mutagenised cells, screening them for resistance on FC592 containing media, and defining some of the characteristics of these mutations through tetrad analysis.

A mutagenised population was produced via the EMS protocol, displaying a high-quality range of diversity in growth phenotypes when grown on SC media. For example, many small colonies were seen, indicating a deleterious mutation that slowed growth rate, whilst many normal sized colonies were also seen. In addition, kill rate of $\sim 30 \%$ was achieved in the mutegenised population relative to the unmutageised control. These factors are a good indication that a level of mutagenesis was introduced to the population that struck a balance between introducing sufficient variation into the population and survivability of cells (Winston, 2008).

Screening revealed five resistant mutants in an initial screen, three of which proved to have sustainable resistance on follow up dose response assays. It is unknown why the remaining two mutants grew in the initial screen, but showed reduced resistance relative to the wild type in the dose response, but could be due to some revertant mechanism.

A dominance/recessiveness test revealed that one was dominant, whilst two mutants were recessive, when the tested trait was grown at $150 \mu \mathrm{M}$ FC-592. Further testing revealed a likely semidominant mechanism in the latter two, where mutations in YCG433 and YCG434 both contributed to FC-592 resistance. This makes sense in view of resistance as a quantitative trait: this is commonly the 
case with respect to resistance to antiproliferatives (Sen \& Ferdig, 2004). The semi-dominant (functionally recessive when tested at $150 \mu \mathrm{M}$ ) mutants were selected, due to their possible utility in future experiments such as genomic library plasmid rescue assays.

The recessive/semi-dominant nature of these alleles makes it likely that they are a result of loss-offunction or partial loss-of-function mutations (Lodish et al. 2000). This has implications for the mechanism of resistance, which will be discussed later in this chapter.

Tetrad analysis revealed that resistance traits for both YCG433 and YCG434 were mediated by single locus mutations. This is because they segregated $2: 2$ in meiotic progeny produced by mating with a (mating type switched) background strain query. This is important for the success of SGAM and makes it easier to identify the mechanism of resistance involved in each of these cases, as it presumably doesn't involve complex interactions between multiple resistant loci.

On the other hand, complementation analysis revealed that the resistance mediating alleles in the two strains were likely located on different genes. This obviously means that there are multiple mechanisms of resistance involved, although they could affect the same process or pathway. The upshot of this is that it will be harder to specify a specific mode of action from these resistance strains: it is apparent that resistance is not mediated by a single target. Of course, based on previous results from this project, it seems unlikely that FC-592 affected a specific target in any case. As discussed in Chapters $2 \& 3$, a broad based mode-of-action is far more likely. It is likely therefore that there are more genes mediating resistance, which if discovered could allow a greater understanding of the compound's multiple targets.

Finally, a multidrug resistance assay was conducted to ensure that the mutations withinYCG433 and YCG434 did not mediate general resistance to antiproliferatives, for example by up-regulating efflux pumps. This is critical because such a mutation would give no insight into the mechanism of the compound. The only indication of cross resistance in any of these assays was slightly increased 
resistance for YCG433 to the triazole antifungal fluconazole. Fluconazole bears little structural similarity to FC-592, so it is unknown how this cross resistance is caused, especially considering YCG433 did not display any such resistance to any of the other compounds assayed. It is possible that this result was due to experimental error or some artefact of the testing procedure, as the extra resistance relative to the background strain in this case was minimal.

\subsubsection{Synthetic genetic array mapping}

The two components for mode-of-action studies utilising resistant mutants include the generation and screening of the mutants themselves, and the identification and characterisation of the mechanism of resistance. It is this second component which gives insight into the possible mode-ofaction of the compound, and is frequently the more difficult part of the process. Although mutants resistant to FC-592 were successfully generated for this experiment, the synthetic genetic array

:०००000.00000

0.000000000

0.00 .0000 .00$.

locus proved ineffective. Since a pilot experiment using a

100.00000000000000

0.00000000000 O०:-000000000

Figure 4.20 Growth phenotype colonies were few and far between. This proved insufficient to identify a failure of this experiment is likely mediated by the resistance linkage pattern on any of the plates in this assay.

mapping (SGAM) strategy used to identify the resistance-causing

previously identified cycloheximide resistant mutant proved

successful, the protocol itself is clearly not at fault. Instead, the mutation itself.

A growth phenotype was seen on selective media containing $150 \mu \mathrm{M} \mathrm{FC}-592$. However, rather than the even growth phenotype that is normally seen in SGA experiments (Baryshnikova et al. 2010), the growth phenotype was seen in only a few colonies. This obviously did not allow a linkage disequilibrium pattern to be detected in a way which would reveal the resistance mediating locus in the genome: most regions were too sparsely covered by growing colonies to detect the distinctive inhibition zones in neighbouring colonies. 
Significant growth was seen outside of the 'growth' phenotype colonies. These are identified as smaller colonies on the SGA final selection plates. The two phenotypes are however quite distinctive: there is a clear difference between the larger, growing colonies and the smaller, nongrowth phenotypes. Partial growth in the 'non-growth' phenotype colonies is likely due to several factors.

In an effort to alleviate patchy growth seen at earlier selection stages, and to achieve a higher ratio of 'growth' colonies, larger amounts of yeast were pinned to the plate during the SGA protocol. This meant that a sizeable amount of cells in each colony were transferred to the plate before the growth assay was even started. It is feasible that cells in these larger starting colonies were able to achieve a small level of growth even if they did possess the requisite alleles to thrive on the selective media on which they were being grown. Since some of these cells would have been situated away from direct contact with the surface of the drug-containing media, it is possible that they were in essence shielded from the compound and received a lesser dose. These cells could still have obtained the nutrients required for limited growth from the breakdown of dead yeast cells within their own colony, or through development of filamentous growth (Zaman et al. 2008; Jin et al. 2008). Therefore, these cells were able to grow but at a much slower rate than those colonies which displayed a 'growth' phenotype.

These 'growth' cells are proposed to be those cells which were the object of the SGAM all along: meiotic progeny of the original cross containing both the FC-592 resistance mutation from the resistant strain YCG434 and the KanR cassette from the Boone DMA. As already noted, these cells were not common enough amongst the colonies in toto to produce a viable array experiment. There are several possible reasons for this stemming from one basic cause.

There were not enough double mutant cells at the penultimate stage of selection to provide good growth characteristics in all colonies. This means that the FC-592 resistance mutation was far rarer in the combined array population than would be expected for a single locus mutation, wherein we 
would expect fully half the cells to exhibit this trait. A multi locus mutation could explain this discrepancy. In this case, rather than segregating 2:2, the resistance trait would segregate 1:3 (for a two locus mediated trait), or 1:7 (for a three locus mediated trait). In these circumstances there would be fewer resistant cells in the population, and therefore fewer viable cells transferred onto the selective media. This can result in patchy growth (Tong \& Boone, 2007), as many colonies do not possess enough viable cells to produce a visible growth phenotype relative to other colonies.

However, tetrad analysis showed that FC-592 resistance segregated 2:2 amongst the meiotic progeny of YCG434 (and YCG433 for that matter). Therefore it is possible to rule out a multi-locus mediated trait as a cause of SGAM failure. The resistance trait should have been present in $50 \%$ of meiotic progeny, meaning they should have been just as abundant in relative terms as KanR resistant cells, or any of the other selectable traits which worked as predicted in earlier selection steps.

An alternative theory for why FC-592 resistant cells were present in insufficient numbers is that the mutation causes sensitivity to other compounds, which were used as selectable markers during the SGA process, such as nourseothricin, geneticin, canavanine, thialysine. Indeed there is some evidence that YCG434 is generally sensitive to a range of other compounds: in a multidrug sensitivity assay, YCG434 was significantly more sensitive than a background strain control in response to cycloheximide, fluconazole, hygromycin B and rapamycin. For this reason, the resistance mutation may have been selected against long before the final selection step in the SGAM experiment.

This is a plausible explanation. However, nourseothricin (inactivates NAT by monoacetylation of ßamino group of the ß-lysine residue) and geneticin (through action of aminoglycoside 3'phosphotransferase) are protected against by resistance cassettes which provide a very high level of resistance to these compounds (Van Driessche et al. 2005; Goldstein \& McCusker, 1999; Davies \& Jimenez, 1980; Sohn et al, 1999). Therefore, a general sensitivity caused by the FC-592 resistance mutation seems unlikely to cause growth defect amongst the resistant mutants in this manner. 
In the case of canavanine and thialysine, resistance is mediated by mating type (Broach et al, 1979; Tong \& Boone, 2006). Mat $\alpha$ cells activate the arginine permease CAN1, which allows cellular uptake of the L-arginine analogue canavanine, which is incorporated into biosynthesis thereby producing structurally aberrant proteins which cannot perform their native functions (Rosenthal, 1986). This function is absent in can1D::STEpr-Sp_his5 Mat a cells, such as the SGA strains used in ths experiment.

Similarly, the toxic lysine analogue thialysine is excluded from Mat a lyp1 $\Delta$ cells, for which the lysine permease LYP1 is deleted. Barring breakdown of the cellular mechanisms which exclude these drugs in the absence of CAN1 and LYP1, it is highly unlikely that weakening the cells against a general chemical attack, as is supposed with YCG434, would explain sensitivity to canavanine and thialysine in Mat a cells.

A third plausible reason for the lack of resistance mutation carrying cells at the penultimate selection stage is general slow growth in these mutants. If this were the case, the mutant progeny would be out-competed by their wild type peers at every stage of selection. In this circumstance, even a mild growth defect could add up over the course of the experiment so that the population of resistant mutants by final selection would be drastically reduced. In order to try to circumvent this, a growth rate assay was conducted with YCG434 and YCG433. YCG434 displayed WT levels of growth and at the time was judged to be sufficient for continuation into the SGAM protocol. However, reconsideration was necessary after the failure in growth at the final selection stage.

The growth rate assay which determined the near WT fitness of YCG434 was conducted in liquid media, whilst the SGAM was entirely grown on agar based plates. Cellular metabolism is known in many cases to be different in solid or liquid based media (ZoBell, 1943). This is especially so relating to nutrient gathering, where solid media can trigger filamentous growth and a change in gene expression profiles (Prinz et al. 2004). For this reason, wild type and FC-592 resistant segregants were examined for growth defects via tetrad analysis. 


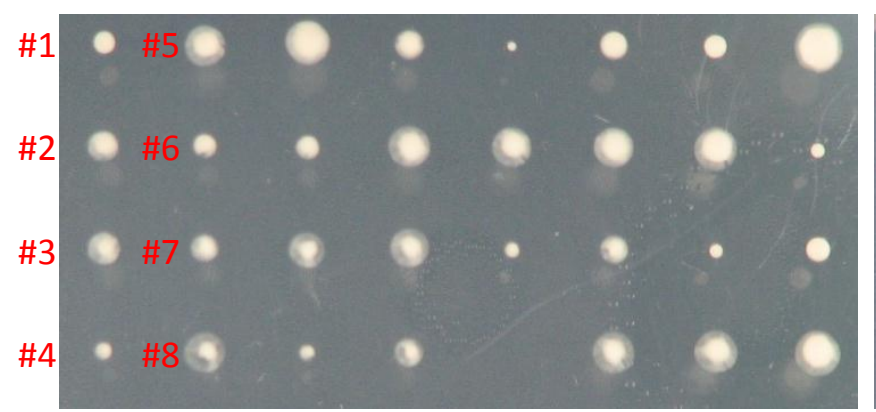

Figure 4.21 Tetrads growing on SC media. Tetrads seem to Figu This seems to indicate a growth mediating single locus resistant mutant displaying a growth phenotype in mutation.

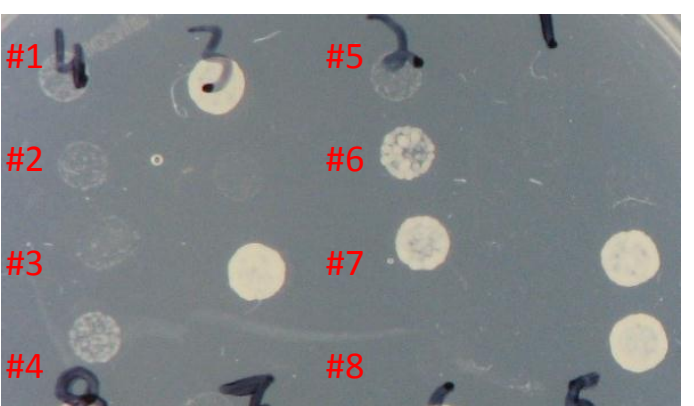
the FC-592 containing media also displayed a small colony phenotype in the tetrad plate. This is compelling evidence that the resistance allele also mediates a small growth phenotype in non-selective media.

Small colony phenotypes observed in tetrads grown on non-selective media were congruent with FC592 resistance in tetrads grown on selective media. This evidence very strongly suggests that despite the fact that YCG434 grew at or near WT rates in liquid media, it was a slow grower in agar based assays. The possibility is that the FC-592 resistance mediating mutation affects a gene that is important for growth on solid media, for example an invasive growth associated protein such as the MEK kinase Ste11p. This would account for the difference in growth on differing media types, but it is unclear how it would mediate resistance to FC-592. Xu et al. (2002), and Anastasia et al. (2012) suggest a link between $G_{2} / M$ arrest, cell size and secretory genes. The regulation of cell cycle mediating secretion is mediated by the exocyst complex, which consists of eight subunits, SEC3, SEC5, SEC8, SEC10, SEC15, EXO70 and EXO84. Assuming FC-592 activity against this pathway, a reduction-of-function mutation here may mediate resistance to FC-592 activity against the complex, whilst also causing a small cell or slow growth phenotype.

YCG434 possessing a slow growth phenotype on agar neatly explains the failure of the SGAM experiment as performed: progeny from the original cross that carried the resistance mutation were 
strongly outcompeted by WT cells, meaning they were present in insufficient quantities to achieve coverage of the genome. Only a few rare examples possessed a significant number of these double mutant cells and displayed a growth phenotype in the final selection.

An alternate strategy that would counter this problem would be to grow each of the stages of the SGAM experiment on FC-592 containing media. This would counter any advantage WT cells hold over the resistant mutants by selecting for the resistance mutation at each step. In this scenario, the final selection would be performed with G418, selecting for KanR containing double mutants in a way that would reveal the sought after linkage disequilibrium pattern. However, a phenomenal quantity of the compound would be necessary to achieve this, far more than has ever been synthesised. This would clearly only be feasible if an effective and efficient synthesis were devised for the compound, enabling large quantities to be produced.

A less compound hungry strategy might be to adapt the SGAM protocol for use entirely in liquid based assays. This would use a plate handling robot to transfer cells between wells in 384-well cell culture plates. Each selection would be performed as for the Agar based assay, except in liquid media, allowing the resistant mutants to compete on an equal footing. An additional advantage of this approach would be the fact that cells are more sensitive to FC-592 in liquid media, meaning less of the compound would be required for a given number of assays. A literature search reveals that this strategy has not been tried before, and therefore is speculative. However it would seem that it is an approach worth trying. Proving the feasibility of such an assay could have utility for future applications.

\subsubsection{Common mechanisms of resistance}

There are several common mechanisms of resistance in resistant mutants which could give insight into the possible causes of FC-592 resistance in YCG433 and YCG434. These include loss-offunction/gain-of-function in a gene required to activate/deactivate the bioactive compound; disruption of the binding site in the compound's target protein, or a gene that affects the target 
protein; up-regulation of defensive cellular responses such as the PDR drug efflux network; and modification of uptake mechanisms such as permeases that mediate the ability of the compound to penetrate the cell.

Mechanisms of resistance mediated by alteration of activators/deactivators, rely on disrupting a mode of bioactive chemical activity which requires modification to an active form. The inhibitory compound is inactive in its original form, but is metabolised into a toxic metabolite by cellular processes. This type of compound is commonly known as a prodrug, and has no effect on the cell if the bioactivation mechanisms are disrupted (Blanchard, 1996). Relatedly, organisms can develop defences that will degrade the bioactive compound into an inactive form, rendering it harmless (Morrissey \& Osbourn, 1999), either through direct action of the mutant protein on the compound, or through upstream effects that lead to an up-regulation of the degratory enzymes. FC-592 is a structurally simple compound with no chemical moieties which immediately suggest a mechanism for activation to a more functional form, nor breakdown to a less active form. However, these remain distinct possibilities and are some of the more plausible mechanisms of resistance to this compound in YCG433 and YCG434.

In circumstances where the binding site of the target protein or complex is modified, affinity of the inhibitory agent for that binding site is reduced (Weisblum, 1995), lowering the potential for inhibition of the function of that protein or complex. When the native activity of that target remains, it may be able to carry out its biological function without being disrupted. This is most commonly seen when the compound has high affinity for a very specific binding site (Pryor et al. 2002). In cases where a more general mechanism is responsible for the activity of the compound, modification of just one target is unlikely to seriously ablate that activity. Since FC-592 is predicted to have a multitarget mechanism, it seems unlikely that direct modification of a specific target is responsible for the resistant phenotypes seen in YCG433 and YCG434. 
A common mechanism of drug resistance in yeast is the system of drug efflux pumps mediated by the PDR network, which has been previously described in this thesis. Mutations causing upregulation of genes within this network have been frequently implicated in inhibitory compound resistance (Tsai et al. 2010; Vandeputte et al. 2011). However, this mechanism is less likely as a mediator of resistance to FC-592 in these resistant mutants for a pair of reasons. Firstly, the PDR network in these cells has been severely attenuated by deletion of the PDR1 and PDR3 transcription factors. Therefore, there is little PDR response left to up-regulate in this particular circumstance. In addition, evidence from the multidrug dose response assay suggests that YCG433 and YCG434 are specifically resistant to FC-592, whereas the PDR network mediates resistance to a broad range of compounds.

Modification of compound uptake is frequently implicated as a mediator for resistance across a range of bioactives and in numerous species (Maser et al. 1999; Schnappinger \& Hillen, 1996; Hall, 2002; Bray et al, 2003). Numerous transmembrane proteins are involved in regulating transport of extracellular molecules into the cytosol. A loss-of-function or partial loss-of-function in the transmembrane transporter or permease responsible for uptake of FC-592 would explain the resistance to this compound seen in YCG433 and YCG434. Since many of these proteins are relatively specific for the type of substrate whose uptake they mediate (Abumrad et al. 1999; Wartlick et al. 2004), chances are good that a mutation in this process could specifically cause resistance to FC-592. Slow growth witnessed in liquid and solid media for YCG433 and on agar for YCG434, could be explained by reduced nutrient uptake caused by the loss-of-function in the uptake protein. Taking all evidence into consideration, this seems like the most plausible mechanism for resistance mediation to the compound by the two resistant mutants studied in this project. However, due to the failure of the SGAM assay, this possibility was not confirmed and remains speculative. 


\subsubsection{Cell cycle analysis by flow cytometry}

Taxane diterpenes have a history of clinical use related to cell cycle arrest (Hagiwara \& Sunada, 2004). As a diterpene natural product, FC-592 was therefore analysed for its effect on the cell cycle utilising flow cytometry techniques in a fluorescence activated cell sorter.

The cell cycle encompasses the distinct series of processes and events which lead to cell reproduction (Cooper, 2000). In eukaryotes, these processes are divided into discrete phases in which certain functions must take place before the cell can move on to the next phase of the cycle. At the highest level, the cell division process is divided into the stages of interphase and mitosis. During interphase, the first stage, the cells increase in size, producing the chemicals required for cell division and replicate their genome. In mitosis ( $M$ phase), the chromosomes separate to produce two sets of the genome (anaphase), at which point the cell divides into two daughter cells, a process known as cytokinesis. In the budding yeast S. cerevisiae, this takes the form of a slowly growing bud rather than binary fission.

The key factors that make this process useful to researchers are a series of checkpoints, during which genetic processes assess the cell against a range of criteria to ensure it is competent to proceed to the next stage. The biological purpose of checkpoints is to ensure that damaged or incomplete DNA is not passed on to daughter cells; preserving larger organisms from deleterious tumours and single cell organisms from deficient, evolutionary dead ends. Clinically, this process can be targeted to achieve a desirable effect: most notably the arrest of tumours in rapidly dividing cancer cells (Bharadwaj \& Yu, 2004). Drugs such as paclitaxel (Jordan \& Wilson, 2004) and demecolcine (Yang et al. 2010) stabilise and destabilise microtubule formation respectively, during early mitosis, preventing chromosome separation. This leads to arrest of the cell cycle, and eventual apoptosis.

Cell cycle targeting drugs are tumour non-specific, but take advantage of the fact that cancer cells tend to divide much more rapidly than healthy somatic cells. Since only dividing cells are affected by 
these agents, the effect on tumour cells is much greater than on healthy cells, leading to a therapeutic window.

The flow cytometry cell cycle assay revealed that FC-592 causes a cell cycle block at the $G_{2} / M$ checkpoint. This means that like Paclitaxel, demecolcine and laulimalide, FC-592 causes cell cycle arrest in dividing cells. In a flow cytometry experiment such as the one conducted for this research project, this is apparent as an increase in the proportion of cells with a higher fluorescent index this is analogous to those cells with a higher DNA content, i.e. those cells which have replicated their genome but have not divided yet. This pattern is seen in cells treated with microtubule stabilisingagents peloruside (Hood et al. 2002) and laulimalide (Mooberry et al. 1999).
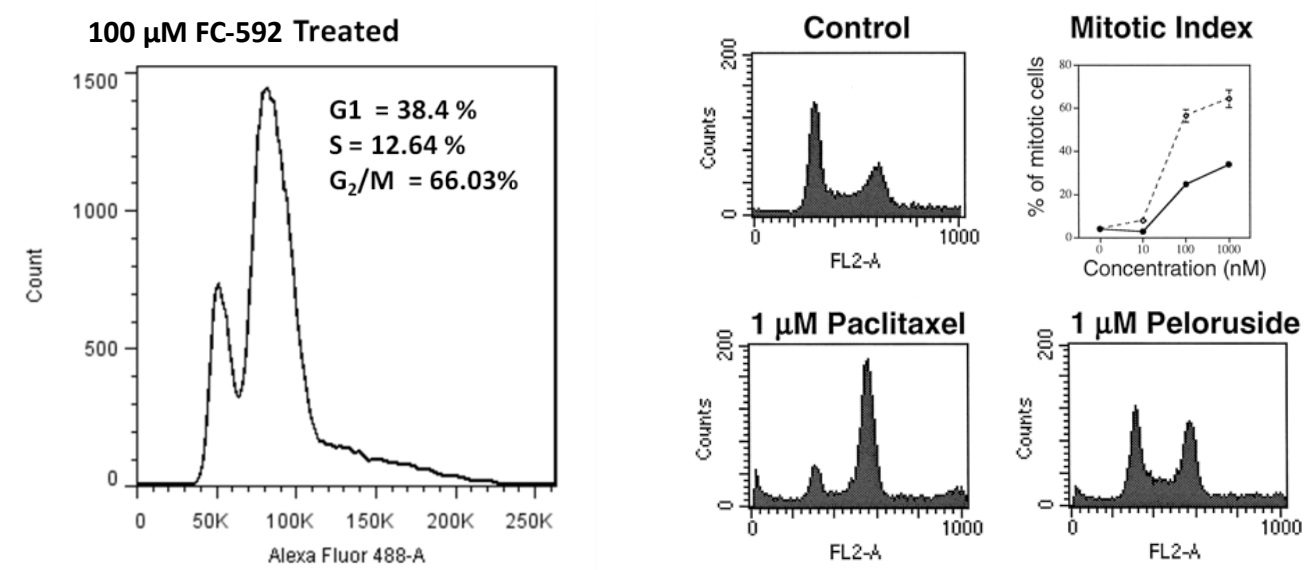

Figure 4.23 Comparison of FC-592 treated cells with previously published assay of paclitaxel and peloruside treated cells. A similar looking increase in $\mathrm{G}_{2} / \mathrm{M}$ phase cells is seen compared to these established microtubule stabilising drugs. It should be noted that the paclitaxel and peloruside treated populations were mammalian tumour cell lines, whilst the FC-592 treated cells were yeast. These model systems have similar cell cycle mechanisms however. Images on right reprinted with permission from AACR: Cancer Research, Hood et al. 2002.

Results obtained from cell cycle analysis demonstrated $\mathrm{G}_{2} / \mathrm{M}$ cell cycle checkpoint block, producing a similar or better looking $\mathrm{G}_{2} / \mathrm{M}$ block profile to two known cell-cycle arresting agents, paclitaxel and peloruside, one of which has a long and storied history as an anti tumour chemotherapeutic.

However, it should be noted that FC-592 treatment was conducted at $100 \mu \mathrm{M}$, whilst the peloruside and paclitaxel assays were conducted at $1 \mu \mathrm{M}$. In addition, the peloruside and paclitaxel assays were conducted against mammalian tumour cells rather than yeast. There is considerable homology 
between these model systems, especially in regards to cell division mechanisms (Cooper, 2000), and thus the relevance of comparisons is likely. However, this comparison shouldn't be treated as quanitative as it is unknown how different levels of cell cycle arrest would affect the profile of the respective cell types seen in the assays. Simply put, it is probably safe to say that FC-592 produces a cell cycle blockage as seen in the other two figures, but these numbers shouldn't be treated as quantitative until further work can be done, comparing like with like.

This result has intriguing implications for FC-592. Although previous work has suggested that the compound is likely to be a non-specific binder, and therefore unlikely to provide the specificity required in a chemotherapeutic, it would be of considerable interest to explore the mechanism of cell cycle arrest. This is especially so considering the rarity of cytoskeletal targeting drugs like paclitaxel (Jordan \& Leslie, 2004).

It is unlikely that FC-592 has similar activity to the likes of paclitaxel through binding to the taxol binding site. The reason for this is that the taxol binding site is highly specific (Buey et al. 2004) to its

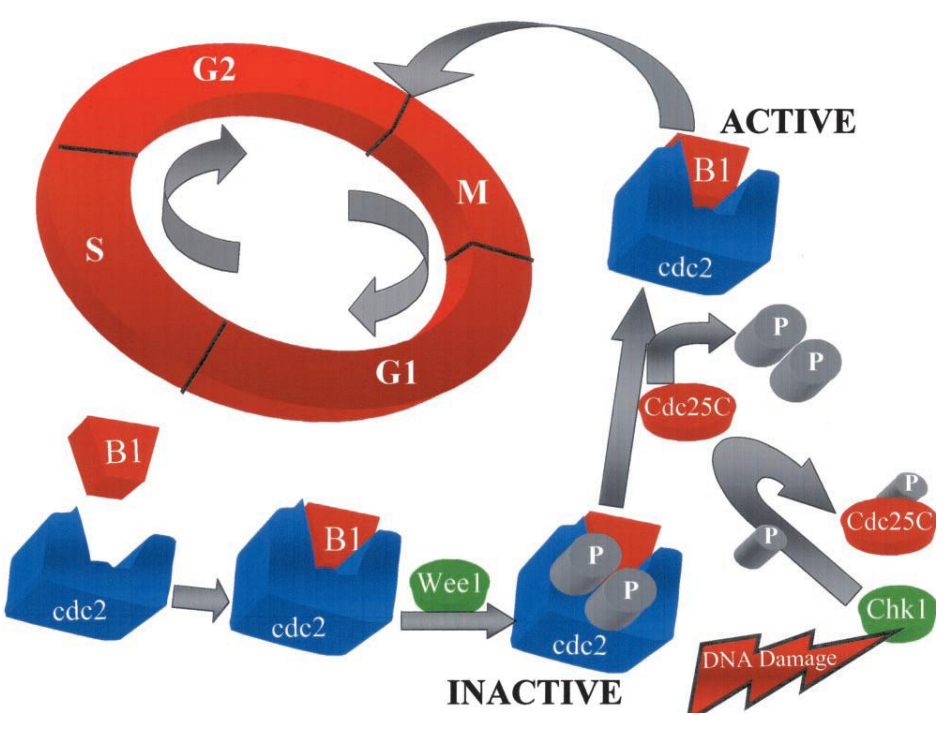

Figure 4.24 Mammalian component of the Cdk pathway regulating entry to mitosis. Inhibition at several binding sites in this pathway could lead to deactivation of the Cdc2-B1 complex which is required for mitosis to proceed. This would result in a $\mathrm{G} 2 / \mathrm{M}$ phase cell cycle block such as that observed experimentally in FC-592 treated cells. A conserved pathway analogous to this exists in yeast. Reprinted with permission from AACR: Clinical Cancer Research, DiPaola, 2002. ligands, with relatively few known compounds with affinity for the domain. The chances of FC-592 acting in this way therefore seem low. However there are other mechanisms by which FC-592 could be causing cell cycle arrest.

An emerging strategy in drug discovery is targeting of the signalling mechanisms in the cell phase checkpoints (DiPaola, 2002). The 
checkpoint control system is regulated by a family of protein kinases, the cyclin-dependant kinases (known as cell division cycle or CDC genes in yeast), which are in turn controlled by a complex array of proteins, primarily the cyclins. These regulate prgression of the cell cycle through the $\mathrm{G}_{1}$ and $\mathrm{G}_{2}$ checkpoints. For example, in response to DNA damage Chk1p is activated and phosphorylates Pds1p, which in turn inhibits anaphase by binding the separin Esp1p (Jackson et al. 2000; Liu et al. 2000). An analogous mammalian pathway sees Chk1 inactivating Cdc25 in response to DNA damage. This mechanism prevents Cdc25 from activating the Cdc2-B1 complex which is required for progression from $G_{2}$ to $M$ phase (DiPaola, 2002; Jackson et al. 2000). A compound that inhibits certain stages in these processes, such as by competing for binding sites in activating enzymes, could cause a cell cycle arrest. For example flavopiridol, a semi-synthetic derivative of the plant alkaloid rohitukine, is believed to act on the CDC pathway through inhibition of the Cdk-activating enzyme KIN28, docking on Cdk ATP-binding sites and decreasing cyclin D1 levels (Bible \& Kaufmann, 1997; Carlson et al. 1999). In this way it prevents cell cycle progression through selectively inhibiting a key component of the pathway, which in turn leads to the inactivation of other CDC pathway proteins, including the critical Cdc28p, catalytic subunit of the main cyclin-dependent kinase complex (Mendenhall \& Hodge, 1998).

In a similar way, inhibition of targets such as these by FC-592 could cause $\mathrm{G}_{2} / \mathrm{M}$ cell cycle arrest as observed experimentally in the flow cytometry experiment. This would occur even if the compound bound to other cellular targets as a non-specific binder of hydrophobic pockets: it would cause cell cycle arrest in this manner, but it would also cause disruption in other parts of the cell. A more intriguing thought presents itself when it is considered that that the vast majority of CDC genes are essential, and thus not present in the HOP screen assayed in Chapter 3. This means that these strains were not present to show sensitivity in the assay, and thus were not part of the dataset that was analysed for GO term enrichment. It is not possible to say that FC-592 did not target the CDC pathway from the results of the HOP screen, simply because deletion mutants representing these genes were not present to be screened. 
This pathway could be more readily assayed through use of the haploinsufficiency profiling (HIP) screen, a yeast tag microarray experiment that uses heterozygous diploid DMA strains, which contain one functional copy of the query gene. This assay examines the sensitivity mediating properties of gene dosage effects and allows the interrogation of essential genes such as many of those found in the CDC pathway.

The cell cycle associated deletion mutants pin $4 \Delta, \operatorname{cdc} 10 \Delta$ and $\operatorname{cdc} 50 \Delta$ were present in the screen. Pin4p directly participates in $G_{2} / M$ phase progression and becomes inactivated when DNA is damaged (Pike et al. 2004). Cdc10p is a septin ring protein that contributes to cytokinesis (Hanrahan \& Snyder, 2003). The endosomal Cdc50p controls polar growth through modulation of the polarisation of the actin cytoskeleton and cell surface (Misu et al. 2003). Pin4 $\Delta$ represented one of the strongest hits overall by z-score, whilst cdc10 $\Delta$ and cdc50 $\Delta$ presented with z-scores of $\sim-2$, which rank them in the top $2.5 \%$ of recorded z-scores in the HOP screen.

Of slightly less relevance, the spindle-assembly checkpoint complex protein Mad2p presented with a z-score $~ \sim-1.3$ in the FC592 treated HOP screen, which still qualifies it for the top 5\% of z-scores. This protein delays the onset of anaphase in cells with defects in mitotic spindle assembly, and has been implicated in mediating resistance to microtubule binding agents such as benomyl (Tsuchiya et al. 2011; Chen et al. 1999). Compounds registering sensitivity on the HOP screen for this mutant would be expected to affect the cell cycle pathway in some respect. Although FC-592 recorded only a very weak hit against this strain, it does not rule out some activity in this respect.

Whilst this data is by no means definitive, with a very small sample size and a lot of unrelated genes presenting with similar or higher z-scores, it suggests the CDC pathway as a site of action. Most probably, given the likely non-specific nature of FC-592 binding, it would be one of several pathways affected. 


\section{Final discussion}

\subsubsection{Mode of action for FC-592 and FC-888}

The synthetically modified diterpene natural product FC-592 and the synthetically derived haloalkyne ester FC-888 potently inhibit yeast growth. A series of experiments utilising the budding yeast S. cerevisiae provided some evidence towards the biological activity of these compounds. Initial assessment revealed that the inhibitory potency of neither compound is abrogated by changes in $\mathrm{pH}$ conditions in common media types. FC-592 proved to be a substrate for the PDR network of efflux pumps, whilst FC-888 did not. Further experiments were conducted using PDR attenuated strains to take advantage of this fact, in a strategy to reduce compound usage. FC-592 was shown to reversibly inhibit yeast growth, displaying cytostatic activity. On the other hand, FC-888 was shown to kill or irreversibly inhibit cells, in a cytotoxic manner.

In the homozygous profiling (HOP) assay, FC-592 showed weak enrichment for genes associated with glycoprotein biosynthesis and the secretory pathway. A follow-up assay attempting to confirm FC592 activity against these pathways screened for initiation of the unfolded protein response. However, this response was not observed in reaction to FC-592 treatment. FC-888 showed no statistical enrichment in the HOP screen, instead inducing a sensitive reaction in a broad array of deletion mutants representing genes all across the genome.

A synthetic genetic array mapping (SGAM) strategy was attempted to characterise mutants that were resistant to FC-592. Whilst a range of mutants were successfully generated for this experiment, the SGAM failed when the resistant mutation caused slow growth on agar. This led to resistant progeny being outcompeted at selection steps during the construction of the array, and thus not being present in sufficient quantities in the final selection. Therefore, they were unable to generate the distinctive linkage disequilibrium pattern which identifies the target locus in SGAM experiments (Jorgensen et al. 2002). Based on experimental results, it is proposed that the mechanism of resistance observed in the mutants generated in this study, YCG433 and YCG434, is modification of a 
cellular uptake mediator. This explains the specific resistance to the compound, whilst allowing the possibility for a more general mode-of-action for the compound.

A cell cycle analysis experiment using flow cytometry techniques successfully identified a $G_{2} / M$ phase block as a result of FC-592 treatment. It was proposed that this is likely a result of the compound acting on the cell-division cycle $(C D C)$ pathway through interaction with regulatory mechanisms, rather than acting on cytoskeletal elements like actin or microtubules. Alternatively, a link with membrane transport associated cell-cycle control is possible.

Chemistry analysis of the structure of FC-592 and FC-888 suggested that these compounds do not have the structural complexity to be capable of highly specific binding to particular cell targets. Therefore it is likely that each compound employs are more general mechanism, interacting with a wide array of targets.

The evidence produced during this project is indefinite in determining a precise mode-of-action for either compound. Taken together however, some determinations can be suggested. Chemically, FC592 is likely to be a non-specific binder of hydrophobic pockets. It lacks the structural complexity to selectively bind specific targets however, and thus probably inhibits activity in a wide variety of enzymes by non-specifically binding to and blocking their catalytic sites. HOP screen evidence suggests that this particularly affects glycoprotein biosynthesis and the secretory pathway. There are a large range of enzymatic processes in these pathways with ubiquitous distribution throughout the cell, meaning there are a lot of targets to aim at.

Cell-cycle arrest was demonstrated as the likely mechanism of cellular inhibition due to the observed $\mathrm{G}_{2} / \mathrm{M}$ block by FC-592. Like the glycoprotein biosynthesis and the secretory pathways, it is likely that the CDC pathway is one of several being hit by this compound due to its non-selective nature. However, the CDC pathway is obviously such a critical process in cell growth that activity upon it could well be the process by which cell division is shut down. Of course, this process is 
interconnected with many others throughout the cell: effects on these other pathways and processes are likely to have a general weakening effect which impacts on growth as well. The fact that FC-592 proved to be cytostatic indicates that the compound did not irreversibly bind to its targets. Rather it is likely that the compound is able to be removed from its targets, either through enzymatic action or native dissociation, when the cell is removed to fresh, FC-592 free media.

An interesting novel mechanism for control of mitotic entry is proposed by Anastasia et al. (2012). Their data suggest that Wee1-dependent inhibitory phosphorylation of Cdk1 (a mammalian gene that has a yeast homologue, SWE1; Nurse et al, 1976) causes mitotic checkpoint arrest via blocking membrane traffic. Signalling in this pathway is conducted by the protein kinase C (Pkc1), Rho1 GTPase and PP2ACdc55, a specific form of protein phosphatase $2 \mathrm{~A}$, and increases during polar bud growth. The researchers therefore suggest that signalling is dependent on membrane traffic, increasing linearly with delivery of vesicles to the site of bud growth.

This mechanism has relevance to FC-592 because it reconciles proposed activity against transport pathways with the observed mitotic arrest. If confirmed, FC-592 activity against vesicle transport would have downstream anti-mitotic effects due to blocking of this signalling pathway. An emerging area of understanding, further research would be required to develop a testing protocol to assay FC592 against this mechanism, but could involve examining membrane mutants for differential sensitivity to the compound, or an assay which directly assesses membrane integrity (e.g. the propidium iodide exclusion assay described by Macklis \& Madison, 1990).

This process offers an alternative explanation of FC-592 activity to the FC-592 as a non-specific inhibitor theory proposed above. Knowing whether it is simply promiscuous or has a multiple, but still limited, number of targets is important. A QTL type approach, crossing resistant with sensitive mutants and isolating F1 phenotypes may prove illuminating in this respect. It would give an indication about whether the targets of the compound segregate out in a few-loci or many-loci manner. 
FC-888 is likely to be a potent alkylating agent based on its chemistry. It almost certainly irreversibly binds cellular nucleophiles, causing disruption to a host of processes and leading to cell death. It does not have sufficient structural complexity to suggest a selective target. Instead, a wide range of targets is likely. DNA repair mechanisms, associated with mediating resistance to DNA alkylating agents (Simon et al. 2000) were not seen as hits in the HOP assay, indicating that FC-888 most probably does not target DNA more than other cellular nucleophiles.

\subsubsection{Assessment of FC-592 and FC-888 utility}

The utility of FC-592 depends on its characterisation as a non-specific promiscuous inhibitor, or a compound with a multiple but limited range of targets. Evidence for both of these mechanisms was seen in this project and further study will be required to definitively elucidate the answer.

The best use for FC-592 based on current evidence would be in the initiation of cell-cycle arrest, in circumstances when this would be useful experimentally. Cell cycle arrest drugs such as laulimalide tend to be expensive, and a less complex compound such as FC-592 could be easier and cheaper to synthesize. However, the probability is that the compound would affect a range of processes outside of the cell cycle pathways, so it would be inappropriate for experiments attempting to explore these other genetic pathways.

Further study into the precise mechanism by which FC-592 causes arrest may elucidate a clearer utility for the compound as a reagent in this regard. In addition, synthetically modified derivatives of this compound may be able to mimic its mechanism of action on this pathway, whilst increasing specificity. This scenario would lead to promising cell-cycle targeting leads and is worth pursuing.

Additional study into the specific mechanisms by which FC-592 affects glycoprotein biosynthesis and the secretory pathway could also bear fruit. There is potential for the compound to be used as a genetic probe in this sense in assays which require general secretory pathway stress. The mechanism by which the compound affects the pathway would need to be elucidated, perhaps in a genome- 
wide over-expression assay (Luesch et al. 2005). This may help further define any specificity for the pathway as well.

The expected non-specific activity of FC-888 diminishes its potential utility in terms of application as a genetic probe or in some putative therapeutic context. This is because the compound will affect too many cellular processes to be useful in either of these respects: creating too much 'noise' to be relevant in a research setting, and likely causing significant off-target effects in a clinical setting. For this reason it is unlikely that significant applications will be found for this compound in its present form.

Useful applications for FC-888 are therefore unclear. Since it seems to have a general mechanism of toxicity against eukaryotic cells, this compound would not be useful in any clinical context. It has potential as a general antifungal, and its activity against other classes of organisms could be explored to assess its potential as a general antimicrobial compound. There are of course numerous chemical engineering based uses for the compound utilising the cross-linking properties. Non-specifically dimerizing cellular macromolecules could also prove to have interesting biochemical applications, especially in in vitro experiments, although a specific use for this remains unclear.

Perhaps the clearest use of the compound would be in assessing the genetic properties of general alkylating agent resistance. An assay exploring the genetic basis of resistance to a generic alkylating agent such as this could be conducted, again using a genome wide over-expression assay. Genes mediating resistance to cellular alkylation could be identified in such a screen. With little enrichment for DNA repair associated genes, HOP screen results seem to indicate that FC-888 does not act primarily on DNA. Therefore, it must affect other cellular nucleophiles to just as great an extent as nuclear ones. Since most alkylating agents used in a clinical context specifically target the DNA (Warwick, 1963), this offers a somewhat novel biochemical probe: an alkylator of cytosolic nucleophiles. 


\subsubsection{Assessment of experimental strategies and protocols}

The overall strategies and specific protocols used in this project allowed a good deal of evidence to be gathered in a coherent manner, rather than taking stabs in the dark on biochemical based assays. The halo assay allowed bioactive compounds to be identified from a decent sized library of novel synthetics. However, the utility of the halo assay over more traditional liquid media based assays was not proven. The liquid based assay was able to identify more bioactives from the same library, and furthermore, did not have the same issues with gas evolution and buffers causing bubbles in the media. The only advantage of the halo assay is that it is theoretically able to assay a much larger concentration range; i.e. relevant results will be seen at much higher concentrations, because they just cause a larger halo. The liquid assay is unable to distinguish between concentrations that are just inhibitory and those that are inhibitory by many orders of magnitude. In practice however, this was not found to be an issue. All noted bioactives from the halo assay were characterised via dose response for inhibitory potency. This would be just as easy to do with results from the liquid assay.

Limitations observed in the HOP screen based approach were mainly due to the nature of the selected compounds themselves. Neither FC-592 nor FC-888 appeared to mediate a highly selective target or mechanism of action. The assay itself identifies overrepresentation in a sensitive sample of genes involved in a certain process or pathway. It loses resolution when multiple pathways are involved, and a single group of genes no longer presents statistical enrichment. Considering this fact, the assay performed well in providing the evidence it did in the case of FC-592. The HOP screen remains a proven technology; however it is probably more effective in compounds that have a mode-of-action targeted to a single specific pathway.

The only aspect of this project which truly failed was the SGAM characterisation of the resistant mutant YCG434. The resistance mutation itself mediated slow growth on agar, which caused depletion of mutation carrying cells at the final selection step. The assay itself was proven in a cycloheximide resistance based pilot experiment and has been used to successfully identify a target 
and mechanism with another compound (Yibmantasiri et al. 2012). The lesson to take away from this is to ensure WT fitness levels in selected mutants before proceeding with an SGAM program. It should also be remembered that mutants can behave very differently on agar than they do in liquid media, so should always be assayed on the relevant media type where possible.

The UPRE assay was effective and efficient in providing answers about induction of the unfolded protein response. Unfortunately, in this project those answers turned out to be negative, and the assay did not provide positive evidence for the activity of FC-592. It remains an effective method for assaying this biological process however.

The flow cytometry cell-cycle analysis experiment worked well in identifying a $G_{2} / M$ phase block in FC-592 treated cells. This quick and efficient assay is easily repeatable in varying conditions and could form the basis of future investigations into the mitosis arresting activity of the compound. The basis of this strategy could be attempting to rescue mitotic function in various over-expression mutants representing genes in the CDC pathway.

\subsubsection{Assessment of aims and objectives}

The overall aim of this project was to identify novel bioactives from a library of novel organic compounds and characterise their action. In this respect it was largely a success, as a number of inhibitory compounds were identified, and two chosen for further study. FC-592 and FC-888 were assayed with a number of strategies that provided a range of evidence as to their mode-of-action, as described above. Although the precise nature of each compound's mechanism was not identified, general mechanisms were proposed that fit the evidence obtained. Given time and resource constraints, a good level of understanding was achieved.

All aims and objectives presented in Chapter two were achieved. FC-592 and FC-888 were identified as bioactives through the halo assay and characterised with respect to their potency and PDR affinity 
profiles. The halo assay was characterised however as generally inferior to the liquid based inhibition assay, a legacy technique.

The objectives set out in Chapter three involve the successful implementation of the yeast tag microarray HOP screen, which was achieved. Selected hit strains were confirmed via growth assay and several determinations made about the activity of the assayed compounds. The results of this assay were better able to characterise the activity of FC-592 than FC-888. However analytical techniques provide little insight, with low levels of statistical enrichment seen in FC-592 and no statistical enrichment identified in FC-888. This was later assessed as being due the non-pathway specific activity of the compounds clouding the statistical sample with sensitive hits from multiple pathways.

The first objective of chapter four was met, with FC-592 resistant mutants being successfully generated. These were then partially characterised by tetrad analysis, complementation testing, and testing for dominance/recessiveness. In addition, the SGAM strategy was proven to be viable by the successful completion of a pilot experiment utilising the cycloheximide resistant mutant YCG191. However, the identification of the resistant locus on the FC-592 resistant mutant YCG434 failed when cells carrying the resistance allele proved unable to compete with non-resistant cells under non-selective conditions.

A cell-cycle analysis assay was performed in this section of the project. Successful completion of this assay and the identification of a $\mathrm{G}_{2} / \mathrm{M}$ black in FC-592 exceeded the original objectives of this chapter. On balance therefore, this component of the project was a success. 


\section{Future Directions}

There are several avenues of study that remain in the investigation of FC-592 and FC-888. Assays exploring the alkylating nature of FC-888 will confirm its activity in that respect, as well as assessing its viability as a genetic probe for exploration of alkylation resistance. In terms of FC-592 several genomic screens utilising different strategies than the HOP screen could prove efficacious in further defining the activity of this compound against the secretory pathway and cell-cycle pathway. Further, several additional strategies could be attempted to characterise the mechanism of resistance seen in the resistant yeast strains YCG433 and YCG434.

\subsection{Future directions in FC-592}

\subsubsection{Haploinsufficiency profiling assay in FC-592}

The activity of FC-592 against the CDC pathway could be better understood through the use of a diploid haploinsufficiency profiling (HIP) screen (Giaever et al. 2003). This is because most of the genes involved in this pathway are essential to the growth of yeast and thus do not show up in a haploid deletion screen. The diploid screen, consisting of heterozygotic deletion mutants, could identify gene dosage effects in essential genes such as the CDC path, confirming FC-592 activity in this area in a manner that the haploid screen is not able to.

\subsubsection{Genomic over-expression assay in FC-592}

CDC and secretory pathway examination by genomic over-expression assay as with the HIP screen seeks to examine gene dosage effects on the bioactivity of the compound. An array of yeast transformants containing a multi-copy yeast genomic library can be screened for resistance to growth inhibitors such as FC-592, determining genes which mediate resistance in a specific pathway (Luesch et al. 2005). This could provide a high degree of certainty about which processes within the affected pathways are abrogated by the activity of the compound. In addition, multi-copy overexpression of mutant alleles can identify phenotype suppressing effects in either sensitive or resistant mutants (Puig et al. 2002). This allows a still greater characterisation of the genetic 
mechanism of action, but relies on characterisation of a resistance- or sensitivity-mediating mutation.

\subsubsection{GFP-linked proteomics assay in FC-592}

One of the mechanisms by which FC-592 could be affecting activity in both the CDC and secretory pathways, is by blocking some enzymatic process which leads to gene expression of a protein involved in these pathways. This could be examined using the GFP-linked proteomics screen described above. Such a strategy could identify reduced protein expression, which could be bioinformatically linked to its promoters and transcription factors in an attempt to determine the mechanism by which such expression is blocked. This strategy has the additional advantage that possible alterations in sub-cellular location caused by the drug can be used as a phenotype to measure the effect of deletion mutations in other genes.

\subsubsection{Secretory pathway screen in FC-592}

Further dissecting the effects of FC-592 on glycoprotein biosynthesis and the secretory pathway is necessary in determining the usefulness of the compound as a genetic probe in this area. Several assays are available which would assist in this type of characterisation. Recombinant luciferase from Drosophila S2 cells can be used as a reporter for activity of the pathway (Wendler et al. 2010). This would allow efficient assessment of the function of the pathway in combination with a range of treatment conditions involving FC-592 and various yeast mutants.

An alternate strategy utilises western blot techniques to identify conditional effects on glycosylation and the sectretory pathway (Hood-DeGrenier, 2008). This methodology takes advantage of the defined pathway roles of $\mathrm{N}$-linked and O-linked glycosylation (Karp, 2005) to determine where in the pathway disruption occurs. Western blots using an antibody recognising a distinct secreted or cellsurface protein can be used to determine which form of the protein accumulates under a given drug condition. By examining which glycosylated residues the secreted protein is deficient in, these data can reveal the intracellular compartment in which the protein is trapped and therefore the specific 
secretory step that is compromised. A broad organelle specificity assay of glycosylation is available in yeast since the ER form of a glycosylated protein (e.g. secreted invertase) is readily distinguished by SDS PAGE from the Golgi form owing to the high-mannose oligosaccharides added in the latter. This would be effective in confirming the specificity or otherwise of FC-592 for a certain target in the process.

\subsubsection{Characterisation of FC-592 resistance mutations by sequencing}

Whilst resistant mutants were successfully generated in this project, characterisation of the nature of these mutations proved elusive for reasons that have been previously elucidated. Such characterisation remains a valuable source of information however, and could confirm the proposed nature of the mutation as a cellular uptake mediator. Next generation sequencing methods such as Massively Parallel Signature Sequencing (Brenner et al. 2000), and polony sequencing (Shendure et al. 2005) have reduced the cost and efficiency of genomic sequencing experiments (Schuster, 2008). This makes sequencing and subsequent bioinformatic analysis of mutational effects a viable strategy, although the cost still remains significant for a small lab. In addition, multiple SNP mutations are likely to be identified, most of them having no effect. This means a significant amount of 'noise' is likely to be encountered in such an experiment when trying to identify the locus of resistance.

\subsubsection{Characterisation of FC-592 resistance mutations by plasmid rescue}

A plasmid rescue strategy can also be used to identify a locus of interest that mediates restoration of a native phenotype (Epstein \& Cross, 1992). In this methodology, a recessive mutant population is transformed with plasmids containing fragments of genomic DNA. A cell which restores WT activity must contain a gene which suppresses the mutant phenotype, very probably a WT copy of the mutant allele. This suppressor gene is identified through sequencing of PCR amplification of the plasmid. When a comprehensive library of plasmids is used in this fashion, the entire genome can be interrogated for suppressive function (Yan \& Burgess, 2012). 
This strategy requires a recessive mutation that is able to be suppressed by the WT allele. It also requires a deleterious phenotype, so that suppressed mutation can be recovered. Ordinarily a resistance mutation such as that observed in YCG433 and YCG434 would not make a good candidate for this strategy, as a rescued (sensitive) phenotype would fail to grow on a screening assay.

The slow growth phenotype mediated by the resistance mutation seen in these strains (agar based screens only for YCG434) however, makes them viable candidates for a plasmid rescue screen. Treatment with FC-592 would not be required for this - the transformed population would be grown for many generations on ordinary media, with the rationale being that the rescued transformants would out-compete the other cells. These transformants could then be assayed against FC-592 to confirm restoration of WT sensitivity before using a colony PCR and sequencing strategy to identify the gene involved.

\subsubsection{Characterisation of FC-592 resistance mutations by liquid phase SGAM}

Another intriguing possibility for the identification of these resistance mutations would be to adapt the SGAM protocol for use entirely in liquid media, hopefully avoiding the slow-growing phenotype seen in agar based assays. This would be a novel strategy, as it hasn't been attempted before in the published literature. All post sporulation steps in this assay would be transferred via pinning robot to 384-well cell culture plates containing the appropriate liquid media. Plates would be grown as normal and cell growth measured via imaging plate reader. This concept would allow cells carrying the resistance mutation from YCG434 to compete with WT cells and maintain a viable presence in the final population. In theory, the SGAM premise would work just as well in liquid media as on agar. This would however have to be empirically verified.

\subsubsection{Further investigation in cell-cycle analysis}

The cell-cycle was identified as a likely target pathway of FC-592. This holds great interest because of the medical relevance of cell-cycle targeting drugs in tumour suppression (Shapiro \& Harper, 1999). Although it is proposed that FC-592 has a relatively non-specific mode-of-action, this utility is worth 
investigating. A cell-cycle screen against mammalian cells, as described in Mooberry et al. (1999) and Hood et al. (2002), would allow direct comparison of the $G_{2} / M$ phase blocking activity of FC-592 in comparison to more established drugs which act on this pathway. This screen would be an important first step in establishing the relevance of FC-592 activity in this field.

In addition to the genomic screens described above (sections 6.1.1, 6.1.2 \& 6.1.3), a method of defining FC-592's interactions with this pathway would involve the screening for sensitivity of particular mutants. For example the $\beta$-tubulin subunit Tub2p is implicated in the mediation of resistance to microtubule stabilising agents such as benomyl (Thomas et al. 1985). TUB2 attenuated mutants (for example TUB2/tub2 $\Delta$ heterozygotes) would give an indication of the site of action of FC-592 on the CDC pathway if they conferred sensitivity relative to a wild type. This assay would require investigation into candidate $C D C$ associated genes to screen for sensitivity.

Finally, activity against the CDC pathway generally could be confirmed by screening against a known model for $\mathrm{G}_{2} / \mathrm{M}$ phase blockage resistance. For example, a mammalian model overexpressing UBE2C, the $\mathrm{G}_{2} / \mathrm{M}$ phase associated gene known to be overexpressed in various chemotherapeutic resistant solid tumours (Wang et al. 2011), would give a strong indication in this direction. If this model were to prove resistant to the compound, it would confirm suspected activity against this pathway.

\subsection{Future directions in FC-888}

\subsubsection{Alkylating agent assay}

An experimental strategy that has proven effective in characterising alkylation activity seeks to evaluate the candidate compounds in several assays in which alkylating agents would have predictable effects (Mertins et al. 2004). In this study chlorambucil-resistant Walker rat carcinoma cells were assayed for cross-resistance to the test compounds compared with known alkylating agents. In addition, proteomics assays were used to identify increased expression of proteins which 
are associated with DNA repair. This proved effective at assaying the activity of compounds targeting different sites within the DNA molecule and other biologically relevant nucleophiles. Such a strategy could be used to confirm the alkylating properties of FC- 888 .

\subsubsection{GFP-linked proteomic screen in FC-888}

An alternative approach would be utilising a genomic library of GFP-linked proteins (Huh et al. 2003) in an attempt to identify DNA or alkylation repair associated proteins whose expression and localisation is altered under FC-888 treatment. The advantages to this assay include short time frame and low experimental error. However, there may be other genomic effects observed which are not specifically related to DNA or alkylation repair mechanisms: the ramifications are difficult to predict, but this could cloud results. 


\subsubsection{Appendix 1 - IRL compound library data}

\begin{tabular}{|c|c|c|c|c|c|c|c|c|c|c|c|}
\hline Cat. \# & $\begin{array}{c}\text { Mass } \\
(\mathrm{g})\end{array}$ & $\begin{array}{l}\text { Mol. } \\
\text { mass }\end{array}$ & Vol. $(\mu \mathrm{L})$ & $\begin{array}{l}\text { Conc. } \\
\text { (mg/mL) }\end{array}$ & $\begin{array}{l}\text { Conc. } \\
(\mathrm{mM})\end{array}$ & Cat. \# & Mass (g) & $\begin{array}{l}\text { Mol. } \\
\text { mass }\end{array}$ & $\begin{array}{l}\text { Vol. } \\
(\mu L)\end{array}$ & $\begin{array}{l}\text { Conc. } \\
\text { (mg/mL) }\end{array}$ & $\begin{array}{l}\text { Conc. } \\
(\mathrm{mM})\end{array}$ \\
\hline FC-002 & 0.0095 & 230 & 950 & 10 & 43.48 & FC-517 & 0.0103 & 565 & 1030 & 10 & 17.70 \\
\hline FC-004 & 0.0098 & 161.5 & 980 & 10 & 61.92 & FC-518 & 0.0083 & 515 & 830 & 10 & 19.42 \\
\hline FC-006 & 0.0107 & 225.5 & 1070 & 10 & 44.35 & FC-520 & 0.0179 & 565 & 895 & 20 & 35.40 \\
\hline FC-007 & 0.0096 & 184.6 & 960 & 10 & 54.17 & FC-521 & 0.0045 & 429 & 450 & 10 & 23.31 \\
\hline FC-010 & 0.0093 & 163 & 930 & 10 & 61.35 & FC-523 & 0.0107 & 565 & 1070 & 10 & 17.70 \\
\hline FC-011 & 0.0088 & 199.5 & 880 & 10 & 50.13 & FC-524 & 0.0063 & $? ?$ & 630 & 10 & $? ?$ \\
\hline FC-017 & 0.0127 & 286 & 1270 & 10 & 34.97 & FC-526 & 0.0069 & $? ?$ & 690 & 10 & ?? \\
\hline FC-018 & 0.0124 & 262 & 1240 & 10 & 38.17 & FC-527 & 0.0185 & $? ?$ & 925 & 20 & $? ?$ \\
\hline FC-019 & 0.0092 & 301 & 920 & 10 & 33.22 & FC-531 & 0.0010 & 549 & 100 & 10 & 18.21 \\
\hline FC-023 & 0.0123 & 163 & 1230 & 10 & 61.35 & FC-532 & 0.0090 & 220 & 900 & 10 & 45.45 \\
\hline FC-024 & 0.0051 & 133 & 510 & 10 & 75.19 & FC-535 & 0.0103 & 534 & 1030 & 10 & 18.73 \\
\hline FC-025 & 0.0093 & 414 & 930 & 10 & 24.15 & FC-536 & 0.0060 & 630 & 600 & 10 & 15.87 \\
\hline FC-026 & 0.0111 & 194 & 1110 & 10 & 51.55 & FC-538 & 0.0311 & $?$ & 1555 & 20 & $? ?$ \\
\hline FC-027 & 0.0153 & 180 & 765 & 20 & 111.11 & FC-539 & 0.0128 & 264 & 1280 & 10 & 37.88 \\
\hline FC-028 & 0.0055 & 180 & 550 & 10 & 55.56 & FC-540 & 0.0027 & 270 & 270 & 10 & 37.04 \\
\hline FC-029 & 0.0104 & 194 & 1040 & 10 & 51.55 & FC-541 & 0.0052 & $? ?$ & 520 & 10 & $? ?$ \\
\hline FC-034 & 0.0097 & 232 & 970 & 10 & 43.10 & FC-542 & 0.0018 & 277 & 180 & 10 & 36.10 \\
\hline FC-035 & 0.0114 & 460 & 1140 & 10 & 21.74 & FC-543 & 0.0103 & 350 & 1030 & 10 & 28.57 \\
\hline FC-036 & 0.0116 & 221 & 1160 & 10 & 45.25 & FC-544 & 0.0067 & 310 & 670 & 10 & 32.26 \\
\hline FC-040 & 0.0104 & 194 & 1040 & 10 & 51.55 & FC-545 & 0.0123 & 278 & 1230 & 10 & 35.97 \\
\hline FC-041 & 0.0097 & 164 & 970 & 10 & 60.98 & FC-546 & 0.0134 & 370 & 1340 & 10 & 27.03 \\
\hline FC-042 & 0.0090 & 356 & 900 & 10 & 28.09 & FC-547 & 0.0035 & 264 & 350 & 10 & 37.88 \\
\hline FC-044 & 0.0084 & 215.5 & 840 & 10 & 46.40 & FC-548 & 0.0090 & 268 & 900 & 10 & 37.31 \\
\hline FC-045 & 0.0104 & 256.5 & 1040 & 10 & 38.99 & FC-549 & 0.0022 & $? ?$ & 220 & 10 & $? ?$ \\
\hline FC-046 & 0.0121 & 212 & 1210 & 10 & 47.17 & FC-550 & 0.0130 & 304 & 1300 & 10 & 32.89 \\
\hline FC-047 & 0.0119 & 194 & 1190 & 10 & 51.55 & FC-551 & 0.0084 & 248 & 840 & 10 & 40.32 \\
\hline FC-051 & 0.0096 & 370 & 960 & 10 & 27.03 & FC-552 & 0.0085 & 290 & 850 & 10 & 34.48 \\
\hline FC-054 & 0.0083 & 180 & 830 & 10 & 55.56 & FC-553 & 0.0100 & 268 & 1000 & 10 & 37.31 \\
\hline FC-056 & 0.0030 & 173 & 300 & 10 & 57.80 & FC-554 & 0.0106 & 350 & 1060 & 10 & 28.57 \\
\hline FC-057 & 0.0109 & 212 & 1090 & 10 & 47.17 & FC-555 & 0.0130 & 250 & 1300 & 10 & 40.00 \\
\hline FC-060 & 0.0070 & 203 & 700 & 10 & 49.26 & FC-556 & 0.0127 & 290 & 1270 & 10 & 34.48 \\
\hline FC-061 & 0.0137 & 161 & 1370 & 10 & 62.11 & FC-557 & 0.0152 & $? ?$ & 760 & 20 & $? ?$ \\
\hline FC-062 & 0.0120 & 308 & 1200 & 10 & 32.47 & FC-558 & 0.0065 & 306 & 650 & 10 & 32.68 \\
\hline FC-063 & 0.0104 & 323 & 1040 & 10 & 30.96 & FC-559 & 0.0010 & 288 & 100 & 10 & 34.72 \\
\hline FC-064 & 0.0115 & 473 & 1150 & 10 & 21.14 & FC-560 & 0.0164 & $? ?$ & 820 & 20 & $? ?$ \\
\hline FC-500 & 0.0165 & 357 & 825 & 20 & 56.02 & FC-561 & 0.0124 & 268 & 1240 & 10 & 37.31 \\
\hline FC-501 & 0.0095 & $?$ & 950 & 10 & $? ?$ & FC-562 & 0.0164 & 292 & 820 & 20 & 68.49 \\
\hline FC-504 & 0.0115 & $?$ & 1150 & 10 & $? ?$ & FC-563 & 0.0170 & 354 & 850 & 20 & 56.50 \\
\hline FC-506 & 0.0148 & $?$ & 1480 & 10 & $? ?$ & FC-564 & 0.0173 & 278 & 865 & 20 & 71.94 \\
\hline FC-507 & 0.0097 & 593 & 970 & 10 & 16.86 & FC-565 & 0.0050 & 402 & 500 & 10 & 24.88 \\
\hline FC-510 & 0.0140 & $?$ & 1400 & 10 & $? ?$ & FC-566 & 0.0138 & 268 & 1380 & 10 & 37.31 \\
\hline FC-512 & 0.0192 & 468 & 960 & 20 & 42.74 & FC-567 & 0.0108 & $? ?$ & 1080 & 10 & $? ?$ \\
\hline FC-513 & 0.0088 & $?$ & 880 & 10 & ?? & FC-568 & 0.0106 & 332 & 1060 & 10 & 30.12 \\
\hline FC-515 & 0.0147 & $?$ & 1470 & 10 & $? ?$ & FC-569 & 0.0013 & 266 & 130 & 10 & 37.59 \\
\hline
\end{tabular}




\begin{tabular}{|c|c|c|c|c|c|c|c|c|c|c|c|}
\hline Cat. \# & $\begin{array}{c}\text { Mass } \\
(\mathrm{g})\end{array}$ & $\begin{array}{l}\text { Mol. } \\
\text { mass }\end{array}$ & $\begin{array}{l}\text { Vol. } \\
(\mu \mathrm{L})\end{array}$ & $\begin{array}{l}\text { Conc. } \\
(\mathrm{mg} / \mathrm{mL})\end{array}$ & $\begin{array}{l}\text { Conc. } \\
(\mathbf{m M})\end{array}$ & Cat. \# & Mass (g) & $\begin{array}{l}\text { Mol. } \\
\text { mass }\end{array}$ & $\begin{array}{l}\text { Vol. } \\
(\mu \mathrm{L})\end{array}$ & $\begin{array}{l}\text { Conc. } \\
(\mathrm{mg} / \mathrm{mL})\end{array}$ & $\begin{array}{l}\text { Conc. } \\
\text { (mM) }\end{array}$ \\
\hline FC-570 & 0.0054 & 326 & 540 & 10 & 30.67 & FC-715 & 0.0317 & 190 & 1585 & 20 & 105.26 \\
\hline FC-571 & 0.0114 & 382 & 1140 & 10 & 26.18 & FC-717 & 0.0136 & 306 & 1360 & 10 & 32.68 \\
\hline FC-572 & 0.0084 & 350 & 840 & 10 & 28.57 & FC-732 & 0.0091 & 142 & 910 & 10 & 70.42 \\
\hline FC-573 & 0.0200 & 278 & 1000 & 20 & 71.94 & FC-733 & 0.0093 & 448.5 & 930 & 10 & 22.30 \\
\hline FC-574 & 0.0171 & 308 & 855 & 20 & 64.94 & FC-734 & 0.0028 & 415 & 280 & 10 & 24.10 \\
\hline FC-575 & 0.0159 & 282 & 795 & 20 & 70.92 & FC-735 & 0.0056 & 511 & 560 & 10 & 19.57 \\
\hline FC-576 & 0.0079 & 292 & 790 & 10 & 34.25 & FC-736 & 0.0038 & 486 & 380 & 10 & 20.58 \\
\hline FC-577 & 0.0142 & 306 & 1420 & 10 & 32.68 & FC-737 & 0.0076 & 413 & 760 & 10 & 24.21 \\
\hline FC-578 & 0.0049 & $? ?$ & 490 & 10 & $? ?$ & FC-738 & 0.0069 & 342 & 690 & 10 & 29.24 \\
\hline FC-579 & 0.0059 & $? ?$ & 590 & 10 & $? ?$ & FC-739 & 0.0094 & 550 & 940 & 10 & 18.18 \\
\hline FC-580 & 0.0174 & 264 & 870 & 20 & 75.76 & FC-740 & 0.0080 & 323 & 800 & 10 & 30.96 \\
\hline FC-581 & 0.0130 & 278 & 1300 & 10 & 35.97 & FC-741 & 0.0085 & 427 & 850 & 10 & 23.42 \\
\hline FC-582 & 0.0062 & 330 & 620 & 10 & 30.30 & FC-742 & 0.0107 & 387 & 1070 & 10 & 25.84 \\
\hline FC-583 & 0.0077 & 291 & 770 & 10 & 34.36 & FC-743 & 0.0136 & 429 & 1360 & 10 & 23.31 \\
\hline FC-584 & 0.0077 & 288 & 770 & 10 & 34.72 & FC-744 & 0.0136 & 443 & 1360 & 10 & 22.57 \\
\hline FC-585 & 0.0124 & 306 & 1240 & 10 & 32.68 & FC-745 & 0.0066 & 607 & 660 & 10 & 16.47 \\
\hline FC-586 & 0.0041 & 278 & 410 & 10 & 35.97 & FC-746 & 0.0099 & 698 & 990 & 10 & 14.33 \\
\hline FC-587 & 0.0133 & 291 & 1330 & 10 & 34.36 & FC-747 & 0.0102 & 661 & 1020 & 10 & 15.13 \\
\hline FC-588 & 0.0081 & 248 & 810 & 10 & 40.32 & FC-748 & 0.0046 & 557 & 460 & 10 & 17.95 \\
\hline FC-589 & 0.0174 & 322 & 870 & 20 & 62.11 & FC-749 & 0.0082 & 438 & 820 & 10 & 22.83 \\
\hline FC-590 & 0.0084 & 308 & 840 & 10 & 32.47 & FC-750 & 0.0157 & 502 & 785 & 20 & 39.84 \\
\hline FC-591 & 0.0148 & 306 & 1480 & 10 & 32.68 & FC-751 & 0.0107 & 376 & 1070 & 10 & 26.60 \\
\hline FC-592 & 0.0079 & 282 & 790 & 10 & 35.46 & FC-752 & 0.0072 & 562 & 720 & 10 & 17.79 \\
\hline FC-594 & 0.0137 & 275 & 1370 & 10 & 36.36 & FC-754 & 0.0050 & 550 & 500 & 10 & 18.18 \\
\hline FC-596 & 0.0135 & 308 & 1350 & 10 & 32.47 & FC-755 & 0.0144 & 376 & 1440 & 10 & 26.60 \\
\hline FC-597 & 0.0146 & 302 & 1460 & 10 & 33.11 & FC-756 & 0.0085 & 557 & 850 & 10 & 17.95 \\
\hline FC-598 & 0.0138 & 240 & 1380 & 10 & 41.67 & FC-776 & 0.0086 & 516 & 860 & 10 & 19.38 \\
\hline FC-599 & 0.0102 & 280 & 1020 & 10 & 35.71 & FC-777 & 0.0089 & 555 & 890 & 10 & 18.02 \\
\hline FC-665 & 0.0101 & 231 & 1010 & 10 & 43.29 & FC-779 & 0.0136 & 477 & 1360 & 10 & 20.96 \\
\hline FC-674 & 0.0091 & 103 & 910 & 10 & 97.09 & FC-785 & 0.0028 & 462 & 280 & 10 & 21.65 \\
\hline FC-676 & 0.0053 & 159 & 530 & 10 & 62.89 & FC-787 & 0.0069 & $?$ & 690 & 10 & ?? \\
\hline FC-677 & 0.0071 & 219 & 710 & 10 & 45.66 & FC-788 & 0.0090 & 279 & 900 & 10 & 35.84 \\
\hline FC-678 & 0.0055 & 300 & 550 & 10 & 33.33 & FC-792 & 0.0136 & 434 & 1360 & 10 & 23.04 \\
\hline FC-679 & 0.0081 & 251 & 810 & 10 & 39.84 & FC-797 & 0.0216 & 429 & 1080 & 20 & 46.62 \\
\hline FC-680 & 0.0115 & 89 & 1150 & 10 & 112.36 & FC-805 & 0.0066 & 322 & 660 & 10 & 31.06 \\
\hline FC-682 & 0.0051 & 192 & 510 & 10 & 52.08 & FC-808 & 0.0143 & $?$ & 1430 & 10 & ?? \\
\hline FC-683 & 0.0143 & 295 & 1430 & 10 & 33.90 & FC-810 & 0.0010 & 161 & 100 & 10 & 62.11 \\
\hline FC-684 & 0.0010 & $? ?$ & 100 & 10 & ?? & FC-811 & 0.0041 & 295 & 410 & 10 & 33.90 \\
\hline FC-685 & 0.0026 & $?$ & 260 & 10 & ?? & FC-812 & 0.0020 & 325 & 200 & 10 & 30.77 \\
\hline FC-694 & 0.0109 & 189 & 1090 & 10 & 52.91 & FC-815 & 0.0224 & 190 & 1120 & 20 & 105.26 \\
\hline FC-697 & 0.0134 & 363 & 1340 & 10 & 27.55 & FC-816 & 0.0182 & 121 & 910 & 20 & 165.29 \\
\hline FC-698 & 0.0124 & 226 & 1240 & 10 & 44.25 & FC-817 & 0.0055 & 121 & 550 & 10 & 82.64 \\
\hline FC-699 & 0.0079 & $?$ & 790 & 10 & ?? & FC-822 & 0.0027 & 147 & 270 & 10 & 68.03 \\
\hline FC-700 & 0.0041 & $?$ & 410 & 10 & ?? & FC-823 & 0.0046 & 121 & 460 & 10 & 82.64 \\
\hline FC-701 & 0.0213 & $?$ & 1065 & 20 & ?? & FC-825 & 0.0057 & 161 & 570 & 10 & 62.11 \\
\hline FC-708 & 0.0173 & 554 & 865 & 20 & 36.10 & FC-826 & 0.0171 & 187 & 855 & 20 & 106.95 \\
\hline FC-709 & 0.0132 & 540 & 1320 & 10 & 18.52 & FC-827 & 0.0063 & 147 & 630 & 10 & 68.03 \\
\hline FC-710 & 0.0188 & 572 & 940 & 20 & 34.97 & FC-828 & 0.0058 & 269 & 580 & 10 & 37.17 \\
\hline FC-711 & 0.0193 & 572 & 965 & 20 & 34.97 & FC-830 & 0.0037 & 265 & 370 & 10 & 37.74 \\
\hline FC-714 & 0.0131 & 454 & 1310 & 10 & 22.03 & FC-831 & 0.0215 & 283 & 1075 & 20 & 70.67 \\
\hline
\end{tabular}




\begin{tabular}{|c|c|c|c|c|c|c|c|c|c|c|c|}
\hline Cat. \# & $\begin{array}{c}\text { Mass } \\
\text { (g) }\end{array}$ & $\begin{array}{l}\text { Mol. } \\
\text { mass }\end{array}$ & $\begin{array}{l}\text { Vol. } \\
(\mu \mathrm{L})\end{array}$ & $\begin{array}{l}\text { Conc. } \\
\text { (mg/mL) }\end{array}$ & $\begin{array}{l}\text { Conc. } \\
(\mathrm{mM})\end{array}$ & Cat. \# & Mass (g) & $\begin{array}{l}\text { Mol. } \\
\text { mass }\end{array}$ & Vol. $(\mu \mathrm{L})$ & $\begin{array}{l}\text { Conc. } \\
(\mathrm{mg} / \mathrm{mL})\end{array}$ & $\begin{array}{l}\text { Conc. } \\
(\mathrm{mM})\end{array}$ \\
\hline FC-832 & 0.0070 & 352 & 700 & 10 & 28.41 & FC-910 & 0.0155 & 152 & 775 & 20 & 131.58 \\
\hline FC-834 & 0.0115 & 151 & 1150 & 10 & 66.23 & FC-911 & 0.0033 & & 330 & 10 & ?? \\
\hline FC-836 & 0.0093 & 254 & 930 & 10 & 39.37 & FC-912 & 0.0113 & 437 & 1130 & 10 & 22.88 \\
\hline FC-837 & 0.0025 & 282 & 250 & 10 & 35.46 & FC-913 & 0.0145 & 152 & 1450 & 10 & 65.79 \\
\hline FC-838 & 0.0039 & 147 & 390 & 10 & 68.03 & FC-914 & 0.0125 & 290 & 1250 & 10 & 34.48 \\
\hline FC-845 & 0.0253 & 337 & 1265 & 20 & 59.35 & FC-915 & 0.0150 & 429 & 1500 & 10 & 23.31 \\
\hline FC-853 & 0.0058 & 278 & 580 & 10 & 35.97 & FC-916 & 0.0096 & 436 & 960 & 10 & 22.94 \\
\hline FC-854 & 0.0177 & 194 & 885 & 20 & 103.09 & FC-917 & 0.0094 & 152 & 940 & 10 & 65.79 \\
\hline FC-856 & 0.0010 & 174.5 & 100 & 10 & 57.31 & FC-918 & 0.0069 & 289 & 690 & 10 & 34.60 \\
\hline FC-858 & 0.0010 & 202 & 100 & 10 & 49.50 & FC-919 & 0.0066 & 330 & 660 & 10 & 30.30 \\
\hline FC-859 & 0.0146 & 250.5 & 1460 & 10 & 39.92 & FC-920 & 0.0117 & 374 & 1170 & 10 & 26.74 \\
\hline FC-864 & 0.0272 & 344 & 1360 & 20 & 58.14 & FC-921 & 0.0093 & 374 & 930 & 10 & 26.74 \\
\hline FC-866 & 0.0010 & 288 & 100 & 10 & 34.72 & FC-922 & 0.0134 & 152 & 1340 & 10 & 65.79 \\
\hline FC-867 & 0.0234 & 266 & 1170 & 20 & 75.19 & FC-923 & 0.0056 & 170.5 & 560 & 10 & 58.65 \\
\hline FC-868 & 0.0047 & 288 & 470 & 10 & 34.72 & FC-925 & 0.0099 & 169 & 990 & 10 & 59.17 \\
\hline FC-869 & 0.0011 & 326 & 110 & 10 & 30.67 & FC-926 & 0.0074 & 494 & 740 & 10 & 20.24 \\
\hline FC-870 & 0.0014 & 238 & 140 & 10 & 42.02 & FC-927 & 0.0023 & 517 & 230 & 10 & 19.34 \\
\hline FC-871 & 0.0010 & 284 & 100 & 10 & 35.21 & FC-928 & 0.0117 & 152 & 1170 & 10 & 65.79 \\
\hline FC-873 & 0.0070 & 380 & 700 & 10 & 26.32 & FC-929 & 0.0090 & 181 & 900 & 10 & 55.25 \\
\hline FC-874 & 0.0020 & 250 & 200 & 10 & 40.00 & FC-930 & 0.0208 & 315 & 1040 & 20 & 63.49 \\
\hline FC-875 & 0.0075 & 106.5 & 750 & 10 & 93.90 & FC-931 & 0.0080 & 315 & 800 & 10 & 31.75 \\
\hline FC-876 & 0.0097 & 208 & 970 & 10 & 48.08 & FC-932 & 0.0147 & 509 & 1470 & 10 & 19.65 \\
\hline FC-878 & 0.0125 & 324.5 & 1250 & 10 & 30.82 & FC-933 & 0.0089 & 300 & 890 & 10 & 33.33 \\
\hline FC-879 & 0.0043 & 156.5 & 430 & 10 & 63.90 & FC-935 & 0.0029 & 495 & 290 & 10 & 20.20 \\
\hline FC-880 & 0.0075 & 300 & 750 & 10 & 33.33 & FC-936 & 0.0100 & 300 & 1000 & 10 & 33.33 \\
\hline FC-881 & 0.0083 & 312 & 830 & 10 & 32.05 & FC-937 & 0.0022 & 517 & 220 & 10 & 19.34 \\
\hline FC-882 & 0.0164 & 258 & 820 & 20 & 77.52 & FC-938 & 0.0124 & 227 & 1240 & 10 & 44.05 \\
\hline FC-883 & 0.0030 & 386.5 & 300 & 10 & 25.87 & FC-939 & 0.0051 & 227 & 510 & 10 & 44.05 \\
\hline FC-884 & 0.0137 & 374.5 & 1370 & 10 & 26.70 & FC-940 & 0.0136 & 507 & 1360 & 10 & 19.72 \\
\hline FC-885 & 0.0071 & 244.5 & 710 & 10 & 40.90 & FC-941 & 0.0030 & 641 & 300 & 10 & 15.60 \\
\hline FC-886 & 0.0077 & 244.5 & 770 & 10 & 40.90 & FC-942 & 0.0100 & 626 & 1000 & 10 & 15.97 \\
\hline FC-887 & 0.0148 & 404.5 & 1480 & 10 & 24.72 & FC-944 & 0.0061 & 506 & 610 & 10 & 19.76 \\
\hline FC-888 & 0.0223 & 177 & 1115 & 20 & 112.99 & FC-945 & 0.0082 & 173 & 820 & 10 & 57.80 \\
\hline
\end{tabular}

Table 7.1 Molecular data on compound library from IRL. Library consists of synthetic compounds and synthetic derivatives of diterpine natural products. They are dissolved in DMSO, and stored at $-20^{\circ} \mathrm{C}$ at the Chemical Genetics Laboratory, Victoria University of Wellington. 
7.1.2 Appendix 1 - Plate layout data for dose response screen

\begin{tabular}{|c|c|c|c|c|c|c|c|c|}
\hline Compound & Row A & Row B & Row C & Row D & Row E & Row F & Row G & Row H \\
\hline FC-018 & 381.68 & 120.78 & 38.22 & 12.10 & 3.83 & 1.21 & 0.38 & 0.12 \\
\hline FC-526 & ?? & ?? & ?? & ?? & ?? & ?? & ?? & ?? \\
\hline FC-561 & 373.13 & 118.08 & 37.37 & 11.83 & 3.74 & 1.18 & 0.37 & 0.12 \\
\hline FC-566 & 373.13 & 118.08 & 37.37 & 11.83 & 3.74 & 1.18 & 0.37 & 0.12 \\
\hline FC-567 & ?? & ?? & ?? & ?? & ?? & ?? & ?? & ?? \\
\hline FC-570 & 306.75 & 97.07 & 30.72 & 9.72 & 3.08 & 0.97 & 0.31 & 0.10 \\
\hline FC-571 & 261.78 & 82.84 & 26.22 & 8.30 & 2.63 & 0.83 & 0.26 & 0.08 \\
\hline FC-574 & 649.35 & 205.49 & 65.03 & 20.58 & 6.51 & 2.06 & 0.65 & 0.21 \\
\hline FC-575 & 354.61 & 112.22 & 35.51 & 11.24 & 3.56 & 1.13 & 0.36 & 0.11 \\
\hline FC-578 & ?? & ?? & ?? & ?? & ?? & ?? & $? ?$ & ?? \\
\hline FC-580 & 757.58 & 239.74 & 75.87 & 24.01 & 7.60 & 2.40 & 0.76 & 0.24 \\
\hline FC-582 & 303.03 & 95.90 & 30.35 & 9.60 & 3.04 & 0.96 & 0.30 & 0.10 \\
\hline FC-588 & 403.23 & 127.60 & 40.38 & 12.78 & 4.04 & 1.28 & 0.40 & 0.13 \\
\hline FC-589 & 621.12 & 196.56 & 62.20 & 19.68 & 6.23 & 1.97 & 0.62 & 0.20 \\
\hline FC-592 & 354.61 & 112.22 & 35.51 & 11.24 & 3.56 & 1.13 & 0.36 & 0.11 \\
\hline FC-596 & 324.68 & 102.75 & 32.51 & 10.29 & 3.26 & 1.03 & 0.33 & 0.10 \\
\hline FC-599 & 357.14 & 113.02 & 35.77 & 11.32 & 3.58 & 1.13 & 0.36 & 0.11 \\
\hline FC-697 & 275.48 & 87.18 & 27.59 & 8.73 & 2.76 & 0.87 & 0.28 & 0.09 \\
\hline FC-732 & 704.23 & 222.86 & 70.52 & 22.32 & 7.06 & 2.23 & 0.71 & 0.22 \\
\hline FC-734 & 240.96 & 76.25 & 24.13 & 7.64 & 2.42 & 0.76 & 0.24 & 0.08 \\
\hline FC-856 & 573.07 & 181.35 & 57.39 & 18.16 & 5.75 & 1.82 & 0.58 & 0.18 \\
\hline FC-888 & 381.68 & 120.78 & 38.22 & 12.10 & 3.83 & 1.21 & 0.38 & 0.12 \\
\hline CHX & 10.00 & 3.16 & 1.00 & 0.32 & 0.10 & 0.03 & 0.01 & 0.00 \\
\hline
\end{tabular}

Table 7.2 Plate layout for original dose response experiment assessing potency of hits from the halo assay. All concentrations are given in $\mu \mathrm{M}$. Molecular data was unknown for several compounds, and thus molar concentrations could not be calculated. Cycloheximide (CHX) served as a positive control, as an inhibitory compound of known potency. 
A multi condition experiment was conducted to identify the cause of the 'bubbles' seen in the early

halo assays.

A

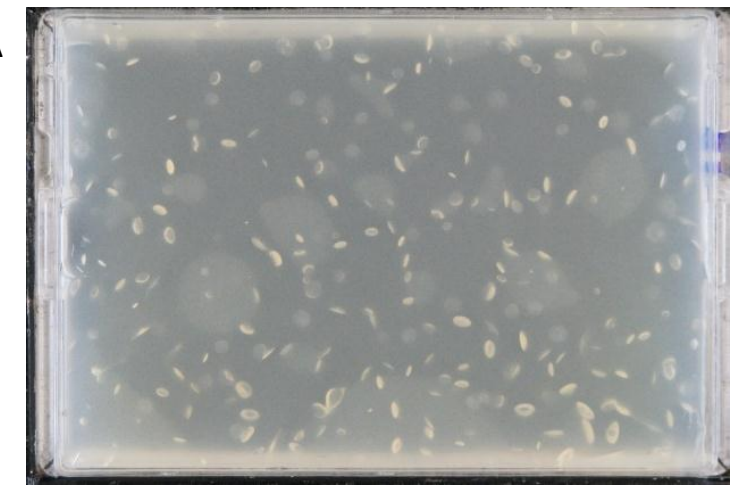

C

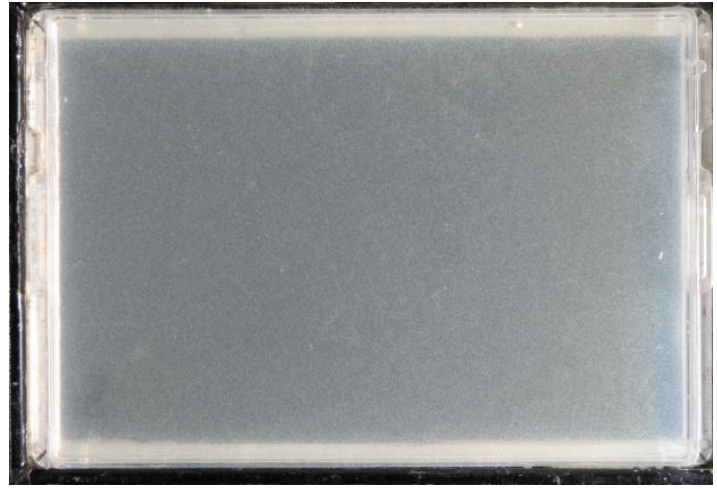

$\mathrm{E}$

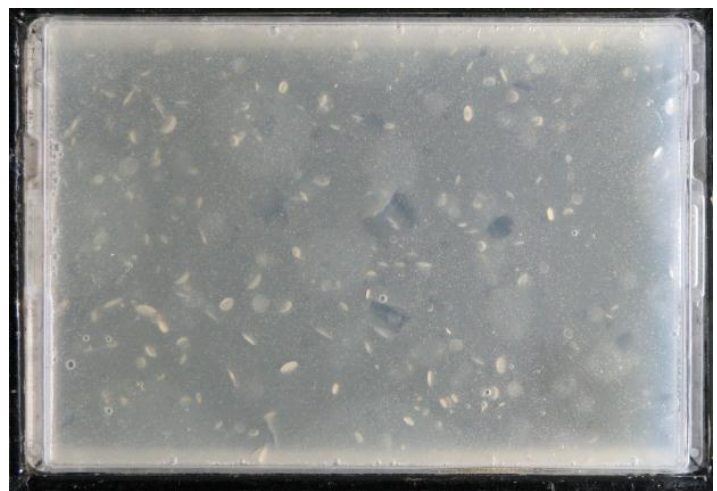

B

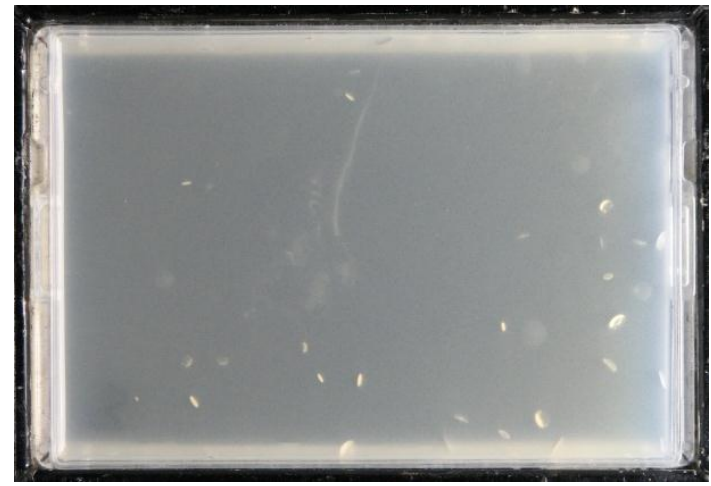

D

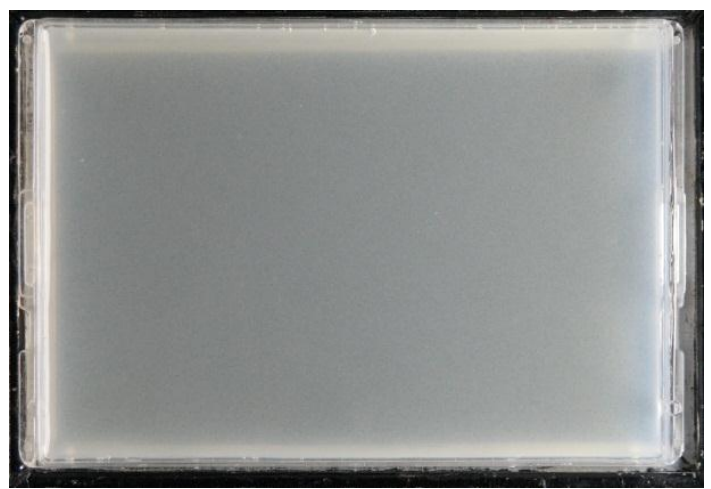

$\mathrm{F}$

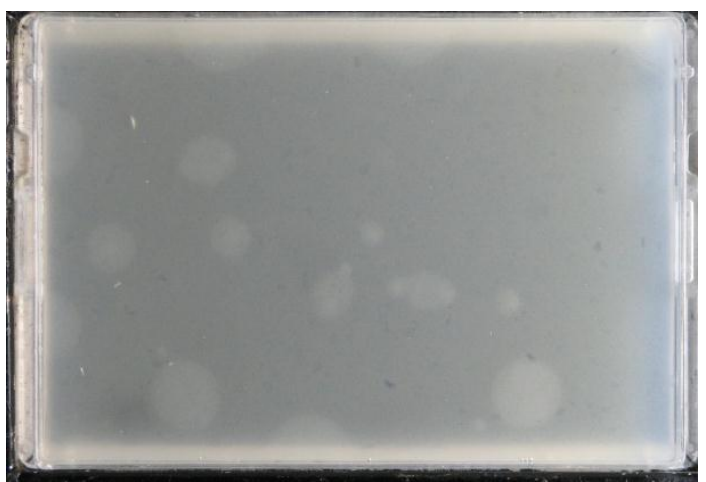

Figure 7.1 Conditional test based on the halo assay. Tests were conducted to determine the cause of bubbling seen in the HEPES buffered halo assay. A: original assay SC Agar, $\sim 2 \times 10^{5}$ cells, $2 \%$ glucose, $25 \mathrm{mM}$ HEPES buffer. B: as A with reduced glucose $(0.5 \%)$ C: as A with reduced cell concentration $\left(\sim 2 \times 10^{4}\right)$. D: as A with no HEPES buffer. E: as A with HEPES replaced with $100 \mathrm{mM} \mathrm{NaHCO}_{3}$. F: as A with HEPES replaced with $25 \mathrm{mM}$ MOPS. $\mathrm{B}$ and $\mathrm{C}$ indicate that bubble formation is mediated by cellular metabolism: less cells or cells growing slower due to reduced nutrients produce fewer bubbles. Plate D suggests a buffer mediated effect. Of the three buffers, (A, E, F), MOPS produces plates with the fewest bubbles. 


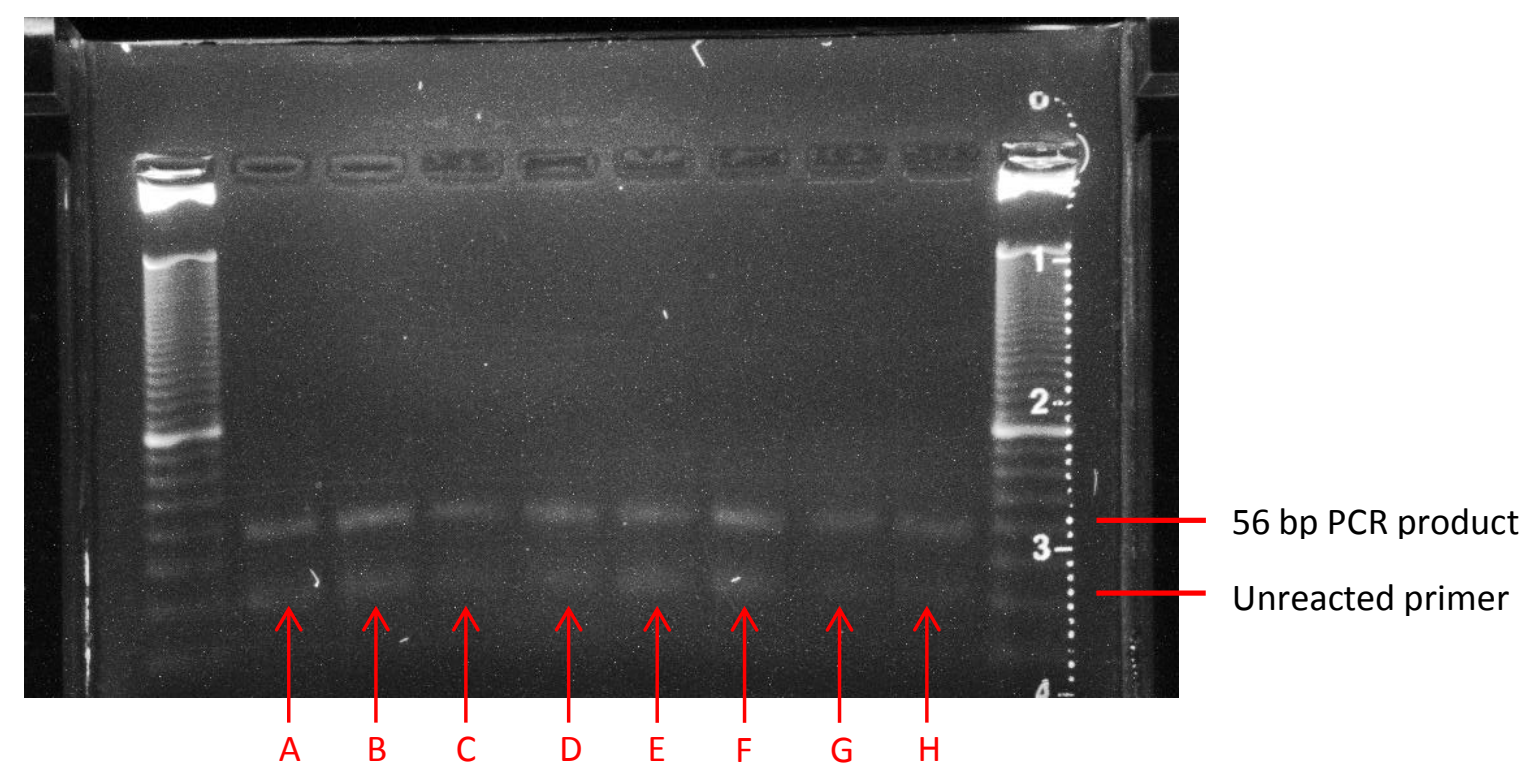

Figure 7.2 Confirmation of PCR product for HOP screen. Cy labelled 56 bp PCR products were confirmed by $4 \%$ MetaPhor agarose gel electrophoresis, validating HOP screen data. FC-592 treated cells (and controls): A: Cy5 labelled UP tags. B: Cy5 labelled DN tags. C: Cy3 labelled UP tags. D: Cy3 labelled DN tags. FC-888 treated cells (and controls): E: Cy5 labelled UP tags. F: Cy5 labelled DN tags. G: Cy3 labelled UP tags. H: Cy3 labelled DN tags. A faint band representing unreacted primer was also seen.

\subsection{Appendix 4 - tetrad analysis of FC-592 resistant mutants}

Mutants were selected for complementation testing based on tetrad analysis of cells. YCG434 was crossed with a Mat A his3 $3:: K a n R ;$ pdr1 $\triangle:: N A T ;$ pdr3 $\Delta::$ URA3 mutant obtained from Namal Coorey. This diploid was sporulated and dissected as described in Chapter 4. Meiotic progeny were screened for mating type, FC-592 resistance, loss of CenLeu plasmid so that this selection could be reused, and G418 resistance.

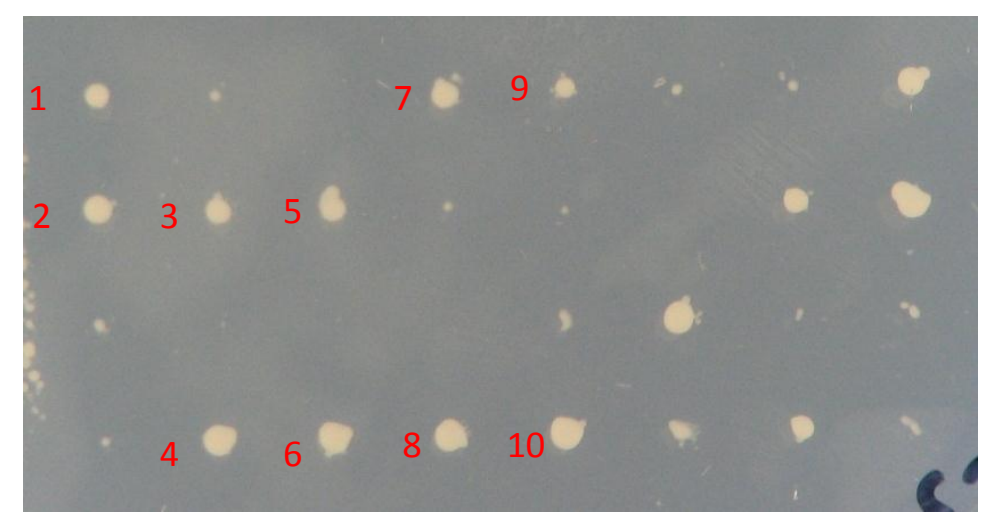

Figure 7.3 Mat A selection of YCG434 meiotic progeny. Tetrad plates were replica plated onto Mat A selective media (SD -His, -Arg, -Lys, +Can, +Thia). Mat $A$ segregants were labelled and transferred to additional selections. 


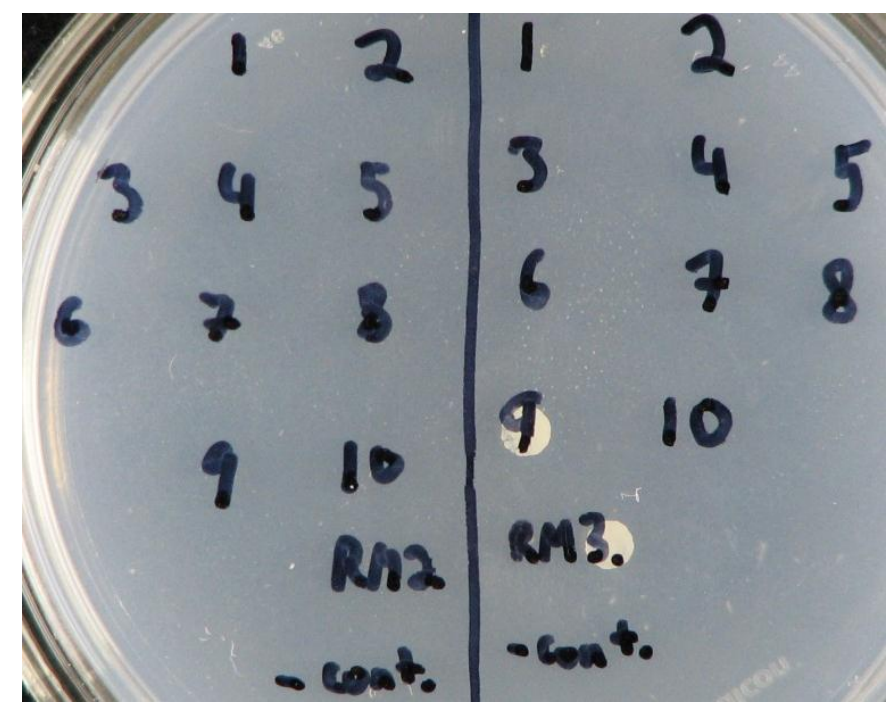

Figure 7.4 Selection on 150 MM FC-592 media. Only tetrad \# 9 still carried the FC-592 resistance mutation. The reason for this is unknown, the mutation had previously been characterised as single locus mediated via tetrad analysis (discussed in Chapter 4).
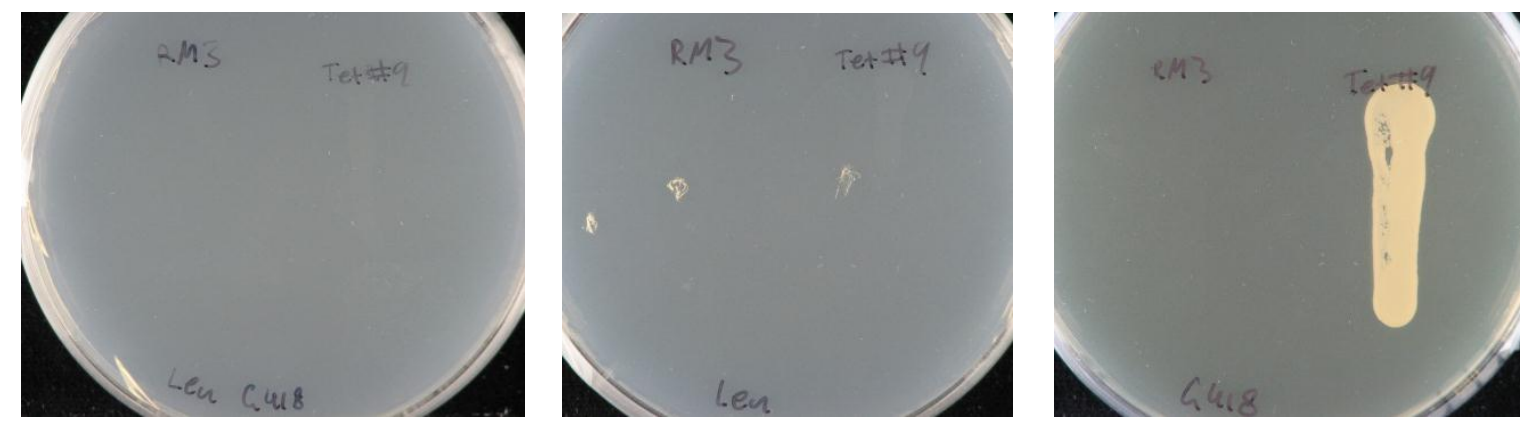

Figure 7.5 Phenotyping of Tetrad \#9. Tetrad \#9 displayed no growth on SD-Leu +G418 media (I) and SD-Leu (m). It did however grow on $\mathrm{SC}+\mathrm{G} 418$ media ( $\mathrm{r}$ ). This shows that this strain has lost its CenLeu plasmid, which mediates resistance to leucine auxotrophy. However, it grew in the presence of g418, showing that it retained the KanR resistance cassette. YCG434 (here labelled RM3), was used as a control.

Data from this analysis identified Tetrad \#9 as Mat A, CenLeu-, FC-592R+, and KanR+. This made it suitable for mating with a CenLeu transformed YCG433 strain for complementation, as it enabled diploid selection. 


\section{References}

Abumrad N, Coburn C \& Ibrahimi A (1999). Membrane proteins implicated in long-chain fatty acid uptake by mammalian cells: CD36, FATP and FABPm. Biochimica et Biophysica ActaMolecular and Cell Biology of Lipids 1441(1): Oct. 1999. pp. 4-13. DOI: 10.1016/S13881981(99)00137-7

Ahmed KA \& Woods RA (1967). A genetic analysis of resistance to nystatin in Saccharomyces cerevisiae. Genetical Research 9(2): pp. 179-180.

Akache B, MacPherson S, Sylvain MA, et al. (2004). Complex interplay among regulators of drug resistance genes in Saccharomyces cerevisiae. J Biol Chem 279(27): Jul. 2004. pp. 2785527860. DOI: $10.1074 / j b c . M 403487200$

Alamgir M, Erukova V, Jessulat M, et al. (2010). Chemical-genetic profile analysis of five inhibitory compounds in yeast. BMC Chemical Biology 2010 10(6): DOI: 10.1186/14726769-10-6

Anastasia SD, Nguyen DL,; Vu T, et al. (2012). A link between mitotic entry and membrane growth suggests a novel model for cell size control. Journal of Cell Biology 197(1): Apr. 2012. pp. 89-104. DOI: $10.1083 / j c b .201108108$

Anderson KC (2005). Lenalidomide and thalidomide: mechanisms of action-similarities and differences. Seminars in Hematology 42(4 Suppl 4): Oct. 2005. pp. S3-S8. DOI:10.1053/j.seminhematol.2005.10.001.

Anson BD, Ma J \& He JQ (2009). Identifying cardiotoxic compounds. Genetic Engineering \& Biotechnology News 29(9): pp. 34-35. ISSN 1935-472X. OCLC 77706455.

Ariad Pharmaceuticals, Inc., Massachusetts Institute of Technology, The Whitehead Institute for Biomedical Research, and The President and Fellows of Harvard College, V. Eli Lilly and Company. 2008-1248 (United States Court of Appeals for the Federal Circuit 2009-04-03)

Arias CA \& Murray BE (2009). Antibiotic-resistant bugs in the 21st century - a clinical superchallenge. New England Journal of Medicine 360(5): Jan. 2009. pp. 439-443. DOI:10.1056/NEJMp0804651.

Avrahami-Moyal L, Braun S \& Engelberg D (2012). Overexpression of PDE2 or SSD1-V in Saccharomyces cerevisiae W303-1A strain renders it ethanol-tolerant. FEMS Yeast Research 12(4): Jun. 2012. pp. 447-455. DOI: 10.1111/j.1567-1364.2012.00795.x

Awad S, Panchy N, Ng SK, et al. (2012). Inferring the regulatory interaction models of transcription factors in transcriptional regulatory networks. Journal of Bioinformatics and Computational Biology 10(5): Jun. 2012. pp. 1250012. PMID: 22849367

Backer J \& Foury F (1985). Repair properties in yeast mitochondrial-DNA mutators. Current Genetics 10(1): pp. 7-13. DOI: 10.1007/BF00418487

Badr CE, Hewett JW, Breakefield XO, et al. (2007). A highly sensitive assay for monitoring the secretory pathway and ER stress. PLoS ONE 2(6): Jun. 2007. Art. e571. DOI: 10.1371/journal.pone.0000571 
Balani SK, Miwa GT, Gan LS, et al. (2005). Strategy of utilizing in vitro and in vivo ADME tools for lead optimization and drug candidate selection. Curr. Top. Med. Chem. 5(11): pp. 10331038. DOI: $10.2174 / 156802605774297038$

Ball LG, Zhang K, Cobb JA, et al. (2009). The yeast Shu complex couples error-free post-replication repair to homologous recombination. Molecular Microbiology 73(1): Jul. 2009. pp. 89102. DOI: $10.1111 / j .1365-2958.2009 .06748 . x$

Ball SG, Wickner RB, Cottarel G, et al. (1986). Molecular-cloning and characterization of ARO7OSM2, a single yeast gene necessary for chorismate mutase activity and growth in hypertonic medium. Molecular \& General Genetics 205(2): pp. 326-330. DOI: 10.1007/BF00430446

Balzi E \& Goffeau A (1991). Multiple or pleiotropic drug-resistance in yeast. Biochimica Et Biophysica Acta 1073(2): Mar. 1991. pp. 241-252. DOI: 10.1016/0304-4165(91)90128-4

Balzi E \& Goffeau A (1995). Yeast multidrug resistance: The PDR network. Journal of Bioenergetics and Biomembranes 27(1): Feb. 1995. pp. 71-76. DOI: 10.1007/BF02110333

Balzi E, Chen W, Ulaszewski S, et al. (1987). The multidrug resistance gene PDR1 from Saccharomyces cerevisiae. J Biol Chem. 262(35): Dec. 1987. pp. 16871-9. PMID: 3316228

Barrett D (2002). From natural products to clinically useful antifungals. Biochimica et Biophysica Acta (BBA) - Molecular Basis of Disease 1587(2-3): Jul. 2002. pp. 224-233. DOI: 10.1016/S0925-4439(02)00085-6.

Barton JH \& Emanuel EJ (2005). The patents-based pharmaceutical development process: rationale, problems and potential reforms. Journal of the American Medical Association 294(16): Oct. 2005. pp. 2075-82. DOI: 10.1001/jama.294.16.2075.

Baryshnikova A, Costanzo M, Dixon S, et al. (2010). Synthetic Genetic Array (SGA) analysis in Saccharomyces cerevisiae and Schizosaccharomyces pombe. Methods In Enzymology 470: pp. 145-179 DOI: 10.1016/S0076-6879(10)70007-0

Beninsone P, Miret JJ \& Hirschberg CB (1994). The Golgi guanosine diphosphatase is required for transport of GDP-mannose into the lumen of Saccharomyces cerevisiae Golgi vesicles. J. Biol. Chem. 269(1): pp. 207-211. PMID: 7506254

Bergman A \& Siegal ML (2003). Evolutionary capacitance as a general feature of complex gene networks. Nature 424(6948): Jul. 2003. pp. 549-552. DOI: 10.1038/nature01834

Bernales S, Papa FR \& Walter PB (2006). Intracellular signaling by the unfolded protein response. Annual Review of Cell and Developmental Biology 22: pp. 487-508. DOI: 10.1146/annurev.cellbio.21.122303.120200

Bharadwaj R \& Yu HT (2004). The spindle checkpoint, aneuploidy, and cancer. Oncogene 23(11): Mar. 2004. pp. 2016-2027. DOI: 10.1038/sj.onc.1207374

Bible KC \& Kaufmann SH (1997). Cytotoxic synergy between flavopiridol (NSC 649890, L86-8275) and various antineoplastic agents: The importance of sequence of administration. Cancer Research 57(16): Aug. 1997. pp. 3375-3380. PMID: 9269999 
Bircham PW, Maass DR, Roberts CA, et al. (2011). Secretory pathway genes assessed by highthroughput microscopy and synthetic genetic array analysis. Molecular Biosystems 7(9): pp. 2589-2598. DOI: 10.1039/c1mb05175j

Blackman RK, Cheung-Ong K, Gebbia M, et al. (2012). Mitochondrial electron transport is the cellular target of the oncology drug elesclomol. PLoS One 7(1): Jan. 2012. Art. e29798. DOI: 10.1371/journal.pone.0029798

Blanchard JS (1996). Molecular mechanisms of drug resistance in Mycobacterium tuberculosis. Annual Review of Biochemistry 65: pp. 215-239. DOI: 10.1146/annurev.biochem.65.1.215

Blunt JW, Copp BR, Hu WP, et al. (2008). Marine natural products. Natural Product Reports 25(1): Feb. 2008. pp. 35-94. DOI: 10.1039/b701534h

Bodor N \& Buchwald P (2000). Soft drug design: General principles and recent applications. Medicinal Research Reviews 20(1): Jan. 2000. pp. 58-101. DOI: 10.1002/(SICI)10981128(200001)20:1<58::AID-MED3>3.3.CO;2-O

Bolard J (1986). How do the polyene macrolide antibiotics affect the cellular membrane properties? Biochimica et Biophysica Acta 864(3-4): Dec. 1986. 257-304. DOI: 10.1016/0304-4157(86)90002-X

Boone C, Bussey H, \& Andrews BJ (2007). Exploring genetic interactions and networks with yeast. Nature Reviews Genetics 8(6): Jun 2007. pp. 437-449. DOI: 10.1038/nrg2085

Bose DS, Thompson AS, Ching JS, et al. (1992). Rational design of a highly efficient irreversible dna interstrand cross-linking agent based on the pyrrolobenzodiazepine ring-system. Journal of the American Chemical Society 114(12): Jun. 1992. pp. 4939-4941. DOI: 10.1021/ja00038a089

Bray PG, Barrett MP, Ward SA, et al. (2003). Pentamidine uptake and resistance in pathogenic protozoa: past, present and future. Trends in Parasitology 19(5): May 2003. pp. 232-239. DOI: 10.1016/S1471-4922(03)00069-2

Brenner S, Johnson M, Bridgham J, et al. (2000). Gene expression analysis by massively parallel signature sequencing (MPSS) on microbead arrays. Nature Biotechnology 18(6): Jun. 2000. pp. 630-634. DOI: 10.1038/76469

Broach JR, Strathern J \& Hicks JB (1979). Transformation in yeast - development of a hybrid cloning vector and isolation of the CAN1-gene. Gene 8(1): pp. 121-133. DOI: 10.1016/0378-1119(79)90012-X

Broekaert WF, Terras FRG, Cammue BPA \& Vanderleyden J (1990). An automated quantitative assay for fungal growth inhibition. FEMS Microbiology Letters 69(1-2): May 1990. pp. 5559. DOI: 10.1016/0378-1097(90)90412-J

Brooker RJ (2011). Genetics: analysis and principles (4th ed.). McGraw-Hill Science. New York, NY. ISBN 978-0-07-352528-0.

Brown W \& Poon T (2005). Organic chemistry, $3^{\text {rd }}$ ed. John Wiley \& Sons. New York, NY. ISBN-10: 0471444510 
Buey RM, Diaz JF, Andreu JM, et al. (2004. Interaction of epothilone analogs with the paclitaxel binding site: Relationship between binding affinity, microtubule stabilization, and cytotoxicity. Chemistry \& Biology 11(2): Feb. 2004. pp. 225-236. DOI: 10.1016/j.chembiol.2004.01.014

Burke DJ, Amberg DC \& Strathern JN (2005). Methods in yeast genetics: A Cold Spring Harbor laboratory course manual, 2005 Ed. Cold Spring Harbor Laboratory Press, Cold Spring Harbor, NY. ISBN-10: 0879697288

Burke M \& Schreiber SL (2004). A planning strategy for diversity-oriented synthesis. Angewandte Chemie International Edition 43(1): pp. 46-58. DOI:10.1002/anie.200300626

Burkholz H (1997). Giving thalidomide a second chance. FDA Consumer 31: Jun. 1997. US FDA.

Butler MS (2004). The role of natural product chemistry in drug discovery. J. Nat. Prod. 67(12): Dec. 2004. pp. 2141-2153. DOI: 10.1021/np040106y

Capson TL (2009). Biodiscovery research in Panama: Linking science, technology, human health, and conservation in the host-country context. In Contracting for ABS: The Legal and Scientific Implications of Bioprospecting Contracts. pp. 269-305. IUCN Environmental Policy and Law. Bonn, Germany. ISBN: 978-2-8317-0982-6

Carlson B, Lahusen T, Singh S, et al. (1999). Down-regulation of cyclin D1 by transcriptional repression in MCF-7 human breast carcinoma cells induced by flavopiridol. Cancer Research 59(18): Sep. 1999. pp. 4634-4641. PMID: 10493518

Carlson M, Osmond BC \& Botstein D (1981). Mutants of yeast defective in sucrose utilization. Gentetics 98(1): pp. 25-40. PMID: 7040163

Carragher NO, Unciti-Broceta A \& Cameron DA (2012). Advancing cancer drug discovery towards more agile development of targeted combination therapies. Future Medicinal Chemistry 4(1): Jan. 2012. pp. 87-105, DOI: 10.4155/fmc.11.169

Casagrande R, Stern P, Diehn M, et al. (2000) Degradation of proteins from the ER of S. cerevisiae requires an intact unfolded protein response pathway. Molecular Cell 5(4): Apr. 2000. pp. 729-735. DOI: 10.1016/S1097-2765(00)80251-8

Chanda SK \& Caldwell JS (2003). Fulfilling the promise: drug discovery in the post-genomic era. Drug Discovery Today 8(4): Feb. 2003. pp. 168-174. DOI: 10.1016/S1359-6446(02)02595-3

Chapes SK, Killion JW \& Morrison, DC (1988). Tumor-cell killing and cytostasis by C3H-HEJ macrophages activated in vitro by lipid A-associated protein and interferon-gamma. Journal of Leukocyte Biology 43(3): Mar. 1988. pp. 232-237. PMID: 3125294

Chen RH, Brady DM, Smith D, et al. (1999). The spindle checkpoint of budding yeast depends on a tight complex between the Mad1 and Mad2 proteins. Molecular Biology of the Cell 10(8): Aug. 1999. pp. 2607-2618.

Clapper ML \& Tew KD (1989). Alkylating agent resistance. Cancer Treatment and Research 48: pp. 125-150. PMID: 2577134

Clardy J \& Walsh C (2004). Lessons from natural molecules. Nature 432(7019): Dec. 2004. pp. 829-837. DOI: 10.1038/nature03194 
Colantuoni C, Henry G, Zeger S, et al. (2002). SNOMAD (Standardization and NOrmalization of MicroArray Data): web-accessible gene expression data analysis. Bioinformatics 18(11): Nov. 2002. pp. 1540-1541. DOI: 10.1093/bioinformatics/18.11.1540

Collinson LP \& Dawes IW (1992). Inducibility of the response of yeast cells to peroxide stress. Microbiology 138(2): Feb. 1992. pp. 329-335. DOI: 10.1099/00221287-138-2-329

Convention on Biological Diversity (2010). "Text of the Nagoya Protocol". CBD. Int. Convention on Biological Diversity. Retrieved 6-7-2012 from http://www.cbd.int/abs/text/

Cooper GM (2000). Chapter 14: The Eukaryotic Cell Cycle. The cell: a molecular approach (2 ${ }^{\text {nd }}$ ed.). ASM Press. Washington, D.C. ISBN 0-87893-106-6.

Costanzo M, Nishikawa JL, Tang XL, et al. (2004). CDK activity antagonizes Whi5, an inhibitor of G1/S transcription in yeast. Cell 117(7): Jun. 2004. pp. 899-913. DOI: 10.1016/j.cell.2004.05.024

Crick F (1970). Central dogma of molecular biology. Nature 227(5258): pp. 561-563. DOI:10.1038/227561a0.

Cui ZF, Hirata D, Tsuchiya E, et al. (1996). The multidrug resistance-associated protein (MRP) subfamily (Yrs1/Yor1) of Saccharomyces cerevisiae is important for the tolerance to a broad range of organic anions. J. Biol. Chem. 271(25): Jun. 1996. pp. 14712-14716. PMID: 8663018

Curran BPG \& Bugeja V (2005). Basic invesitigations in Saccharomyces cerevisiae. In Yeast Protocols $2^{\text {nd }}$ ed: Methods in molecular biology 313. Xiao W (ed). pp. 1-14. Humana Press. Clifton, NJ. ISBN 1592599583

Davenport J, Manjarrez JR, Peterson L, et al. (2011). Gambogic acid, a natural product inhibitor of Hsp90. Journal of Natural Products 74(5): May 2011. pp. 1085-92. DOI: $10.1021 / \mathrm{np} 200029 \mathrm{q}$

David D, Sundarababu S, Gerst JE, et al. (1998). Involvement of long chain fatty acid elongation in the trafficking of secretory vesicles in yeast. Journal of Cell Biology 143(5): Nov. 1998. pp. 1167-1182. DOI: 10.1083/jcb.143.5.1167

Davidse LC (1986). Benzimidazole fungicides - mechanism of action and biological impact. Annual Review of Phytopathology 24: pp. 43-65. DOI: 10.1146/annurev.phyto.24.1.43

Davidson E \& Levin M (2005). Gene regulatory networks. Proc. Natl. Acad. Sci. USA 102(14): Apr. 2005. pp. 4935-4937. DOI: 10.1073/pnas.0502024102.

Davierwala AP, Haynes J, Li Z, et al. (2005). The synthetic genetic interaction spectrum of essential genes. Nature Genetics 37(10): Oct. 2005. pp. 1147-1152. DOI: 10.1038/ng1640

Davies J \& Jimenez A (1980). A new selective agent for eukaryotic cloning vectors. American Journal of Tropical Medicine and Hygiene 29(5): pp. 1089-1092. PMID: 7001938

De Vries DJ \& Beart PM (1995). Fishing for drugs from the sea: status and strategies. Trends in Pharmacological Sciences 16(8): Aug 1995. pp. 275-279. DOI: 10.1016/S01656147(00)89045-8 
Delahodde A, Delaveau T \& Jacq C (1995). Positive autoregulation of the yeast transcription factor PDR3p, which is involved in control of drug-resistance. Molecular and Cellular Biology 15(8): Aug. 1995. pp. 4043-4051. PMID: 7623800

Di Santo R (2008). Recent patents in antifungal agent discovery. Expert Opinion on Therapeutic Patents 18(3): Mar. 2008. pp. 275-292. DOI: 10.1517/13543776.18.3.275

Diggle CP, Pitt E, Roberts P, et al. (2000). N-3 and n-6 polyunsaturated fatty acids induce cytostasis in human urothelial cells independent of p53 gene function. Journal of Lipid Research 41(9): Sep. 2000. pp. 1509-1515. PMID: 10974058

DiPaola RS (2002). To arrest or not to G(2)-M cell-cycle arrest. Clinical Cancer Research 8(11): Nov. 2002. pp. 3311-3314. PMID: 12429616

Dolinski K, Muir S, Cardenas M, et al. (1997). All cyclophilins and FK506 binding proteins are, individually and collectively, dispensable for viability in Saccharomyces cerevisiae. Proc. Natl. Acad. Sci. USA 94(24): Nov. 1997. pp. 13093-13098. DOI: 10.1073/pnas.94.24.13093

Dolle RE \& Nelson Jr. KH (1999). Comprehensive Survey of Combinatorial Library Synthesis: 1998. Journal of Combinatorial Chemistry 1(4): Jul. 1999. pp. 235-282. DOI: 10.1021/cc9900192

Douglas CM, Dippolito JA, Shei GJ, et al. (1997). Identification of the FKS1 gene of Candida albicans as the essential target of 1,3-beta-D-glucan synthase inhibitors. Antimicrobial Agents and Chemotherapy 41(11): Nov. 1997. pp. 2471-2479.

Douglas CM, Foor F, Marrinan JA, et al. (1994). The saccharomyces-cerevisiae FKS1 (ETG1) gene encodes an integral membrane-protein which is a subunit of 1,3-beta-d-glucan synthase. Proc. Natl. Acad. Sci. USA 91(26): Dec. 1994. pp. 12907-12911. DOI: 10.1073/pnas.91.26.12907

Drews J (2000). Drug discovery: A historical perspective. Science 287(5460): Mar. 2000. pp. 19601964. DOI: $10.1126 /$ science.287.5460.1960

Drickamer K \& Taylor ME (2006). Introduction to Glycobiology (2nd ed.). Oxford University Press. New York, NY, USA. ISBN 978-0-19-928278-4.

Dror V \& Winston F (2004). The Swi/Snf chromatin remodeling complex is required for ribosomal DNA and telomeric silencing in Saccharomyces cerevisiae. Molecular and Cellular Biology 24(18): Sep. 2004. pp. 8227-8235. DOI: 10.1128/MCB.24.18.8227-8235.2004

Drusano G (2001). Pharmacodynamic and pharmacokinetic considerations in antimicrobial selection: focus on telithromycin. Clinical Microbiology and Infection 7(3): pp. 24-29. DOI: 10.1046/j.1469-0691.2001.0070s3024.x

Dudley AM, Janse DM, Tanay A, et al. (2005). A global view of pleiotropy and phenotypically derived gene function in yeast. Molecular Systems Biology 1(1): 2005.0001. DOI: $10.1038 / \mathrm{msb} 4100004$

Edwards MP \& Price DA (2010). Role of physicochemical properties and ligand lipophilicity efficiency in addressing drug safety risks. Annual Reports in Medicinal Chemistry 45: 381391 DOI: 10.1016/S0065-7743(10)45023-X 
Epstein CB \& Cross FR (1992). CLB5 - a novel B-cyclin from budding yeast with a role in S-phase. Genes \& Development 6(9): Sep. 1992. pp. 1695-1706. DOI: 10.1101/gad.6.9.1695

Escalera J, von Hehn CA, Bessac BF, et al. (2008). TRPA1 mediates the noxious effects of natural sesquiterpene deterrents. J. Biol. Chem. 283(35): Aug. 29. pp. 24136-24144. DOI: 10.1074/jbc.M710280200

Evans DA, Welch DS, Speed ATG, et al. (2009). An aldol-based synthesis of (+)-peloruside A, a potent microtubule stabilizing agent. Journal of the American Chemical Society 131 (11): Mar 2009. pp. 3840-3841. DOI: 10.1021/ja900020a

Fabricant DS \& Farnsworth NR (2001). The value of plants used in traditional medicine for drug discovery. Environ Health Perspect 109(1): pp. 69-75. PMCID: PMC1240543

Fang H, Mullins C \& Green N (1997). In addition to SEC11, a newly identified gene, SPC3, is essential for signal peptidase activity in the yeast endoplasmic reticulum. J. Biol. Chem. 272(20): May 1997. pp. 13152-13158. DOI: 10.1074/jbc.272.20.13152

Faulkner JD, Newman DJ \& Cragg GM (2004). Investigations of the marine flora and fauna of the Islands of Palau. Nat Prod Rep 21 (1): Feb. 2004. pp. 50-76. DOI: 10.1039/b300664f.

Feher M \& Schmidt JM (2003). Property distributions: differences between drugs, natural products, and molecules from combinatorial chemistry. Journal of Chemical Information and Computer Sciences 43(1): Jan. 2003. pp. 218-227. DOI: 10.1021/ci0200467

Fields S \& Johnston M (2005). Cell biology: Whither model organism research? Science 307 (5717): Mar. 2005. pp. 1885-6. DOI:10.1126/science.1108872.

Forsburg SL (2001). The art and design of genetic screens: yeast. Nature Reviews Genetics 2(9): Sep. 2001. pp. 659-668. DOI: 10.1038/35088500

Foury F (1997). Human genetic diseases: a cross-talk between man and yeast. Gene 195(1): Aug. 1997. pp. 1-10. DOI: 10.1016/S0378-1119(97)00140-6.

Fred Hutchinson Cancer Research Center (2012). Cytotoxic agents. Retrieved 29-8-2-12 from http://extranet.fhcrc.org/EN/sections/ehs/hamm/chap3/section12/12.12 cytotoxic.html

Fried HM \& Warner JR (1981). Cloning of yeast gene for trichodermin resistance and ribosomal protein-L3. Proc. Natl. Acad. Sci. USA -Biological Sciences 78(1): Jan. 1981. pp. 238-242. DOI: $10.1073 /$ pnas.78.1.238

Fried HM \& Warner JR (1982). Molecular-cloning and analysis of yeast gene for cycloheximide resistance and ribosomal-protein L29. Nucleic Acids Research 10(10): Jan. 1982. pp. 31333148. DOI: $10.1093 /$ nar/10.10.3133

Fuller RS, Brake A \& Thorner J (1989). Yeast prohormone processing enzyme (KEX2 gene-product) is a Ca-2+-dependent serine protease. Proc. Natl. Acad. Sci. USA 86(5): Mar.1989. pp. 1434-1438. DOI: 10.1073/pnas.86.5.1434

Fullwood MJ, Wei CL, Liu ET, et al. (2009). Next-generation DNA sequencing of paired-end tags (PET) for transcriptome and genome analyses. Genome Research 19(4): Apr. 2009. pp. 521-532. DOI: 10.1101/gr.074906.107 
Funakoshi Y \& Suzuki T (2009). Glycobiology in the cytosol: The bitter side of a sweet world. Biochim. Biophys. Acta 1790 (2): Jan. 2009. pp. 81-94. DOI: 10.1016/j.bbagen.2008.09.009. PMID 18952151

Gassner NC, Tamble CM, Bock JE, et al. (2007). Accelerating the discovery of biologically active small molecules using a high-throughput yeast halo assay. Journal of Natural Products 70(3): Mar. 2007. pp. 383-390. DOI: 10.1021/np060555t.

Gershenzon J \& Dudareva N (2007). The function of terpene natural products in the natural world. Nature Chemical Biology 3(7): Jul. 2007. 408-414. DOI: 10.1038/nchembio.2007.5

Ghose AK, Viswanadhan VN \& Wendoloski JJ (1999). A knowledge-based approach in designing combinatorial or medicinal chemistry libraries for drug discovery. Journal of Combinatorial Chemistry 1(1): Jan. 1999. pp. 55-68. DOI:10.1021/cc9800071.

Giaever G, Chu AM, Ni L, et al. (2002). Functional profiling of the Saccharomyces cerevisiae genome. Nature 418(6896): Jul. 2002. pp. 387-391. DOI: 10.1038/nature00935

Giaever G, Flaherty P \& Arkin AP (2003). Chemical genomics in yeast via Haploinsufficiency Profiling (HIP). Yeast 20(1): pp. S18-S18. DOI: 10.1073/pnas.0307490100

Gibbs JB (2000). Mechanism-based target identification and drug discovery in cancer research. Science 287(5460) Mar. 2000. pp. 1969-1973. DOI: 10.1126/science.287.5460.1969

Gietz RD \& Schiestl RH (2008). High-efficiency yeast transformation using LiAc/SS carrier DNA/PEG method. Nature Protocols 2(1): Nov. 2008. pp. 31-34. DOI: 10.1038/nprot.2007.13

Goffeau A, Barrell BG, Bussey H, et al. ( 1996). Life with 6000 genes. Science 274 (5287): Oct. 1996. pp. 546, 563-567. DOI: 10.1126/science.274.5287.546

Goldstein AL \& McCusker JH (1999). Three new dominant drug resistance cassettes for gene disruption in Saccharomyces cerevisiae. Yeast 15(14): Oct. 1999. pp. 1541-1553. DOI: 10.1002/(SICI)1097-0061(199910)15:14<1541::AID-YEA476>3.0.CO;2-K

Gordeeva E, Lushniknov DE \& Zefirov NF (1992). COMPASS Program - an original semi-emperical approach to computer-assisted synthesis. Tetrahedron 48(18): pp. 3789-3804. DOI: 10.1016/S0040-4020(01)92270-7.

Gray JW, Dolbeare F, Pallavicini MG, et al. (1986). Cell-cycle analysis using flow-cytometry. International Journal of Radiation Biology 49(2): Feb. 1986. pp. 237-255. DOI: 10.1080/09553008514552531

Groen AK (2001). The pros and cons of gene expression analysis by microarrays. Journal of Hepatology 35(2): Aug. 2001. pp. 295-296. DOI: 10.1016/S0168-8278(01)00156-8

Gruber JD, Vogel K, Kalay G, et al. (2012). Contrasting properties of gene-specific regulatory, coding, and copy number mutations in Saccharomyces cerevisiae: Frequency, effects, and dominance. PLoS Genetics 8(2): Feb. 2012. Art. e1002497. DOI: 10.1371/journal.pgen.1002497 
Gullo VP, McAlpine J, Lam KS, et al. (2006). Drug discovery from natural products. Journal of Industrial Microbiology \& Biotechnology 33(7): Jul. 2006. pp. 523-531. DOI: 10.1007/s10295-006-0107-2

Haase SB \& Reed SI (2002). Improved flow cytometric analysis of the budding yeast cell cycle. Cell Cycle 1(2): Mar-Apr 2002. pp. 132-136. PMID: 12429922

Hagiwara H \& Sunada Y (2004). Mechanism of taxane neurotoxicity. Breast cancer 11(1): pp. 8285. DOI: $10.1007 / \mathrm{BF} 02968008$

Hall JL (2002). Cellular mechanisms for heavy metal detoxification and tolerance. Journal of Experimental Botany 53(366): Jan. 2002. pp. 1-11. DOI: 10.1093/jexbot/53.366.1

Hanrahan J \& Snyder M (2003). Cytoskeletal activation of a checkpoint kinase. Molecular Cell 12(3): Sep. 2003. pp. 663-673. DOI: 10.1016/j.molcel.2003.08.006

Hartman JL, Garvik B, Hartwell L. (2001). Principles for the buffering of genetic variation. Science 291(5506): Feb. 2001. pp. 1001-1004. DOI: 10.1126/science.291.5506.1001

Hartwell L, Culotti J \& Reid B (1970). Genetic control of the cell division cycle in yeast. Part I: Detection of mutants. Proc. Natl. Acad. Sci. USA 66(2): pp. 352-359. DOI: 10.1073/pnas.66.2.352

Hartwell LH (2002) Yeast and cancer: A Nobel lecture. Bioscience Reports 22(3-4): Jun. - Aug. 2002. pp. 373-394. DOI: 10.1023/A:1020918107706

Heidler S \& Radding J (1995). The AUR1 gene in Saccharomyces cerevisiae encodes dominant resistance to the antifungal agent aureobasidin A (LY295337). Antimicrobial Agents and Chemotherapy 39(12): Dec. 1995. pp. 2765-2769. DOI: 10.1128/AAC.39.12.2765.

Heitman J, Movva NR \& Hall MN (1991). Targets for cell-cycle arrest by the immunosuppressant rapamycin in yeast. Science 253(5022): Aug. 1991. pp. 905-909. DOI: 10.1126/science.1715094

Herskowitz I (1988). Life-cycle of the budding yeast Saccharomyces cerevisiae. Microbiological Reviews 52(4): Dec. 1988. pp. 536-553. PMID: 3070323

Hieter P, Mann C, Snyder M \& Davis RW (1985). Mitotic stability of yeast chromosomes: a colony color assay that measures nondisjunction and chromosome loss. Cell 40(2): pp. 381-382. DOI: 10.1016/0092-8674(85)90152-7

Hillenmeyer ME, Fung E, Wildenhain J, et al. (2008). The chemical genomic portrait of yeast: Uncovering a phenotype for all genes. Science 320(5874): Apr. 2008. pp. 362-365. DOI: 10.1126/science. 1150021

Ho CH, Magtanong L, Barjer SL, et al. (2009). A molecular barcoded yeast ORF library enables mode-of-action analysis of bioactive compounds. Nature Biotechnology 27(4): Apr. 2009. pp. 369 - 377. DOI:10.1038/nbt.1534

Ho CH, Piotrowski J, Dixon S, et al. (2011). Combining functional genomics and chemical biology to identify targets of bioactive compounds. Current Opinion in Chemical Biology 15(1): Feb. 2011. pp. 66-78. DOI: 10.1016/j.cbpa.2010.10.023 
Hood KA, West LM, Rouwe B, et al. (2002). Peloruside A, a novel antimitotic agent with paclitaxel-like microtubule-stabilizing activity. Cancer Research 62(12): Jun. 2002. pp. 3356-3360.

Hood-DeGrenier JK (2008). A western blot-based investigation of the yeast secretory pathway designed for an intermediate-level undergraduate cell biology laboratory. CBE-Life Sciences Education 7(1): pp. 107-117. DOI: 10.1187/cbe.07-07-0047

Hoon S, Smith AM, Wallace IM, et al. (2008). An integrated platform of genomic assays reveals small-molecule bioactivities. Nature Chemical Biology 4(8): Aug. 2008. pp. 498-506. DOI: 10.1038/nchembio.100

Huang PH \& McBride WG (1997). Interaction of [glutarimide-2-14C]-thalidomide with rat embryonic DNA in vivo. Teratogenesis, Carcinogenesis, and Mutagenesis 17(1): pp. 1-5. DOI:10.1002/(SICI)1520-6866.17:1<1::AID-TCM2>3.0.CO;2-L.

Hughes JD, Blagg J, Price DA, et al. (2008). Physiochemical drug properties associated with in vivo toxicological outcomes. Bioorganic \& Medicinal Chemistry Letters 18(17): Sep. 2008. pp. 4872-4875. DOI: 10.1016/j.bmcl.2008.07.071

Huh WK, Falvo JV, Gerke LC, et al. (2003). Global analysis of protein localization in budding yeast. Nature 425(6959): Oct. 2003. pp. 686-691. DOI: 10.1038/nature02026

Iken K, Avila C, Ciavatta ML, et al. (1998). Hodgsonal, a new drimane sesquiterpene from the mantle of the Antarctic nudibranch Bathydoris hodgsoni. Tetrahedron Letters 39(31): Jul. 1998. pp. 5635-5638. DOI: 10.1016/S0040-4039(98)01095-8

International Council for Science / Conseil International pour la Science (2002). "Science and Traditional Knowledge: Report from the ICSU Study Group on Science and Traditional Knowledge". p. 3. Retrieved 24-5-2012 from http://www.icsu.org/publications/reportsand-reviews/science-traditional-knowledge/Science-traditional-knowledge.pdf

Jackson JR, Gilmartin A, Imburgia C, et al. (2000). An indolocarbazole inhibitor of human checkpoint kinase (Chk1) abrogates cell cycle arrest caused by DNA damage. Cancer Research 60(3): Feb. 2000. pp. 566-572. PMID: 10676638

Jayarajan R \& Vasuki G (2012). Building libraries of skeletally diverse scaffolds from novel heterocyclic active methylene compound through multi-component reactions. Tetrahedron Letters 53 (24): Jun. 2012. pp. 3044-3048. DOI: 10.1016/j.tetlet.2012.04.013.

Jefford CW \& Rinehart KL (1998). Pharmaceuticals and the Sea. Shield LS, (ed). Technomic Publishing Co. Lancaster, PA. ISBN: 0-87762-581-6

Jelinsky SA \& Samson LD (1999). Global response of Saccharomyces cerevisiae to an alkylating agent. Proc. Natl. Acad. Sci. USA 96(4): Feb. 1999. pp. 1486-1491. DOI: 10.1073/pnas.96.4.1486

Jin R, Dobry CJ, McCown PJ, et al. (2008). Large-scale analysis of yeast filamentous growth by systematic gene disruption and overexpression. Molecular Biology of the Cell 19(1): Jan. 2008. pp. 284-296. DOI: 10.1091/mbc.E07-05-0519

Jochum A, Jackson D, Schwarz $\mathrm{H}$, et al. (2002). Yeast $Y s / 2 p$, homologous to Sec7 domain guanine nucleotide exchange factors, functions in endocytosis and maintenance of vacuole 
integrity and interacts with the Arf-like small GTPase Arl1p. Molecular and Cellular Biology 22(13): Jul. 2002. pp. 4914-4928. DOI: 10.1128/MCB.22.13.4914-4928.2002

Jones DGL, Reusser U \& Braus GH (1991). Molecular-cloning, characterization and analysis of the regulation of the ARO2 gene, encoding chorismate synthase, of Saccharomycescerevisiae. Molecular Microbiology 5(9): Sep. 1991. pp. 2143-2152. DOI: 10.1111/j.13652958.1991.tb02144.x

Jones EW \& Fink GR (1982). Regulation of amino acid and nucleotide biosynthesis in yeast. In The Molecular Biology of the Yeast Saccharomyces: Metabolism and Gene Expression. Strathern JN, Jones EW \& Broach JR eds. pp.181-299. Cold Spring Harbor Laboratory Press. Cold Spring Harbor, NY. ISBN-10: 0879691492

Jonikas MC, Collins SR, Denic V, et al. (2009). Comprehensive characterization of genes required for protein folding in the endoplasmic reticulum. Science 323(1693): Mar. 2009. pp. 16931697. DOI: 10.1126/science.1167983

Jordan MA \& Wilson L (2004). Microtubules as a target for anticancer drugs. Nature Reviews Cancer 4(4): Apr. 2004. pp. 253-265. DOI: 10.1038/nr1317

Jorgensen P, Nelson B, Robinson MD, et al. (2002). High-resolution genetic mapping with ordered arrays of Saccharomyces cerevisiae deletion mutants. Genetics 162(3): Nov. 2002. pp. 1091-1099.

Justice MC, Hsu MJ, Tse B, et al. (1998). Elongation factor 2 as a novel target for selective inhibition of fungal protein synthesis. Journal of Biological Chemistry 273(6): Feb. 1998. pp. 3148-3151. DOI: 10.1074/jbc.273.6

Kahan FM, Kahan JS, Cassidy PJ, et al. (1974). Mechanism of action of fosfomycin (phosphonomycin). Annals of the New York Academy of Sciences 235: May 1974. pp. 364386. DOI: 10.1111/j.1749-6632.1974.tb43277.x

Kapitzky L, Beltrao P, Berens TJ, et al. (2010). Cross-species chemogenomic profiling reveals evolutionarily conserved drug mode of action. Molecular Systems Biology 6: Dec.2010. Art. 451. DOI: 10.1038/msb.2010.107

Karp G (2005). Cytoplasmic membrane systems: structure, function, and membrane trafficking. In Cellular and Molecular Biology: Concepts and Experiments. pp. 279-333. John Wiley \& Sons. Hoboken, NJ. ISBN-10: 1118206738

Katzmann DJ, Hallstrom TC, Voet M, et al. (1995). Expression of an ATP-binding cassette transporter-encoding gene (YOR1) is required for oligomycin resistance in Saccharomyces cerevisiae. Molecular and Cellular Biology 15(12): Dec. 1995. pp. 6875-6883. PMID: 8524254

Kaufman RJ (2002). Orchestrating the unfolded protein response in health and disease. Journal of Clinical Investigation 110(10): Nov. 2002. pp. 1389-1398. DOI: 10.1172/JC1200216886

Kile BT \& Hilton DJ. (2005) The art and design of genetic screens: mouse. Nature Reviews Genetics 6(7): Jul. 2005. pp. 557-567. DOI: 10.1038/nrg1636

Kishi T, Watanabe T \& Folkers K (1977). Bioenergetics in clinical medicine XV. Inhibition of coenzyme Q10-enzymes by clinically used adrenergic blockers of beta-receptors. Research 
communications in chemical pathology and pharmacology 17(1): pp. 157-164. PMID 17892.

Knight J (2001). When the chips are down. Nature 410(6831): Apr. 2001. pp. 860-861. DOI: $10.1038 / 35073680$

Koch HP \& Czejka MJ (1986). Evidence for the intercalation of thalidomide into DNA: clue to the molecular mechanism of thalidomide teratogenicity?. Z. Naturforsch. C, J. Biosci. 41(1112): Nov. 1986. pp. 1057-61. PMID 2953123.

Koehn FE \& Carter GT (2005). The evolving role of natural products in drug discovery. Nature Reviews Drug Discovery 4(3): Mar. 2005. pp. 206-220. DOI: 10.1038/nrd1657

Kohanski MA, Dwyer DJ, Hayete B, et al. (2007). A common mechanism of cellular death induced by bactericidal antibiotics. Cell 130(5): Sep. 2007. pp. 797-810. DOI: 10.1016/j.cell.2007.06.049

Lawley PD (1980). DNA as a target of alkylating carcinogens. British Medical Bulletin 36(1): pp. 19-24. PMID: 7020825

Lawrence $\mathrm{CL}$, Botting $\mathrm{CH}$, Antrobus R, et al. (2004). Evidence of a new role for the high-osmolarity glycerol mitogen-activated protein kinase pathway in yeast: Regulating adaptation to citric acid stress. Molecular and Cellular Biology 24(8): Apr. 2004. pp. 3307-3323. DOI: 10.1128/MCB.24.8.3307.3323.2004

Lee MLT, Kuo FC, Whitmore GA, et al. (2000). Importance of replication in microarray gene expression studies: Statistical methods and evidence from repetitive CDNA hybridizations. Proc. Natl. Acad. Sci. USA 97(18): Aug. 200. pp. 9834-9839. DOI: 10.1073/pnas.97.18.9834

Leeson PD \& Springthorpe B (2007). The influence of drug-like concepts on decision-making in medicinal chemistry. Nature Reviews Drug Discovery 6(11): Nov 2007. pp. 881-890. DOI: $10.1038 / \mathrm{nrd} 2445$

Leppert G, Mcdevitt R, Falco SC, et al. (1990). Cloning by gene amplification of 2 loci conferring multiple-drug resistance in Saccharomyces. Genetics 125(1): May 1990. pp. 13-20. PMID: 2160400

Levy SF \& Siegal ML (2008). Network hubs buffer environmental variation in Saccharomyces cerevisiae. PLoS Biol 6(11): Nov. 2008. pp. 2588-2604. doi:10.1371/journal.pbio.0060264

Lewington S, Whitlock G, Clarke R, et al. (2007). Blood cholesterol and vascular mortality by age, sex, and blood pressure: a meta-analysis of individual data from 61 prospective studies with 55,000 vascular deaths. Lancet 370(9602): Dec. 2007. pp. 1829-39. DOI: 10.1016/S0140-6736(07)61778-4

Li JWH \& Vederas JC (2009). Drug discovery and natural products: End of an era or an endless frontier? Science 325(5937): Jul. 2009. pp. 161-165. DOI: 10.1126/science.1168243

Li K, Ouyang $\mathrm{H}$, Lue $\mathrm{Y}$, et al. (2011). Repression of N-glycosylation triggers the unfolded protein response (UPR) and overexpression of cell wall protein and chitin in Aspergillus fumigates. Microbiology-SGM 157: Jul. 2011. pp. 1968-1979. DOI: 10.1099/mic.0.047712-0 
Lindsay MA (2003). Innovation - Target discovery. Nature Reviews Drug Discovery 2(10): Oct. 2003. pp. 831-838. DOI: $10.1038 / \mathrm{nrd} 1202$

Lipinski CA, Lombardo F, Dominy BW \& Feeney PJ (2001). Experimental and computational approaches to estimate solubility and permeability in drug discovery and development settings. Advanced Drug Delivery Reviews 46(1-3): Mar. 2001. pp. 3-26. DOI: 10.1016/S0169-409X(00)00129-0

Little MA \& Jones NS (2011). Generalized methods and solvers for noise removal from piecewise constant signals: I. Background theory. Proceedings of the Royal Society A-Mathematical Physical and Engineering Sciences 467(2135): Nov. 2011. 3088-3114. DOI: 10.1098/rspa.2010.0671

Liu Y, Vidanes G, Lin YC, et al. (2000). Characterization of a Saccharomyces cerevisiae homologue of Schizosaccharomyces pombe Chk1 involved in DNA-damage-induced M-phase arrest. Molecular and General Genetics 262(6): Jan. 2000. pp. 1132-1146. DOI: 10.1007/PL00008656

Liu YX, Hsiung YC, Jannatipour M, et al. (1994). Yeast topoisomerase-ii mutants resistant to antitopoisomerase agents - identification and characterization of new yeast topoisomerase-II mutants selected for resistance to etoposide. Cancer Research 54(11): Jun. 1994. pp. 2943-2951.

Lodish H \& Berk A (2003) The secretory pathway. In Molecular Cell Biology, $5^{\text {th }}$ ed. Scott MP (ed). W. H. Freeman. London, UK. ISBN: 0716743663

Lodish H, Berk A, Zipursky SL, et al. (2000). Mutations: Types and Causes. In Molecular Cell Biology $4^{\text {th }}$ ed. pp. 198-204. W. H. Freeman. New York, NY. ISBN-10: 0716731363

Loken MR (1990). Immunofluorescence techniques in flow cytometry and sorting (2nd ed.). pp. 341-53. John Wiley \& Sons. New York, NY.

Luesch $\mathrm{H}, \mathrm{Wu}$ TYH, Ren PD, et al. (2005). A genome-wide overexpression screen in yeast for small-molecule target identification. Chemistry \& Biology 12(1): Jan. 2005. pp. 55-63. DOI: 10.1016/j.chembiol.2004.10.015

Luttik MAH, Kotter P, Salomons FA, et al. (2000). The Saccharomyces cerevisiae ICL2 gene encodes a mitochondrial 2-methylisocitrate lyase involved in propionyl-coenzyme $A$ metabolism. Journal of Bacteriology 182(24): Dec. 2000. 7007-7013. DOI: 10.1128/JB.182.24.7007-7013.2000

Macklis JD \& Madison RD (1990). Progressive incorporation of propidium iodide in cultured mouse neurons correlates with declining electrophysiological status - a fluorescence scale of membrane integrity. Journal of Neuroscience Methods 31(1): Jan. 1990. pp. 43-46. DOI: 10.1016/0165-0270(90)90007-3

Maere S, Heymans K \& Kuiper M (2005). BiNGO: a Cytoscape plugin to assess overrepresentation of Gene Ontology categories in Biological Networks. Bioinformatics 21(16): Aug. 2005. pp. 3448-3449. DOI: 10.1093/bioinformatics/bti551

Mager WH \& Winderickx J (2005). Yeast as a model for medical and medicinal research. Trends in Pharmacological Sciences 26(5): May 2005. pp. 265-273. DOI: 10.1016/j.tips.2005.03.004 
March J \& Smith D (2001). Advanced organic chemistry, 5th ed. John Wiley \& Sons. New York, NY. ISBN-10: 0471720917

Marcoff L \& Thompson PD (2007). The role of coenzyme Q10 in statin-associated myopathy: a systematic review. Journal of the American College of Cardiology. 49(23): Jun. 2007. pp. 2231-2237. DOI: 10.1016/j.jacc.2007.02.049.

Markovich S, Yekutiel A, Shalit I, et al. (2004). Genomic approach to identification of mutations affecting caspofungin susceptibility in Saccharomyces cerevisiae. Antimicrobial Agents and Chemotherapy. 48(10): Oct 2004. pp. 3871-3876. DOI: 10.1128/AAC.48.10.38713876.2004

Maser P, Sutterlin C, Kralli A, et al. (1999). A nucleoside transporter from Trypanosoma brucei involved in drug resistance. Science 285(5425). Jul. 1999. pp. 242-244. DOI: $10.1126 /$ science. 285.5425 .242

McInnes C \& Sykes BD (1997). Growth factor receptors: Structure, mechanism, and drug discovery. Biopolymers 43(5): pp. 339-366. DOI: 10.1002/(SICI)10970282(1997)43:5<339::AID-BIP2>3.0.CO;2-W

McLellan JL, O'Neil NJ, Barrett I, et al. (2012). Synthetic lethality of cohesins with PARPs and replication fork mediators. PLOS Genetics 8(3): Mar 2012. Art. e1002574. DOI: 10.1371/journal.pgen.1002574

Mendenhall MD \& Hodge AE (1998). Regulation of cdc28 cyclin-dependent protein kinase activity during the cell cycle of the yeast Saccharomyces cerevisiae. Microbiology and Molecular Biology Reviews 62(4): Dec. 1998. pp. 1191-\&. PMID: 9841670

Mertins SD, Myers TG, Holbeck SL, et al. (2004). In vitro evaluation of dimethane sulfonate analogues with potential alkylating activity and selective renal cell carcinoma cytotoxicity. Molecular Cancer Therapeutics 3(7): Jul. 2004. pp. 849-860. PMID: 15252146

Meusser B, Hirsch C, Jarosch E, et al. (2005). ERAD: the long road to destruction. Nature Cell Biology 7(8): Aug. 2005. pp. 766-772. DOI: 10.1038/ncb0805-766

Mewes HW, Frishman D, Guldener U, et al. (2002). MIPS: a database for genomes and protein sequences. Nucleic Acids Research 50(1): Jan. 2002. pp. 31-34. DOI: 10.1093/nar/30.1.31

Meyers S, Schauer W, Balzi E, et al. (1992). Interaction of the yeast pleiotropic drug-resistance gene-PDR1 and gene-PDR5. Current Genetics 21(6). May 1992. pp. 431-436. DOI: 10.1007/BF00351651

Minear S, O'Donnell AF, Ballew A, et al. (2011). Curcumin inhibits growth of Saccharomyces cerevisiae through iron chelation. Eukaryotic Cell 10(11): Nov. 2011. pp. 1574-1581. DOI: 10.1128/EC.05163-11

Misu K, Fujimura-Kamada K, Ueda T, et al. (2003). Cdc50p, a conserved endosomal membrane protein, controls polarized growth in Saccharomyces cerevisiae. Molecular Biology of the Cell 14(2): Feb. 2003. pp. 730-747. DOI: 10.1091/mbc.E02-06-0314

Miyamoto Y, Machida K, Mizunuma M, et al. (2002). Identification of Saccharomyces cerevisiae isoleucyl-tRNA synthetase as a target of the $G(1)$-specific inhibitor reveromycin $A$. Journal 
of Biological Chemistry 277(32): Aug. 2002. pp. 28810-28814. DOI: 10.1074/jbc.M203827200

Mooberry SL, Tien G, Hernandez AH, et al. (1999). Laulimalide and isolaulimalide, new paclitaxellike microtubule stabilizing agents. Cancer Research 59(3): Feb. 1999. pp. 653-660.

Morrissey JP \& Osbourn AE (1999). Fungal resistance to plant antibiotics as a mechanism of pathogenesis. Microbiology and Molecular Biology Reviews 63(3): Sep. 1999. pp. 708-\&. PMID: 10477313

Munkacsi $A B$, Chen FW, Brinkman MA, et al. (2011). An "exacerbate-reverse" strategy in yeast identifies histone deacetylase inhibition as a correction for cholesterol and sphingolipid transport defects in human Niemann-Pick type C disease. Journal of Biological Chemistry 286(27): Jul. 2011. pp. 23842-23851. DOI: 10.1074/jbc.M111.227645

Murray RK, Granner DK \& Rodwell RW (2006). Harper's Illustrated Biochemistry 27th Ed., McGraw-Hill. New York, NY. ISBN-10: 007176576X

New Zealand Institute of Chemistry (2012). The design and development of anticancer drugs. Retrieved 13-9-2012 from http://nzic.org.nz/ChemProcesses/biotech/12J.pdf

Newman D \& Cragg G (2007). Natural products as sources of new drugs over the last 25 years. Journal of Natural Products 70(3): Mar. 2007. pp. 461-477. DOI: 10.1021/np068054v

Newton MA, Kendziorski CM, Richmond CS, et al. (2001). On differential variability of expression ratios: Improving statistical inference about gene expression changes from microarray data. Journal of Computational Biology 8(1): pp. 37-52. DOI: $10.1089 / 106652701300099074$

Nislow C \& Giaever G (2007). Chemical genomic tools for understanding gene function and drug action. Methods in Microbiology 36. Stansfield I \& Stark MJ (eds.) pp. 387-401. DOI: 10.1016/S0580-9517(06)36017-5

Nurse P, Thuriaux P, Nasmyth K, (1976). Genetic-control of cell-division cycle in fission yeast Schizosaccharomyces-pombe. Molecular \& General Genetics 146(2): pp. 167-178. DOI: 10.1007/BF00268085

Okamuraa K, Kimataa Y, Higashioa $\mathrm{H}$, et al. (2000). Dissociation of Kar2p/BiP from an ER sensory molecule, Ire1p, triggers the unfolded protein response in yeast. Biochemical and Biophysical Research Communications 279(2): Dec. 2000. pp. 445-450. DOI: 10.1006/bbrc.2000.3987

O'Neill AJ, Miller K, Oliva B, et al. (2004). Comparison of assays for detection of agents causing membrane damage in Staphylococcus aureus. Journal of Antimicrobial Chemotherapy 54(6): Dec. 2004. pp. 1127-1129. DOI: 10.1093/jac/dkh476

Parodi AJ (2000). Role of $\mathrm{N}$-oligosaccharide endoplasmic reticulum processing reactions in glycoprotein folding and degradation. Biochemical Journal 348(1): May 2000. pp. 1-13. DOI: 10.1042/0264-6021:3480001

Parsons $A B$, Brost RL, Ding HM, et al. (2004). Integration of chemical-genetic and genetic interaction data links bioactive compounds to cellular target pathways. Nature Biotechnology 22(1): Jan. 2004. pp. 62-69. DOI: 10.1038/nbt919 
Parsons AB, Lopez A, Givoni IE, et al. (2006). Exploring the mode-of-action of bioactive compounds by chemical-genetic profiling in yeast. Cell 126(3): Aug. 2006. pp. 611-625. DOI: 10.1016/j.cell.2006.06.040

Patterson AV, Ferry DM, Edmunds SJ, et al. (2007). Mechanism of action and preclinical antitumor activity of the novel hypoxia-activated DNA cross-linking agent PR-104. Clinical Cancer Research 13(13): Jul. 2007. pp. 3922-3932. DOI: 10.1158/1078-0432.CCR-07-0478

Paul SM, Mytelka DS, Dunwiddie CT, et al. (2010). How to improve R\&D productivity: the pharmaceutical industry's grand challenge. Nature Reviews Drug Discovery 9(3):Mar. 2010. pp. 203-214. DOI: $10.1038 / \mathrm{nrd} 3078$

Pea F, Viale P, Pavan F, et al. (2007). Pharmacokinetic considerations for antimicrobial therapy in patients receiving renal replacement therapy. Clinical Pharmacokinetics 46(12): pp. 997138. PMID: 18027987

Pervin S, Singh R \& Chaudhuri G (2001). Nitric oxide-induced cytostasis and cell cycle arrest of a human breast cancer cell line (MDA-MB-231): Potential role of cyclin D1. Proc. Natl. Acad. Sci. USA 98(6): Mar. 2001. pp. 3583-3588 DOI: 10.1073/pnas.041603998

Peterson CL, Cote J, Quinn J, et al. (1995). The SWI-SNF complex - a chromatin remodeling machine. Journal of Cellular Biochemistry Suppl. 21B: Apr. 1995. pp. 154-154. DOI: 10.1016/S0968-0004(00)88990-2

Pike BL, Yongkiettrakul S, Tsai MD, et al. (2004). Mdt1, a novel Rad53 FHA1 domain-interacting protein, modulates DNA damage tolerance and $G(2) / M$ cell cycle progression in Saccharomyces cerevisiae. Molecular and Cellular Biology 24(7): Apr. 2004. pp. 27792788. DOI: 10.1128/MCB.24.7.2779-2788.2004

Poulin R, Lu L, Ackermann B, et al. (1992). Mechanism of the irreversible inactivation of mouse ornithine decarboxylase by alpha-difluoromethylornithine. Characterization of sequences at the inhibitor and coenzyme binding sites. The Journal of Biological Chemistry 267 (1): pp. 150-158. PMID 1730582

Prakash S, Sung P \& Prakash L (1993). DNA-repair genes and proteins of Saccharomycescerevisiae. Annual Review of Genetics 27: pp. 33-70. DOI: 10.1146/annurev.ge.27.120193.000341

Prinz S, Avila-Campillo I, Aldridge C, et al. (2004). Control of yeast filamentous-form growth by modules in an integrated molecular network. Genome Research 14(3): Mar. 2004. pp. 380-390. DOI: 10.1101/gr.2020604

Pryor DE, O'Brate A, Bilcer G, et al. (2002). The microtubule stabilizing agent laulimalide does not bind in the taxoid site, kills cells resistant to paclitaxel and epothilones, and may not require its epoxide moiety for activity. Biochemistry 41(29): Jun. 2002. pp. pp 9109-9115. DOI: $10.1021 / \mathrm{bi020211b}$

PubChem (2012). BioActivity Analysis: propyl hept-2-ynoate. Retrieved 26-9-2012 from http://pubchem.ncbi.nlm.nih.gov/assay/assay.cgi?q=r\&version=1.1\&reqid=15672396162 $\underline{55425332}$ 
Puig S, Lee J, Lau M, et al. (2002). Biochemical and genetic analyses of yeast and human high affinity copper transporters suggest a conserved mechanism for copper uptake. J. Biol. Chem. 277(29): Jul. 2002. pp. 26021-26030. DOI: 10.1074/jbc.M202547200

Quek NCH (2011). The characterisation of TA-289, a novel antifungal from Fusarium sp. Unpublished thesis for masters degree. Victoria University of Wellington. Wellington, NZ.

Reiss G, Heesen ST, Zimmerman J, et al. (1996). Isolation of the ALG6 locus of Saccharomyces cerevisiae required for glucosylation in the $N$-linked glycosylation pathway. Glycobiology 6(5): Jul. 1996. pp. 493-498. DOI: 10.1093/glycob/6.5.493

Rine J (1991). Gene overexpression in studies of Saccharomyces cerevisiae. Methods Enzymol. 194: pp. 239-251. PMID: 2005790

Roberg KJ, Bickel S, Rowley N, et al. (1997). Control of amino acid permease sorting in the late secretory pathway of Saccharomyces cerevisiae by SEC13, LST4, LST7 and LST8. Genetics 147(4): Dec. 1997. pp. 1569-1584. PMID: 9409822

Rockman HB (2004). Intellectual property law for engineers and scientists. p. 94. IEEE Antennas and Propagation Society, Wiley-IEEE. New York, NY. ISBN: 0471449989.

Roemer T, Davies J, Giaever G \& Nislow C (2012). Bugs, drugs and chemical genomics. Nature Chemical Biology 8. Jan. 2012. pp. 46-56. DOI: 10.1038/nchembio.744

Rosenber B, Vancamp L \& Krigas T (1965). Inhibition of cell division in Escherichia coli by electrolysis products from a platinum electrode. Nature 205(4972): pp. 698-\& DOI: $10.1038 / 205698 \mathrm{a0}$

Rosenthal GA (1986). Biochemical insight into insecticidal properties of L-canavanine, a higherplant protective allelochemical. Journal of Chemical Ecology 12(5): May 1986. pp. 11451156. DOI: 10.1007/BF01639001

Runge KW \& Robbins PW (1986). A new yeast mutation in the glucosylation steps of the asparagine-linked glycosylation pathway - formation of a novel asparagine-linked oligosaccharide containing 2 glucose residues. J. Biol. Chem. 261(33): Nov. 1986. pp. 5582-5590. PMID: 3536907

Sams-Dodd F (2005). Target-based drug discovery: is something wrong?. Drug Discovery Today 10(2): Jan. 2005. pp. 139-147. DOI: 10.1016/S1359-6446(04)03316-1.

Sams-Dodd F (2006). Drug discovery: selecting the optimal approach. Drug Discovery Today 11(910): May 2006. pp. 465-472. DOI: 10.1016/j.drudis.2006.03.015

Schena M, Heller RA, Theriault TP, et al. (1998). Microarrays: biotechnology's discovery platform for functional genomics. Trends in Biotechnology 16(7): Jul. 1998. pp. 301-306. DOI: 10.1016/S0167-7799(98)01219-0

Schlacher K, Pham P, Cox MM, et al. (2006). Roles of DNA polymerase $V$ and RecA protein in SOS damage-induced mutation. Chemical Reviews 106(2): Feb. 2006. pp. 406-419. DOI: $10.1021 /$ cr0404951 
Schnappinger D \& Hillen W (1996). Tetracyclines: Antibiotic action, uptake, and resistance mechanisms. Archives of Microbiology 165(6): Jun. 1996. pp. 359-369. DOI: $10.1007 /$ s002030050339

Schneider-Poetsch T, Ju J, Eyler DE, et al. (2010). Inhibition of eukaryotic translation elongation by cycloheximide and lactimidomycin. Nature Chemical Biology 6(3): Mar. 2010. pp. 209217. DOI: $10.1038 /$ nchembio.304

Schreiber SL (2000). Target-oriented and diversity-oriented organic synthesis in drug discovery. Science 287(5460): Mar. 2000. pp. 1964-1969. DOI: 10.1126/science.287.5460.1964

Schreiber SL (2011). Organic synthesis toward small-molecule probes and drugs. Proc. Natl. Acad. Sci. USA 108(17): Apr. 2011. pp. 6699-6702. DOI: 10.1073/pnas.1103205108

Schuster SC (2008). Next-generation sequencing transforms today's biology. Nature Methods 5(1): Jan. 2008. pp. 16-18. DOI: 10.1038/NMETH1156

Schwendener RA \& Schott H (2010). Liposome Formulations of Hydrophobic Drugs. Liposomes: Methods and Protocols 1(605): pp. 129-138 DOI: 10.1007/978-1-60327-360-2_8

Sen S \& Ferdig M (2004). QTL analysis for discovery of genes involved in drug responses. Current Drug Targets - Infectious Disorders 4(1): Mar. 2004. pp. 53-63. DOI: $10.2174 / 1568005043480916$

Shapiro GI \& Harper JW (1999). Anticancer drug targets: cell cycle and checkpoint control. Journal f Clinical Investigation 104(12): Dec. 1999. pp. 1645-165. DOI: 10.1172/JCI9054

Shendure J \& Hanlee J (2008). Next-generation DNA sequencing. Nature Biotechnology 26(10): Oct. 2008. pp. $1135-1145$. DOI: $10.1038 /$ nbt1486

Shendure J, Porreca GJ, Reppas NB, et al. (2005). Accurate multiplex polony sequencing of an evolved bacterial genome. Science 309(5741): Sep. 2005. pp. 1728-1732. DOI: 10.1126/science.1117389

Shires SE, Kelleher J \& Trejdosiewicz LK (1989). Effects of linoleic-acid and mitogenic stimulation on the fatty-acid composition of human-lymphocytes. Biochimica et Biophysica Acta 1002(1): Mar. 1989. pp. 74-78. DOI: 10.1016/0005-2760(89)90066-0

Short MK, Hallett JP, Tar K, et al. (2012). The yeast magmas ortholog Pam16 has an essential function in fermentative growth that involves sphingolipid metabolism. PLOS One 7(7): Jul. 2012. Art. e39428. DOI: 10.1371/journal.pone.0039428

Silverman WMD (2002). The schizophrenic career of a "monster drug". Pediatrics 110(2): Aug.2002. pp. 404-406. DOI:10.1542/peds.110.2.404.

Simon JA, Szankasi P, Nguyen DK, et al. (2000). Differential toxicities of anticancer agents among DNA repair and checkpoint mutants of Saccharomyces cerevisiae. Cancer Research 60(2): Jan. 2000. pp. 328-333. PMID: 10667584

Singh-Babak SD, Shekhar T, Smith AM, et al. (2012). A novel calcineurin-independent activity of cyclosporin A in Saccharomyces cerevisiae. Molecular BioSystems 8(10): Aug 2012. pp. 2575-84. PMID: 22751784 
Skrtic M, Sriskanthadevan S, Jhas B, et al. (2011). Inhibition of mitochondrial translation as a therapeutic strategy for human acute myeloid leukemia. Cancer Cell 20(5): Nov. 2011. pp. 674-688. DOI: 10.1016/j.ccr.2011.10.015

Sliwkowski MX, Lofgren JA, Lewis GD, et al. (1999). Nonclinical studies addressing the mechanism of action of trastuzumab (Herceptin). Seminars in Oncology 26(4 Suppl. 12): pp. 60-70. PMID: 10482195

Smith AM, Heisler LE, Mellor J, et al. (2009). Quantitative phenotyping via deep barcode sequencing. Genome Research 19(10): Oct. 2009. pp. 1836-1842. DOI: 10.1101/gr.093955.109

Smith V, Botstein D \& Brown PO (1995). Genetic footprinting: A genomic strategy for determining a gene's function given its sequence. Proc. Natl. Acad. Sci. USA 92(14): Jul. 1995. pp. 6479-6483. DOI: 10.1073/pnas.92.14.6479

Sohn LL, Saleh OA, Facer GR, et al. (2000). Capacitance cytometry: Measuring biological cells one-by-one. Proc. Natl. Acad. Sci. USA 97(20): Sep. 2000. pp. 10687-10690. DOI: 10.1073/pnas.200361297

Sohn, JH; Choi, ES; Kang, HA; et al. (1999). A dominant selection system designed for copynumber-controlled gene integration in Hansenula polymorpha DL-1. Applied Microbiology and Biotechnology 51(6): Jun. 1999. pp. 800-807. PMID: 10422227

Stefanidakis G \& Gwyn JE (1993). Alkylation. In Chemical Processing Handbook. McKetta JJ (ed). pp. 80-138. CRC Press. Newark, NJ. ISBN 0-8247-8701-3.

Stephens TD, Bunde CJ \& Fillmore BJ (2000). Mechanism of action in thalidomide teratogenesis. Biochemical Pharmacology 59(12): Jun. 2000. pp. 1489-99. DOI: 10.1016/S00062952(99)00388-3.

Stern M, Jensen R \& Herskowitz I (1984). 5 SWI genes are required for expression of the HO gene in yeast. Journal of Molecular Biology 178(4): pp. 853-868. DOI: 10.1016/00222836(84)90315-2

Stotzky G \& Rem LT (1967). Influence of clay minerals on microorganisms IV: Montmorillonite and kaolinite on fungi. Canadian Journal of Microbiology. 13(11): pp. 1535-\&. PMID: 6070669

Strausberg RL \& Schreiber SL (2003). From knowing to controlling: a path from genomics to drugs using small molecule probes. Science 300(5617): Apr. 2003. pp. 294-295. DOI:10.1126/science.1083395

Szallasi A, Biro T, Modarres S, et al. (1998). Dialdehyde sesquiterpenes and other terpenoids as vanilloids. European Journal of Pharmacology 356(1): Aug. 1998. pp. 81-89. DOI: 10.1016/S0014-2999(98)00514-7

Takano S, Iwabuchi Y \& Ogasawara K (1991). Inversion of enantioselectivity in the kinetic resolution mode of the Katsuki-Sharpless asymmetric epoxidation reaction. Journal of the American Chemical Society 113(7): Mar. 1991. pp. 2786-2787. DOI: 10.1021/ja00007a082

Talbot GH, Bradley J, Edwards JE, et al. (2006). Bad bugs need drugs: an update on the development pipeline from the Antimicrobial Availability Task Force of the Infectious 
Diseases Society of America. Clinical Infectious Diseases 42(7): Apr. 2006. pp. 1065-1065. DOI: $10.1086 / 499819$

Tanida I, Takita Y, Hasegawa A, et al. (1996). Yeast Cls2p/Csg2p localized on the endoplasmic reticulum membrane regulates a non-exchangeable intracellular $\mathrm{Ca} 2+$ pool cooperatively with calcineurin. FEBS Letters 379(1): Jan. 1996. pp. 38-42. DOI: 10.1016/00145793(95)01478-0

Terstappen GC, Schluepen C, Raggiaschi R, et al. (2007). Target deconvolution strategies in drug discovery. Nature Reviews Drug Discovery 6(11): Nov. 2007. pp. 891-903. DOI: $10.1038 / \mathrm{nrd} 2410$

Theil FP, Guentert TW, Haddad S \& Poulin P (2003). Utility of physiologically based pharmacokinetic models to drug development and rational drug discovery candidate selection. Toxicology Letters 138(1-2): Feb. 2003. pp. 29-49. ISSN 0378-4274, 10.1016/S0378-4274(02)00374-0.

Thomas JH, Neff NF \& Botstein D (1985). Isolation and characterization of mutations in the betatubulin gene of Saccharomyces-cerevisiae. Genetics 111(4): pp. 715-734. PMID: 2998923

Thompson AH (2000). Introduction to clinical pharmacokinetics. Paediatric and Perinatal Drug Therapy 4(1): pp. 3-11.

Thomson AJ (2007). The discovery, use and impact of platinum salts as chemotherapy agent for cancer. In Wellcome Trust Witnesses to Twentieth Century Medicine 30. Christie DA \& Tansey EM (eds). pp. 6-15. Wellcome Trust. London, UK. ISBN 978-0-85484-112-7.

Tomasz M (1995). Mitomycin-C - small, fast and deadly (but very selective). Chemistry \& Biology 2(9): Sep. 1995. pp. 575-579. DOI: 10.1016/1074-5521(95)90120-5

Tomasz M, Chawla AK \& Lipman R (1988). Mechanism of monofunctional and bifunctional alkylation of DNA by mitomycin-C. Biochemistry 27(9): May 1988. pp. 3182-3187. DOI: 10.1021/bi00409a009

Tong AHY \& Boone C (2006). Synthetic genetic array analysis in Saccharomyces cerevisiae. In Yeast Protocols $2^{\text {nd }}$ ed: Methods in molecular biology 313. Xiao W (ed). pp. 171-192. Humana Press. Clifton, NJ. ISBN 1592599583

Tong AHY \& Boone C (2007). High throughput strain construction. In Yeast Gene Analysis $2^{\text {nd }}$ ed: Methods in Microbiology 36. Stansfield I \& Starks M (eds). pp. 369-386. Elsevier. London, UK. ISBN-10: 0123694795

Tong AHY, Evangelista, Parsons AB, et al. (2001). Systematic genetic analysis with ordered arrays of yeast deletion mutants. Science 294(5550): Dec. 2001. pp. 2364-2368. DOI: 10.1126/science. 1065810

Tong AHY, Lesage G, Bader GD, et al. (2004). Global mapping of the yeast genetic interaction network. Science 303(5659): Feb. 2004. pp. 808-813. DOI: 10.1126/science.1091317

Tran JR, Tomsic LR \& Brodsky JL (2011). A Cdc48p-associated factor modulates endoplasmic reticulum-associated degradation, cell stress, and ubiquitinated protein homeostasis. J. Biol. Chem. 286(7): Feb. 2011. pp. 5744-5755. DOI: 10.1074/jbc.M110.179259 
Tsai HF, Sammons LR, Zhang X, et al. (2010). Microarray and molecular analyses of the azole resistance mechanism in Candida glabrata oropharyngeal isolates. Antimicrobial Agents and Chemotherapy 54(8): Aug. 2010. pp. 3308-3317. DOI: 10.1128/AAC.00535-10

Tsuchiya D, Gonzalez C \& Lacefield S (2011). The spindle checkpoint protein Mad2 regulates $A P C / C$ activity during prometaphase and metaphase of meiosis I in Saccharomyces cerevisiae. Molecular Biology of the Cell 22(16): Aug. 2011. pp. 2848-2861. DOI: 10.1091/mbc.E11-04-0378

Ueda K, Clark DP, Chen CJ, et al. (1987). The human multidrug resistance (mdr1) gene. cDNA cloning and transcription initiation. J. Biol. Chem. 262(2): Jan. 1987. pp. 505-508. PMID 3027054.

Uetrecht J (2001). Prediction of a new drug's potential to cause idiosyncratic reactions. Current opinion in drug discovery \& development 4(1): Jan.2001. pp. 55-59. PMID: 11727323

Uetrecht J (2008). Idiosyncratic drug reactions: Past, present, and future. Chemical Research in Toxicology 21(1): Jan. 2008. pp. 84-92. DOI: 10.1021/tx700186p

Valeriote F \& Vanputten L (1975). Proliferation-dependent cytotoxicity of anticancer agents - A review. Cancer Research 35(10): pp. 2619-2630

Van Berkum NL \& Holstege FCP (2001). DNA microarrays: raising the profile. Current Opinion in Biotechnology 12(1): Feb. 2001. pp. 48-52. DOI: 10.1016/S0958-1669(00)00173-7

Van Driessche B, Tafforeau L, Hentges $\mathrm{P}$, et al. (2005). Additional vectors for PCR-based gene tagging in Saccharomyces cerevisiae and Schizosaccharomyces pombe using nourseothricin resistance. Yeast 22(13): Oct. 2005. pp. 1061-1068. DOI: 10.1002/yea.1293

Vandeputte P, Ferrari S \& Coste AT (2011). Antifungal resistance and new strategies to control fungal infections. International Journal of Microbiology 2012. pp. 713687-\&. DOI: $10.1155 / 2012 / 713687$

Varki A (2009). Essentials of Glycobiology $2^{\text {nd }}$ ed. Varki A (ed.). Cold Spring Harbor Laboratories Press. Cold Spring Harbor, NY. ISBN 978-0-87969-770-9.

Vedula SS, Bero L, Scherer RW \& Dickersin K (2009). Outcome reporting in industry-sponsored trials of gabapentin for off-label use. New England Journal of Medicine 361(20): Nov. 2009. pp. 1963-71. DOI:10.1056/NEJMsa0906126.

Verheij H \& Robeson BL (2002). The role of chemical diversity in drug discovery. Genome Proteome Technol. Sep. 2002. pp 32-35. Retrieved 2-7-2012 from http://www.spectrumbio.com/docs/Genomic Proteomic.pdf

Von Nussbaum F, Brands M, Hinzen B, et al. (2006). Antibacterial natural products in medicinal chemistry--exodus or revival?. Angewandte Chemie-International Edition 45 (31): pp. 5072-129. DOI: 10.1002/anie.200600350.

Wang $\mathrm{H}$, Zhang $\mathrm{C}$, Rorick A, et al. (2011). CCI-779 inhibits cell-cycle $\mathrm{G}_{2}-\mathrm{M}$ progression and invasion of castration-resistant prostate cancer via attenuation of UBE2C transcription and mRNA stability. Cancer Research 71(14): Jul. 2011. pp. 4866-4876. DOI: 10.1158/00085472.CAN-10-4576 
Wang JL, Zhang ZJ, Choksi S, et al. (2000). Cell permeable Bcl-2 binding peptides: A chemical approach to apoptosis induction in tumor cells. Cancer Research 60(6): Mar. 2000. pp. 1498-1502. PMID: 10749111

Warrington B (2003). High throughput drug discovery. GlaxoSmithKline R\&D. Harlow, Essex, UK.

Wartlick H, Michaelis K, Balthasar S, et al. (2004). Highly specific HER2-mediated cellular uptake of antibody-modified nanoparticles in tumour cells. Journal of Drug Targeting 12(7): pp. 461-471. DOI: 10.1080/10611860400010697

Warwick GP (1963). Mechanism of action of alkylating agents. Cancer Research 23(8): pp. 1315\&. PMID: 14070386

Weisblum B (1995). Erythromycin resistance by ribosome modification. Antimicrobial Agents and Chemotherapy 39(3): Mar. 1995. pp. 577-585. PMID: 7793855

Wendler F, Gillingham AK, Sinka R, et al. (2010). A genome-wide RNA interference screen identifies two novel components of the metazoan secretory pathway. EMBO Journal 29(2): Jan. 2010. pp. 304-314. DOI: 10.1038/emboj.2009.350

Winston $\mathrm{F}$ (2008). EMS and UV mutagenesis in yeast. In Current protocols in molecular biology. Ausubel FM (ed). pp. 13.3B\&. John Wiley \& Sons. New York, NY. ISBN-10: 047150338X

Winzeler EA, Shoemaker DD, Astramoff A, et al. (1999). Functional characterization of the Saccharomyces cerevisiae genome by precise deletion and parallel analysis. Science 285(5429): Aug. 1999. pp. 901-906. DOI: 10.1126/science.285.5429.901

Xiao W, Derfler B, Chen J, et al. (1991). Primary sequence and biological functions of a Saccharomyces-cerevisiae O6-methylguanine O4-methylthymine DNA-repair methyltransferase gene. EMBO Journal 10(8): Aug. 1991. pp. 2179-2186.

Xie MW, Jin F, Hwang $\mathrm{H}$, et al. (2005). Insights into TOR function and rapamycin response: Chemical genomic profiling by using a high-density cell array method. Proc. Natl. Acad. Sci. USA 102(20): May 2005. pp. 7215-7220. DOI: 10.1073/pnas.0500297102

Xu H, Boulianne GL \& Trimble WS (2002). Membrane trafficking in cytokinesis. Seminars in Cell \& Developmental Biology 13(2): Apr. 2002. pp. 77-82. DOI: 10.1016/S1084-9521(02)000125

Yadav J, Muend S, Zhang Y, et al. (2007). A phenomics approach in yeast links proton and calcium pump function in the Golgi. Molecular Biology of the Cell 18(4): Apr. 2007. pp. 1480-1489. DOI: 10.1091/mbc.E06-11-1049

Yan J \& Burgess SM (2012). Using a yeast inverse one-hybrid system to identify functional binding sites of transcription factors. In Gene Regulatory Networks: Methods and Protocols. Deplancke B \& Gheldof N (eds). pp. 275-290. Humana Press. Clifton, NJ. ISBN-10: 1617792918. DOI: 10.1007/978-1-61779-292-2_17

Yang H, Ganguly A \& Cabral F (2010). Inhibition of cell migration and cell division correlates with distinct effects of microtubule inhibiting drugs. J. Biol. Chem. 285(42): Oct. 2010. pp. 32242-32250. DOI: 10.1074/jbc.M110.160820 
Yang Z \& Sankoff D (2011). Generalized adjacency and the conservation of gene clusters in genetic networks defined by synthetic lethality. IEEE International Conference on Bioinformatics and Biomedicine 13(9): Nov. 2011. Art. S8. DOI: 10.1186/1471-2105-13-S9S8

Yibmantasiri P, Leahy DC, Busby BP, et al. (2012) Molecular basis for fungicidal action of neothyonidioside, a triterpene glycoside from the sea cucumber, Australostichopus mollis. Molecular Biosystems 8(3): pp. 902-912. DOI: 10.1039/c2mb05426d

Zachara NE, O'Donnell N, Cheung WD, et al. (2004). Dynamic O-GlcNAc modification of nucleocytoplasmic proteins in response to stress. J Biol Chem 279(29): Jul. 2004. pp. 30133-30142. DOI: 10.1074/jbc.M403773200

Zaman S, Lippman SI, Zhao X, et al. (2008). How Saccharomyces responds to nutrients. Annual Review of Genetics 42: pp. 27-81. DOI: 10.1146/annurev.genet.41.110306.130206

Zhanel GG, Karlowsky JA, Zelenitsky SA, et al. (1998). Susceptibilities of Candida species isolated from the lower gastrointestinal tracts of high-risk patients to the new semisynthetic echinocandin LY303366 and other antifungal agents. Antimicrobial Agents and Chemotherapy 42(9): Sep. 1998. pp. 2446-2448. PMID: 9736582

Zhao R, Davey M, Hsu YC, et al. (2005). Navigating the chaperone network: an integrative map of physical, genetic, and chemical-genetic interactions mediated by the yeast Hsp90 chaperone system. Cell 120(5): Mar. 2005. pp. 715-727. DOI: 10.1016/j.cell.2004.12.024

ZoBell CE (1943). The effect of solid surfaces upon bacterial activity. Journal of Bacteriology 46(1): Jul. 1943. pp. 39-56. PMID: 1656067 
